LUCAS HENRIQUE SOUZA DO CARMO

\title{
A slender-body approach for computing second-order wave forces in seakeeping simulations of floating offshore wind turbines
}

São Paulo 



\section{A slender-body approach for computing second-order wave forces in seakeeping simulations of floating offshore wind turbines}

\section{Revised Version}

Thesis submitted to the Escola Politécnica da Universidade de São Paulo for the partial fulfillment of the requirements for the degree of Doctor of Science

Concentration Area: Naval Architecture and Ocean Engineering

Supervisor: Prof. Alexandre Nicolaos Simos

São Paulo 
Autorizo a reprodução e divulgação total ou parcial deste trabalho, por qualquer meio convencional ou eletrônico, para fins de estudo e pesquisa, desde que citada a fonte.

Este exemplar foi revisado e corrigido em relação à versão original, sob responsabilidade única do autor e com a anuência de seu orientador.

São Paulo, de de

Assinatura do autor:

Assinatura do orientador:

\section{Catalogação-na-publicação}

Carmo, Lucas Henrique Souza do

A slender-body approach for computing second-order wave forces in seakeeping simulations of floating offshore wind turbines / L. H. S. Carmo -versão corr. -- São Paulo, 2021.

$209 \mathrm{p}$.

Tese (Doutorado) - Escola Politécnica da Universidade de São Paulo. Departamento de Engenharia Naval e Oceânica.

1.Turbinas eólicas flutuantes 2 .Hidrodinâmica 3.Forças de onda de segunda ordem 4.Aproximação de corpo esbelto I.Universidade de São Paulo. Escola Politécnica. Departamento de Engenharia Naval e Oceânica II.t. 


\section{Acknowledgements}

The past four years or so have been a great experience. Frequently exhausting and not always pleasing, but extremely rewarding nonetheless. I would like to thank some people that have played an important role in this journey.

First of all, I am really thankful to my academic mentor, Prof. Alexandre Simos, for supervising my research since my undergraduate studies. He has always let me free to do my work, but at the same time following it closely with precious advice, which have been fundamental to this thesis. He is an inspiration not only as an engineer and researcher, but also as a person. It was a pleasure to work under his guidance, and I hope this partnership will continue for many years to come.

I would also like to thank the members of my thesis committee, Prof. Antonio Souto-Iglesias, Prof. Paulo de Tarso Themistocles Esperança, Prof. Celso Pupo Pesce and Prof. José Augusto Penteado Aranha, for their time and the valuable suggestions and corrections that improved this work significantly. More than that, Prof. Aranha and Prof. Pesce, just like Prof. Simos, have shaped my way of viewing engineering and science since my early years as an undergraduate student, and I am still amazed by classes that I had with them many years ago. When things got too complicated, I often found comfort thinking that they were students once too and faced their own struggles to be who they are nowadays, and this thought assured me of my decision to be a researcher. And this might sound crazy, but I confess that I frequently have imaginary chats with the three of them to question my ideas and hypothesis.

I am indebted to Giovanni Amaral and Pedro de Mello for conducting most of the experiments presented in this text, which helped me avoid taking the subway during the height of the pandemic. Giovanni has also helped me immensely in using his formulation to compute the stiffness matrix due to the moorings. I am also thankful to Edgard Malta and Raul Dotta for developing Edtools, which was extremely useful to prepare the experiments.

Thank you to the "elders" of the Numerical Offshore Tank (TPN-USP) for sharing their knowledge. Edgard Malta, Daniel Vieira, Rafael Watai, Felipe Ruggeri and Jordi Soler (ok, this one is not that old) have all had a share in teaching me how to use WAMIT, besides the interesting discussions on several topics.

Thank you very much to my dear friend, Prof. Rodolfo Gonçalves, and Prof. Hideyuki Suzuki, Prof. Toru Sato, Ms. Chieko Doi and Prof. Shinichiro Hirabayashi for the very fruitful period I spent at the National Maritime Research Institute of Japan and at the University of Tokyo. It was an amazing experience, both professionally and personally. The trip to the Fukushima floating offshore wind demonstration site, which was the first 
time I saw a floating wind turbine, is perhaps my favorite of the many special moments I experienced in Japan. Thank you also to the students of the Ocean Space Planning Laboratory for such a warm welcome. And I really miss the sushi.

A special thanks to my academic siblings, Lariuss Zago and Fernanda Hille, and to all my friends from TPN, Giovanni Amaral, Kam Kang, Raiza da Silva, Cristiana Pirpiris, Jordi Soler, among others, for the laughs, coffees, discussions, meals, etc. You have made this period far more enjoyable, and I wish you all the best!

I thank my parents, Rosângela Souza and Sérgio do Carmo, and sisters, Lara and Paula, for all their love and support. My family has been my first funding agency, providing microscopes, chemistry sets and all sorts of equipment to my small laboratory, besides all the books and science magazines that I wanted. My father would always take me to the construction sites that he was working on, and it was awesome to watch so closely the buildings being built, while my mother would bring me lab glassware very frequently.

I really think that I would not have started or finished my $\mathrm{PhD}$ without the support of my wife, Letícia Tomazoli, and she deserves a very special thanks (or should I blame her?). We have been through so much together, and I am really glad that she was by my side and helped me so much in achieving this dream of mine. She never doubted any of the times that I claimed that this thesis would be finished by the end of the month (for about half a year), and deserves much of the credit for this work. Our dogs, Bibi and Leia, have also been a constant company and reminded me that sometimes it is worth taking a break to play fetch. I love you dearly.

Finally, I would like to thank the University of São Paulo, embodied by its professors, staff and students, for giving me purpose by teaching me the importance of science to the development of our country and humankind in general. It is not easy to do research in Brazil, specially with the recent wave of anti-intellectualism, but I am really proud to be part of this community. Scientia vinces.

This study was financed in part by the Coordenação de Aperfeiçoamento de Pessoal de Nível Superior - Brasil (CAPES) - Finance Code 001. 
"O senhor... mire, veja: o mais importante e bonito, do mundo, é isto: que as pessoas não estão sempre iguais, ainda não foram terminadas - mas que elas vão sempre mudando. Afinam ou desafinam, verdade maior. É o que a vida me ensinou. Isso que me alegra montão." João Guimarães Rosa, Grande Sertão: Veredas 



\section{Abstract}

This thesis describes the development of METiS, a numerical tool for the seakeeping analysis of floating offshore wind turbines (FOWT). It is based on a slender-body approximation for evaluating the first- and second-order wave loads acting on a floating structure comprised of slender cylinders that combines Rainey's equation, which can be seen as an extension of the inertial part of Morison's equation to include nonlinear terms, with Pinkster's formulation for the low-frequency second-order loads on floating bodies. This combination is followed to allow the evaluation of the forces considering the mean body position, in such a way that an Inverse Fast Fourier Transform algorithm can be used to efficiently compute the time series of wave kinematics and second-order wave loads in a real sea condition directly in time domain, as opposed to the most common procedure of solving the second-order radiation/diffraction problem in frequency domain and importing the results to time-domain solvers. As a drawback of the approximation, end effects due to the extremities of the cylinder and effects due to wave scattering and radiation are lost, which is acceptable as long as the diameters of the cylinders that compose the structure are small in face of their draft and in face of the length of the incoming waves. These conditions may be too restrictive to modern oil \& gas spars and semi-submersibles, which have large diameter columns, but it is satisfied by FOWTs in many significant wave conditions.

The slender-body approximation is first verified by analyzing the simple case of a single surface piercing cylinder under the action of bichromatic waves, both bottom mounted and floating, for different combinations of the dimensionless parameters that describe the problem. The results are compared with the ones obtained with diffraction theory and Newman's approximation, evidencing an interesting complementarity between the two approximations and the conditions in which each of them performs the better. The relevance of the second-order terms from Rainey's formulation is shown, demonstrating that the common practice of analyzing second-order loads by simply applying Morison's equation with second-order wave kinematics is not strictly correct.

The method is then applied to the analysis of a semi-submersible FOWT model, moored by three caternary lines, that was tested at the wave basin of the Numerical Offshore Tank of the University of São Paulo. Three sets of tests are presented: free decays of the moored model; forced oscillations of the hull; and motions under the action of waves (bichromatic, JONSWAP and white-noise) and wind. The results obtained with METiS are compared with the experiments and with WAMIT and OpenFAST, illustrating the capabilities and limitations of each software.

Keywords: Floating offshore wind turbines. Hydrodynamics. Second-order wave loads. Slender body approximation. 



\section{Resumo}

Esta tese descreve o desenvolvimento de uma ferramenta numérica, denominada METiS, para a análise do comportamento no mar de turbinas eólicas flutuantes (FOWTs). Ela é baseada em uma aproximação de corpo esbelto para calcular as forças de onda de primeira e segunda ordem em estruturas compostas de cilindros esbeltos que combina a equação de Rainey, que pode ser vista como uma extensão da equação de Morison para incluir termos inerciais não lineares, com a formulação de Pinkster para as forças de segunda ordem em corpos flutuantes. Esta combinação é adotada para permitir a determinação das forças considerando a posição média do corpo, de tal forma que uma Transformada Rápida de Fourier pode ser usada para calcular de forma eficiente as séries temporais de cinemática das ondas e das forças de segunda ordem para um mar real diretamente no domínio do tempo, diferentemente do procedimento usual de resolver o problema de radiação/difração de segunda ordem no domínio da frequência e importar os resultados para códigos no domínio do tempo. Como desvantagem, efeitos de ponta devido às extremidades do cilindro e efeitos de radiação e espalhamento de ondas são perdidos, o que é aceitável desde que os diâmetros dos cilindros que compõem a estrutura sejam pequenos frente ao calado e ao comprimento das ondas. Essas condições podem ser restritivas para plataformas modernas do tipo spar e semissubmersível, que têm colunas de grande diâmetro, mas são satisfeitas por FOWTs em muitas condições de ondas relevantes.

A aproximação de corpo esbelto é primeiro verificada para o caso simples de um único cilindro cruzando a superfície livre, tanto fixo quanto flutuante, sob a ação de ondas bicromáticas para diferentes combinações dos adimensionais que descrevem o problema. Os resultados são comparados com os obtidos com a teoria de difração de ondas e a aproximação de Newman, evidenciando uma interessante complementaridade entre as duas aproximações e as condições em que cada uma delas é mais apropriada. Mostra-se a relevância dos termos de segunda ordem da formulação de Rainey, demonstrando-se que a prática usual de calcular forças de segunda ordem pela simples aplicação da equação de Morison com cinemática de ondas de segunda ordem não é estritamente correta.

O método é então aplicado à análise de um modelo de uma FOWT do tipo semissubmersível, ancorada por três linhas em catenária, que foi ensaiado no tanque de provas do Tanque de Provas Numérico da Universidade de São Paulo. São apresentados três conjuntos de testes: decaimento livre do modelo ancorado; oscilações forçadas do casco; e movimentos sob a ação de ondas (bicromáticas, JONSWAP e ruído branco) e vento. Os resultados obtidos com o METiS são comparados com os experimentais e com os calculados com os software WAMIT e OpenFAST, ilustrando as capacidades e limitações de cada ferramenta.

Palavras-chave: Turbinas eólicas flutuantes. Hidrodinâmica. Forças de onda de segunda ordem. Aproximação de corpo esbelto. 



\section{List of Figures}

Figure 1 - Global cumulative installed wind capacity from 2001 to 2020, including both onshore and offshore capacity. . . . . . . . . . . . . . . 23

Figure 2 - Some concepts of offshore wind turbines. The first three on the left are bottom-fixed, while the three on the right are the most common floating concepts, namely a semi-submersible, a tension-leg platform (TLP), and a spar. . . . . . . . . . . . . . . . . . 25

Figure 3 - WindFloat in dry dock (left) and being towed to the place of installation (right) . . . . . . . . . . . . . . . . .

Figure 4 - Power spectral density of the tower-base shear force for an operational wave excitation (JONSWAP with significant height equal to $7.1 \mathrm{~m}$ and peak period of $12.1 \mathrm{~s}$, without wind nor sea current) calculated during the OC5 project by the different participants. . . . . . . . . .

Figure 5 - Superposition of the diffraction problem (incoming waves disturbed by the structure, considered to be fixed) with the radiation problem (waves generated by body motions, considering the sea to be perfectly calm). . 36

Figure 6 - Systems of coordinates. . . . . . . . . . . . . . 46

Figure 7 - Segment of length $\mathrm{d} z$ of the cylinder, illustrating the global and local coordinate systems. . . . . . . . . . . . . . . . . . 5 54

Figure 8 - Flowchart with the main steps of the numerical method. . . . . . . . 80

Figure 9 - Illustration of the bottom mounted vertical cylinder under the action of a bichromatic wave. . . . . . . . . . . . . . . . . 82

Figure 10 - Nondimensional force and moment QTFs calculated with diffraction theory for $\bar{\omega}_{j l}^{-}=0.300$ and different values of $\bar{h} . \ldots \ldots$. . . . .

Figure 11 - Nondimensional force and moment QTFs calculated with diffraction theory for $\bar{h}=15$ and different values of $\bar{\omega}_{j l}^{-} \ldots \ldots . \ldots 88$

Figure 12 - Nondimensional force per unit length along the cylinder for $\bar{h}=10$, $\bar{\omega}_{j l}^{-}=0.300$ and $\bar{k}_{j}=\pi / 10$ (i.e. $\lambda_{j} / D=10$ ). . . . . . . . . . .

Figure 13 - Nondimensional horizontal force acting on the bottom mounted cylinder calculated with diffraction theory, the slender-body and Newman's approximations for $\bar{\omega}_{j l}^{-}=0.300$ and $\bar{h}=20 \ldots \ldots$. . . . . . 94

Figure 14 - Components of the nondimensional horizontal force acting on the bottom mounted cylinder calculated with diffraction theory and the slender-body approximation for $\bar{\omega}_{j l}^{-}=0.300$ and $\bar{h}=20 \ldots \ldots$. . . . . . . 96

Figure 15 - Components of the nondimensional horizontal force acting on the bottom mounted cylinder calculated with diffraction theory and the slender-body approximation for $\bar{\omega}_{j l}^{-}=0.300$ and $\lambda_{j} / D=10 \ldots \ldots . \ldots 97$ 
Figure 16 - Components of the nondimensional horizontal force acting on the bottom mounted cylinder calculated with diffraction theory and the slender-body approximation for $\bar{h}=20$ and $\lambda_{j} / D=10 \ldots \ldots$. . . . . . 98

Figure 17 - Nondimensional moment, with respect to the mean water line, acting on the bottom mounted cylinder calculated with diffraction theory, the slender-body and Newman's approximations for $\bar{\omega}_{j l}^{-}=0.300$ and $\bar{h}=20.99$

Figure 18 - Higher-order mesh of the cylinder with $\bar{d}=5.3 . \ldots .$. . . . . . 103

Figure 19 - Mean surge force acting on the cylinder with $\bar{d}=5.3$ for different panel sizes. . . . . . . . . . . . . . . . . . 103

Figure 20 - Added mass coefficients needed to match the added mass terms computed with WAMIT for the long (left) and short (right) cylinders. . . . 105

Figure 21 - Horizontal force due to the convective acceleration calculated with METiS for the short cylinder under the action of the BIC01 wave and $\bar{\omega}_{j l}=0.555$ for different discretizations. . . . . . . . . . . 106

Figure 22 - Surge motion of the short cylinder for the BIC01 wave and $\bar{\omega}_{j l}=0.555$ for different time steps. . . . . . . . . . . . . . . . . . . 107

Figure 23 - First-order nondimensional surge (top), heave (middle) and pitch (bottom) loads for the long (left) and short (right) cylinders. . . . . . . . . 109

Figure 24 - Surge (top), heave (middle) and pitch (bottom) RAOs for the long (left) and short (right) cylinders. . . . . . . . . . . . . . . . . . 110

Figure 25 - Amplitude of the nondimensional second-order horizontal force acting on the long (left $\left.-\bar{\omega}_{j l}^{-}=0.300\right)$ and short (right $\left.-\bar{\omega}_{j l}^{-}=0.259\right)$ cylinders calculated with and without first-order motions as a function of the length of the shortest wave of the pair. . . . . . . . . . . . . . . . 111

Figure 26 - Real and imaginary parts of the nondimensional second-order horizontal force acting on the long (left $\left.-\bar{\omega}_{j l}^{-}=0.300\right)$ and short (right $\left.-\bar{\omega}_{j l}^{-}=0.259\right)$ cylinders as a function of the length of the shortest wave of the pair. . 111

Figure 27 - Amplitude of the components of the nondimensional second-order horizontal force acting on the long (left $\left.-\bar{\omega}_{j l}^{-}=0.300\right)$ and short (right $\left.\bar{\omega}_{j l}^{-}=0.259\right)$ cylinders calculated with METiS as a function of the length of the shortest wave of the pair. The definition of each component is given in Section 3.6. . . . . . . . . . . . . . . . . . . . 113

Figure 28 - Amplitude of the nondimensional second-order moment, with respect to the mean water line, acting on the long (left $\left.-\bar{\omega}_{j l}^{-}=0.827\right)$ and short (right $\left.-\bar{\omega}_{j l}^{-}=0.555\right)$ cylinders calculated with and without first-order motions as a function of the length of the shortest wave of the pair. . . 114

Figure 29 - Illustration of the JPK floating wind turbine. . . . . . . . . . . . 115

Figure 30 - Pictures of the models analyzed in the first (left) and second (right) phases of the project. . . . . . . . . . . . . 116 
Figure 31 - Wave basin of the Numerical Offshore Tank of the University of São Paulo. . . . . . . . . . . . . . . . . . . 117

Figure 32 - Isometric and top views of the Edtools model showing the position of the ballast weights inside the columns. . . . . . . . . . . . 118

Figure 33 - Isometric and top views of the Edtools model illustrating the mooring lines. The chain segment is in blue, while the one made of polyethylene is in red. . . . . . . . . . . . . . . . . . . . . . . 120

Figure 34 - Pictures of the setup of the horizontal (left) and vertical (right) forced oscillation tests. . . . . . . . . . . . . . . . . . 123

Figure 35 - Added mass and drag coefficients obtained from the forced oscillations. 125

Figure 36 - Higher-order mesh of the JPK floater. . . . . . . . . . . . . . . . . 128

Figure 37 - Mean surge force on the floater for different panel sizes. . . . . . . . . . 129

Figure 38 - Free surface mesh used to check the relevance of the free-surface forcing terms on the QTFs calculated with WAMIT. . . . . . . . . . . . 131

Figure 39 - Surge and pitch QTFs at the difference frequency corresponding to their natural frequencies 1) without the second-order potential, 2) with the second-order potential, but neglecting the free-surface forcing terms and 3) with the complete second-order potential. . . . . . . . . . . . . 131

Figure 40 - Color map of the nondimensional force/moment amplitude QTF matrices (left) and their diagonals around the corresponding natural frequency of motion for surge, heave and pitch (right). The frequency range with wave energy is indicated in blue. . . . . . . . . . . . . . . . . . . . 132

Figure 41 - Higher-order mesh for the simulations with wind (inclination of $-5.1^{\circ}$ around the global Y axis). . . . . . . . . . . . . . . . . . . 133

Figure 42 - Nondimensional force/moment amplitude QTFs (for the most relevant diagonals) obtained with and without wind effects.

Figure 43 - Horizontal drag force calculated with METiS for different discretizations.135

Figure 44 - Comparison between the wave elevation measured in the experiments and the one simulated with METiS for the WHI01 wave condition. . . 136

Figure 45 - Numerical surge decays obtained with OpenFAST for three different time steps. . . . . . . . . . . . . . . . . . . . . . . 138

Figure 46 - Results obtained from the decay tests without wind. . . . . . . . . . . 140

Figure 47 - Results obtained from the decay tests with wind and rotor speeds equal to $7.8 \mathrm{~m} / \mathrm{s}$ and $9.07 \mathrm{rpm}$. Due to the few peaks observed in pitch, the corresponding PQ analysis must be ignored. . . . . . . . . . . . . . . . 141

Figure 48 - PQ analysis of the decoupled decay simulations neglecting drag due to the moorings. The experimental results are the same from the previous graphs. . . . . . . . . . . . . . . . . . . . . . 143 
Figure 49 - Nondimensional force/moment amplitude in surge, heave, pitch and yaw calculated with WAMIT and METiS for a wave incidence of $210^{\circ}$. $\bar{R}=5.25 \mathrm{~m}$ is the mean column radius. . . . . . . . . . . . . 146

Figure 50 - Nondimensional force/moment amplitude in surge, heave and pitch acting on the floater considering its undisplaced position and the mean displacement induced by the wind for a wave incidence of $180^{\circ}$. . . . . 147

Figure 51 - Surge, heave and pitch RAOs (with respect to the CoG) for a wave incidence of $180^{\circ}$. . . . . . . . . . . . . . . . . . . . 149

Figure 52 - Pitch RAO (with respect to the CoG) for an incidence of $180^{\circ}$ accounting for aerodynamic thrust on the rotor. . . . . . . . . . . . . . . . 150

Figure 53 - Heave RAO for a wave incidence of $180^{\circ}$ obtained with the bichromatic waves.

Figure 54 - Nondimensional second-order surge force calculated with WAMIT and METiS. . . . . . . . . . . . . . . . . . . 152

Figure 55 - Difference-frequency second-order surge motion measured in the experiments and calculated with WAMIT and METiS. . . . . . . . . . . . . . 152

Figure 56 - Impact of mooring stiffness $\left(\mathbb{K}_{M, G, 11}\right)$ on the second-order surge motion. 153

Figure 57 - Excerpt of the surge motion measured in the experiments for the JON01 wave. . . . . . . . . . . . . . . . . . . . . . 154

Figure 58 - Wave elevation and motion spectra for the JON02 wave. . . . . . . . . 156

Figure 59 - Wave elevation and motion spectra for the JON03 wave. . . . . . . . . 158

Figure 60 - Wave elevation and motion spectra for the JON04 wave. . . . . . . . . 159

Figure 61 - Wave elevation and motion spectra for the JON04 wave considering adjusted drag coefficients. . . . . . . . . . . . . . . . . . 160

Figure 62 - Wave elevation and motion spectra for the JON01 wave. . . . . . . . . 162

Figure 63 - Wave elevation and motion spectra for the JON02 with and without wind.163

Figure 64 - Spectrum of pitch motion for the JON01 wave with and without wind, zoomed to show the response at the wave frequency. . . . . . . . . . . . 164

Figure 65 - Nondimensional potential damping in surge $\left(B_{11}\right)$ and heave $\left(B_{33}\right)$ calculated with WAMIT. . . . . . . . . . . . . . . 206

Figure 66 - Time series (top) and discrete fourier transform (bottom) of the hydrodynamic force in surge direction measured in the experiments before and after the filtering process for $T=12 \mathrm{~s}$ and $A=4.8 \mathrm{~m}$. The force calculated with Morison's equation considering the resulting $C_{a}$ and $C_{D}$ is also plotted. 
Figure 67 - Time series (top) and discrete fourier transform (bottom) of the hydrodynamic force in surge direction measured in the experiments before and after the filtering process for $T=84.4 \mathrm{~s}$ and $A=0.8 \mathrm{~m}$. The force calculated with Morison's equation considering the resulting $C_{a}$ and $C_{D}$

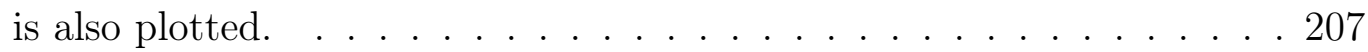





\section{List of Tables}

Table 1 - Selected points from the first graph of Figure 13 to ease the comparisons. 94

Table 2 - Imaginary part of the forces at selected points of Figure 14. . . . . . . . 96

Table 3 - Imaginary part of the forces at selected points of Figure 15 . . . . . . . 97

Table 4 - Imaginary part of the forces at selected points of Figure 16. . . . . . . 98

Table 5 - Properties of the floating cylinders. . . . . . . . . . . 100

Table 6 - List of the bichromatic waves simulated for the long cylinder for each

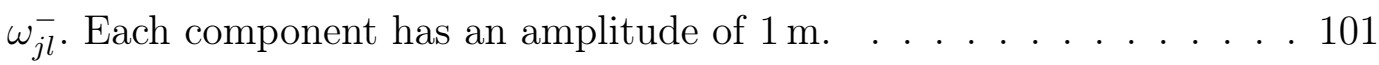

Table 7 - List of the bichromatic waves simulated for the short cylinder for each

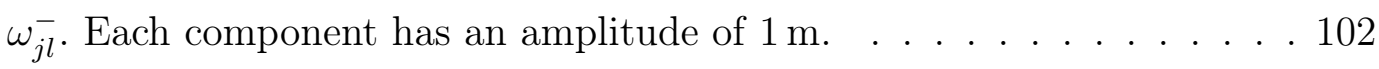

Table 8 - External linear heave and pitch damping considered in the WAMIT simulations. . . . . . . . . . . . . . . . . . . . . . 104

Table 9 - Main properties of the JPK FOWT. Natural periods were obtained from decay tests of the moored model without wind emulation. . . . . . . . 118

Table 10 - Main properties of the RNA . . . . . . . . . . . . . . 119

Table 11 - Main properties of the mooring system. . . . . . . . . . . . . 120

Table 12 - Wind condition considered in the tests. . . . . . . . . . . . . . 121

Table 13 - White-noise wave characteristics. . . . . . . . . . . . . . . . 121

Table 14 - Characteristics of the bichromatic waves $\left(\omega_{1}-\omega_{2}=0.073 \mathrm{rad} / \mathrm{s}\right)$. Each component has a height equal to $1 \mathrm{~m}$. . . . . . . . . . . . . 121

Table 15 - Characteristics of the JONSWAP waves analyzed. They were tested for a duration of $3 \mathrm{~h}$ in full scale $(1207 \mathrm{~s}$ in model scale). . . . . . . . . . . . 121

Table 16 - Motion amplitudes of the forced oscillations and corresponding $K C$ values. 123

Table 17 - Motion periods considered in the forced oscillations and corresponding $\beta$ values. . . . . . . . . . . . . . . . . . . . . . . . 123

Table 18 - Motion amplitudes calculated from the experiments considering bichromatic waves (incidence equal to $180^{\circ}$ ) without wind, with their respective Keulegan-Carpenter numbers and resulting $C_{a}, C_{D}$ and $\zeta_{1}$ obtained from forced oscillations. . . . . . . . . . . . . . . . . . . . . . . 127

Table 19 - Drag and added mass coefficients of the columns of the JPK considered in general conditions, including the WHI and JON waves. . . . . . . . . 127

Table 20 - External linear heave and pitch damping considered in the WAMIT simulations. . . . . . . . . . . . . . . . . . 130

Table 21 - Natural periods from decay tests. . . . . . . . . . . . . . . . . . 139

Table 22 - Mean displacements induced by the wind. . . . . . . . . . . . . . . 142

Table 23 - Ratio between linear $\left(B_{\text {lin }}\right)$ and critical damping obtained from decays. . 143 
Table 24 - Quadratic damping $\left(B_{\text {quad }}\right)$ from decay tests. Units for surge and heave are N.s $\mathrm{s}^{2}$, while for pitch it is N.m.s ${ }^{2} \cdot \operatorname{rad}^{-2}$. . . . . . . . . . . . . 144

Table 25 - Ranges of frequencies considered for computing the amplitudes around the surge and pitch resonances (red shaded in the graphs) and at the frequencies of the incoming waves (blue shaded). . . . . . . . . . . 155

Table 26 - Motion amplitudes calculated with Equation 6.24 for the JON02 wave. . 156

Table 27 - Motion amplitudes calculated with Equation 6.24 for the JON03 wave. . 158

Table 28 - Motion amplitudes calculated with Equation 6.24 for the JON04 wave. . 159

Table 29 - Motion amplitudes calculated with Equation 6.24 for the JON04 wave considering adjusted drag coefficients. . . . . . . . . . . . . . 160

Table 30 - Motion amplitudes calculated with Equation 6.24 for the JON01 wave. . 162

Table 31 - Ratio between motion amplitudes with and without wind $\left(A_{\alpha}^{\text {wind }} / A_{\alpha}\right) . \quad 163$ 


\section{Contents}

1 INTRODUCTION $\ldots \ldots \ldots \ldots \ldots \ldots \ldots \ldots \ldots$

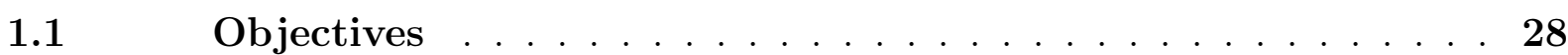

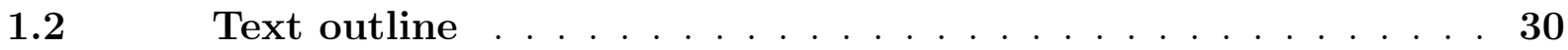

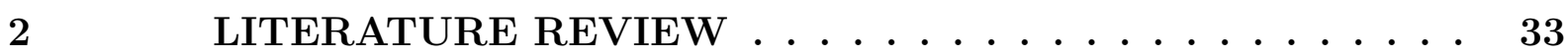

$2.1 \quad$ Main approaches for seakeeping analysis . . . . . . . . 34

$2.2 \quad$ Slender-body approximation for second-order wave loads . . . . 39

3 SLENDER-BODY APPROXIMATION FOR SECOND-ORDER

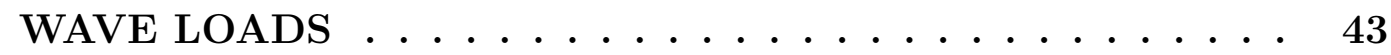

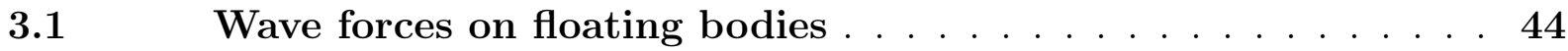

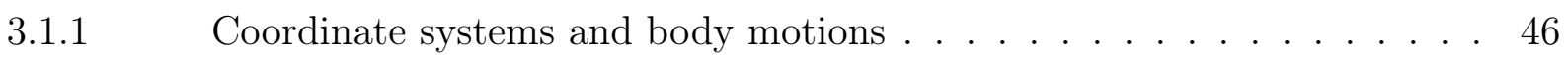

3.1.2 First- and second-order wave forces . . . . . . . . . . . 48

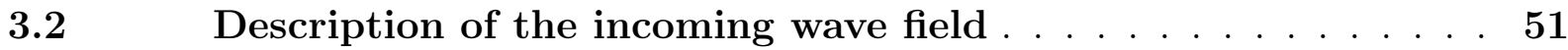

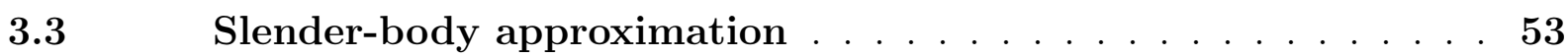

3.3.1 Potential flow round a moving cylinder . . . . . . . . . . . 54

3.3.2 Forces along the length of the cylinder . . . . . . . . . . . . . 56

3.3.3 Forces at the extremities of the cylinder . . . . . . . . . . . . 59

3.3.4 Modifications to evaluate the forces at the mean body position . . . . . 60

$3.4 \quad$ Viscous effects . . . . . . . . . . . . . . . 64

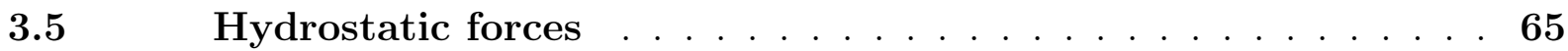

3.6 Total hydrodynamic forces acting on the floater . . . . . . . 67

$3.7 \quad$ The choice of $C_{a}$ and $C_{D} \ldots \ldots \ldots \ldots \ldots \ldots$

4 NUMERICAL METHOD . . . . . . . . . . . . 73

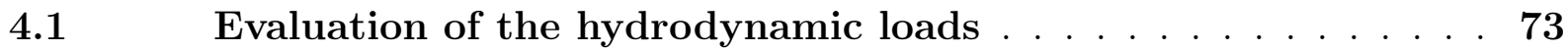

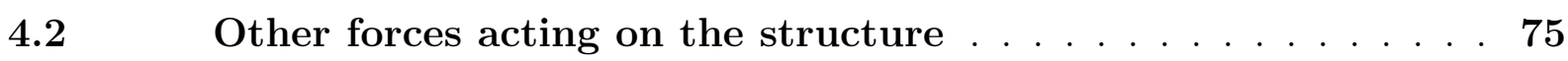

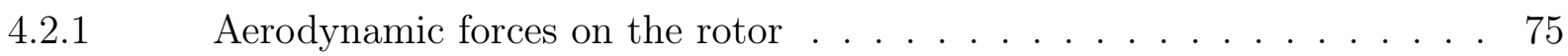

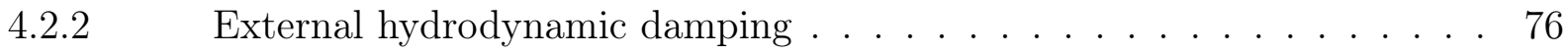

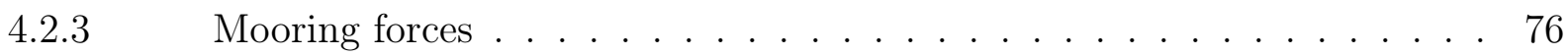

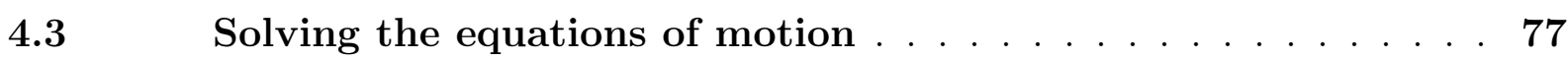

4.4 Outline of the numerical method . . . . . . . . . . . 79

5 LOW-FREQUENCY WAVE FORCES ON A SURFACE-PIERCING

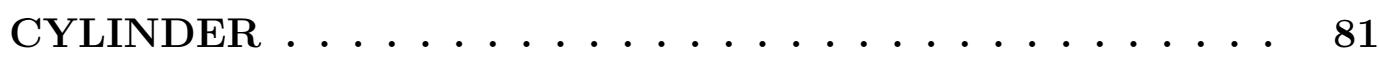

$5.1 \quad$ Bottom mounted vertical cylinder $\ldots \ldots \ldots \ldots 2$ 
5.1.1 Solution with three different approaches . . . . . . . . . . . 85

5.1.2 Comparisons considering bichromatic waves . . . . . . . . . . . 93

$5.2 \quad$ Floating cylinder . . . . . . . . . . . . . . . . 99

$5.2 .1 \quad$ Numerical models . . . . . . . . . . . . . . . . . . 101

5.2.2 First-order forces and motions . . . . . . . . . . . . . . . 108

5.2.3 Second-order forces . . . . . . . . . . . . . . . 108

6 ANALYSIS OF THE JPK FLOATING WIND TURBINE . . 115

6.1 Model properties and experimental setup . . . . . . . . 117

6.1.1 Rotor-nacelle-assembly (RNA) . . . . . . . . . . . . . . 119

6.1.2 Mooring system . . . . . . . . . . . . . . . . 119

6.1.3 Environmental conditions . . . . . . . . . . . . . . . 119

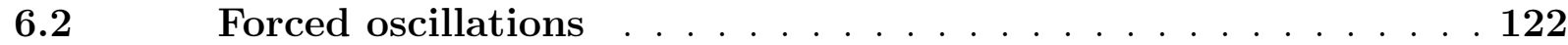

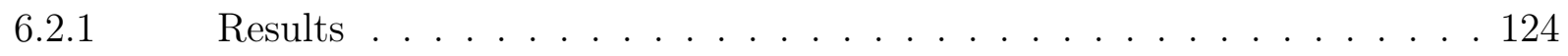

$6.3 \quad$ Numerical models . . . . . . . . . . . . . . . . . . . 128

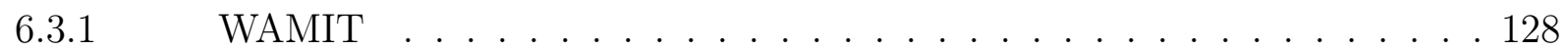

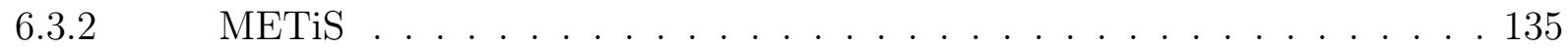

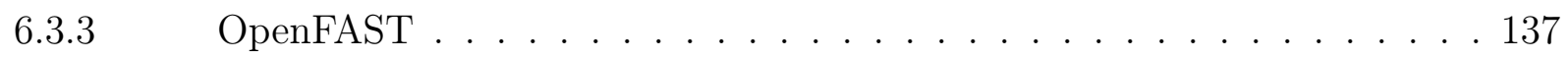

$6.4 \quad$ Decay tests results . . . . . . . . . . . . . . . 139

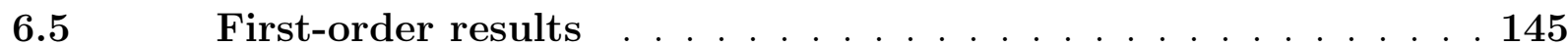

6.5.1 First-order forces due to regular waves . . . . . . . . . . . . . 145

6.5.2 First-order motion RAOs . . . . . . . . . . . . . . . 147

6.6 Second-order surge force and motion under the action of bichromatic waves . . . . . . . . . . . . . . . . . . . . . 150

6.7 Response under the action of irregular waves . . . . . . . . 154

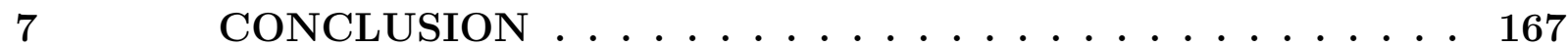

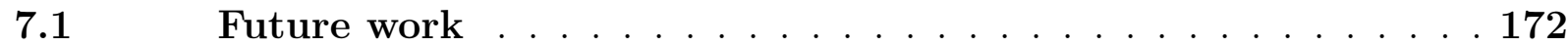

References ................... 175

APPENDIX A-ADDED MASS MATRIX . . . . . . 185

APPENDIX B - ANALYTICAL INTEGRATION OF THE FORCES DUE TO THE LOCAL ACCELERATION .................. 191

APPENDIX C - EXAMPLE OF A METIS INPUT FILE . . 195 
APPENDIX D-SEMI-ANALYTIC SOLUTION FOR THE DIFFERENCE-FREQUENCY SECOND-ORDER WAVE FORCES ON A BOTTOM-MOUNTED VERTICAL CYLINDER . . . . . . . 2 201 APPENDIX E - METHODOLOGY TO POST PROCESS THE FORCED OSCILLATION TESTS . . . . 205 



\section{Introduction}

For centuries, wind power has been harvested for activities such as propelling sailing ships and powering windmills to pump water or grind grains. After the widespread adoption of the coal-fired steam engine in the XVIII century, followed by other fossil fuels, the role played by the wind in human activities was drastically relegated to a low position. However, the increasing awareness of the finiteness of these non-renewable energy sources and of the negative impact they have on the environment has led to a growing interest in renewable energy from many different sources, such as the sun, waves, rain, tides, and wind.

This need for renewable energies, coupled with the high availability of wind and the technical knowledge drawn from other fields, has pushed wind power from a nearly insignificant role in the 1950 s to around $6 \%$ of the global electricity production by the end of 2020 (REN21, 2021). The pace of growth has been fast in the past two decades, especially in the last seven years, in which at least $50 \mathrm{GW}$ of new wind capacity was installed yearly (see Figure 1), in such a way that the worldwide installed capacity of 2020 was more than twice the one from only six years earlier.

Figure 1 - Global cumulative installed wind capacity from 2001 to 2020, including both onshore and offshore capacity.

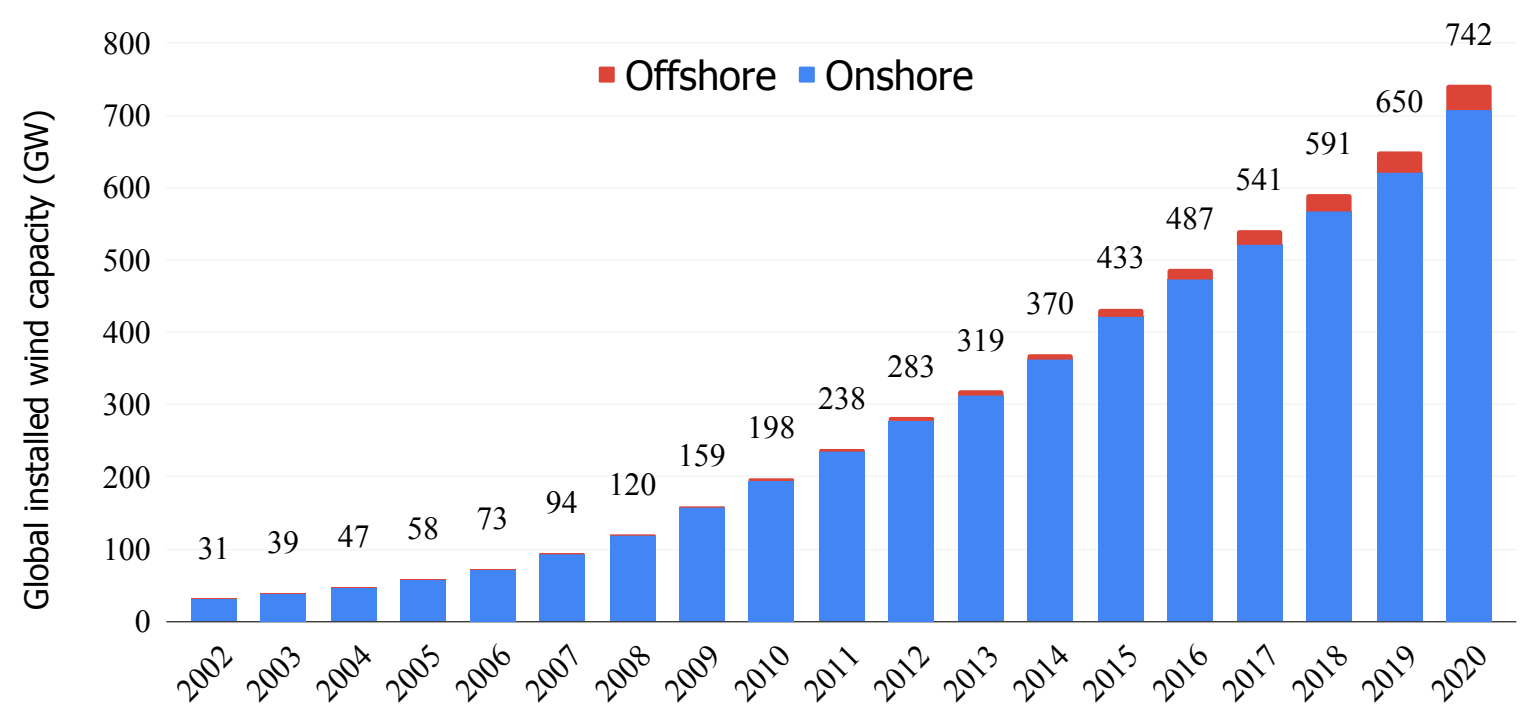

Source: Adapted from GWEC (2021).

Although most of the installed wind capacity is due to onshore wind turbines, the contribution of offshore wind is growing steadily. Around $10 \%$ of the new wind capacity installed in 2019 was offshore $(6.1 \mathrm{GW}$ out of $60.4 \mathrm{GW})$, and by the end of 2020 , almost $5 \%$ 
of the global wind capacity was due to offshore winds (GWEC, 2021). The main advantages of installing wind turbines at sea are the high quality of the wind, which is usually stronger and steadier than onshore, allowing the use of larger and more powerful turbines; the availability of space for the installation of new wind farms; and the reduction of visual and noise pollution. One important disadvantage is the higher cost when compared with onshore wind, mostly due to the foundation of fixed offshore wind turbines (which account for most of the offshore wind capacity), the installation process, and the underwater cables. Nevertheless, as available land is becoming scarcer and wind turbines more powerful, this cost difference is decreasing (GWEC, 2020a). And even though in 2020 the share of new offshore wind capacity was reduced to about $7 \%$ of the total new wind installations, it was a remarkable year because it was the first time that the global capital expenditures committed to offshore wind power exceeded investments in offshore oil and gas (REN21, 2021).

Most of the existing offshore wind turbines are fixed on the sea bottom, a type of solution in which the wind turbine is installed on top of a structure whose foundations are embedded directly into the sea floor. These structures are economically feasible in shallow waters, but costs become prohibitive at waters deeper than around $50 \mathrm{~m}$. Since most of the wind potential is located in deeper water, a solution for harvesting wind energy in these areas is to install the wind turbine on a floating platform, a natural progression that is illustrated in Figure 2. Besides the adequacy for deeper waters, these Floating Offshore Wind Turbines (FOWTs) have the potential to reduce costs by allowing the construction and assembly of the whole system onshore, which can then be towed to the place of installation. This capability is portrayed in Figure 3, which presents pictures of the WindFloat, one of the existing concepts of FOWT, completely assembled in dry dock and then being towed to its final location.

Most of the concepts of floating platforms for FOWTs are directly derived from the oil and gas $(\mathrm{O} \& \mathrm{G})$ industry. They are classified based on how they achieve static stability (BUTTERFIELD et al., 2005), and the most common ones are depicted in Figure 2. The semi-submersible (the fourth from left to right) is stable mainly due to its large water-plane area; the tension leg platform (the next to the right) achieves stability through the tension in the mooring lines, which is due to the structure being lighter than the weight of the displaced water (i.e. excess of buoyancy); while the spar-buoy (last to the right) is heavily ballasted to make its center of mass so close to its bottom that it is below its center of buoyancy. Each concept has its pros and cons, as discussed by James and Ros (2015) and Butterfield et al. (2005).

Several real scale prototypes of FOWTs have been tested. The first one was a 2.3 MW spar called Hywind Demo, installed offshore Karmøy, Norway, in 2009 by Statoil (SKAARE, 2017). In 2011, the first of the aforementioned WindFloat, by Principle 
Figure 2 - Some concepts of offshore wind turbines. The first three on the left are bottomfixed, while the three on the right are the most common floating concepts, namely a semi-submersible, a tension-leg platform (TLP), and a spar.

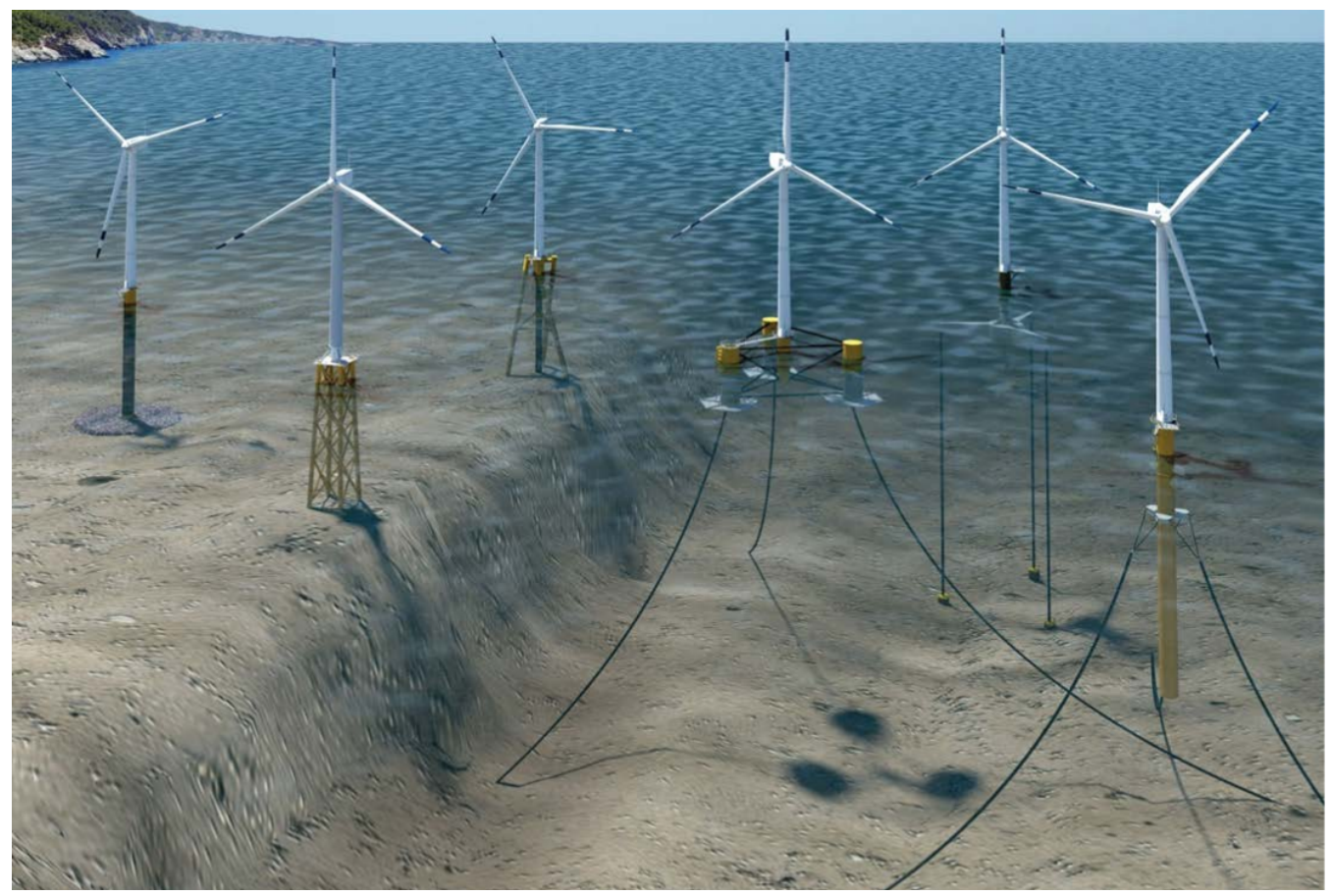

Source: NREL (2015).

Figure 3 - WindFloat in dry dock (left) and being towed to the place of installation (right).
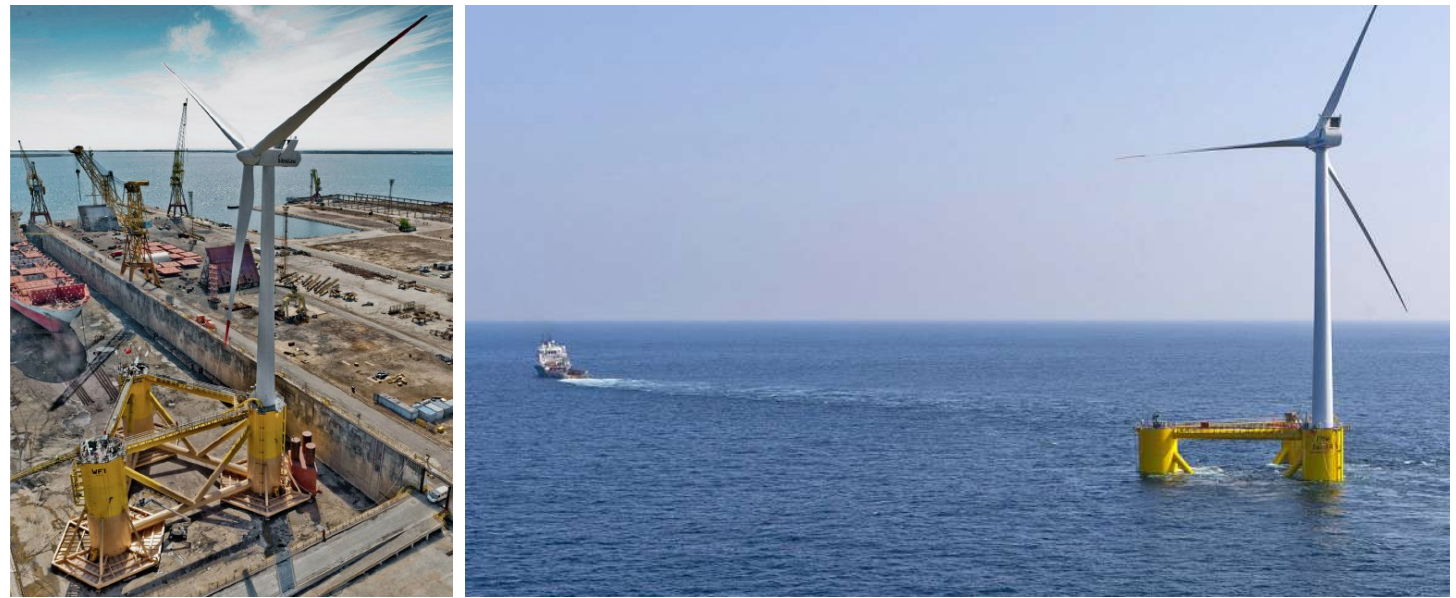

Source: Cermelli and Roddier (2005) and Merrifield (2018).

Power, was inaugurated around $5 \mathrm{~km}$ off the coast of Aguçadoura, Portugal, and was decommissioned in July 2016 (CERMELLI; LEROUX, et al., 2018). After the 2011 Fukushima nuclear disaster, the Japanese government has decided to invest on energy sources other than nuclear, in such a way that three different FOWT concepts and one floating power sub-station have been installed off the coast of Fukushima in an offshore testing site (HITACHI, 2014). These prototypes (and others that were also tested) have withstood harsh environmental conditions, both in terms of waves and wind, proving the 
technical viability of FOWTs.

The current challenge is to reduce costs and prove that floating wind is commercially competitive as a large scale energy source, as demonstrated by the 2030 floating offshore wind forecast given by GWEC (2020b), which ranges from $3 \mathrm{GW}$ to $19 \mathrm{GW}$ depending on how fast the LCOE (levelized cost of energy) can be brought down. An important step was made in October 2017, when the first floating wind farm, Hywind Scotland, was powered up. It was developed by Equinor (formerly Statoil) and is an interesting example to grasp the size of this kind of project. It consists of five $6 \mathrm{MW}$ Hywind floating wind turbines located $25 \mathrm{~km}$ off the coast of Peterhead, Scotland, each with a design draft of $78 \mathrm{~m}$, displacement equal to about $11200 \mathrm{t}$, hub height of $98 \mathrm{~m}$, and rotor diameter of $154 \mathrm{~m}$. The farm covers an area of about $4 \mathrm{~km}^{2}$ with water depths ranging from $95 \mathrm{~m}$ to $120 \mathrm{~m}$. Each turbine is moored using suction anchors and three $2400 \mathrm{~m}$ long mooring lines made of chain. As reported by Equinor, Hywind Scotland achieved a cost reduction of 60 70\% compared with the Hywind Demo project (HARVEY, 2017) (EQUINOR, 2017).

In order to design a cost effective floating wind turbine, a large number of numerical analyses is necessary to assess the performance of different concepts and evaluate which is the best to satisfy the design requirements. These analyses require simulation software to be able to model the coupled dynamics of the whole system, which includes hydrodynamic loads from the waves and sea current, aerodynamic loads from the wind, mooring dynamics, elasticity, and controls of the turbine. For the past few years, a research group at the University of São Paulo has been working on several mathematical models and numerical tools to analyze the different physical aspects pertaining FOWTs, but a single comprehensive software to integrate this independent models has been lacking so far. The starting point of this software is the numerical tool developed in this thesis, called METiS-USP (Morison Equation Time Domain Simulation - University of São Paulo). Though the software needs to include the several aspects required for a comprehensive analysis of floating offshore wind turbines, this thesis is concerned with the hydrodynamics of the floater only, and the development of this in-house code is intended to be continued in the future with the contribution of other members of the research group, who have been studying other topics such as mooring dynamics and elasticity. At the current stage, METiS is capable of evaluating the aerodynamic loads on the rotor with Blade Element Momentum Theory (based on the code developed by Pegoraro (2018)), and the first- and second-order wave loads on the floater using the slender-body approach presented in this thesis.

Due to the high complexity of this kind of tool, a lot of effort has been made to verify and validate these aero-hydro-servo-elastic software. An important initiative are 
the OC3 ${ }^{1}$ (JONKMAN; MUSIAL, 2010), OC4² (ROBERTSON; JONKMAN; VORPAHL, et al., 2014), and OC5 ${ }^{3}$ (ROBERTSON; WENDT, et al., 2017) projects, which have been continuously running since 2005. These projects have engaged a large number of participants from 12 countries and different fields, such as universities, the O\&G and wind industries, research institutes, and certifying agencies, with the aim of comparing the results obtained with diverse simulation software employing different theories and approaches. Although several valuable conclusions have been drawn from the OC projects, some questions regarding the discrepancies among the simulation codes and experimental results are still open. A continuation project, called OC6 ${ }^{4}$, started in 2019 and is planned to last until 2022, demonstrating that there is still work to be done in the development of simulation tools for the design of FOWTs.

One particularly interesting question raised during the OC5 project is about the nonlinear loads due to the waves, as illustrated in Figure 4, which shows the power spectral density of the tower-base shear force for an operational wave excitation (JONSWAP with significant height equal to $7.1 \mathrm{~m}$ and peak period of $12.1 \mathrm{~s}$, without wind nor sea current) calculated by the different participants of the project. Three distinct regions can be distinguished in the graph: the peak around the pitch natural frequency at about $0.03 \mathrm{~Hz}$, the linear wave excitation between $0.07 \mathrm{~Hz}$ and $0.20 \mathrm{~Hz}$, and the region around the tower-bending natural frequency at approximately $0.32 \mathrm{~Hz}$. It is curious that although most of the codes provide fairly good results in the linear wave excitation range, they fail to model the low-frequency response associated with the pitch natural frequency. Since at this frequency there is almost no energy associated with the incoming waves, these loads must come from nonlinear effects, such as the second-order wave forces, thus illustrating their relevance and the difficulty in modeling them.

Among the different methods for the evaluation of wave loads on floating bodies, which are discussed in Chapter 2, the so-called slender-body approach is remarkable for its simplicity. Its application is restricted to hull shapes composed of slender cylinders (i.e. their diameter is small in face of the wave length), a limitation that is satisfied by most of the existing concepts of FOWTs. It is usually implemented using Morison's equation (MORISON et al., 1950), a semi-empirical formulation developed for the determination of wave forces on fixed piles that was later adapted to include the effects of body motions. Due to its original application, Morison's equation neglects some non-linear aspects of the flow that may be important when evaluating the motions of floating structures, given that second-order effects such as mean and slow drift are important. To overcome this limitation, some authors have proposed alternative formulations that include additional

\footnotetext{
${ }^{1}$ Offshore Code Comparison Collaboration

${ }^{2}$ Offshore Code Comparison Collaboration Continuation

${ }^{3}$ Offshore Code Comparison Collaboration, Continued, with Correlation

${ }^{4}$ Offshore Code Comparison Collaboration, Continued, with Correlation and unCertainty
} 
Figure 4 - Power spectral density of the tower-base shear force for an operational wave excitation (JONSWAP with significant height equal to $7.1 \mathrm{~m}$ and peak period of $12.1 \mathrm{~s}$, without wind nor sea current) calculated during the OC5 project by the different participants.

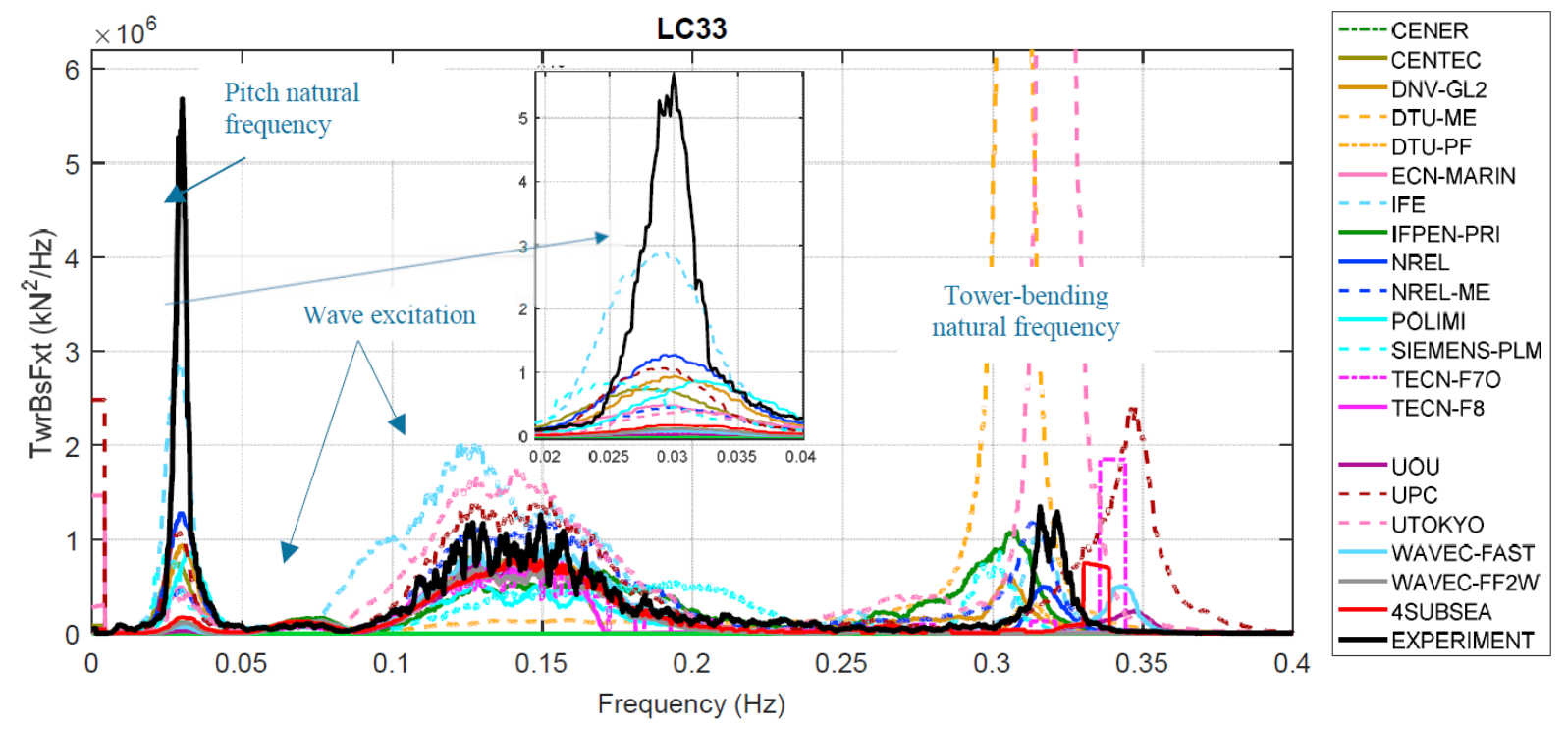

Source: Robertson, Wendt, et al. (2017).

non-linear terms. Based on these works, this thesis proposes a slender-body approach for evaluating the first- and second-order wave forces acting on a floating structure directly in time domain, as opposed to the most common procedure of solving the second-order radiation/diffraction problem in frequency domain and importing the results to timedomain solvers. As a consequence of the slender-body approximation, wave scattering and radiation effects are lost, but it is shown in the text that this is acceptable in several relevant wave conditions.

Though the numerical tool developed in this thesis is focused on FOWTs, it can be used to analyze other offshore structures under the action of waves, provided that they are composed of slender cylinders. For instance, O\&G semi-submersibles may be eligible if the incoming waves are long enough.

\subsection{Objectives}

The main objectives of this thesis are:

i. To understand the physics behind the second-order difference-frequency wave loads acting on a floating structure and model them in time domain using a simple slenderbody approach, evidencing the similarities and differences with respect to diffraction theory. 
The aim is to find an approach that benefits from the geometry of the hull, considered to be composed of slender cylinders, to obtain a simple and fast approach to calculate the second-order difference-frequency wave forces in time domain. This method has the limitation of not being able to model hull shapes that are not exclusively made of slender cylinders, but as this hypothesis can be applied to most of the existing concepts of FOWTs, it is useful after all. In fact, modeling the floater as a slender structure is an approximation that is already employed in the analysis of FOWTs with Morison's equation, and the objective is to extend it to include second-order wave effects using Rainey's formulation (RAINEY, 1989). As shown in the text, the resulting second-order approximation is acceptable roughly for $\lambda / D>10$. When only first-order terms are kept, the formulation is the same as Morison's equation, so the usual threshold of $\lambda / D>5$ is applicable.

ii. To modify the original slender-body approach to make its application to the secondorder hydrodynamic analysis of floating bodies in real seas faster

The slender-body approach independently proposed by Madsen (1986), Rainey (1989), and Manners (1992) evaluates the forces at the instantaneous position of the cylinder, but this is unpractical for numerical simulations with a large number of waves such as the ones required by analyses considering a real sea. Since the position of the body at each time step is unknown at the beginning of the simulation, it would be necessary to directly sum the contribution of each wave component at each time step, which is very computationally expensive. If, however, the forces were evaluated considering the mean body position, it would be possible to use an Inverse Fast Fourier Transform (IFFT) algorithm to compute the necessary time series of wave kinematics much faster. By combining Rainey's equation with Pinkster's formulation (PINKSTER, 1980), the original slender-body approximation can be modified to evaluate the second-order wave loads at the mean body position instead of considering the instantaneous displacements, hence allowing a computationally efficient evaluation of the second-order wave loads in a real sea condition directly in time domain.

iii. To study the suitability of forced oscillation tests to estimate the added mass and drag coefficients, $C_{a}$ and $C_{D}$, which are required by the slender-body approximation. The main challenge when using a slender-body approach, be it via Morison or Rainey's equation, lies in the proper determination of the empirical added mass and drag coefficients, which is an important topic in current research regarding floating wind turbines. These coefficients are dependent on the parameters that characterize the flow, such as the diameter and length of the cylinder, Reynolds number $R e$, Keulegan-Carpenter number $K C$, surface roughness, and proximity to other boundaries. This is a major complication, as each analysis presents different 
combinations of these parameters, hence requiring distinct values of $C_{a}$ and $C_{D}$ that need to be chosen somehow. Due to the resonant nature of the slow-drift motions of floating structures, this choice is of paramount importance, as drag directly impacts the damping levels and, consequently, the amplitude of the slow-drift motion, while the added mass influences the natural periods.

Following the results already published by Carmo, Mello, et al. (2020), Chapter 6 presents the analysis of a FOWT model that was studied during an experimental campaign at the wave basin of the Numerical Offshore Tank of the University of São Paulo (TPN-USP), which included a set of forced oscillation tests of the whole floater to determine the drag and added mass coefficients in different $K C$ numbers and oscillation periods. The results were used as an input to the numerical simulations of the body under the action of waves and wind, and the comparisons between the motions measured in the experiments with the ones calculated numerically are used as a verification of both the slender-body approximation and of the utilization of forced oscillation tests to estimate $C_{a}$ and $C_{D}$. For the latter, however, these should be seen as a preliminary study only, as this is still an ongoing investigation.

iv. To develop a numerical tool for the seakeeping analysis of floating offshore wind turbines that can be useful for the research activities conducted at the University of São Paulo.

This kind of software must include the many different disciplines that take part in the coupled dynamics of a FOWT, and this work is part of a collective effort to build such a tool. The focus here is on the hydrodynamic modeling of the floater, and although other aspects (aerodynamics and mooring) are included in the software, they are done so using either traditional methods or very simplistic approaches, and no contribution is made herein to improve their modeling. These other topics are under study by other members of the research group, and the numerical tool presented here is intended to serve as the basis for the integration of the models that they are working on. The codes developed by Pegoraro (2018), concerning the aerodynamics of wind turbines, and Carmo (2016), which employs Morison's equation to calculate hydrodynamic forces, are taken as an starting point.

\subsection{Text outline}

The text is divided as follows:

- Chapter 2 is dedicated to the literature review, aiming at presenting the works that were important for this thesis as well as an overview about the existing methods for the hydrodynamic analysis of floating wind turbines and offshore structure in general; 
- Chapter 3 presents the derivation of the equations that constitute the slender-body approximation studied in this thesis, with the objective of providing the theoretical background to understand the final expressions of the wave forces acting on the structure and the assumptions that they hold. In short, the floating body is considered to be composed of slender cylinders, in such a way that the second-order wave forces, as given by Pinkster (1980), can be simplified using Rainey's formulation. One important drawback is that wave scattering and radiation effects, as well as end effects due to the extremities of the cylinders, are lost, but it is shown later in the text that this is acceptable in relevant wave conditions;

- Chapter 4 describes the implementation of METiS-USP, the numerical tool that was developed using the method presented in Chapter 3. Besides the hydrodynamic forces, this chapter also presents the inclusion of the aerodynamic loads acting on the rotor using Blade Element Momentum Theory (BEMT) and of the mooring forces with a simple linear stiffness matrix, as well as the numerical scheme used to solve the equations of motion, namely a standard $4^{\text {th }}$ order Runge-Kutta method;

- Chapter 5 compares the results obtained with the slender-body approximation with the ones calculated using diffraction theory and Newman's approximation for the simple case of a single surface piercing cylinder. In the first part of the chapter, the cylinder is considered to be bottom mounted, and the analytical solution given by Kim and Yue (1990) for the low-frequency second-order wave force is used to identify similarities and differences among these different approaches. The same is done in the second half of the chapter, but considering a floating cylinder and the effects that the motions induced by first-order wave forces have on the second-order forces. As this latter case does not have an analytical solution, the radiation/diffraction results are computed with WAMIT ${ }^{\circledR}$ (WAMIT, 2004), a commercial Boundary Element Method (BEM) software for simulating wave interactions with offshore structures in frequency-domain;

- Chapter 6 is about the analysis of JPK, a floating wind turbine that was tested at TPN-USP. Three sets of tests were performed: free decay tests of the moored model; forced oscillations of the hull; and motions under the action of waves (bichromatic, JONSWAP and white-noise) and wind. The model is a semi-submersible with a central column attached to three smaller diameter orbital columns, and it was moored by three catenary lines composed of a bottom segment made of chain and a top one made of polyethylene cable. The same model was then analyzed using METiS, WAMIT and OpenFAST (formerly known as FAST (JONKMAN; BUHL, 2005)), evidencing the capabilities and limitations of each software; 
- The main conclusions are summarized in Chapter 7 along with suggestions for further work. 


\section{Literature Review}

This chapter provides an overview of some works in the field of seakeeping in order to highlight the importance of the low-frequency second-order wave loads and to present the existing methods to evaluate wave forces on floating bodies, specially the so-called slenderbody approximations. Though hydrodynamics is only one of the several disciplines involved in the analysis of floating offshore wind turbines, discussing other topics (aerodynamics, moorings, control, elasticity, etc) is out of the scope of this text, and the interested reader is referred to Jonkman (2007), Manwell, McGowan, and Rogers (2010), Bachynski (2014), Pegoraro (2018) and the references cited by them.

The problem of a floating body under the action of waves is complex and non linear. A first-order approximation is obtained by considering a linearized version of the problem (i.e. the nonlinear boundary conditions on the free surface and on the surface of the body are linearized), in which the loads act at the same frequencies of the incoming waves, $\omega_{j}$, and are linearly proportional to their amplitudes, $A_{j}$. As this approach is capable of solving a large array of relevant seakeeping problems, it was the focus of most of the research on the motions of floating bodies (specially ships) for several decades. As a consequence, methods to estimate first-order wave forces with reasonable accuracy in relevant situations are classical by now, and the ones discussed by Newman (1979) (which includes a very interesting review of the history of seakeeping) are fundamentally the same used nowadays. For instance, the memory function introduced by Cummins (1962) is still applied in most modern time-domain seakeeping software, and the main principles that underline Boundary Element Method (BEM) (discussed ahead) were laid out decades ago (JOHN, 1950; HESS; SMITH, 1967; WEHAUSEN, 1971).

Due to the linearity of the first-order loads, they cover the same range of frequencies of the waves present in the sea, which correspond to periods of a few seconds to about $20 \mathrm{~s}$. To avoid resonant effects, most offshore structures are designed to have resonance periods outside this range (with some notable exceptions, such as wave energy converters). Nevertheless, some response almost always appears at the resonance frequencies, even when practically no wave energy is present at those frequencies, and a mean load is also observed, as first reported by Suyehiro (1924). These phenomena can not be explained by linear theory and, as the motions of the body are well modeled by a linear dynamic system, they can not be due to the equations of motion either. The explanation comes from the next order of approximation of the hydrodynamic problem, which introduces loads acting at the difference, $\omega_{j}-\omega_{l}$, and sum, $\omega_{j}+\omega_{l}$, frequencies. As a consequence, even though these second-order loads are way smaller than the first-order ones, they cover a much wider frequency range that can excite resonances. They are responsible, for instance, for 
the springing of the tendons of tension leg platforms (TLPs) (sum frequencies) and for the slow-drift of moored structures (difference frequencies). Early studies on the second-order loads were connected to the added resistance of ships, which is a problem closely related to the mean drift of a stationary body (see, for instance, Havelock (1942) and Maruo (1957)), but the second-order problem started to draw more attention once the number of structures moored at sea increased. Particularly, the slow-drift motions, which were shown by Remery and Hermans (1972) to be associated with the frequencies of wave groups that are present in irregular waves, became relevant. The reader interested in a review of the first works regarding the mean- and slow-drifts of floating bodies is referred to Pinkster (1980) or Pesce (1984)

\subsection{Main approaches for seakeeping analysis}

There are three main methods for the analysis of wave induced motions: the first is to perform experiments in a wave basin, but as they are usually expensive and time consuming, this solution is not well suited as a recurrent design tool; the second is to solve the problem analytically, an useful approach with the disadvantage of being restricted to a limited number of examples; and the last is to employ numerical methods to solve the equations that describe the flow and the body motions. These methods can be very refined and computationally expensive, such as the Computational Fluid Dynamics (CFD) software that model real fluid flow through the Navier-Stokes equations, or they can use approximations to greatly simplify the problem and obtain faster results. In seakeeping and in many other engineering fields, common practice is to use engineering tools that fit in the latter group, usually validated using analytical results, CFD, and/or experimental tests, and leave more refined analyses to the last stages of design.

This is the case of the numerical tools used to design floating offshore wind turbines, like the ones included in the OC5 project (ROBERTSON; WENDT, et al., 2017). They employ different approximations for each of the topics that concern a FOWT, in such a way that a myriad of combinations is possible. Regarding hydrodynamics modeling, they can be roughly subdivided in three groups based on the assumptions they hold for the calculation of wave loads: the ones that model the floater as a diffracting body; the ones that consider the floater to be composed of slender cylinders; and the ones that combine both approaches.

The theory employed by the first group is based on neglecting viscous effects and rotationality of the flow to evaluate wave loads with potential flow theory. For the problem of an oscillating body, as the ones dealt with in seakeeping, this assumption is true if the ratio of inertial to viscous forces, given by the Reynolds number $\operatorname{Re}=U D / \nu$, is high and if the Keulegan-Carpenter number $K C=\frac{U T}{D}$, related to flow separation, is small 
(FALTINSEN, 1993; SARPKAYA, 2010). This is not always the case, and viscous effects are frequently important, as in the rolling of ships, motions in severe environmental conditions or the slow-drift of moored structures. A simple workaround is to adopt external damping coefficients to partially and heuristically include viscous effects, but since damping is not the only important effect introduced by viscosity, other approaches have been investigated, such as the slender-body approximation discussed later in this chapter.

Although the assumptions required by potential flow theory may seem somewhat strict, it allows the solution of many important problems in hydrodynamics. One of the most valuable ones is the propagation of surface waves, whose solution can be found in Newman (1977) and Dean and Dalrymple (1991). The challenge lies in the nonlinear boundary conditions at the free surface, which is itself unknown, and it has drawn the interest of great minds like Newton, Lagrange, Laplace, Poisson, Cauchy, Green, and Stokes (see Craik (2004) for a fascinating review about the history of water wave theory). For mid and deep water, the solution method relies on a perturbation approach due to Stokes (1847), in which all quantities are decomposed into a power series in the small parameter $\varepsilon=k A$, with $k$ the wave number and $A$ the wave amplitude. The equations are then separated by order, breaking down the original problem into a sequence of linked linear problems, in the sense that the solution of the first-order problem is necessary to solve the second-order problem, which by its turn is used in the solution of the third order problem, and so on. The resulting problems can then be treated by order, allowing the solution of each one separately and the identification of the phenomena related to each of them.

The motion of a floating body under the action of waves can be analyzed using the same technique by including the assumption that body motions are small around their mean position, and a thorough formulation of the problem up to second order may be found in Molin (1979) or Pinkster (1980) (see also Pesce (1984) and Pesce and Aranha (1985) for the rigorous and complete treatment of the problem, up to second-order, for the vertical plane motion case). Despite all the assumed approximations, the problem remains complex when dealing with arbitrary geometries, and this is where the numerical tools that belong to the first group make their contribution. These software usually employ the Boundary Element Method, which is based on a form of Green's theorem that allows the determination of the velocity potential at any point in the fluid from a distribution of singularities over the boundary surfaces, with the intensities of the singularities being determined numerically (FALTINSEN, 1993). This is an important advantage over other methods, as it avoids the discretization of the entire fluid domain. The method traces its origins to Hess and Smith (1967), who originally considered the body to be made of a finite number of plane quadrilateral panels with a continuous distribution of constant intensity sources over each panel. The numerical approach has considerably evolved since them, and it now allows the discretization of the body by plane quadrangular/triangular 
panels, known as low-order panel method, or by the so-called higher order methods, such as using B-spline or NURBS (Non-uniform Rational Basis Spline) (RUGGERI, 2016).

There are several software based on BEM, and many of the most prominent ones, for instance WAMIT ${ }^{\circledR}$ (WAMIT, 2004), AQWA ${ }^{\circledR}$ (ANSYS, 2017), and Hydrostar ${ }^{\circledR}$ (BUREAU VERITAS, 2016), solve the problem in frequency domain, using a Green function that corresponds physically to a pulsating source which automatically satisfies the radiation and boundary conditions on the linear free surface and on the bottom (JOHN, 1950; WEHAUSEN, 1971). Although frequency domain analyses may provide useful information in early stages of design, only time domain analyses are accurate enough to model the coupled nonlinear dynamics of floating wind turbines. Hence, the frequency dependent first- and second- order hydrodynamic coefficients calculated in frequency domain (usually referred to as the solution of the radiation/diffraction problem, illustrated in Figure 5) are imported to a time domain solver, like FAST (JONKMAN; BUHL, 2005) or OrcaFlex (ORCINA, 2021), using Cummins' approach (CUMMINS, 1962; OGILVIE, 1964) for wave radiation effects. An alternative is to use a Boundary Element Method to solve the flow directly in time domain, as the ones described by Korsmeyer, Bingham, and Newman (1999), Watai (2015), and Ruggeri (2016), but they have the disadvantage of being very time consuming, and the author is not aware of any work that employs such methods to the coupled analysis of FOWTs.

Figure 5 - Superposition of the diffraction problem (incoming waves disturbed by the structure, considered to be fixed) with the radiation problem (waves generated by body motions, considering the sea to be perfectly calm).
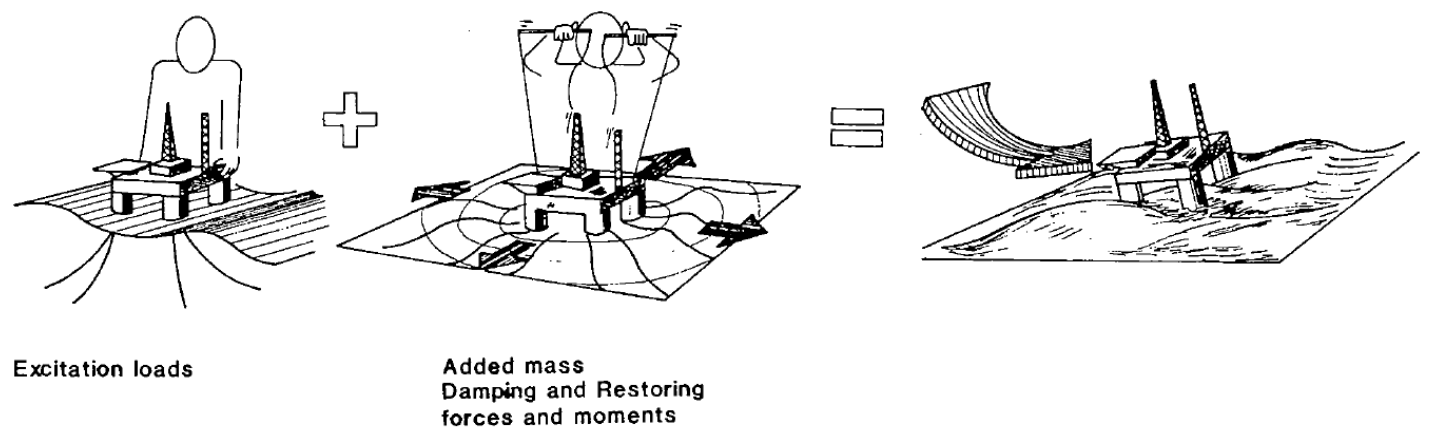

Source: Faltinsen (1993).

The second approach is to consider the body to be composed of slender cylinders under the action of long waves, in the sense that their diameter $D$ is small compared to their draft $d$ and to the incoming wave length $\lambda$. In this condition, it is reasonable to assume that the incoming waves are not disturbed by the structure, so that the flow characteristics (velocity, acceleration, pressure) can be promptly obtained from the undisturbed incoming wave potential. This allows the evaluation of the forces acting on each cylinder by means of specific formulations, with the most common being the one nowadays commonly referred 
to as Morison's equation (MORISON et al., 1950). Morison's equation is a semi-empirical formulation originally developed to calculate the forces exerted by surface waves on piles, but which was later adapted to include the effects of the motions and inclination of the cylinder. It expresses the forces acting on an infinitesimal section of the cylinder as:

$$
\mathrm{d} \mathbf{F}=\rho \frac{\pi D^{2}}{4}\left[\left(1+C_{a}\right) \frac{\partial \mathbf{u}_{P}}{\partial t}-C_{a} \ddot{\mathbf{x}}_{B, P}\right]+\frac{1}{2} \rho D C_{D}\left(\mathbf{u}_{P}-\dot{\mathbf{x}}_{B, P}\right)\left\|\mathbf{u}_{P}-\dot{\mathbf{x}}_{B, P}\right\|
$$

where $\rho$ is the fluid density, $C_{a}$ is the added mass coefficient, $C_{D}$ is the drag coefficient, $\mathbf{u}$ is the flow velocity and $\dot{\mathbf{x}}_{B}$ and $\ddot{\mathbf{x}}_{B}$ are the velocity and acceleration of the body measured at the cylinder section, respectively. The index $P$ indicates the component of the vector that is perpendicular to the cylinder axis. The first and second terms are the inertial hydrodynamic force, proportional to the accelerations, and the third is the drag force, due to viscous effects.

Though Morison's equation looks simple, it hides an intricate question regarding the determination of the added mass and drag coefficients, which need to be chosen somehow. As pointed out by Sarpkaya $(2001,2010)$, since the kinetic velocity of the flow is affected by the flow separation, even the added mass coefficient $C_{a}$ needs to be determined, as it is different from the one calculated considering potential flow. Due to the important role played by cylinders in the offshore industry, there is a large number of experimental works dealing with the case of a cylinder under the action of a sinusoidal flow, such as Keulegan and Carpenter (1958), Sarpkaya (1986), and Venugopal, Varyani, and Barltrop (2006), and it has been found that these coefficients are dependent on the ratio of immersed length to diameter, surface roughness, Keulegan-Carpenter number $K C$, and Reynolds number Re. However, it is not evident how to extend these results to the analysis of a floating structure under the action of a real sea, and how to properly choose $C_{a}$ and $C_{D}$ in this case remains an open question. One possible alternative is to use experimental coefficients obtained in forced oscillation tests in calm waters, and Carmo, Mello, et al. (2020) obtained good results for the slow surge motion of a FOWT under the action of bichromatic waves with coefficients obtained this way. The experimental results analyzed in Chapter 6 are a continuation of that experimental campaign, and as the same approach is followed to obtain the hydrodynamic coefficients of the floater, the utilization of the added mass and drag coefficient from forced oscillations is explained in details in that chapter.

Many of the numerical tools for the analysis of FOWT were developed as an expansion of software that were previously restricted to onshore wind turbines. Probably due to its simplicity and the geometry of offshore wind turbines, Morison's equation was the first approach chosen by most of them to include hydrodynamic loads and expand their capabilities to analyze offshore wind turbines (JONKMAN, 2007). Although being a restrictive approach, since it is not valid for analyzing hull shapes that are not exclusively composed of cylinders, it can be applied to the majority of existing concepts of FOWTs. 
It is worth mentioning that the utilization of this approach is not exclusive to the analysis of FOWTs. For instance, Hooft (1972) deduced an expression for the wave forces on a small body that is very similar to the inertial part of Morison's equation, except for a term that accounts for potential damping that was later neglected for being too small, which was used to analyze the motions of a drilling semi-submersible in waves and validated through comparisons with experimental results. The resulting expression was also compared with the exact analytical results for a bottom mounted cylinder (given by Havelock (1940) for infinite water depth and MacCamy and Fuchs (1954) for finite water depth), leading to an error of less than $4 \%$ when $\lambda / D>5$, which is the usual threshold adopted for the validity of Morison's equation. Another important conclusion was that the influence of free surface effects on the added mass could be neglected.

The third approach, which seems to be the most common, as it is the one followed by most of the participants of the OC5 project (ROBERTSON; WENDT, et al., 2017), consists of a combination of the other two. The hydrodynamic coefficients computed in frequency domain by radiation/diffraction codes are imported to time domain using Cummins' approach, and the quadratic drag term of Morison's equation is added to the other forces. Out of the 21 different software setups studied in the OC5 project, 3 used the first approach, 6 used Morison's equation only, and 12 used a combination of both.

The importance of second-order wave forces in FOWTs has been reported by several authors (COULLING et al., 2013; GUEYDON; DUARTE; JONKMAN, 2014; LOPEZ-PAVON et al., 2015; SIMOS; RUGGERI, et al., 2018), evidencing that they should be taken into account by comprehensive numerical tools for the analysis of floating wind turbines. Just like for oil and gas platforms, one is frequently only interested in the low-frequency part of the second-order wave forces. However, while for O\&G platforms the focus is usually on the low-frequency horizontal motions, with a few exceptions where the vertical degrees of freedom are relevant (for example, the ones discussed by Rezende, Chen, and Ferreira (2007) and Matos, Simos, and Sphaier (2011)), it seems that for FOWTs the vertical motions induced by second-order wave forces are frequently important (e.g. Robertson, Wendt, et al. (2017) and Gueydon, Duarte, and Jonkman (2014)). Besides, while good agreement between experiments and numerical simulations is generally observed for the mean and slow-drift motions of O\&G platforms, important discrepancies have been reported for FOWTs. This is illustrated by the OC5 project (ROBERTSON; WENDT, et al., 2017), during which large differences between numerical and experimental results of the slow motions of a FOWT under the action of waves only have been attributed to the low-frequency second-order loads, and this is currently under investigation in the OC6 project (TOM et al., 2019). Recent studies that have analyzed the same floater with CFD indicate that real flow effects are important not only for the damping of the platform, but also for the low-frequency second-order loads (LI; BACHYNSKI-POLIĆ, 2021a,b,c). 
Most works evaluate the second-order wave loads using radiation/diffraction theory, with time-domain codes either importing the full Quadratic Transfer Functions (QTFs) or using Newman's approximation (NEWMAN, 1974), which consists in evaluating the difference-frequency second-order forces from the mean component, both pre-computed in frequency domain. As the mean wave forces depend only on the solution of the first-order problem, Newman's approximation is extremely useful, but it requires deep water and large natural periods of motion to provide good results. These conditions are usually met by O\&G platforms, but as FOWTs are located in shallower waters with lower natural periods induced by the mooring, Newman's approximation may be unsuitable in many situations (SIMOS; RUGGERI, et al., 2018). Other approximations have been proposed, for example Pinkster's approximation (PINKSTER, 1979), which approximates the secondorder potential by a clever manipulation of the first-order potential, or the white-noise approximation (ARANHA; FERNANDES, 1995; SIMOS; CARMO; CAMARGO, 2018; CARMO; CAMARGO; SIMOS, 2018), which benefits from the narrow bandwidth of the response function of body motions to reduce the required number of frequency pairs of the QTFs.

\subsection{Slender-body approximation for second-order wave loads}

Another line of thought would be to use a slender-body approximation similar to Morison's equation to model the second-order loads, which may be useful in sea conditions where effects due to wave radiation and scattering can be neglected. As the drag term is proportional to the square of the fluid velocity, it is of second-order in wave height, but the inertial term is originally of first-order for being linear with respect to the fluid acceleration. Some works have included additional non-linear effects by considering second-order (or even higher) wave kinematics and wave stretching techniques (ROBERTSON; WENDT, et al., 2017; AGARWAL; MANUEL, 2011), but it has been shown in the past that these are not the only modifications needed to model second-order loads. In fact, in order to solve the forces up to second-order with a slender-body approximation, it would be necessary to replace Morison's inertial term by a more complete expression that is able to describe the potential-flow part of the wave forces up to second-order.

A first improvement follows from Lighthill (1986), who affirms that although in uniform flow the Morison inertia term coincides with potential flow loads, the same is not true for non-uniform flow. In this case, indeed, the "virtual mass force proportional to the horizontal component of the accelerative force exerted on the mass of water displaced by the pile" given by Morison et al. (1950) should include the convective acceleration of the fluid. This is the only change needed for a body that is small (with respect to the non-uniformity of the flow) in all relevant dimensions, for example a small diameter sphere (three dimensional problem) or a small diameter horizontal cylinder parallel to the 
wave crest (two-dimensional problem). Isaacson (1979) extended Morison's equation to include additional nonlinear inertial terms for circular cylindrical piles, but his expression is not correct, as he assumed all body dimensions to be small relative to the length scale, and this is evidenced by a discrepancy with the force derived by Lighthill (1992 apud RAINEY, 1989) ${ }^{1}$ for a fixed circular vertical cylinder, which is correct. Lighthill's result was first generalized to the case of a moving inclined circular cylinder in a nonuniform unsteady potential flow of infinite extent by Madsen (1986), who derived an expression that includes the convective acceleration and two additional terms that are due to the three-dimensionality of the flow. One of them arises from the inclusion of the variation of body velocity along the cylinder axis due to its rotation, while the second one, which was later called axial divergence in other works, comes from including the longitudinal velocity gradient to the water particle acceleration. Manners and Rainey (1992), who analyzed a fixed cylinder in the same flow conditions as Madsen, discuss the axial divergence term in details, demonstrating that its mathematical origin is the three-dimensional feature of a 'zonal harmonic' that produces a convective acceleration. They also present a possible physical interpretation on the grounds that it is related to the rate of extension of the flow in the z-direction, leading to a change in the associated momentum, in such a way that the axial-divergence acceleration acts as if the added mass is changed. The reader is referred to their paper for a more thorough explanation.

Rainey (1989) extended Madsen's result to the case of a cylinder with general cross section using an approach based on energy arguments (Madsen derived his expressions by integrating the pressure on the body surface). Besides the forces along the length of the cylinder, his formulation includes additional point loads at the extremities and at the intersection with the waterline, which are correct when the diameter tends to zero. The rotation term obtained by him is slightly different from the one by Madsen (1986), and in a later work Rainey (1995) asserts that his expression is correct and that there was a factor 2 missing in Madsen's rotation term. This was corroborated by Manners (1992), who obtained the same result as Rainey via pressure integration (thus the same approach followed by Madsen). The resulting expression is deduced in Section 3.3 following the same approach as Manners (1992). This formulation is commonly referred to simply as Rainey's equation (MA; PATEL, 2001; MOLIN; CHEN, 2002; JURADO; BREDMOSE, 2020), unfortunately neglecting the contribution of the other researchers.

Motivated by the fact that the free-surface contribution to the second-order difference-frequency wave force is usually much less important than the contribution from other sources (KIM, 1992), Kim and Chen (1994) employed Rainey's formulation (neglecting the point loads) to develop a slender-body approximation to the computation of second-order difference-frequency wave forces. The incoming flow was described by

\footnotetext{
${ }^{1}$ Lighthill, J. Waves and hydrodynamic loading. BOSS 1979 1st International Conference on the Behaviour of Offshore Structures. London, U.K., 1979.
} 
the analytical difference-frequency second-order incident wave potential, obtained by the solution of the second-order bichromatic wave problem, and they showed that every term in the resulting expression has its counterpart in traditional diffraction theory. The approximation was applied to the analysis of an articulated loading platform, and the resulting pitch moment QTFs were compared with the ones computed with complete second-order diffraction theory, both considering the body fixed. The results agreed very well, except for high frequencies, for which diffraction effects become important. As reported by the authors, their approximation is several orders of magnitude faster than the second-order diffraction method. The effect of body motions is briefly discussed in an appendix of their paper.

A similar approach has been employed by Taylor, Rainey and Dai (1992 apud KIM; CHEN, 1994) $)^{2}$ to the analysis of double frequency wave loads acting on a TLP in regular waves. The results obtained with the slender-body theory agreed with secondorder diffraction theory for survival sea conditions, except, again, for short waves. This disparity was attributed to the free-surface forcing terms present in the second-order sumfrequency diffraction formulation, which in this case are not irrelevant as in the second-order difference-frequency problem. Due to this difference, Kim and Chen (1994) point out that the application of the slender-body approximation to the analysis of sum-frequency wave loads is more restrictive than that for difference-frequency loads.

Other examples of applications of the slender-body approximation are given by Ma and Patel (2001) and Jurado and Bredmose (2020). The former analyzed the second-order forces on an $\mathrm{O} \& \mathrm{G}$ spar platform under the action of regular and bichromatic waves, showing the relevance in some wave conditions of the axial-divergence and rotation terms from the slender-body expressions, while the latter developed an accelerated method to compute second-order inviscid wave loads based on Rainey's equation, but without accounting for the effects of first-order body motions.

Neither of the works cited above used the slender-body approximation to evaluate the response of a floating structure under the action of a real sea directly in time domain, and the author is not aware of any work that has done so. They either neglected body motions (KIM; CHEN, 1994; JURADO; BREDMOSE, 2020) or considered regular/bichromatic waves (TAYLOR; RAINEY; DAI, 1992; MA; PATEL, 2001). This is probably because the formulation proposed by Madsen (1986), Rainey (1989), and Manners (1992) evaluates the forces at the instantaneous position of the cylinder, but this is unpractical for numerical simulations with a large number of waves such as the ones required by analyses considering a real sea. Since the position of the body at each time step is unknown at the beginning of the simulation, it would be necessary to directly sum the contribution of each wave

\footnotetext{
${ }^{2}$ Taylor, R. E.; Rainey, R; Dai, D. Non-linear hydrodynamic analysis of TLP's in extreme waves: Slender body and diffraction theories compared. BOSS 1992 6th International Conference on the Behaviour of Offshore Structures. London, U.K., 1992.
} 
component at each time step, which is very computationally expensive. If, however, the forces were evaluated considering the mean body position, it would be possible to use an Inverse Fast Fourier Transform (IFFT) algorithm to compute the time series of wave kinematics much faster. For this reason, this thesis combines Rainey's equation with the formulation proposed by Pinkster (1980) to modify the original slender-body approximation in order to evaluate the second-order wave loads at the mean body position, hence allowing a computationally efficient evaluation of the second-order wave loads in a real sea condition directly in time domain. 


\section{Slender-body approximation for second- order wave loads}

The numerical tool developed in this thesis employs a slender-body approximation to evaluate the hydrodynamic forces, up to second order, on a floating structure directly in time domain. The advantage is to calculate the hydrodynamic forces concomitantly with the other forces acting on the body, in opposition to the common approach of using hydrodynamic coefficients that need to be pre-computed in frequency domain. Due to the assumptions held by the approximation, this method is well suited to the analysis of floating offshore wind turbines under the action of long waves.

The present chapter is devoted to the derivation of this approximation. It starts with the formulation proposed by Pinkster (1980) for the low-frequency second-order wave forces on floating structures, which are obtained through direct integration of the fluid pressure on the instantaneous wet surface of the body (Section 3.1). This is followed by the description of the incoming wave field in Section 3.2, which is given by the velocity potential of undisturbed waves. As the focus is on the low-frequency second-order loads, it is sufficient to consider the difference-frequency second-order wave potential associated with bichromatic incident waves, as well as the first-order velocity potential of each regular wave component.

The slender-body approximation itself is developed in Section 3.3. The floating body is considered to be composed of an assembly of slender cylinders under the action of long waves, in the sense that their diameter $D$ is small compared to their draft $d$ and to the incoming wave length $\lambda$, a restriction that is satisfied by several of the existing concepts of FOWTs in many relevant sea conditions. The effects of the interaction among the cylinders are disregarded, so that the total hydrodynamic forces acting on the floater are given by the sum of the forces on each cylinder. These hypotheses reduce the problem of studying the flow over a complex geometry to the fundamental one of a cylinder, in such a way that the formulation proposed by Madsen (1986), Rainey (1989), and Manners (1992) for the forces per unit length can be used. As a consequence of the long wave assumption, effects due to wave scattering and radiation are lost in the process, while end effects due to the extremities of the cylinder are discarded by the slenderness approximation. Nevertheless, some of the hydrodynamic loads acting on the extremities of the cylinder are very relevant, specially for the vertical motions of structures with vertical columns, and hence they can not be neglected. This is dealt with in Section 3.3.3 by including the point load at the immersed end proposed by Rainey (1989) and additional axial forces that are similar to the ones usually included when modeling floating structures with Morison's equation (e.g. 
Robertson, Jonkman, Masciola, et al. (2014)).

The slender-body approach, as originally proposed, evaluates the forces at the instantaneous position of the cylinder, which requires fluid kinematics to be computed by directly summing the contribution of each wave component at each time step. This is a computationally expensive operation that makes analyses with a large number of waves, such as the ones considering a real sea, prohibitive. However, by combining Rainey's equation with Pinkster's formulation, the surface integrals presented in Section 3.1 can be simplified into an integration along the length of each member, in such a way that the original slender-body approximation is modified to evaluate the second-order wave loads at the mean body position instead of considering the instantaneous displacements. With this modification, the fluid kinematics that are needed to evaluate the wave loads up to second-order can be computed much faster with an IFFT algorithm, hence allowing the adoption of the slender-body approach even when a large number of wave components is required. This modification is presented in Section 3.3.4.

Section 3.4 discusses viscous effects and their inclusion in the model, done so by adding the quadratic drag term from Morison's equation (MORISON et al., 1950), a semi-empirical superposition of forces that is justified by 70 years of good results obtained with this equation.

Section 3.5 deals with the hydrostatic forces, and it benefits from the simple geometry of a cylinder to calculate the displaced volume and hydrostatic stiffness matrix of the structure without meshing it.

Finally, Section 3.6 presents the final expressions of the hydrodynamic forces, grouping the force components discussed in the previous sections in a more concise way. They are dependent on empirical added mass and drag coefficients, just like Morison's equation, and Section 3.7 discusses the challenge of properly choosing these values for the analysis of floating bodies, an intricate question that remains unsolved despite the many works regarding the issue.

\subsection{Wave forces on floating bodies}

This sections deals with the deduction of the low-frequency second-order wave forces acting on a floating body by direct integration of the pressure on the hull, following the steps presented in Chapter 3 of Pinkster (1980) but with some modifications to the resulting equations in order to solve them with a slender-body approach in time domain.

The theory is developed assuming that the flow is irrotational and the fluid inviscid, homogeneous and incompressible, allowing the fluid velocity $\mathbf{U}$ to be obtained by the 
gradient of a velocity potential $\phi$ :

$$
\mathbf{U}=\nabla \phi(X, Y, Z, t)
$$

where $(X, Y, Z)$ are the coordinates relative to an earth-fixed coordinate system and $t$ is the time. The velocity potential $\phi$ is composed of two parts:

$$
\phi=\varphi+\tilde{\varphi}
$$

with $\varphi$ representing the undisturbed incoming flow and $\tilde{\varphi}$ the perturbation caused by the presence of the body.

Following classical hydrodynamic theory, the equations are derived using perturbation methods. All quantities related to the flow, such as the velocity potential $\phi$, the wave elevation $\eta$, fluid pressure $p$ and body motions $\boldsymbol{\xi}$ can be expanded in a power series with respect to a small parameter $\varepsilon \ll 1$. The fluid pressure, for instance, is written as:

$$
p=p^{(0)}+\varepsilon p^{(1)}+\varepsilon^{2} p^{(2)}+\mathcal{O}\left(\varepsilon^{3}\right)
$$

in which $p^{(0)}$ is the static value, $\varepsilon p^{(1)}$ the first-order perturbation and $\varepsilon^{2} p^{(2)}$ the secondorder one. Thus, first-order quantities are preceded by $\varepsilon$, second-order quantities by $\varepsilon^{2}$, and so on. $\mathcal{O}\left(\varepsilon^{3}\right)$ indicates that higher-order terms are also present in the series expansion, but they will be neglected due to the interest of this work being restricted to second-order effects.

For the problem at hand of a body under the action of waves, the first-order quantities are oscillatory at the frequency of the incoming waves, while second-order quantities contain, in the general case, both low- and high-frequency components. Since the objective is to model the mean and slow-drift forces acting on the structure, the high-frequency components of the second-order solution are of no interest for the present work, and they will be neglected. Hence, the second-order quantities oscillate in a frequency that is lower than the wave frequencies.

Hereafter, the parameter $\varepsilon$ will be omitted in some expressions when only first- or second-order quantities are involved. In these cases, the order of the quantity will still be recognizable by the affix ${ }^{(0)},{ }^{(1)}$ or ${ }^{(2)}$, or by the fact that a component is the product of lower-order quantities. For example, $\left\|\nabla \phi^{(1)}\right\|^{2}$, as the product of two first-order quantities, is recognized as a second-order quantity.

One important remark is that the equations presented hereafter assume that the low-frequency motions induced by the second-order forces are small in relation to the firstorder motions. As pointed out by Pinkster (1980), this is not always true, as low-frequency second-order motions are resonant and, usually, weakly damped, leading to large dynamic magnifications. Nevertheless, if the wave height is assumed to be infinitesimal, i.e. $\varepsilon \rightarrow 0$, the motions induced by the low-frequency second-order forces are guaranteed to be small 
in face of the first-order motions, so this assumption should be kept in mind when studying body motions using second-order wave theory.

\subsubsection{Coordinate systems and body motions}

The following different right-handed coordinate systems are employed, as illustrated in Figure 6:

Figure 6 - Systems of coordinates.

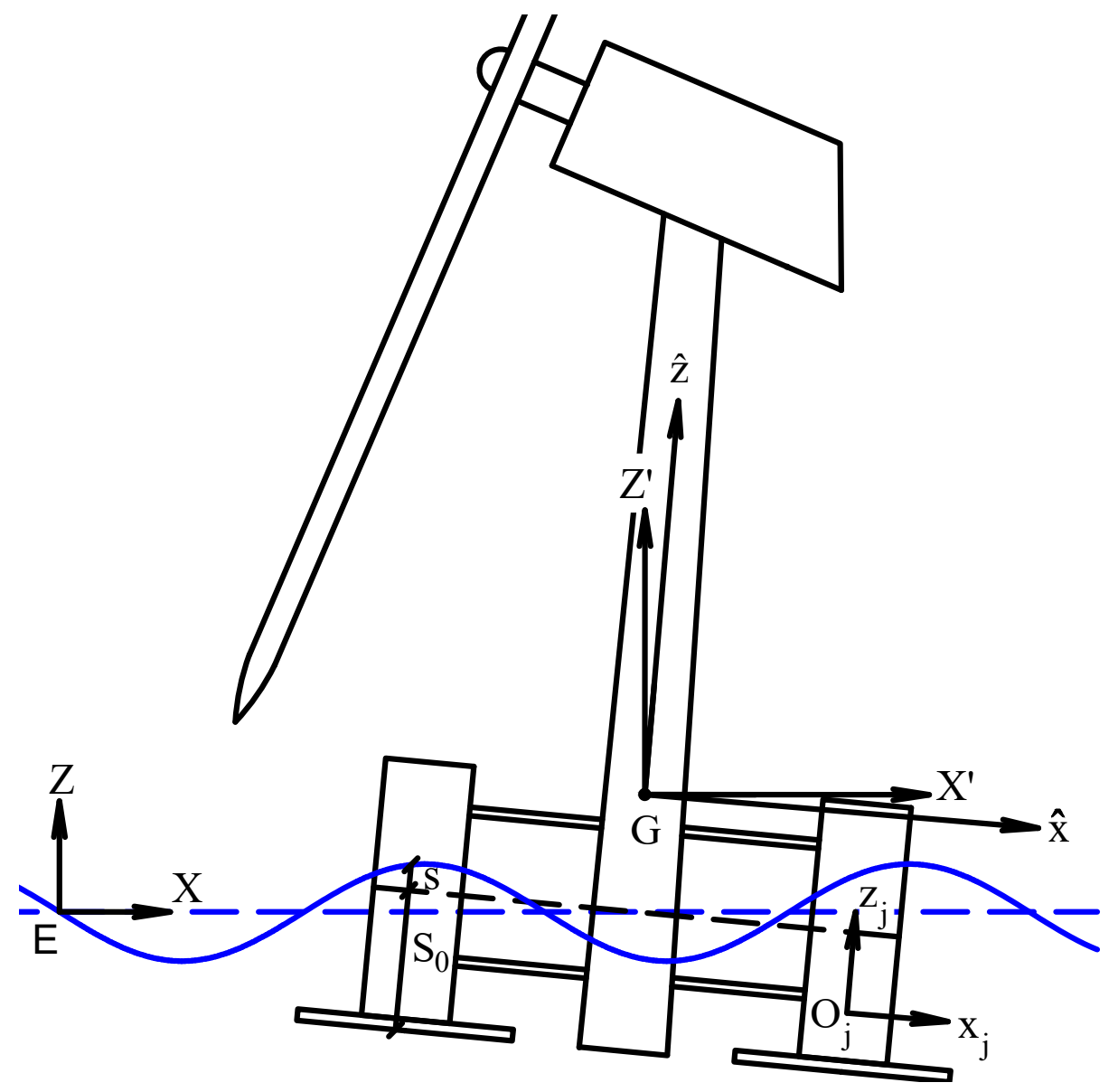

- The earth-fixed coordinate system $E X Y Z$, with the origin $E$ and the axes $X$ and $Y$ lying on the mean free surface. The $Z$ axis is positive upwards. The unit vectors along the axes $(X, Y, Z)$ are denoted $\left\{\mathbf{E}_{1}, \mathbf{E}_{2}, \mathbf{E}_{3}\right\}$;

- The coordinate system $G X^{\prime} Y^{\prime} Z^{\prime}$, with origin in the center of gravity $G$ of the body and axes parallel to those of the $E X Y Z$ system. In other words, it follows the translation of the body, but does not rotate with it;

- The coordinate system $G \hat{x} \hat{y} \hat{z}$ of body-bound axes, with origin in the center of gravity $G$ of the body, $\hat{z}$ axis vertically upwards in the mean position of the vessel and the positive $\hat{x}$ axis in the longitudinal direction. In the mean position, the $\hat{x}, \hat{y}$ and $\hat{z}$ 
axes are parallel to $X, Y$ and $Z$. Basically, it follows the translation and rotation of the body. The unit vectors along the axes $(\hat{x}, \hat{y}, \hat{z})$ are $\left\{\hat{\mathbf{e}}_{1}, \hat{\mathbf{e}}_{2}, \hat{\mathbf{e}}_{3}\right\}$;

- For a body composed of an assembly of cylinders, a coordinate system $O_{j} x_{j} y_{j} z_{j}$ is defined for each cylinder $j$. The axes are oriented by the unit vectors $\mathbf{e}_{3, j}, \mathbf{e}_{2, j}=$ $\mathbf{E}_{3} \wedge \mathbf{e}_{3, j}$ and $\mathbf{e}_{1, j}=\mathbf{e}_{2, j} \wedge \mathbf{e}_{3, j}$, with $\mathbf{e}_{3, j}$ directed along the axis of the cylinder. In these conditions, the inclination of the cylinder with respect to the vertical is given by a rotation around the local $y$ axis, which is quite useful to express some of the equations presented in the following sections. If the cylinder is vertical, this vector basis is flawed, as $\mathbf{E}_{3} \wedge \mathbf{e}_{3, j}$ would be zero, and in this special case the axes are taken as parallel to those of the $E X Y Z$ system. The origin of this coordinate system, $O_{j}$, is taken on different points along the center line of the cylinder as convenient, and this is indicated when $O_{j} x_{j} y_{j} z_{j}$ is used.

The position of a point $P$ on the surface of the hull is described by the vector $\mathbf{r}=(P-G)=r_{1} \hat{\mathbf{e}}_{1}+r_{2} \hat{\mathbf{e}}_{2}+r_{3} \hat{\mathbf{e}}_{3}$, while the orientation of a surface element is given by the outward normal vector $\mathbf{n}=n_{1} \hat{\mathbf{e}}_{1}+n_{2} \hat{\mathbf{e}}_{2}+n_{3} \hat{\mathbf{e}}_{3}$.

If the motions induced by both the first-order and the low-frequency second-order wave forces are small, and neglecting third-order components, the position of the point $P$ is given by:

$$
(P-E)=\mathbf{X}_{P}=\mathbf{X}_{P}^{(0)}+\varepsilon \mathbf{X}_{P}^{(1)}+\varepsilon^{2} \mathbf{X}_{P}^{(2)}
$$

where $\mathbf{X}_{P}^{(0)}$ is the mean position vector, $\mathbf{X}_{P}^{(1)}$ the first-order oscillatory motion and $\mathbf{X}_{P}^{(2)}$ the low-frequency second-order oscillatory motion:

$$
\begin{aligned}
& \mathbf{X}_{P}^{(0)}=\mathbf{X}_{G}^{(0)}+\mathbf{r} \\
& \mathbf{X}_{P}^{(1)}=\mathbf{X}_{G}^{(1)}+\boldsymbol{\alpha}^{(1)} \wedge \mathbf{r} \\
& \mathbf{X}_{P}^{(2)}=\mathbf{X}_{G}^{(2)}+\boldsymbol{\alpha}^{(2)} \wedge \mathbf{r}
\end{aligned}
$$

in which $\mathbf{X}_{G}^{(1)}$ and $\mathbf{X}_{G}^{(2)}$ are the first- and second-order oscillatory motions of the center of gravity, $\boldsymbol{\alpha}^{(1)}$ and $\boldsymbol{\alpha}^{(2)}$ are the first- and second-order oscillatory angular motions of the body, assumed to be small, and $\wedge$ denotes the cross product. For conciseness, the vectors that describe translation and rotation of the body will be aggregated in a single six-component vector $\boldsymbol{\xi}$, which will lighten the notation in some parts of this text:

$$
\begin{aligned}
& \boldsymbol{\xi}_{T}^{(1)}=\xi_{1}^{(1)} \mathbf{E}_{1}+\xi_{2}^{(1)} \mathbf{E}_{2}+\xi_{3}^{(1)} \mathbf{E}_{3}=\mathbf{X}_{G}^{(1)} \\
& \boldsymbol{\xi}_{R}^{(1)}=\xi_{4}^{(1)} \mathbf{E}_{1}+\xi_{5}^{(1)} \mathbf{E}_{2}+\xi_{6}^{(1)} \mathbf{E}_{3}=\boldsymbol{\alpha}^{(1)}
\end{aligned}
$$

with analogous expressions for $\boldsymbol{\xi}^{(2)}$. 
As the outward normal vector $\mathbf{n}$ rotates with the body, it is also an oscillatory quantity with respect to the earth-fixed coordinate system:

$$
\mathbf{n}=\mathbf{n}^{(0)}+\varepsilon \mathbf{n}^{(1)}+\varepsilon^{2} \mathbf{n}^{(2)}
$$

with

$$
\begin{aligned}
& \mathbf{n}^{(1)}=\boldsymbol{\xi}_{R}^{(1)} \wedge \mathbf{n}^{(0)} \\
& \mathbf{n}^{(2)}=\boldsymbol{\xi}_{R}^{(2)} \wedge \mathbf{n}^{(0)}
\end{aligned}
$$

note that $\mathbf{n}^{(0)}$ written in the $E X Y Z$ (or $G X^{\prime} Y^{\prime} Z^{\prime}$ ) coordinate system is simply $\mathbf{n}$ in the $G \hat{x} \hat{y} \hat{z}$ coordinate system, as their axes are parallel at zeroth order. In other words, by denoting $\mathbf{n}^{(0)}=N_{1}^{(0)} \mathbf{E}_{1}+N_{2}^{(0)} \mathbf{E}_{2}+N_{3}^{(0)} \mathbf{E}_{3}$, and reminding that $\mathbf{n}=n_{1} \hat{\mathbf{e}}_{1}+n_{2} \hat{\mathbf{e}}_{2}+n_{3} \hat{\mathbf{e}}_{3}$, it follows that $N_{1}^{(0)}=n_{1}, N_{2}^{(0)}=n_{2}$ and $N_{3}^{(0)}=n_{3}$.

\subsubsection{First- and second-order wave forces}

The hydrodynamic forces and moments acting on the body are obtained through direct integration of the fluid pressure on the instantaneous wet surface of the body (direct pressure integration method):

$$
\begin{aligned}
\mathbf{F} & =-\iint_{S} p \mathbf{n} \mathrm{d} S \\
\mathbf{M}_{G} & =-\iint_{S} p(\mathbf{r} \wedge \mathbf{n}) \mathrm{d} S
\end{aligned}
$$

where the instantaneous wet surface $S$ is composed of the mean wet surface $S_{0}$, which is the surface of the hull below the mean waterline when the structure is at its mean position, and an oscillating part $s=\varepsilon s^{(1)}+\varepsilon^{2} s^{(2)}+\mathcal{O}\left(\varepsilon^{3}\right)$ up to the instantaneous wave profile along the body (see Figure 6). The subscript $G$ in $\mathbf{M}_{G}$ indicates that the moments are calculated with respect to the center of gravity of the body.

The pressure at a point in the fluid domain is given by:

$$
p=p_{\text {atm }}-\rho g Z-\rho \frac{\partial \phi}{\partial t}-\frac{1}{2} \rho\|\nabla \phi\|^{2}+C(t)
$$

with $p_{\text {atm }}$ the atmospheric pressure, $Z$ the vertical position of the point and $C(t)$ a function that depends on time only. Without loss of generality, $p_{\text {atm }}$ and $C(t)$ may be taken as zero (NEWMAN, 1977).

Equations 3.13 and 3.14 require the pressure to be calculated on the surface of the hull, but as those points are part of the body, they undergo first- and second-order 
motions. Applying Bernoulli's equation to the instantaneous position of a point on the surface of the hull yields:

$$
p=-\rho g\left(Z^{(0)}+\varepsilon Z^{(1)}+\varepsilon^{2} Z^{(2)}\right)-\left.\varepsilon \rho \frac{\partial \phi^{(1)}}{\partial t}\right|_{\mathbf{X}(t)}-\left.\varepsilon^{2} \rho \frac{\partial \phi^{(2)}}{\partial t}\right|_{\mathbf{X}(t)}-\varepsilon^{2} \frac{1}{2} \rho\left\|\nabla \phi^{(1)} \mid \mathbf{X}(t)\right\|^{2}
$$

which is not completely of second-order, as the derivatives calculated at the instantaneous position of the point introduce higher-order effects. This can be fixed by applying a Taylor expansion around the mean position of the point:

$$
\begin{aligned}
& \left.\varepsilon \frac{\partial \phi^{(1)}}{\partial t}\right|_{\mathbf{X}(t)}=\left.\varepsilon \frac{\partial \phi^{(1)}}{\partial t}\right|_{\mathbf{X}_{0}}+\left.\varepsilon^{2} \mathbf{X}^{(1)} \cdot \nabla \frac{\partial \phi^{(1)}}{\partial t}\right|_{\mathbf{X}_{0}}+\mathcal{O}\left(\varepsilon^{3}\right) \\
& \left.\varepsilon^{2} \frac{\partial \phi^{(2)}}{\partial t}\right|_{\mathbf{X}(t)}=\left.\varepsilon^{2} \frac{\partial \phi^{(2)}}{\partial t}\right|_{\mathbf{X}_{0}}+\mathcal{O}\left(\varepsilon^{3}\right) \\
& \left.\varepsilon \nabla \phi^{(1)}\right|_{\mathbf{X}(t)}=\left.\varepsilon \nabla \phi^{(1)}\right|_{\mathbf{X}_{0}}+\mathcal{O}\left(\varepsilon^{2}\right)
\end{aligned}
$$

substituting these expressions in Equation 3.16, and retaining only terms up to second order, leads to:

$$
\begin{aligned}
p= & -\rho g\left(Z^{(0)}+\varepsilon Z^{(1)}+\varepsilon^{2} Z^{(2)}\right)-\left.\varepsilon \rho \frac{\partial \phi^{(1)}}{\partial t}\right|_{\mathbf{X}_{0}} \\
& -\left.\varepsilon^{2} \rho \mathbf{X}^{(1)} \cdot \nabla \frac{\partial \phi^{(1)}}{\partial t}\right|_{\mathbf{X}_{0}}-\left.\varepsilon^{2} \rho \frac{\partial \phi^{(2)}}{\partial t}\right|_{\mathbf{X}_{0}}-\varepsilon^{2} \frac{1}{2} \rho\left\|\left.\nabla \phi^{(1)}\right|_{\mathbf{X}_{0}}\right\|^{2}
\end{aligned}
$$

which can be rephrased as

$$
p=p^{(0)}+\varepsilon p^{(1)}+\varepsilon^{2} p^{(2)}
$$

with

$$
\begin{aligned}
& p^{(0)}=-\rho g Z^{(0)} \\
& p^{(1)}=-\rho g Z^{(1)}-\left.\rho \frac{\partial \phi^{(1)}}{\partial t}\right|_{\mathbf{X}_{0}} \\
& p^{(2)}=-\rho g Z^{(2)}-\left.\rho \mathbf{X}^{(1)} \cdot \nabla \frac{\partial \phi^{(1)}}{\partial t}\right|_{\mathbf{X}_{0}}-\left.\rho \frac{\partial \phi^{(2)}}{\partial t}\right|_{\mathbf{X}_{0}}-\frac{1}{2} \rho\left\|\nabla \phi^{(1)} \mid \mathbf{x}_{0}\right\|^{2}
\end{aligned}
$$

Substitution of the pressure $p$ and the normal vector $\mathbf{n}$ in Equation 3.13 leads to:

$$
\begin{aligned}
\mathbf{F}= & -\iint_{S_{0}}\left(p^{(0)}+\varepsilon p^{(1)}+\varepsilon^{2} p^{(2)}\right)\left(\mathbf{n}^{(0)}+\varepsilon \mathbf{n}^{(1)}+\varepsilon^{2} \mathbf{n}^{(2)}\right) \mathrm{d} S \\
& -\iint_{s}\left(p^{(0)}+\varepsilon p^{(1)}+\varepsilon^{2} p^{(2)}\right)\left(\mathbf{n}^{(0)}+\varepsilon \mathbf{n}^{(1)}+\varepsilon^{2} \mathbf{n}^{(2)}\right) \mathrm{d} S
\end{aligned}
$$

resulting in

$$
\mathbf{F}=\mathbf{F}^{(0)}+\varepsilon \mathbf{F}^{(1)}+\varepsilon^{2} \mathbf{F}^{(2)}+\mathcal{O}\left(\varepsilon^{3}\right)
$$


with

$$
\begin{aligned}
\mathbf{F}^{(0)} & =\iint_{S_{0}}-p^{(0)} \mathbf{n}^{(0)} \mathrm{d} S=\iint_{S_{0}} \rho g Z^{(0)} \mathbf{n}^{(0)} \mathrm{d} S=\rho g \mathcal{V}_{0} \mathbf{E}_{3} \\
\mathbf{F}^{(1)} & =\iint_{S_{0}}\left(-p^{(1)} \mathbf{n}^{(0)}-p^{(0)} \mathbf{n}^{(1)}\right) \mathrm{d} S \\
& =\iint_{S_{0}} \rho\left(\frac{\partial \phi^{(1)}}{\partial t}+g Z^{(1)}\right) \mathbf{n}^{(0)} \mathrm{d} S+\boldsymbol{\xi}_{R}^{(1)} \wedge \rho g \mathcal{V}_{0} \mathbf{E}_{3} \\
\mathbf{F}^{(2)} & =\iint_{S_{0}}\left(-p^{(2)} \mathbf{n}^{(0)}-p^{(1)} \mathbf{n}^{(1)}-p^{(0)} \mathbf{n}^{(2)}\right) \mathrm{d} S+\iint_{s^{(1)}}-p^{(1)} \mathbf{n}^{(0)} \mathrm{d} S \\
& =\iint_{S_{0}} \rho\left(\frac{\partial \phi^{(2)}}{\partial t}+\frac{1}{2}\left\|\nabla \phi^{(1)}\right\|^{2}+\mathbf{X}^{(1)} \cdot \nabla \frac{\partial \phi^{(1)}}{\partial t}+g Z^{(2)}\right) \mathbf{n}^{(0)} \mathrm{d} S \\
& +\iint_{S_{0}} \rho\left(\frac{\partial \phi^{(1)}}{\partial t}+g Z^{(1)}\right) \mathbf{n}^{(1)} \mathrm{d} S+\iint_{s^{(1)}} \rho\left(\frac{\partial \phi^{(1)}}{\partial t}+g Z^{(1)}\right) \mathbf{n}^{(0)} \mathrm{d} S+\boldsymbol{\xi}_{R}^{(2)} \wedge \rho g \mathcal{V}_{0} \mathbf{E}_{3}
\end{aligned}
$$

where $\mathcal{V}_{0}$ is the volume of fluid displaced by the body at its mean position. The expressions for the moments are very similar, so they are omitted.

The second-order wave force given by Equation 3.29 leads to the five force components proposed by Pinkster (1980). The procedure is described in his thesis, and results in:

$$
\begin{aligned}
\mathbf{F}^{(2)} & =\iint_{s^{(1)}} \rho\left(\frac{\partial \phi^{(1)}}{\partial t}+g Z^{(1)}\right) \mathbf{n}^{(0)} \mathrm{d} S+\boldsymbol{\xi}_{R}^{(1)} \wedge \mathbf{F}^{(1)} \\
& +\iint_{S_{0}}\left[\frac{1}{2} \rho\left\|\nabla \phi^{(1)}\right\|^{2}+\rho \mathbf{X}^{(1)} \cdot \nabla \frac{\partial \phi^{(1)}}{\partial t}+\rho \frac{\partial \phi^{(2)}}{\partial t}\right] \mathbf{n}^{(0)} \mathrm{d} S
\end{aligned}
$$

The first difference with respect to the expressions presented by Pinkster (1980) is the term $\boldsymbol{\xi}_{R}^{(1)} \wedge \mathbf{F}^{(1)}$ instead of $\boldsymbol{\xi}_{R}^{(1)} \wedge m \ddot{\boldsymbol{\xi}}_{T}^{(1)}$, which is because here other forces may be acting on the body (aerodynamics, mooring, among others), in such a way that the first-order forces can not be replaced by the mass times the first-order acceleration as done by Pinkster. The other difference lies in the term due to the varying wet surface, which in his case is further developed into a line integral at the intersection with the mean waterline, but in here it is better to keep it as shown above for the developments presented in the following sections. The hydrostatic terms are not included in Equation 3.30, except for the integration on $s^{(1)}$, but instead are given separately in Section 3.5 as an hydrostatic stiffness matrix that multiplies the body motions. 
Likewise, the moments are given by:

$$
\begin{aligned}
\mathbf{M}_{G}^{(2)} & =\iint_{s^{(1)}} \rho\left(\frac{\partial \phi^{(1)}}{\partial t}+g Z^{(1)}\right)\left(\mathbf{r} \wedge \mathbf{n}^{(0)}\right) \mathrm{d} S+\boldsymbol{\xi}_{R}^{(1)} \wedge \mathbf{M}_{G}^{(1)} \\
& +\iint_{S_{0}}\left[\frac{1}{2} \rho\left\|\nabla \phi^{(1)}\right\|^{2}+\rho \mathbf{X}^{(1)} \cdot \nabla \frac{\partial \phi^{(1)}}{\partial t}+\rho \frac{\partial \phi^{(2)}}{\partial t}\right]\left(\mathbf{r} \wedge \mathbf{n}^{(0)}\right) \mathrm{d} S
\end{aligned}
$$

These expressions are very general and could be applied to any hull geometry. However, the dynamic pressure remains unknown, as it is still necessary to solve the boundary value problems to obtain the first- and second-order velocity potentials. If the analysis is restricted to bodies composed of slender cylinders and if the interaction among them is neglected, it is possible to solve the boundary value problem using the formulation proposed by Madsen (1986), Rainey (1989), and Manners (1992), in such a way that the surface integrals above are simplified into an integration along the length of each cylinder plus point loads at its extremities, as detailed in the following sections.

\subsection{Description of the incoming wave field}

The incoming flow is due to surface waves freely propagating on the ocean, a classic problem whose solution to second order is well known. Just like in the previous section, the incoming velocity potential $\varphi$ can be expressed as a perturbation series in the small parameter $\varepsilon \ll 1$, which represents the wave steepness, as follows:

$$
\varphi(X, Y, Z, t)=\varepsilon \varphi^{(1)}(X, Y, Z, t)+\varepsilon^{2} \varphi^{(2)}(X, Y, Z, t)+\mathcal{O}\left(\varepsilon^{3}\right)
$$

Since this thesis aims at modeling the second-order difference-frequency wave forces acting on the structure under the action of a real sea, solving the problem up to second-order is enough.

The solution of the first-order problem, known as Airy wave, can be found in Newman (1977), Dean and Dalrymple (1991), or any textbook in marine hydrodynamics. For $N$ waves with complex amplitude $A_{j}$, angular frequency $\omega_{j}$ and propagating with a direction $\beta_{j}$, the first-order wave potential is given by:

$$
\varepsilon \varphi^{(1)}(X, Y, Z, t)=\varepsilon \sum_{j=1}^{N} \varphi_{j}^{(1)}(X, Y, Z, t)=\Re\left\{\sum_{j=1}^{N}-\frac{i g A_{j}}{\omega_{j}} \frac{\cosh \left(k_{j}(Z+h)\right)}{\cosh \left(k_{j} h\right)} e^{i\left(\mathbf{k}_{j} \cdot \mathbf{X}-\omega_{j} t\right)}\right\}
$$

where $\mathbf{X}=X \mathbf{E}_{1}+Y \mathbf{E}_{2}+Z \mathbf{E}_{3}, g$ is the acceleration of gravity, $h$ is the water depth, and $\mathbf{k}_{j}=k_{j}\left(\cos \beta_{j} \mathbf{E}_{1}+\sin \beta_{j} \mathbf{E}_{2}\right)$, with $k_{j}$ the wave number satisfying the dispersion relationship $\omega_{j}^{2}=k_{j} g \tanh \left(k_{j} h\right)$. The mathematical deduction of the expression above 
assumes infinitesimal wave height to deal with the non-linear boundary condition at the free surface, in such a way that Equation 3.33 is valid only for $Z \leq 0$.

While the linearity of the first-order problem allows the solution to be simply the sum of individual regular wave components, this is not the case for the second-order problem, which requires the simultaneous presence of more than one wave component to be taken into account. It is sufficient to consider the combination of bichromatic waves, and the solution is composed of two parts:

$$
\varphi^{(2)}(X, Y, Z, t)=\sum_{j=1}^{N} \sum_{l=1}^{N} \varphi_{j l}^{-}(X, Y, Z, t)+\varphi_{j l}^{+}(X, Y, Z, t)
$$

in which the component $\varphi_{j l}^{-}$is the difference-frequency potential, acting at a frequency $\omega_{j l}^{-}=\omega_{j}-\omega_{l}$, while $\varphi_{j l}^{+}$is the sum-frequency potential, with a frequency $\omega_{j l}^{+}=\omega_{j}+\omega_{l}$. These two components can be solved independently, and the complete solution is presented in Kim and Yue (1990). The well known second-order uniform Stokes wave corresponds to the sum-frequency potential for $j=l$.

As the objective is to model the low-frequency second-order loads, the sum-frequency component is neglected, and only the difference-frequency component $\varphi_{j l}^{-}$is considered. It is given by:

$$
\varphi^{-}=\Re\left\{\sum_{j=1}^{N} \sum_{l=1}^{N} A_{j} A_{l}^{*} \varphi_{j l}^{-} e^{-i\left(\omega_{j}-\omega_{l}\right) t}\right\}
$$

where the asterisk denotes a complex conjugate, $\Re$ the real part and

$$
\begin{gathered}
\varepsilon^{2} \varphi_{j l}^{-}=\frac{1}{2}\left(\gamma_{j l}^{-}+\gamma_{l j}^{-*}\right) \frac{\cosh \left(\left\|\mathbf{k}_{j}-\mathbf{k}_{l}\right\|(z+h)\right)}{\cosh \left(\left\|\mathbf{k}_{j}-\mathbf{k}_{l}\right\| h\right)} e^{i\left(\mathbf{k}_{j}-\mathbf{k}_{l}\right) \cdot \mathbf{x}} \\
\gamma_{j l}^{-}=-\frac{i g}{2 \omega_{j}} \frac{k_{j}^{2}\left[1-\tanh ^{2}\left(k_{j} h\right)\right]-2 k_{j} k_{l}\left[1+\tanh \left(k_{j} h\right) \tanh \left(k_{l} h\right)\right]}{\left(\omega_{j}-\omega_{l}\right)^{2} / g-\left\|\mathbf{k}_{j}-\mathbf{k}_{l}\right\| \tanh \left(\left\|\mathbf{k}_{j}-\mathbf{k}_{l}\right\| h\right)}
\end{gathered}
$$

as for Equation 3.33, Equation 3.35 is valid only for $Z \leq 0$.

One important point is that the expressions above for $\varphi^{(1)}$ and $\varphi^{-}$are written in the global coordinate system, $E X Y Z$, but the results obtained in the following sections require the evaluation of their derivatives with respect to a moving coordinate system such as Oxyz. Any vector q can be converted between these two coordinate systems by the change of basis matrix:

$$
\begin{gathered}
\mathbf{q}=q_{1} \mathbf{e}_{1}+q_{2} \mathbf{e}_{2}+q_{3} \mathbf{e}_{3}=Q_{1} \mathbf{E}_{1}+Q_{2} \mathbf{E}_{2}+Q_{3} \mathbf{E}_{3} \\
{\left[\begin{array}{l}
Q_{1} \\
Q_{2} \\
Q_{3}
\end{array}\right]=\left[\begin{array}{lll}
\mathbf{e}_{1} \cdot \mathbf{E}_{1} & \mathbf{e}_{2} \cdot \mathbf{E}_{1} & \mathbf{e}_{3} \cdot \mathbf{E}_{1} \\
\mathbf{e}_{1} \cdot \mathbf{E}_{2} & \mathbf{e}_{2} \cdot \mathbf{E}_{2} & \mathbf{e}_{3} \cdot \mathbf{E}_{2} \\
\mathbf{e}_{1} \cdot \mathbf{E}_{3} & \mathbf{e}_{2} \cdot \mathbf{E}_{3} & \mathbf{e}_{3} \cdot \mathbf{E}_{3}
\end{array}\right]\left[\begin{array}{l}
q_{1} \\
q_{2} \\
q_{3}
\end{array}\right]}
\end{gathered}
$$


Taking $\mathbf{q}=\mathbf{x}=x \mathbf{e}_{1}+y \mathbf{e}_{2}+z \mathbf{e}_{3}=X \mathbf{E}_{1}+Y \mathbf{E}_{2}+Z \mathbf{E}_{3}$ leads to:

$$
\begin{aligned}
& X=x\left(\mathbf{e}_{1} \cdot \mathbf{E}_{1}\right)+y\left(\mathbf{e}_{2} \cdot \mathbf{E}_{1}\right)+z\left(\mathbf{e}_{3} \cdot \mathbf{E}_{1}\right) \\
& Y=x\left(\mathbf{e}_{1} \cdot \mathbf{E}_{2}\right)+y\left(\mathbf{e}_{2} \cdot \mathbf{E}_{2}\right)+z\left(\mathbf{e}_{3} \cdot \mathbf{E}_{2}\right) \\
& Z=x\left(\mathbf{e}_{1} \cdot \mathbf{E}_{3}\right)+y\left(\mathbf{e}_{2} \cdot \mathbf{E}_{3}\right)+z\left(\mathbf{e}_{3} \cdot \mathbf{E}_{3}\right)
\end{aligned}
$$

So, derivatives with respect to the local coordinate system are equivalent to:

$$
\begin{aligned}
\frac{\partial}{\partial x} & =\frac{\partial}{\partial X} \frac{\partial X}{\partial x}+\frac{\partial}{\partial Y} \frac{\partial Y}{\partial x}+\frac{\partial}{\partial Z} \frac{\partial Z}{\partial x}=\left(\mathbf{e}_{1} \cdot \mathbf{E}_{1}\right) \frac{\partial}{\partial X}+\left(\mathbf{e}_{1} \cdot \mathbf{E}_{2}\right) \frac{\partial}{\partial Y}+\left(\mathbf{e}_{1} \cdot \mathbf{E}_{3}\right) \frac{\partial}{\partial Z} \\
\frac{\partial}{\partial y} & =\frac{\partial}{\partial X} \frac{\partial X}{\partial y}+\frac{\partial}{\partial Y} \frac{\partial Y}{\partial y}+\frac{\partial}{\partial Z} \frac{\partial Z}{\partial y}=\left(\mathbf{e}_{2} \cdot \mathbf{E}_{1}\right) \frac{\partial}{\partial X}+\left(\mathbf{e}_{2} \cdot \mathbf{E}_{2}\right) \frac{\partial}{\partial Y}+\left(\mathbf{e}_{2} \cdot \mathbf{E}_{3}\right) \frac{\partial}{\partial Z} \\
\frac{\partial}{\partial z} & =\frac{\partial}{\partial X} \frac{\partial X}{\partial z}+\frac{\partial}{\partial Y} \frac{\partial Y}{\partial z}+\frac{\partial}{\partial Z} \frac{\partial Z}{\partial z}=\left(\mathbf{e}_{3} \cdot \mathbf{E}_{1}\right) \frac{\partial}{\partial X}+\left(\mathbf{e}_{3} \cdot \mathbf{E}_{2}\right) \frac{\partial}{\partial Y}+\left(\mathbf{e}_{3} \cdot \mathbf{E}_{3}\right) \frac{\partial}{\partial Z}
\end{aligned}
$$

\subsection{Slender-body approximation}

A common practice in offshore engineering is to apply Morison's equation to evaluate wave loads on structures composed of slender cylinders, in the sense that their diameter is small compared to their length and to the length of the incoming waves. By assuming that the hydrodynamic interaction among the cylinders is negligible, it is possible to evaluate the hydrodynamic forces acting on the whole structure by simply summing the forces acting on each cylinder, simplifying the problem significantly.

Morison's equation is composed of two parts: the inertial term, which is proportional to the fluid acceleration, and the quadratic drag term, which is proportional to the square of the fluid velocity. This means that the quadratic drag term is of second-order in wave height, while the inertial term is of first-order (considering the original formulation proposed by Morison et al. (1950), in which only the local acceleration of the flow is taken and a linear incoming wave field is considered). One may be tempted to extend this approach to solve the forces up to second-order by simply considering second-order incoming waves, but this is not strictly correct. As reviewed in Chapter 2, there has been quite a number of works showing that it is necessary to replace the original inertial term from Morison's equation by a more complete expression in order to properly do so. To present this more complete version of Morison's equation, this thesis follows the deduction path described by Manners (1992), who derived the hydrodynamic forces per unit length on a moving circular cylinder in a general potential flow by pressure integration. The procedure is outlined in this section, and the interested reader may find a more detailed description in his paper. The resulting expression for the hydrodynamic forces per unit length are used to simplify 
the surface integrals obtained in Section 3.1 into an integration along the length of each cylinder that is part of the body.

The expression presented by Manners (1992) is identical to the one obtained by Rainey $(1989,1995)$ using an energy conservation approach, except for additional point loads at the immersed end of the cylinder and at the intersection with the free surface, which are not captured by Manners' procedure. The missing force at the immersed end is added to the formulation in Section 3.3.3, along with additional axial forces to partially model the effects of the axial flow, in a similar way to what is usually done when modeling floating structures with Morison's equation (e.g. Robertson, Jonkman, Masciola, et al. (2014)). The point load at the intersection of the cylinder with the free-surface is discarded for being of third-order in wave height for the applications considered in this work.

\subsubsection{Potential flow round a moving cylinder}

The problem at hand is to evaluate the hydrodynamic forces on a cylinder immersed in a general unsteady potential flow. Due to the geometry of the body, it is natural to work with cylindrical coordinates $(r, \theta, z)$, with $x=r \cos \theta, y=r \sin \theta$ and the $(x, y, z)$ axes chosen in such a way that $z$ is parallel to the cylinder axis and $(x, y)=(0,0)$ lies on its center line. Like before, the unit vectors along the axes $(x, y, z)$ are denoted $\left\{\mathbf{e}_{1}, \mathbf{e}_{2}, \mathbf{e}_{3}\right\}$, while the ones along the global fixed axes $(X, Y, Z)$ are $\left\{\mathbf{E}_{1}, \mathbf{E}_{2}, \mathbf{E}_{3}\right\}$. The origin of the local coordinate system, $O$, is taken as the center of the section where the forces will be evaluated, which is an arbitrary point along the center line of the cylinder. The problem is illustrated in Figure 7.

Figure 7 - Segment of length $\mathrm{d} z$ of the cylinder, illustrating the global and local coordinate systems.

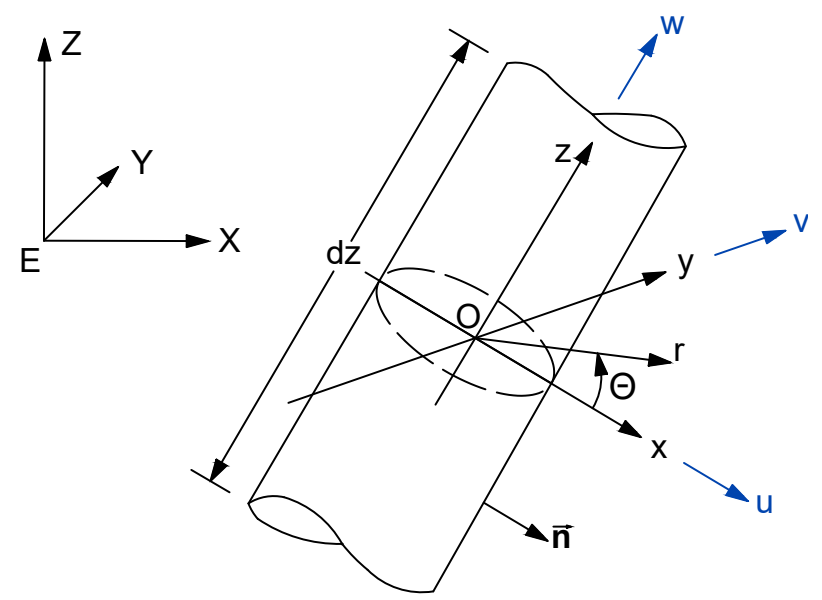

Source: Adapted from Madsen (1986).

Following the same path as Manners (1992) and Manners and Rainey (1992), the velocity potential of the incident flow, $\varphi$, can be expressed as a Taylor series around the 
point $O$ :

$$
\begin{aligned}
\varphi=\varphi_{O} & +\left.\frac{\partial \varphi}{\partial x}\right|_{O} x+\left.\frac{\partial \varphi}{\partial y}\right|_{O} y+\left.\frac{\partial \varphi}{\partial z}\right|_{O} z+\left.\frac{1}{2} \frac{\partial^{2} \varphi}{\partial x^{2}}\right|_{O} x^{2}+\left.\frac{1}{2} \frac{\partial^{2} \varphi}{\partial y^{2}}\right|_{O} y^{2}+\left.\frac{1}{2} \frac{\partial^{2} \varphi}{\partial z^{2}}\right|_{O} z^{2} \\
& +\left.\frac{1}{2} \frac{\partial^{2} \varphi}{\partial x \partial y}\right|_{O} x y+\left.\frac{1}{2} \frac{\partial^{2} \varphi}{\partial x \partial z}\right|_{O} x z+\left.\frac{1}{2} \frac{\partial^{2} \varphi}{\partial y \partial z}\right|_{O} y z
\end{aligned}
$$

where only terms of second order with respect to the cylinder radius were kept (i.e. terms of $\mathcal{O}\left(r^{3}\right)$ were omitted from the expression above). From Laplace's equation, $\partial^{2} \varphi / \partial y^{2}=$ $-\partial^{2} \varphi / \partial x^{2}-\partial^{2} \varphi / \partial z^{2}$. Substituting this relation and using polar coordinates gives:

$$
\begin{aligned}
\varphi=\varphi_{O} & +\left.\frac{\partial \varphi}{\partial z}\right|_{O} z+\left.\frac{1}{4} \frac{\partial^{2} \varphi}{\partial z^{2}}\right|_{O}\left(2 z^{2}-r^{2}\right) \\
& +\left(\left.\frac{\partial \varphi}{\partial x}\right|_{O}+\left.\frac{\partial^{2} \varphi}{\partial x \partial z}\right|_{O} z\right) r \cos \theta+\left(\left.\frac{\partial \varphi}{\partial y}\right|_{O}+\left.\frac{\partial^{2} \varphi}{\partial y \partial z}\right|_{O} z\right) r \sin \theta \\
& +\frac{1}{4}\left(\left.2 \frac{\partial^{2} \varphi}{\partial x^{2}}\right|_{O}+\left.\frac{\partial^{2} \varphi}{\partial z^{2}}\right|_{O}\right) r^{2} \cos 2 \theta+\left.\frac{1}{2} \frac{\partial^{2} \varphi}{\partial x \partial y}\right|_{O} r^{2} \sin 2 \theta
\end{aligned}
$$

The motions of the body are described by the translation velocities of the point $O$, denoted by $\dot{\mathbf{x}}_{B}=\dot{x}_{B} \mathbf{e}_{1}+\dot{y}_{B} \mathbf{e}_{2}+\dot{z}_{B} \mathbf{e}_{3}$, and by the angular velocities $\omega_{x}=-\partial \dot{y}_{B} / \partial z$ and $\omega_{y}=\partial \dot{x}_{B} / \partial z$. As potential flow is assumed, a rotation around the $z$ axis is irrelevant. Hence, the radial velocity of a point on the cylinder surface with coordinates $(r=R, \theta, z)$ is:

$$
\dot{x}_{r}=\left(\dot{x}_{B}+\omega_{y} z\right) \cos \theta+\left(\dot{y}_{B}-\omega_{x} z\right) \sin \theta
$$

The total velocity potential $\phi$ (defined in Equation 3.2) must satisfy the following boundary condition at the surface of the body:

$$
\left.\frac{\partial \phi}{\partial r}\right|_{r=R}=\dot{x}_{r}=\left(\dot{x}_{B}+\omega_{y} z\right) \cos \theta+\left(\dot{y}_{B}-\omega_{x} z\right) \sin \theta
$$

which expresses the impermeability of the body surface. By assuming that the disturbance due to the cylinder, $\tilde{\varphi}$, vanishes with radial distance and that all other boundaries are far enough from the cylinder section that they can be neglected (which is troublesome regarding the free surface, as discussed later), Manners (1992) provides the following solution for the total potential:

$$
\begin{aligned}
\phi=\varphi_{O} & +\left.\frac{\partial \varphi}{\partial z}\right|_{O} z+\left.\frac{1}{4} \frac{\partial^{2} \varphi}{\partial z^{2}}\right|_{O}\left(2 z^{2}-r^{2}+2 R^{2} \ln r\right) \\
+ & {\left[\left.\frac{\partial \varphi}{\partial x}\right|_{O} r+\left.\frac{\partial^{2} \varphi}{\partial x \partial z}\right|_{O} r z+\frac{R^{2}}{r}\left(\left.\frac{\partial \varphi}{\partial x}\right|_{O}-\dot{x}_{B}\right)+z \frac{R^{2}}{r}\left(\left.\frac{\partial^{2} \varphi}{\partial x \partial z}\right|_{O}-\omega_{y}\right)\right] \cos \theta } \\
+ & {\left[\left.\frac{\partial \varphi}{\partial y}\right|_{O} r+\left.\frac{\partial^{2} \varphi}{\partial y \partial z}\right|_{O} r z+\frac{R^{2}}{r}\left(\left.\frac{\partial \varphi}{\partial y}\right|_{O}-\dot{y}_{B}\right)+z \frac{R^{2}}{r}\left(\left.\frac{\partial^{2} \varphi}{\partial y \partial z}\right|_{O}+\omega_{x}\right)\right] \sin \theta }
\end{aligned}
$$




$$
\begin{aligned}
& +\frac{1}{4}\left(\left.2 \frac{\partial^{2} \varphi}{\partial x^{2}}\right|_{O}+\left.\frac{\partial^{2} \varphi}{\partial z^{2}}\right|_{O}\right)\left(r^{2}+\frac{R^{4}}{r^{2}}\right) \cos 2 \theta \\
& +\left.\frac{1}{2} \frac{\partial^{2} \varphi}{\partial x \partial y}\right|_{O}\left(r^{2}+\frac{R^{4}}{r^{2}}\right) \sin 2 \theta
\end{aligned}
$$

which, however, is valid for a coordinate system that is fixed in space, thus it demands a special treatment to be derived with respect to time. The same expression is given by Madsen (1986).

The assumption that the cylinder section is far from any other boundary conditions is good enough for parts of the cylinder that are far from its extremities and from the intersection with the free surface, but it is clearly increasingly wrong as the point $O$ approaches these regions. Rainey $(1989,1995)$ treats this issue rigorously using an energy approach, showing that these end effects generate pressures that are relevant only for a distance of $\mathcal{O}(R)$ around these boundaries, in such a way that they become point loads in the limit $R \rightarrow 0$. Then, if the radius is small in face of the length of the cylinder, it is possible to use the velocity potential $\phi$ given by Equation 3.45 to evaluate the forces along the whole span of the cylinder, while these point loads can be simply added separately.

In this work, only the additional point load at immersed ends of the cylinder is considered (Section 3.3.3), while the point load at the intersection of the cylinder with the free-surface is discarded. The latter originates from the wavy lid assumption that Rainey (1989) introduces to deal with the boundary condition at the free surface. For a vertical cylinder, this load is of third order in wave height, but it is of second order for an oblique surface intersection, and hence should be included in the formulation proposed in this work. However, since most of the surface-piercing elements of FOWTs are vertical or slightly inclined columns, with the exception of structural braces that are usually much more slender than the columns, this load is neglected. This is in line with other works that used Rainey's formulation for the evaluation of low-frequency second-order loads that have neglected the free-surface point load as well (KIM; CHEN, 1994; MA; PATEL, 2001; JURADO; BREDMOSE, 2020). Based on the comments made by Rainey (1995), this load is important, for instance, for problems involving slamming and ringing of offshore structures in steep waves, which are out of the scope of this thesis.

\subsubsection{Forces along the length of the cylinder}

The fluid force acting along the segment of length $\mathrm{d} z$ of the cylinder is given by:

$$
\mathrm{d} \mathbf{F}=\rho \mathrm{d} z \int_{0}^{2 \pi}\left(\frac{\partial \phi}{\partial t}+\frac{1}{2}\|\nabla \phi\|^{2}+g Z\right) \mathbf{n} R \mathrm{~d} \theta
$$

with $\mathbf{n}=\cos \theta \mathbf{e}_{1}+\sin \theta \mathbf{e}_{2}$. For conciseness, the expressions presented in this section will be restricted to the force along the $x$ direction, as the ones for the $y$ direction are very 
similar.

For now, the objective is to obtain the force per unit length acting on the section with center $O, \mathrm{~d} F_{x}$, which, from the expression above, is the sum of the following three integrals:

$$
\begin{aligned}
\mathrm{d} F_{x, \mathrm{i}} & =\mathrm{d} z \int_{0}^{2 \pi} \rho g Z R \cos \theta \mathrm{d} \theta \\
\mathrm{d} F_{x, \mathrm{ii}} & =\mathrm{d} z \int_{0}^{2 \pi} \frac{1}{2} \rho\|\nabla \phi\|^{2} R \cos \theta \mathrm{d} \theta \\
\mathrm{d} F_{x, \mathrm{iii}} & =\mathrm{d} z \int_{0}^{2 \pi} \rho \frac{\partial \phi}{\partial t} R \cos \theta \mathrm{d} \theta
\end{aligned}
$$

The contribution of the hydrostatic pressure field, $\mathrm{d} F_{x, \mathrm{i}}$, is simple to evaluate, yielding:

$$
\mathrm{d} F_{x, \mathrm{i}}=-\rho \pi R^{2} \mathbf{g} \cdot \mathbf{e}_{1} \mathrm{~d} z
$$

with $\mathbf{g}=-g \mathbf{E}_{3}$ the gravitational acceleration.

The part due to the velocity squared requires the following three integrals:

$$
\begin{aligned}
\int_{0}^{2 \pi}\left(\frac{\partial \phi}{\partial r}\right)^{2} \mathrm{~d} \theta & =0 \\
\int_{0}^{2 \pi} \frac{1}{R^{2}}\left(\frac{\partial \phi}{\partial \theta}\right)^{2} \mathrm{~d} \theta & =2 \pi R\left[\left[\left(1+C_{a}\right) u_{O}-C_{a} \dot{x}_{B}\right] \frac{\partial u_{O}}{\partial x}+\left[\left(1+C_{a}\right) v_{O}-C_{a} \dot{y}_{B}\right] \frac{\partial u_{O}}{\partial y}\right. \\
+ & \left.C_{a}\left(u_{O}-\frac{1}{2} \dot{x}_{B}\right) \frac{\partial w_{O}}{\partial z}\right] \\
\int_{0}^{2 \pi}\left(\frac{\partial \phi}{\partial z}\right)^{2} \mathrm{~d} \theta & =2 \pi R\left[w_{O}\left(\left(1+C_{a}\right) \frac{\partial u_{O}}{\partial z}-C_{a} \omega_{y}\right)\right]
\end{aligned}
$$

with $\mathbf{u}_{O}=u_{o} \mathbf{e}_{1}+v_{O} \mathbf{e}_{2}+w_{o} \mathbf{e}_{3}=\left.\nabla \varphi\right|_{o}$ the undisturbed incoming fluid velocity evaluated at the point $O$. The empirical added mass coefficient, $C_{a}$, is introduced multiplying all the terms that originate from the perturbation potential, as usually done with the inertial term of Morison's equation. With the integrals above, the component $\mathrm{d} F_{x, \text { ii }}$ results in:

$$
\begin{aligned}
\mathrm{d} F_{x, \mathrm{ii}}=\rho \pi R^{2}[ & {\left[\left(1+C_{a}\right) u_{O}-C_{a} \dot{x}_{B}\right] \frac{\partial u_{O}}{\partial x}+\left[\left(1+C_{a}\right) v_{O}-C_{a} \dot{y}_{B}\right] \frac{\partial u_{O}}{\partial y} } \\
& \left.+w_{O}\left(\left(1+C_{a}\right) \frac{\partial u_{O}}{\partial z}-C_{a} \omega_{y}\right)+C_{a}\left(u_{O}-\frac{1}{2} \dot{x}_{B}\right) \frac{\partial w_{O}}{\partial z}\right] \mathrm{d} z
\end{aligned}
$$


The component $\mathrm{d} F_{x, \text { iii }}$ can not be so readily evaluated, as the velocity potential $\phi$ given in Equation 3.45 is valid for a space fixed coordinate system. This results in a somewhat lengthy procedure to derive it with respect to time that is not reproduced here, as it does not provide any relevant insight to this text, and the reader is referred to Madsen (1986) and Manners (1992) for a detailed description of the mathematical steps. Fortunately, the final expression is quite concise due to evaluating quantities at the point $O$, for which $z=0$, and to most of the terms being products of trigonometric functions that vanish in the integration from 0 to $2 \pi$ :

$$
\begin{aligned}
\mathrm{d} F_{x, \mathrm{iii}}=\mathrm{d} z \int_{0}^{2 \pi} \frac{\partial \phi}{\partial t} R \cos \theta R \mathrm{~d} \theta=\rho \pi R^{2} & {\left[\left(1+C_{a}\right) \frac{\partial u_{O}}{\partial t}+C_{a}\left(\dot{x}_{B} \frac{\partial u_{O}}{\partial x}+\dot{y}_{B} \frac{\partial u_{O}}{\partial y}-\ddot{x}_{B}\right.\right.} \\
& \left.\left.-\omega_{y}\left(w_{O}-2 \dot{z}_{B}\right)-\frac{1}{2} \dot{x}_{B} \frac{\partial w_{O}}{\partial z}\right)\right] \mathrm{d} z
\end{aligned}
$$

which is the result that would be obtained using Morison's equation plus a rotation and convective-like terms that are second-order and which would be absent if the body was fixed.

The total force per unit length, $\mathrm{d} F_{x}=\mathrm{d} F_{x, \mathrm{i}}+\mathrm{d} F_{x, \text { ii }}+\mathrm{d} F_{x, \text { iii }}$, is then:

$$
\begin{aligned}
\frac{\mathrm{d} F_{x}}{\rho \pi R^{2} \mathrm{~d} z} & =\left(1+C_{a}\right)\left(\frac{\partial u}{\partial t}+u \frac{\partial u}{\partial x}+v \frac{\partial u}{\partial y}+w \frac{\partial u}{\partial z}\right) \\
& -C_{a}\left[\ddot{x}_{B}-\left(u-\dot{x}_{B}\right) \frac{\partial w}{\partial z}+2 \dot{\xi}_{5}\left(w-\dot{z}_{B}\right)\right]-\mathbf{g} \cdot \mathbf{e}_{1}
\end{aligned}
$$

where $\omega_{y}$ was replaced by $\dot{\xi}_{5}$, which is possible because the body is considered rigid, and the index $O$ was omitted to lighten the notation.

Looking at the different terms above, one can readily recognize the inertial part of Morison's equation (the ones with the temporal acceleration, $\partial u / \partial t$, and the body acceleration, $\left.\ddot{x}_{B}\right)$. As could be anticipated, the convective acceleration is present when the slender-body formulation is extended to include higher-order effects, but two additional terms that could not be easily foreseen are also present. The simplest of them is the term $2 \dot{\xi}_{5}\left(w-\dot{z}_{B}\right)$, which is the $\mathrm{x}$-component of the Coriolis acceleration and accounts for the variation of the acceleration along the axis of the cylinder due to its rotation, having fundamentally the same interpretation as the inertial part of Morison's equation. The other one, the so-called axial-divergence term, $\left(u-\dot{x}_{B}\right) \partial w / \partial z$, is not so easily understood, and Manners and Rainey (1992) devote a good deal of their paper to explain its nature and physical interpretation. The authors demonstrate that its mathematical origin is the three-dimensional feature of a 'zonal harmonic' that produces a convective acceleration. They also present a possible physical interpretation on the grounds that $\partial w / \partial z$ represents the rate of extension of the flow in the z-direction, leading to a change in the associated 
momentum, in such a way that the axial-divergence acceleration acts as if the added mass is changed. The reader is referred to their paper for a more thorough explanation and to Kim and Chen (1994) for a brief discussion on a controversy regarding this term.

\subsubsection{Forces at the extremities of the cylinder}

Besides the forces acting along the length of the cylinder, the point loads at an immersed end obtained by Rainey (1989) are added to the formulation:

$$
\begin{aligned}
& F_{\text {end }, x}=C_{a} \rho \pi R^{2}\left(u_{e}-\dot{x}_{e}\right)\left(w_{e}-\dot{z}_{e}\right) \\
& F_{\text {end }, y}=C_{a} \rho \pi R^{2}\left(v_{e}-\dot{y}_{e}\right)\left(w_{e}-\dot{z}_{e}\right) \\
& F_{\text {end }, z}=\left(-\left.\frac{\partial \varphi}{\partial t}\right|_{e}+\rho g Z_{e}\right) \pi R^{2}-\frac{1}{2} C_{a} \rho \pi R^{2}\left[\left(u_{e}-\dot{x}_{e}\right)^{2}+\left(v_{e}-\dot{y}_{e}\right)^{2}\right]
\end{aligned}
$$

where the index $e$ indicates that quantities are evaluated at the extremity of the axis of the cylinder (for a completely submerged cylinder, the forces on both extremities need to be calculated). The forces $F_{\text {end }, x}$ and $F_{\text {end, } y}$ are the same as the one presented by Newman (1977), Section 7.3, for the lateral force on a slender body moving in unbounded still fluid. The force $F_{\text {end, } z}$ is the integration of the fluid pressure over the end face of the cylinder, which for the slender-body limit is simply the pressure evaluated at the center of the circle times its area, and is directed inwards to the body surface. The pressure is taken as the hydrostatic and Froude-Krylov components minus a second-order correction to account for the pressure drop due to the flow velocity (the $(\nabla \phi)^{2}$ term from Bernoulli's equation). In a later work, Rainey (1995) proves that the forces at end loads deduced in Rainey (1989) are actually valid for joints between members as well.

Rainey (1995) treats the end effects as point loads because he assumes that $R \rightarrow 0$, in such a way that the cylinder is reduced to a line with hydrodynamic properties. However, though in some cases the diameter can indeed be considered small compared to the length of the incoming waves, it is usually not sufficiently small in order to completely neglect the added mass associated with its axial motion. For these reasons, the axial force given by Equation 3.59 is modified to include an axial added mass term:

$F_{\text {end }, z}=\left(-\left.\frac{\partial \varphi}{\partial t}\right|_{e}+\rho g Z_{e}\right) \pi R^{2}-\frac{1}{2} C_{a} \rho \pi R^{2}\left[\left(u_{e}-\dot{x}_{e}\right)^{2}+\left(v_{e}-\dot{y}_{e}\right)^{2}\right]+\rho \frac{4 \pi R^{3}}{3} C_{a z}\left(\left.\frac{\mathrm{D} w}{\mathrm{D} t}\right|_{e}-\ddot{z}_{e}\right)$

with $C_{a z}$ the axial added mass coefficient, made dimensionless using the reference volume $4 \pi R^{3} / 3$. This additional term is simply an extrapolation of the approach recommended by Robertson, Jonkman, Masciola, et al. (2014), with the difference that in here the relative acceleration with respect to the body is calculated considering the total water particle acceleration. 
It is important to highlight that the limit $R \rightarrow 0$ assumed by Rainey (1989, 1995) imposes a restriction not only on the ratio between the wave length and cylinder diameter, $\lambda / D$, but also on the ratio between the cylinder length and its diameter, $L / D$. In other words, even if $\lambda / D$ is large enough to neglect wave scattering and radiation, the approximation would perform poorly for a short cylinder (small $L / D$ ), as end effects would not be reasonably modeled by the end loads presented here.

\subsubsection{Modifications to evaluate the forces at the mean body position}

In their formulation, Madsen (1986), Rainey (1989), and Manners (1992) evaluate the forces at the instantaneous position of the cylinder, but, as already mentioned this is unpractical for numerical simulations with a large number of waves such as the ones required by analyses considering a real sea. Alternatively, the expressions for the forces along the length of the cylinder and the point loads at its extremities can be used to simplify the surface integrals presented in Section 3.1, which are evaluated at the mean body position, into integrals along the mean wetted length of the cylinder. As pointed out in that section, this approach requires small body motions, as usually assumed, for example, when using boundary element method to solve the problem.

Following the same reasoning presented in Section 3.1, the first step to do so is to expand the quantities from Equation 3.56 into a power series with respect to $\varepsilon$, i.e. $u=\varepsilon u^{(1)}+\varepsilon^{2} u^{(2)}+\mathcal{O}\left(\varepsilon^{3}\right)$, and again retaining terms only up to second order, leading to the following for the forces along the length of the cylinder:

$$
\begin{aligned}
\frac{\mathrm{d} F_{x}^{(1)}}{\rho \pi R^{2} \mathrm{~d} z} & =\left(1+C_{a}\right) \frac{\partial u^{(1)}}{\partial t}-C_{a} \ddot{x}_{B}^{(1)}-\mathbf{g} \cdot \mathbf{e}_{1}^{(1)} \\
\frac{\mathrm{d} F_{x}^{(2)}}{\rho \pi R^{2} \mathrm{~d} z} & =\left(1+C_{a}\right)\left(\frac{\partial u^{-}}{\partial t}+u^{(1)} \frac{\partial u^{(1)}}{\partial x^{(0)}}+v^{(1)} \frac{\partial u^{(1)}}{\partial x^{(0)}}+w^{(1)} \frac{\partial u^{(1)}}{\partial x^{(0)}}\right) \\
& -C_{a}\left[\ddot{x}_{B}^{(2)}-\left(u^{(1)}-\dot{x}_{B}^{(1)}\right) \frac{\partial w^{(1)}}{\partial z^{(0)}}+2 \dot{\xi}_{5}^{(1)}\left(w^{(1)}-\dot{z}_{B}^{(1)}\right)\right]-\mathbf{g} \cdot \mathbf{e}_{1}^{(2)}
\end{aligned}
$$

in which only the difference-frequency part of the second-order acceleration, $u^{-}$, is considered. As they are evaluated at the mean body position, the forces above are along the mean $x$ axis, whose unit vector is $\mathbf{e}_{1}^{(0)}$.

Integrating $\mathrm{d} F_{x}^{(1)}$ along the mean wetted length of the cylinder, $L_{0}$, yields the following first-order force components:

$$
\begin{aligned}
F_{\phi^{(1)}, x}^{(1)} & =\rho \pi R^{2}\left(1+C_{a}\right) \int_{L_{0}} \frac{\partial u^{(1)}}{\partial t} \mathrm{~d} z \\
F_{\ddot{\mathbf{x}}, x}^{(1)} & =-\rho \pi R^{2} C_{a} \int_{L_{0}} \ddot{x}_{B}^{(1)} \mathrm{d} z
\end{aligned}
$$


where the contribution of the hydrostatic pressure field is not included because it is treated separately in Section 3.5. Likewise, integrating $\mathrm{d} F_{x}^{(2)}$ along $L_{0}$ provides the following second-order forces:

$$
\begin{aligned}
& F_{\phi^{-}, x}^{(2)}=\rho \pi R^{2}\left(1+C_{a}\right) \int_{L_{0}} \frac{\partial u^{-}}{\partial t} \mathrm{~d} z \\
& F_{\mathrm{conv}, x}^{(2)}=\rho \pi R^{2}\left(1+C_{a}\right) \int_{L_{0}}\left(u^{(1)} \frac{\partial u^{(1)}}{\partial x^{(0)}}+v^{(1)} \frac{\partial u^{(1)}}{\partial y^{(0)}}+w^{(1)} \frac{\partial u^{(1)}}{\partial z^{(0)}}\right) \mathrm{d} z \\
& F_{\mathrm{axdv}, x}^{(2)}=\rho \pi R^{2} C_{a} \int_{L_{0}}\left(u^{(1)}-\dot{x}_{B}^{(1)}\right) \frac{\partial w^{(1)}}{\partial z^{(0)}} \mathrm{d} z \\
& F_{\mathrm{rslb}, x}^{(2)}=-\rho \pi R^{2} C_{a} \int_{L_{0}} 2 \dot{\xi}_{5}^{(1)}\left(w^{(1)}-\dot{z}_{B}^{(1)}\right) \mathrm{d} z
\end{aligned}
$$

which correspond to the force components II (pressure drop due to velocity squared) and V (contribution of the second-order potential) from Pinkster (1979). The added mass part of the second-order force is treated separately later.

The same procedure applied to the forces at the extremities of the cylinder yields:

$$
\begin{aligned}
F_{\phi^{(1)}, z}^{(1)} & =-\left.\frac{\partial \varphi^{(1)}}{\partial t}\right|_{e} \pi R^{2}+\left.\rho \frac{4 \pi R^{3}}{3} C_{a z} \frac{\partial w^{(1)}}{\partial t}\right|_{e} \\
F_{\dot{\mathrm{x}}, z}^{(1)} & =-\rho \frac{4 \pi R^{3}}{3} C_{a z} \ddot{z}_{e}^{(1)} \\
F_{\phi^{-}, z}^{(2)} & =-\left.\frac{\partial \varphi^{-}}{\partial t}\right|_{e} \pi R^{2}+\left.\rho \frac{4 \pi R^{3}}{3} C_{a z} \frac{\partial w^{-}}{\partial t}\right|_{e} \\
F_{\mathrm{conv}, z}^{(2)} & =\rho \frac{4 \pi R^{3}}{3} C_{a z}\left(\left.u_{e}^{(1)} \frac{\partial w^{(1)}}{\partial x^{(0)}}\right|_{e}+\left.v_{e}^{(1)} \frac{\partial w^{(1)}}{\partial y^{(0)}}\right|_{e}+\left.w_{e}^{(1)} \frac{\partial w^{(1)}}{\partial z^{(0)}}\right|_{e}\right) \\
F_{\mathrm{end}, z}^{(2)} & =-\frac{1}{2} C_{a} \rho \pi R^{2}\left[\left(u_{e}^{(1)}-\dot{x}_{e}^{(1)}\right)^{2}+\left(v_{e}^{(1)}-\dot{y}_{e}^{(1)}\right)^{2}\right] \\
F_{\mathrm{end}, x}^{(2)} & =C_{a} \rho \pi R^{2}\left(u_{e}^{(1)}-\dot{x}_{e}^{(1)}\right)\left(w_{e}^{(1)}-\dot{z}_{e}^{(1)}\right)
\end{aligned}
$$

As presented in Section 3.1, the effects introduced by body motions are modeled by three force components. The first of them is due to the first-order variation of the normal vector due to the body rotation, which is modeled by rotating the first-order force:

$$
\mathbf{F}_{\mathrm{rotN}}^{(2)}=\boldsymbol{\xi}_{R}^{(1)} \wedge\left(\mathbf{F}_{\phi^{(1)}}^{(1)}+\mathbf{F}_{\mathrm{HS}}^{(1)}\right)
$$

with $\mathbf{F}_{\mathrm{HS}}^{(1)}$ the first-order hydrostatic force (see Section 3.5). This is equivalent to the force component IV from Pinkster (1979), but without replacing the hydrodynamic forces by 
the body mass times its acceleration because other forces may be acting on the body (aerodynamics, mooring, among others).

The second component is caused by the motion of the body in the pressure field, which results in a term containing the product of the first-order motions and the gradient of the first-order pressure (force component III from Pinkster (1979)). For the force along the length of the cylinder, this is equivalent to:

$$
F_{\mathbf{X} \cdot \nabla, x}^{(2)}=\rho \pi R^{2}\left(1+C_{a}\right) \int_{L_{0}} \mathbf{X}^{(1)} \cdot \nabla \frac{\partial u^{(1)}}{\partial t} \mathrm{~d} z
$$

and, for the force acting on the extremities of the cylinder:

$$
\mathbf{F}_{e n d, \mathbf{X} \cdot \nabla, z}^{(2)}=\pi R^{2}\left(-\left.\nabla \frac{\partial \varphi^{(1)}}{\partial t}\right|_{e} \cdot \mathbf{X}_{e}^{(1)}\right)+\rho \frac{4 \pi R^{3}}{3} C_{a z}\left(\left.\nabla \frac{\partial \mathbf{u}^{(1)}}{\partial t}\right|_{e} \cdot \mathbf{X}_{e}^{(1)}\right) \cdot \mathbf{e}_{3}^{(0)}
$$

The last one is due to integrating the first-order forces along the varying wetted length of the cylinder, which requires the evaluation of the incoming wave field above the mean water level. But since the equation that characterizes the first-order incoming flow (Equation 3.33) is deduced assuming infinitesimal wave height, the resulting kinematic field is valid only for $Z \leq 0$. This could be solved by employing a stretching technique, such as Wheeler's stretching (WHEELER, 1970) or other existing method (STANSBERG; GUDMESTAD; HAVER, 2008), that allows the calculation of wave kinematics up to the wave elevation. However, the alternative followed in this work is to perform a Taylor expansion around the mean waterline, $F(Z)=\int_{0}^{Z} f(Z) \mathrm{d} Z=F(0)+f(0) Z+\mathcal{O}\left(Z^{2}\right)$, in a similar way as done by Kim and Chen (1994) and Jurado and Bredmose (2020), yielding:

$$
F_{\eta, x}^{(2)}=\iint_{s^{(1)}} \rho\left(\frac{\partial \phi^{(1)}}{\partial t}+g Z^{(1)}\right) n_{1}^{(0)} \mathrm{d} S=\rho \pi R^{2} \eta_{r}^{(1)}\left[\left.\left(1+C_{a}\right) \frac{\partial u^{(1)}}{\partial t}\right|_{I}-\mathbf{g} \cdot \mathbf{e}_{1}^{(1)}\right]
$$

with $\eta_{r}^{(1)}=\eta_{I}^{(1)}-Z_{I}^{(1)}$ the first-order relative wave elevation evaluated at the intersection of the cylinder axis with the mean waterline, denoted by $I$. This force is traditionally modeled as a waterline integral in diffraction theory and corresponds to component I of Pinkster (1979). It is shown by Rainey (1989) that both approaches provide the same result, and this can be exemplified by a cylinder that is vertical at its mean position, as in this case solving the waterline integral is somewhat simple. Its component along the $x$ direction is:

$$
F_{w l, x}^{(2)}=-\frac{1}{2} \rho g \int_{w l}\left(\eta^{(1)}-Z_{w l}^{(1)}\right)^{2} n_{1}^{(0)} \mathrm{d} l=-\frac{1}{2} \rho g \int_{0}^{2 \pi}\left(\eta^{(1)}-Z_{w l}^{(1)}\right)^{2} R \cos \theta \mathrm{d} \theta
$$

where the first-order elevation is given by $\eta^{(1)}=-\partial \phi^{(1)} / \partial t$ and $Z_{w l}=Z_{I}^{(1)}-\xi_{5}^{(1)} R \cos \theta+$ $\xi_{4}^{(1)} R \sin \theta$. Substitution in the expression above yields:

$$
-\frac{1}{2} \rho g \int_{0}^{2 \pi} \eta^{(1)^{2}} R \cos \theta \mathrm{d} \theta=\left.\rho \pi R^{2}\left(1+C_{a}\right) \eta_{I}^{(1)} \frac{\partial u^{(1)}}{\partial t}\right|_{I}
$$




$$
\begin{aligned}
-\frac{1}{2} \rho g \int_{0}^{2 \pi} Z_{w l}^{(1)^{2}} R \cos \theta \mathrm{d} \theta & =\rho g \pi R^{2} Z_{I}^{(1)} \xi_{5}^{(1)} \\
\rho g \int_{0}^{2 \pi} \eta^{(1)} Z_{w l}^{(1)} R \cos \theta \mathrm{d} \theta & =\rho \pi R^{2} Z_{I}^{(1)}\left[-g \eta_{I}^{(1)} \xi_{5}^{(1)}-\left.(1+C a) \frac{\partial u^{(1)}}{\partial t}\right|_{I} Z_{I}^{(1)}\right] \\
\therefore F_{w l, x}^{(2)} & =\rho \pi R^{2} \eta_{r}^{(1)}\left[\left.\left(1+C_{a}\right) \frac{\partial u^{(1)}}{\partial t}\right|_{I}-g \xi_{5}^{(1)}\right]
\end{aligned}
$$

which is the same as Equation 3.78 for small rotations, as $\mathbf{g} \cdot \mathbf{e}_{1}^{(1)}=-g \mathbf{E}_{3} \cdot \mathbf{e}_{1}^{(1)} \approx g \xi_{5}^{(1)}$.

One important absence in the second-order forces above is the contribution of the body acceleration, $\mathbf{F}_{\ddot{\mathbf{x}}}^{(2)}$, and the reason for this is that a different approach is followed for this force component. The acceleration of a point on the body is related to the rigid body motions of the structure by:

$$
\ddot{\mathbf{x}}_{B}=\ddot{\boldsymbol{\xi}}_{T}+\ddot{\boldsymbol{\xi}}_{R} \wedge \mathbf{r}+\dot{\boldsymbol{\xi}}_{R} \wedge\left(\dot{\boldsymbol{\xi}}_{R} \wedge \mathbf{r}\right)
$$

where the first two terms are linearly proportional to the accelerations of the body, $\ddot{\mathbf{x}}_{l}=$ $\ddot{\boldsymbol{\xi}}_{T}+\ddot{\boldsymbol{\xi}}_{R} \wedge \mathbf{r}$, and the last term is the centripetal acceleration of the point, $\ddot{\mathbf{x}}_{\text {ctp }}=\dot{\boldsymbol{\xi}}_{R} \wedge\left(\dot{\boldsymbol{\xi}}_{R} \wedge \mathbf{r}\right)$. As described in Appendix A, the component $\ddot{\mathbf{x}}_{l}$ yields a force and a moment that can be written as the product of an added mass matrix and the body acceleration vector. In other words, the first-order load due to the body acceleration, given by the sum of Equations 3.64 and 3.70 , can be stated as:

$$
\mathcal{F}_{\ddot{\mathbf{x}}}^{(1)}=-\mathbb{A} \ddot{\boldsymbol{\xi}}^{(1)}
$$

with $\mathbb{A}=\mathbb{A}^{(0)}$ the added mass matrix of the body calculated with the expressions of Appendix A considering the mean body position and $\mathcal{F}_{\ddot{\mathbf{x}}}^{(1)}=\left[\mathbf{F}_{\ddot{\mathbf{x}}}^{(1)} ; \mathbf{M}_{G, \ddot{\mathrm{x}}}^{(1)}\right]$ a six-component vector that groups the forces and moments with respect to the center of gravity. The equivalent second-order load is given by:

$$
\mathcal{F}_{\ddot{\mathbf{x}}}^{(2)}=-\left(\mathbb{A}_{T}^{(1)}-\mathbb{A}\right) \ddot{\boldsymbol{\xi}}^{(1)}-\mathbb{A}^{(2)}+\mathcal{F}_{c t p}^{(2)}
$$

with $A_{T}^{(1)}$ the time varying added mass matrix, also calculated with the expressions of Appendix A but considering the instantaneous first-order position of the body instead of its mean position. The first term takes into account the first-order variable wetted length and the first-order variation of the normal vector simply by taking the difference between $A_{T}^{(1)}$, which can be easily evaluated at each time step, and $\mathbb{A}$. The second term is equivalent to Equation 3.85, but considering the second-order motion of the body, while the last one is the force due to the centripetal acceleration. This approach is followed because the evaluation of the forces due to the body acceleration does not require fluid kinematics, hence the added mass matrix can be evaluated at the instantaneous first-order 
body position (the term $\mathbb{A}_{T}^{(1)}$ ) without significant computational cost. Thus, the procedure introduced above to evaluate the forces at the mean body position is not necessary for this force component.

The loads due to the centripetal acceleration, $\mathcal{F}_{c t p}^{(2)}$, depend on the velocity of the body and are as follows along the length of the cylinder

$$
\mathcal{F}_{c t p, x}^{(2)}=-\int_{L_{0}} \rho \pi R^{2} C_{a}\left(\ddot{\mathbf{x}}_{\mathrm{ctp}}^{(1)} \cdot \mathbf{e}_{1}^{(0)}\right) \mathbf{e}_{1}^{(0)} \mathrm{d} z
$$

and as follows for the contribution at the cylinder extremities:

$$
\mathcal{F}_{c t p, z}^{(2)}=-\rho \frac{4 \pi R^{3}}{3}\left[C_{a z, b} \ddot{\mathbf{x}}_{\mathrm{ctp}, e}^{(1)} \cdot \mathbf{e}_{3}^{(0)}\right] \mathbf{e}_{3}^{(0)}
$$

These expressions for the forces due to the acceleration of the body are particularly useful because they make it easy to isolate the terms containing $\ddot{\xi}$ from the others, allowing the equations of motion to be written as $\dot{\mathbf{q}}=\mathbf{f}(t, \mathbf{q})$ and numerically integrated directly, as detailed in Chapter 4. More specifically, the part of $\mathcal{F}_{\ddot{\mathbf{x}}}^{(1)}$ and $\mathcal{F}_{\ddot{\mathbf{x}}}^{(2)}$ given by $\ddot{A}^{(1)}$ and $\mathbb{A} \ddot{\boldsymbol{\xi}}^{(2)}$ are grouped with the inertia of the body in the left side of the equations of motion, while the other components are kept as forcing terms on the right side.

To ease the notation later in this text, the part of $\mathcal{F}_{\ddot{\mathbf{x}}}^{(2)}$ that is due to the first-order variation of the added mass matrix is denoted by:

$$
\mathcal{F}_{A_{T}^{(1)}}^{(2)}=-\left(\mathbb{A}_{T}^{(1)}-\mathbb{A}\right) \ddot{\boldsymbol{\xi}}^{(1)}
$$

The expressions for the moments corresponding to the force components above can be obtained simply by including the cross product between the force per unit length and the vector $\mathbf{r}=(P-G)$, where $P$ is the integration point.

\subsection{Viscous effects}

The hydrodynamic forces presented in the previous sections were obtained considering potential flow ${ }^{1}$, and, consequently, they result in zero net force (though not necessarily zero net moment) if the flow is permanent and uniform, a clear contradiction with experimental evidence that is classically known as d'Alembert's paradox. This is actually no paradox at all, but simply a consequence of neglecting fluid viscosity and the associated flow separation. Following the model proposed by Morison et al. (1950), a simple workaround is to add a quadratic drag force to the forces obtained in the previous sections. It consists

\footnotetext{
${ }^{1}$ Strictly true if the added mass coefficient was taken as $C_{a}=1$, but slender-body approaches such as Morison's equation and Rainey's formulation are frequently applied with $C_{a}$ obtained in conditions where viscous effects are relevant, thus incorporating indirectly these effects in the evaluation of the inertial forces.
} 
of a force per unit length along the cylinder, which acts perpendicularly to its axis, plus an axial drag force concentrated at each extremity:

$$
\begin{aligned}
& \mathbf{F}_{\mathrm{drag}, P}=\int_{L_{0}} \frac{1}{2} \rho D C_{D}|| \mathbf{u}_{P}^{(1)}-\dot{\mathbf{x}}_{P} \|\left(\mathbf{u}_{P}^{(1)}-\dot{\mathbf{x}}_{P}\right) \mathrm{d} z \\
& \mathbf{F}_{\mathrm{drag}, z}=\frac{1}{2} \rho \pi R^{2} C_{D z}\left|w_{e}^{(1)}-\dot{z}_{B}\right|\left(w_{e}^{(1)}-\dot{z}_{B}\right) \mathbf{e}_{3}^{(0)}
\end{aligned}
$$

with $\mathbf{u}_{P}^{(1)}=u^{(1)} \mathbf{e}_{1}^{(0)}+v^{(1)} \mathbf{e}_{2}^{(0)}, \dot{\mathbf{x}}_{P}=\dot{x}_{B} \mathbf{e}_{1}^{(0)}+\dot{y}_{B} \mathbf{e}_{2}^{(0)}, w_{e}^{(1)}$ the axial fluid velocity calculated at the corresponding end point, $C_{D}$ the empirical drag coefficient associated with the flow perpendicular to the cylinder and $C_{D z}$ the axial drag coefficient of the extremity of the cylinder. Note that the total velocity of the body is considered in order to damp the motions induced by both the first- and second-order forces, and though this approach leads to the inclusion of higher-order terms, it can be seen as an extension to the current practice of using the quadratic drag term in first-order time domain simulations. As the drag force is a higher-order quantity, the expressions above are evaluated considering the mean body position.

\subsection{Hydrostatic forces}

The forces originating from the hydrostatic pressure field, which is the one exhibited by the fluid when it is at rest, are responsible for the equilibrium and stability of a floating body, constituting one of the oldest and most fundamental disciplines of naval architecture. In principle, they are quite simple: the hydrostatic force acting on a body, be it floating or submerged, is the force that was acting at the volume of fluid that had to be displaced to make room for the body. Hence, its magnitude is equal to the weight of displaced fluid; it is directed upwards; and its center of application is equal to the center of mass of the displaced fluid. This fundamental result of fluid mechanics is known as Archimedes' principle.

The same conclusion is obtained by developing the hydrostatic terms in Equations 3.27, 3.28 and 3.29. The procedure is detailed in Newman (1977), Lee (1995) and in numerous textbooks on marine hydrodynamics. The resulting zeroth order contribution is:

$$
\begin{aligned}
\mathbf{F}_{H S}^{(0)} & =\rho g \mathcal{V}_{0} \mathbf{E}_{3} \\
\mathbf{M}_{H S}^{(0)} & =\rho g \mathcal{V}_{0}\left(\hat{y}_{b} \mathbf{E}_{1}-\hat{x}_{b} \mathbf{E}_{2}\right)
\end{aligned}
$$

where $\mathcal{V}_{0}$ is the volume of fluid displaced by the body at its mean position and $\hat{x}_{b}$ and $\hat{y}_{b}$ are the coordinates of the center of buoyancy in the $G \hat{x} \hat{y} \hat{z}$ coordinate system. If the body is at equilibrium, the center of buoyancy is vertically aligned with the center of gravity, hence $\hat{x}_{b}$ and $\hat{y}_{b}$ are zero in that case (it is worth reminding that the origin of the local coordinate system is at the center of gravity). 
Assuming that body motions are small (as already done in Section 3.1), the firstorder hydrostatic forces and motions are equal to a symmetric stiffness matrix that multiplies the first order motions, $\mathcal{F}_{H S}^{(1)}=-\mathbb{K}_{H S} \boldsymbol{\xi}^{(1)}$, given by:

$$
\mathbb{K}_{H S}=\rho g\left[\begin{array}{cccccc}
0 & 0 & 0 & 0 & 0 & 0 \\
0 & 0 & 0 & 0 & 0 & 0 \\
0 & 0 & S & S \hat{y}_{f} & -S \hat{x}_{f} & 0 \\
0 & 0 & S \hat{y}_{f} & S_{\hat{y} \hat{y}}+\mathcal{V}_{0} \hat{z}_{b} & -S_{\hat{x} \hat{y}} & \mathcal{V}_{0} \hat{x}_{b} \\
0 & 0 & -S \hat{x}_{f} & -S_{\hat{x} \hat{y}} & S_{\hat{x} \hat{x}}+\mathcal{V}_{0} \hat{z}_{b} & -\mathcal{V}_{0} \hat{y}_{b} \\
0 & 0 & 0 & \mathcal{V}_{0} \hat{x}_{b} & -\mathcal{V}_{0} \hat{y}_{b} & 0
\end{array}\right]
$$

with $S$ the waterplane area, $S_{j}$ the first waterplane moments (e.g. $\left.S_{\hat{x}}=\iint_{S} \hat{x} \mathrm{~d} S\right), S_{j l}$ the second waterplane moments (e.g. $S_{\hat{x} \hat{y}}=\iint_{S} \hat{x} \hat{y} \mathrm{~d} S$ ), and $\hat{x}_{f}$ and $\hat{y}_{f}$ the coordinates of the center of flotation, defined as $\hat{x}_{f}=S_{\hat{x}} / S$ and $\hat{y}_{f}=S_{\hat{y}} / S$. In naval architecture, the contribution due to the weight of the body is traditionally added to the hydrostatic moments, but since in here they are given with respect to the center of gravity, this contribution is zero.

The second-order hydrostatic loads are obtained by multiplying the same hydrostatic matrix and the second-order motions, $\mathcal{F}_{H S}^{(2)}=-\mathbb{K}_{H S} \boldsymbol{\xi}^{(2)}$.

Since the body is considered to be composed of cylinders only, the hydrostatic matrix can be evaluated by computing the contribution of each cylinder, which can be done analytically, and then summing them up appropriately. Considering a cylinder with the origin of the local coordinate system Oxyz located at its bottom and with a rotation of $\alpha$ with respect to the vertical, which is always around the local $y$ axis of the cylinder due to the way that $O x y z$ is defined, the quantities needed from each cylinder to evaluate $\mathbb{K}_{H S}$ can be expressed as follows:

$$
\begin{aligned}
& \mathcal{V}_{0}=\pi R^{2} L_{0} \\
& x_{b}=\frac{\pi R^{4} \tan \alpha}{4 \mathcal{V}_{0}} \quad y_{b}=0 \quad z_{b}=\frac{R^{2} \tan ^{2} \alpha+4 L_{0}^{2}}{8 L_{0}} \\
& S=\frac{\pi R^{2}}{\cos \alpha} \quad S_{x x}=\frac{1}{4} \pi R^{4} \quad S_{y y}=\frac{1}{4} \frac{\pi R^{4}}{\cos \alpha}
\end{aligned}
$$

as these quantities are given with respect to the local coordinate system of each cylinder, they need to be transformed to the body coordinate axes, $G \hat{x} \hat{y} \hat{z}$. This is quite simple for the center of buoyancy, while the necessary formulae for the moments of area can be found in França and Matsumura (2011).

The equations above consider the case of a cylinder crossing the mean waterline in such a way that one of its end faces is completely submerged and the other is completely above the water; in other words, the case where the end faces of the cylinder are crossed 
by the mean waterline (a floating horizontal cylinder, for example) is not covered. If the cylinder is completely submerged, the waterline integrals are all zero, while the determination of the submerged volume and its center is trivial.

\subsection{Total hydrodynamic forces acting on the floater}

The hydrodynamic forces and moments acting on a structure composed of $N_{c}$ cylinders are given by the sum of the different components presented in this chapter, as summarized below. They are divided into the following components:

$$
\begin{aligned}
\mathcal{F}^{(0)} & =\mathcal{F}_{H S}^{(0)} \\
\mathcal{F}^{(1)} & =-\mathbb{A} \ddot{\boldsymbol{\xi}}^{(1)}-\mathbb{K} \boldsymbol{\xi}^{(1)}+\mathcal{F}_{\phi^{(1)}}^{(1)} \\
\mathcal{F}^{(2)} & =-\mathbb{A}^{(2)}-\mathbb{K} \boldsymbol{\xi}^{(2)}+\mathcal{F}_{\text {drag }}+\mathcal{F}_{A_{T}^{(1)}}^{(2)}+\mathcal{F}_{\phi^{-}}^{(2)}+\mathcal{F}_{\text {conv }}^{(2)}+\mathcal{F}_{\text {axdv }}^{(2)}+\mathcal{F}_{\mathrm{rslb}}^{(2)} \\
& +\mathcal{F}_{\eta}^{(2)}+\mathcal{F}_{\text {rot } \mathrm{N}}^{(2)}+\mathcal{F}_{\mathbf{X} \cdot \nabla}^{(2)}+\mathcal{F}_{\text {rem }}^{(2)}
\end{aligned}
$$

in which $\mathcal{F}$ is the six-component vector of loads, with the first three elements corresponding to the forces and the remaining three to the moments with respect to the center of gravity, i.e. $\mathcal{F}=\left(F_{1} ; F_{2} ; F_{3} ; M_{G, 1} ; M_{G, 2} ; M_{G, 3}\right)$.

The terms of the equations above are the sum of the forces integrated along the length of each cylinder and the loads at both their extremities. The end of the cylinder that coincides with its bottom is identified by the affix ' $b$ ' (for bottom), while 't' (for top) is related to the opposite extremity, and the local $z$ axis is taken as positive pointing from the bottom of the cylinder to its top. It is understood that if an end of the cylinder is above the mean waterline, the associated load is zero.

Each cylinder $j$ has its own diameter $D_{j}$, local unit vectors $\left\{\mathbf{e}_{1, j}, \mathbf{e}_{2, j}, \mathbf{e}_{3, j}\right\}$, drag coefficient $C_{D, j}$, etc. However, keeping the subscript $j$ is somewhat cumbersome, so it is omitted.

Forces due to body acceleration: besides the terms $-\mathbb{A} \ddot{\boldsymbol{\xi}}^{(1)}$ and $-\mathbb{A} \ddot{\boldsymbol{\xi}}^{(2)}$, body accelerations also leads to a second-order force due to the first-order variation of the added mass matrix:

$$
\mathcal{F}_{A_{T}^{(1)}}^{(2)}=-\left(\mathbb{A}_{T}^{(1)}-\mathbb{A}\right) \ddot{\boldsymbol{\xi}}^{(1)}
$$

with the added mass matrix calculated with the formulae presented in Appendix A. The force due to the centripetal acceleration of a point of the body is included in component $\mathcal{F}_{\text {rem }}^{(2)}$ (the last to be discussed in this list).

Drag: groups the quadratic drag force along the length of the cylinder with the one 
concentrated at each cylinder end, as presented in Section 3.4.

$$
\begin{aligned}
\mathbf{F}_{\mathrm{drag}}=\sum_{L_{0}}^{N_{c}} \int_{L_{0}} \frac{1}{2} \rho D C_{D}\left\|\mathbf{u}_{P}^{(1)}-\dot{\mathbf{x}}_{P}\right\|\left(\mathbf{u}_{P}^{(1)}-\dot{\mathbf{x}}_{P}\right) \mathrm{d} z \\
\quad+\frac{1}{2} \rho \pi R^{2}\left(C_{D z, b}\left|w_{b}^{(1)}-\dot{z}\right|\left(w_{b}^{(1)}-\dot{z}\right)+C_{D z, t}\left|w_{t}^{(1)}-\dot{z}_{B}\right|\left(w_{t}^{(1)}-\dot{z}_{B}\right)\right) \mathbf{e}_{3}^{(0)}
\end{aligned}
$$

A common element in FOWTs are heave plates, which are flat disks attached to the bottom of columns to increase added mass and damping in the vertical degrees of freedom. They can be modeled as cylinders with a very short height, and the expression above can be implemented in two ways: set $C_{D z, b}$ to the desired value of $C_{D z}$, while $C_{D z, t}$ is taken as zero; or set $C_{D z, b}=C_{D z, t}=0.5 C_{D z}$. Both approaches should yield similar results provided that the thickness is indeed small.

The moments with respect to the center of gravity, $G$, associated with $\mathbf{F}_{\text {drag }}$ can be obtained by simply including the cross product with the position vector $\mathbf{r}$ in the integration along the cylinder and in the forces at its ends:

$$
\begin{aligned}
\mathbf{M}_{\mathrm{drag}}=\sum \sum_{L_{0}}^{N_{c}} & \frac{1}{2} \rho D C_{D}\left\|\mathbf{u}_{P}^{(1)}-\dot{\mathbf{x}}_{P}\right\|\left[\mathbf{r} \wedge\left(\mathbf{u}_{P}^{(1)}-\mathbf{x}_{P}\right)\right] \mathrm{d} z \\
& +\frac{1}{2} \rho \pi R^{2} C_{D z, b}\left|w_{b}^{(1)}-\dot{z}\right|\left(w_{b}^{(1)}-\dot{z}_{B}\right)\left(\mathbf{r}_{b} \wedge \mathbf{e}_{3}^{(0)}\right) \\
& +\frac{1}{2} \rho \pi R^{2} C_{D z, t}\left|w_{t}^{(1)}-\dot{z}\right|\left(w_{t}^{(1)}-\dot{z}_{B}\right)\left(\mathbf{r}_{t} \wedge \mathbf{e}_{3}^{(0)}\right)
\end{aligned}
$$

The expression of the moment will be omitted in the remaining components, as it is basically the same as the corresponding force.

Component due to the first-order incoming flow:

$$
\begin{aligned}
\mathbf{F}_{\phi^{(1)}}^{(1)}= & \sum_{L_{0}}^{N_{c}} \int_{R^{2}} \rho \pi R^{2}\left(1+C_{a}\right)\left[\left(\frac{\partial \mathbf{u}^{(1)}}{\partial t} \cdot \mathbf{e}_{1}^{(0)}\right) \mathbf{e}_{1}^{(0)}+\left(\frac{\partial \mathbf{u}^{(1)}}{\partial t} \cdot \mathbf{e}_{2}^{(0)}\right) \mathbf{e}_{2}^{(0)}\right] \mathrm{d} z \\
+ & {\left[\pi R^{2}\left(\left.\frac{\partial \varphi^{(1)}}{\partial t}\right|_{t}-\left.\frac{\partial \varphi^{(1)}}{\partial t}\right|_{b}\right)+\rho \frac{4 \pi R^{3}}{3}\left(C_{a z, b} \frac{\partial \mathbf{u}_{b}^{(1)}}{\partial t}+C_{a z, t} \frac{\partial \mathbf{u}_{t}^{(1)}}{\partial t}\right) \cdot \mathbf{e}_{3}^{(0)}\right] \mathbf{e}_{3}^{(0)} }
\end{aligned}
$$

where $C_{a z, b}$ and $C_{a z, t}$ are the axial added mass coefficients associated with each end of each cylinder, and the same observations made above regarding the axial drag coefficients are applicable to them.

Component due to the second-order wave potential: very similar to the previous 
component, with the difference that it corresponds to the second-order incoming flow.

$$
\begin{aligned}
\mathbf{F}_{\phi^{-}}^{(2)}= & \sum_{L_{0}}^{N_{c}} \int_{L_{0}} \rho \pi R^{2}\left(1+C_{a}\right)\left[\left(\frac{\partial \mathbf{u}^{-}}{\partial t} \cdot \mathbf{e}_{1}^{(0)}\right) \mathbf{e}_{1}^{(0)}+\left(\frac{\partial \mathbf{u}^{-}}{\partial t} \cdot \mathbf{e}_{2}^{(0)}\right) \mathbf{e}_{2}^{(0)}\right] \mathrm{d} z \\
& +\left[\pi R^{2}\left(\left.\frac{\partial \varphi^{-}}{\partial t}\right|_{t}-\left.\frac{\partial \varphi^{-}}{\partial t}\right|_{b}\right)+\rho \frac{4 \pi R^{3}}{3}\left(C_{a z, b} \frac{\partial \mathbf{u}_{b}^{-}}{\partial t}+C_{a z, t} \frac{\partial \mathbf{u}_{t}^{-}}{\partial t}\right) \cdot \mathbf{e}_{3}^{(0)}\right] \mathbf{e}_{3}^{(0)}
\end{aligned}
$$

in which only the low-frequency second-order part of the incoming flow is considered. It corresponds to the force component V from Pinkster (1980).

Component due to the convective acceleration:

$$
\begin{aligned}
\mathbf{F}_{\mathrm{conv}}^{(2)}= & \sum_{L_{0}}^{N_{c}} \int_{L} \rho \pi R^{2}\left(1+C_{a}\right)\left[\left(\mathbf{a}_{c}^{(2)} \cdot \mathbf{e}_{1}^{(0)}\right) \mathbf{e}_{1}^{(0)}+\left(\mathbf{a}_{c}^{(2)} \cdot \mathbf{e}_{2}^{(0)}\right) \mathbf{e}_{2}^{(0)}\right] \mathrm{d} z \\
& +\rho \frac{4 \pi R^{3}}{3}\left[\left(C_{a z, b} \mathbf{a}_{c, b}^{(2)}+C_{a z, t} \mathbf{a}_{c, t}^{(2)}\right) \cdot \mathbf{e}_{3}^{(0)}\right] \mathbf{e}_{3}^{(0)}
\end{aligned}
$$

with

$$
\mathbf{a}_{c}^{(2)}=\left(\mathbf{u}^{(1)} \cdot \nabla\right) \mathbf{u}^{(1)}
$$

Component due to the axial-divergence acceleration:

$$
\mathbf{F}_{\mathrm{axdv}}^{(2)}=\sum^{N_{c}} \int_{L_{0}} \rho \pi R^{2} C_{a} \frac{\partial w^{(1)}}{\partial z^{(0)}}\left\{\left[\left(\mathbf{u}^{(1)}-\dot{\mathbf{x}}^{(1)}\right) \cdot \mathbf{e}_{1}^{(0)}\right] \mathbf{e}_{1}^{(0)}+\left[\left(\mathbf{u}^{(1)}-\dot{\mathbf{x}}^{(1)}\right) \cdot \mathbf{e}_{2}^{(0)}\right] \mathbf{e}_{2}^{(0)}\right\} \mathrm{d} z
$$

with $\partial w^{(1)} / \partial z^{(0)}$ given by:

$$
\frac{\partial w^{(1)}}{\partial z^{(0)}}=\left[\left(\frac{\partial \mathbf{u}^{(1)}}{\partial X} \cdot \mathbf{e}_{3}^{(0)}\right) \mathbf{E}_{1}+\left(\frac{\partial \mathbf{u}^{(1)}}{\partial Y} \cdot \mathbf{e}_{3}^{(0)}\right) \mathbf{E}_{2}+\left(\frac{\partial \mathbf{u}^{(1)}}{\partial Z} \cdot \mathbf{e}_{3}^{(0)}\right) \mathbf{E}_{3}\right] \cdot \mathbf{e}_{3}^{(0)}
$$

\section{Component due to the acceleration induced by body rotation:}

$$
\mathbf{F}_{\mathrm{rslb}}^{(2)}=\sum^{N_{c}} \int_{L_{0}}-2 \rho \pi R^{2} C_{a}\left[\left(\mathbf{u}^{(1)}-\dot{\mathbf{x}}^{(1)}\right) \cdot \mathbf{e}_{3}^{(0)}\right]\left[\left(\dot{\boldsymbol{\xi}}_{R}^{(1)} \cdot \mathbf{e}_{2}^{(0)}\right) \mathbf{e}_{1}^{(0)}-\left(\dot{\boldsymbol{\xi}}_{R}^{(1)} \cdot \mathbf{e}_{1}^{(0)}\right) \mathbf{e}_{2}^{(0)}\right] \mathrm{d} z
$$

where the affix "rslb" stands for "rotation slender body" in order to distinguish this force from the one due to the rotation of the normal vector.

Component due to the wave elevation:

$$
\begin{aligned}
\mathbf{F}_{\eta}^{(2)}=\sum^{N_{c}} \rho \pi R^{2} \eta_{r}^{(1)} & {\left[\left(\left(1+C_{a}\right) \frac{\partial \mathbf{u}^{(1)}}{\partial t} \cdot \mathbf{e}_{1}^{(0)}-\mathbf{g} \cdot \mathbf{e}_{1}^{(1)}\right) \mathbf{e}_{1}^{(0)}\right.} \\
+ & \left.\left(\left(1+C_{a}\right) \frac{\partial \mathbf{u}^{(1)}}{\partial t} \cdot \mathbf{e}_{2}^{(0)}-\mathbf{g} \cdot \mathbf{e}_{2}^{(1)}\right) \mathbf{e}_{2}^{(0)}\right]
\end{aligned}
$$


with $\eta_{r}^{(1)}=\eta_{I}^{(1)}-Z_{I}^{(1)}$ the first-order relative wave elevation evaluated at the cylinder axis.

Component due to the rotation of the normal vector:

$$
\mathbf{F}_{\mathrm{rotN}}^{(2)}=\sum^{N_{c}} \boldsymbol{\xi}_{R}^{(1)} \wedge\left(\mathbf{F}_{\phi^{(1)}}^{(1)}+\mathbf{F}_{\mathrm{HS}}^{(1)}\right)
$$

Component due to body motions inside the pressure field:

$$
\begin{aligned}
\mathbf{F}_{\mathbf{X} \cdot \nabla}^{(2)} & =\sum_{L_{0}}^{N_{c}} \rho \pi R^{2}\left(1+C_{a}\right)\left[\left(\mathbf{a}_{\mathbf{X} \cdot \nabla}^{(2)} \cdot \mathbf{e}_{1}^{(0)}\right) \mathbf{e}_{1}^{(0)}+\left(\mathbf{a}_{\mathbf{X} \cdot \nabla}^{(2)} \cdot \mathbf{e}_{2}^{(0)}\right) \mathbf{e}_{2}^{(0)}\right] \mathrm{d} z \\
& +\left\{\left[\pi R^{2}\left(\nabla p_{b}^{(1)} \cdot \mathbf{X}_{b}^{(1)}-\nabla p_{t}^{(1)} \mathbf{X}_{t}^{(1)}\right)+\rho \frac{4 \pi R^{3}}{3}\left(C_{a z, b} \mathbf{a}_{\mathbf{X} \cdot \nabla, b}^{(2)}+C_{a z, t} \mathbf{a}_{\mathbf{X} \cdot \nabla, t}^{(2)}\right)\right] \cdot \mathbf{e}_{3}^{(0)}\right\} \mathbf{e}_{3}^{(0)}
\end{aligned}
$$

with

$$
\mathbf{a}_{\mathbf{X} \cdot \nabla}^{(2)}=\nabla \frac{\partial \mathbf{u}^{(1)}}{\partial t} \cdot \mathbf{X}^{(1)}
$$

and $p_{b}^{(1)}$ and $p_{t}^{(1)}$ the first-order dynamic pressure evaluated at the bottom and top extremities of the cylinder.

Remaining force components: during the test cases analyzed in this thesis, some force terms that could not be grouped with others have been found to be negligible, namely the force due to the centripetal acceleration, the perpendicular point load at the immersed ends of the cylinder and the quadratic pressure drop from Rainey's formulation. As the number of force components is already quite large, these remaining terms are grouped in a single component $\mathbf{F}_{\text {rem }}^{(2)}$, given by:

$$
\begin{aligned}
\mathbf{F}_{\mathrm{rem}}^{(2)}=\sum^{N_{c}} & -\int_{L_{0}} \rho \pi R^{2} C_{a}\left[\left(\ddot{\mathbf{x}}_{\mathrm{ctp}}^{(1)} \cdot \mathbf{e}_{1}^{(0)}\right) \mathbf{e}_{1}^{(0)}+\left(\ddot{\mathbf{x}}_{\mathrm{ctp}}^{(1)} \cdot \mathbf{e}_{2}^{(0)}\right) \mathbf{e}_{2}^{(0)}\right] \mathrm{d} L \\
& -\rho \frac{4 \pi R^{3}}{3}\left[\left(C_{a z, b} \ddot{\mathbf{x}}_{\mathrm{ctp}, b}^{(1)}+C_{a z, t} \ddot{\mathbf{x}}_{\mathrm{ctp}, t}^{(1)}\right) \cdot \mathbf{e}_{3}^{(0)}\right] \mathbf{e}_{3}^{(0)} \\
& +C_{a} \rho \pi R^{2}\left(w_{b}^{(1)}-\dot{z}_{b}^{(1)}\right)\left[\left(u_{b}^{(1)}-\dot{x}_{b}^{(1)}\right) \mathbf{e}_{1}^{(0)}+\left(v_{b}^{(1)}-\dot{y}_{b}^{(1)}\right) \mathbf{e}_{2}^{(0)}\right] \\
& -C_{a} \rho \pi R^{2}\left(w_{t}^{(1)}-\dot{z}_{t}^{(1)}\right)\left[\left(u_{t}^{(1)}-\dot{x}_{t}^{(1)}\right) \mathbf{e}_{1}^{(0)}+\left(v_{t}^{(1)}-\dot{y}_{t}^{(1)}\right) \mathbf{e}_{2}^{(0)}\right] \\
& -\frac{1}{2} \rho \pi R^{2} C_{a}\left[\left(u_{b}^{(1)}-\dot{x}_{b}^{(1)}\right)^{2}+\left(v_{b}^{(1)}-\dot{y}_{b}^{(1)}\right)^{2}-\left(u_{t}^{(1)}-\dot{x}_{t}^{(1)}\right)^{2}+\left(v_{t}^{(1)}-\dot{y}_{t}^{(1)}\right)^{2}\right] \mathbf{e}_{3}^{(0)}
\end{aligned}
$$

which, based on the test cases, could be discarded with no significant loss to the results. Nevertheless, it is possible that there may be situations where this force component is important.

Most of the integrals presented in this chapter are evaluated numerically, but the forces $\mathbf{F}_{\phi^{(1)}}^{(1)}$ and $\mathbf{F}_{\phi^{(2)}}^{(2)}$ and their respective moments have analytical solution when considering the wave potentials given by Equations 3.33 and 3.35, as described in Appendix B. 


\subsection{The choice of $C_{a}$ and $C_{D}$}

Though they look simple, the real difficulty with a slender-body method such as Morison's equation or any other that uses the empirical coefficients of added mass and drag lies in the proper determination of these coefficients. As pointed out by Sarpkaya (2001, 2010), since the kinetic velocity of the flow is affected by the flow separation, even the added mass coefficient $C_{a}$ needs to be determined, as it is different from the one calculated considering potential flow. These coefficients are dependent on several parameters that describe the flow, such as the geometry of the body, surface roughness, Keulegan-Carpenter number $K C$, and Reynolds number $R e$. This is a major complication, as each analysis requires $C_{a}$ and $C_{D}$ to be chosen somehow, normally from experimental tests. Due to the resonant nature of the slow-drift motions of floating structures, proper determination of the drag coefficient is of paramount importance, as it directly impacts the damping levels and, consequently, the amplitude of the slow-drift motion, while the added mass coefficient can have a large influence on the natural frequencies.

A further complication lies in the fact that these coefficients are actually time dependent quantities that depend both on the instantaneous kinematics of the flow and on its history. For a periodic motion such as the one experienced by a floating structure under the action of regular waves, an approach that works well is to use Fourier averaged coefficients (KEULEGAN; CARPENTER, 1958; SARPKAYA, 2001), but this is not necessarily true when the incoming flow is composed of more than one frequency, as is the case for a real sea, and when body motions are a combination of periodic components with very different frequencies, for instance the superposition of wave-frequency and slow-drift motions of a moored FOWT. This is an important topic in current research regarding floating wind turbines, as exemplified by the OC5 project (ROBERTSON; WENDT, et al., 2017), in which one of the hypothesis to explain the large discrepancy observed between numerical and experimental results regarding low-frequency motions is that the drag coefficients were overestimated in the numerical analyses, and this issue is currently under investigation in the OC6 project.

In one of the works that was developed during this thesis (CARMO; MELLO, et al., 2020), two possible methods to estimate the coefficients, namely decay tests and forced oscillations (both in still water), were analyzed in order to verify whether proper values of force coefficients could be obtained. The results were verified by comparing experimental slow surge motions of a FOWT under the action of bichromatic waves with the ones calculated using WAMIT considering two different external damping values: one calibrated from the decay tests and the other from drag coefficients measured in the forced oscillations. The damping levels obtained with the latter were lower than the ones evaluated from the decay tests, resulting in larger motions in the corresponding numerical simulations. Bearing in mind all the simplifications involved and the uncertainties related to the experimental 
tests, the agreement between numerical simulations and the experiment was very good, with better results using the damping values from forced oscillations, though a general trend of under predicting the slow-drift motions was observed. This test campaign is better detailed in Chapter 6, as it was an earlier phase of the experiments presented therein. Since the same floater was tested in both phases, the results of the same forced oscillations are used to calibrate the added mass and drag coefficients of the numerical simulations discussed in that chapter.

Besides the problem of tuning the hydrodynamic coefficients, it would also be possible to change the formulation of the drag force. For instance, while the original quadratic drag term employed in Morison's equation considers the total instantaneous relative velocity between the fluid and the body, it would be possible to separate the velocity due to the slow-drift motion from the one at the frequency of the waves, associating different drag coefficients to each of them (MOLIN, 1993; EMMERHOFF; SCLAVOUNOS, 1996). This so-called 'independent' form of Morison's equation allows the separation of the wave and low-frequency viscous loads, but Molin (1993) points out that there are no physical evidences to choose between these two versions of the drag force. Based on experimental results, he concludes that neither is strictly correct, but also points out that the traditional form of Morison's equation (called 'dependent' in his work) can overestimate the lowfrequency damping. Investigating different drag formulations is not among the objectives of this thesis, as the focus is on the inertial part of the hydrodynamic forces, but it would be an interesting continuation to improve the slender-body approximation and even simulations where quadratic drag is added to the forces calculated with radiation/diffraction solvers. 


\section{Numerical method}

This chapter describes the numerical implementation of METiS (Morison Equation Time Domain Simulation), a numerical tool for the analysis of floating offshore wind turbines. More accurately, it describes the first steps of the development of the software, which is intended to be continued in the future with the contribution of other members of the research group in order to make it a comprehensive tool. At the current stage, it is capable of evaluating the first- and second-order wave loads on the floater using the slender-body approximation presented in this thesis and the aerodynamic loads on the rotor with Blade Element Momentum Theory (BEMT), which are then used to solve the equations of motion in time domain.

It starts by discussing the numerical evaluation of the hydrodynamic forces discussed in Chapter 3, followed by a brief explanation of BEMT and the inclusion of other forces relevant to this text, namely a simple linear stiffness matrix to model the restoring forces imposed by the mooring system and a constant linear damping matrix for the cases where wave radiation damping is relevant. Then, the equations of motion of the FOWT, which is considered to be a single rigid body, are presented along with the numerical scheme used to solve them (a standard $4^{\text {th }}$ order Runge-Kutta method). Finally, the overall structure of the software is summarized, and the methodology used to post process the results in the remaining chapters is detailed.

The software was written in $\mathrm{C}++$ using Armadillo (SANDERSON; CURTIN, 2016) and Intel ${ }^{\circledR}$ Math Kernel Library (WANG et al., 2014).

\subsection{Evaluation of the hydrodynamic loads}

The hydrodynamic forces given in Chapter 3 involve the evaluation of wave kinematic properties at the extremities of the cylinder and along its length, with the latter requiring these quantities to be integrated. The contributions of the first- and second-order fluid acceleration, $\mathbf{F}_{\phi^{(1)}}^{(1)}$ and $\mathbf{F}_{\phi^{-}}^{(2)}$, are integrated analytically in Appendix B, but the others are integrated numerically using Simpson's rule:

$$
\int_{0}^{L} f(z) \mathrm{d} z \approx \frac{\Delta z}{3}\left[f(0)+2 \sum_{j=1}^{n / 2-1} f\left(z_{2 j}\right)+4 \sum_{j=1}^{n / 2} f\left(z_{2 j-1}\right)+f(L)\right]
$$

with $z$ following the axis of the cylinder with origin at its bottom (like in Chapter 3) and $n$ the number of sub-divisions of the cylinder, which needs to be an even number.

The functions that need to be numerically integrated are all products of first-order quantities. The ones related to body motions are obtained at each time step as a result of 
the equations of motion, while an arbitrary first-order wave kinematic quantity $Q^{(1)}(t)$, given by:

$$
Q^{(1)}(t)=\Re\left\{\sum_{j=0}^{N} q_{j}^{(1)} e^{-i \omega_{j} t}\right\}
$$

with $N$ the number of wave components, can be readily evaluated using an Inverse Fast Fourier Transform (IFFT) algorithm, i.e.:

$$
Q^{(1)}(t)=\Re\left\{\operatorname{IFFT}\left(q_{j}^{(1)}\right)\right\}
$$

As mentioned before, this is the reason why the slender-body approximation proposed by Madsen (1986), Rainey (1989), and Manners (1992) was modified in order to allow the evaluation of the wave loads considering the fixed body position, since the whole time series of wave kinematics can be determined very efficiently at the beginning of the simulation. Indeed, in order to avoid signal repetition, an irregular wave simulation with time step $\Delta t$ and total simulation time $T_{\text {Total }}=N \Delta t$ requires a frequency resolution $\Delta \omega=2 \pi /(N \Delta t)$ (assuming that $\Delta \omega_{j}=\omega_{j+1}-\omega_{j}$ is constant). Hence, in order to directly sum the contribution of each wave component, a number of frequency components of $\mathcal{O}(N)$ would be necessary to cover the whole range of the wave spectrum, leading to a computational complexity of $\mathcal{O}\left(N^{2}\right)$ because this operation would need to be performed at each time step. Meanwhile, performing an IFFT of the signal has a complexity of $\mathcal{O}\left(N \log _{2} N\right)$ (PRESS et al., 1992), which is a huge difference. For example, the $3 \mathrm{~h}$ irregular sea simulations presented in Chapter 6 required $N \approx 10^{5}$, meaning that the IFFT is roughly 6000 faster than the direct summation.

The force due to the second-order potential, $\mathbf{F}_{\phi^{-}}^{(2)}$, is a little trickier. While the other second-order forces are due to products of first-order quantities, this one requires the evaluation of the following double sum:

$$
\mathbf{F}_{\phi^{-}}^{(2)}=\Re\left\{\sum_{j=0}^{N} \sum_{l=0}^{N} \mathbf{f}_{\phi^{-}, j l}^{(2)} e^{-i\left(\omega_{j}-\omega_{l}\right) t}\right\}
$$

but this is so computationally inefficient that even moderate values of $N$ become prohibitive, as the cost would be of $\mathcal{O}\left(N^{3}\right)$. However, as detailed by Agarwal and Manuel (2011), it is possible to rewrite the double summation as a single summation by grouping the complex amplitudes $\mathbf{f}_{\phi^{-}, j l}^{(2)}$ :

$$
\hat{\mathbf{f}}_{\phi^{-}, k}^{(2)}=\sum_{j=0}^{N} \sum_{l=j+k} \mathbf{f}_{\phi^{-}, j l}^{(2)}+\mathbf{f}_{\phi^{-}, l j}^{(2) *}
$$

which, following the usual convention of representing second-order quantities as matrices, is equivalent to summing the coefficients of each diagonal. After this procedure of rearranging terms, the force $\mathbf{F}_{\phi^{-}}^{(2)}$ can be rewritten as a single sum:

$$
\mathbf{F}_{\phi^{-}}^{(2)}=\Re\left\{\sum_{j=0}^{N} \hat{\mathbf{f}}_{\phi^{-}, j}^{(2)} e^{-i \Delta \omega_{j} t}\right\}
$$


with $\Delta \omega_{j}=j \Delta \omega$. This expression can be evaluated using an IFFT algorithm, thus the force due to the second-order potential can be calculated with the same cost of the firstorder quantities after the reorganization of the coefficients given by Equation 4.5. This reorganization, however, represents a computational cost of $\mathcal{O}\left(N^{2}\right)$, which is still somewhat costly. Nevertheless, it represents a reduction of $\mathcal{O}(N)$ from the direct sum, which is very significant.

\subsection{Other forces acting on the structure}

\subsubsection{Aerodynamic forces on the rotor}

The aerodynamic loads, $\mathcal{F}_{\text {aero }}$, are computed using Blade Element Momentum Theory (BEMT), a very common method to evaluate aerodynamic loads on the rotor of a wind turbine. Its widespread use is due to its simplicity and capability of providing fast and good results. It is implemented in METiS based on the numerical code developed by Pegoraro (2018), where details concerning the theoretical background of BEMT and its implementation can be found.

In short, the method combines momentum theory and blade element theory to evaluate the forces per unit length acting along the blades, which are integrated to obtain the total loads acting on the rotor. Besides the characterization of the geometry of the blades (chord, twist angle, etc), it requires the lift, drag and moment coefficients of each section of the blade as a function of the angle of attack. The core of the method is to determine the so called axial and tangential induction factors for each of these sections, assumed to be independent from the others. These factors measure the perturbation of the incoming wind flow when it approaches the rotor, and are sufficient to calculate the local inflow angle (and, by consequence, the local angle of attack) and velocity. With the angle of attack and the local inflow velocity, which are evaluated taking into account body motions, the forces and moments acting on each section of the blades can be determined using the known lift, drag and moment coefficients. Except for a few elementary cases, the evaluation of the induction factors needs to be performed numerically, which in METiS is done using the procedure proposed by Ning (2014).

The incoming wind flow is considered horizontal and constant in time, but height dependent following a power law profile:

$$
U_{w}(Z)=U_{Z_{r}}\left(\frac{Z}{Z_{r}}\right)^{p}
$$

where $U_{Z_{r}}$ is the wind speed at the reference height, $Z_{r}$, and $p$ is the power law exponent.

As body motions are considered in the aerodynamic calculation, the aerodynamic forces are a function of the displacement and velocity of the FOWT. If control of the 
blades was included in the model, the aerodynamic loads would also be a function of body acceleration in the above-rated regime (SOUZA; BACHYNSKI, 2019), but this is not the case here.

\subsubsection{External hydrodynamic damping}

A known drawback of a slender-body approach such as Morison's equation is that the quadratic drag alone is not sufficient to properly reproduce the damping levels in some cases where damping due to wave radiation is relevant. For instance, one of the analyses presented in Chapter 6 concerns a FOWT under the action of bichromatic waves with small amplitudes, which has shown to be a situation where neglecting radiation damping leads to a poor estimation of the vertical motions around their natural frequencies. This is specially troublesome in this work because it makes it hard to tell whether occasional differences in the comparisons of second-order forces/motions presented in the following chapters, both with other software and experiments, are due to the force model developed in this thesis or simply a consequence of getting the first-order motions wrong. As an workaround, a frequency-independent external linear damping matrix is used:

$$
\mathcal{F}_{B_{\text {ext }}}=-\mathbb{B}_{\text {ext }} \dot{\boldsymbol{\xi}}
$$

with the terms of $\mathbb{B}_{\text {ext }}$ set to match the radiation damping at the resonance frequency of each degree of freedom, which can be calculated using, for example, WAMIT (WAMIT, 2004) or other similar software.

\subsubsection{Mooring forces}

One important subsystem of FOWTs are the moorings, which are used to keep the excursions of the structure within reasonable limits. For doing so, it induces forces and moments that act on the sense of restoring the structure to its equilibrium position. There is a myriad of approaches for modeling the mooring system with varying levels of complexity and fidelity, and this work uses the simplest of them, which is to consider a linearized mooring model consisting of a linear stiffness matrix, $\mathbb{K}_{M}$, and a constant force/moment, $\mathcal{F}_{M}$, to account for the weight of the moorings:

$$
\mathcal{F}_{M}=-\mathbb{K}_{M} \boldsymbol{\xi}+\overline{\mathcal{F}}_{M}
$$

which requires the values of $\mathbb{K}_{M}$ and $\overline{\mathcal{F}}_{M}$ to be known beforehand, using, for example, the formulation proposed by Pesce, Amaral, and Franzini (2018) and Amaral (2020). Although the linear model is considered to be sufficient for this work, as the focus is on the hydrodynamic model, improving the modeling of the mooring system is one of the main steps for the future development of the software, not only for a more accurate calculation of the motions of the FOWT, but also because the analysis of the mooring dynamics itself is a particularly important application of a numerical tool such as the one developed here. 


\subsection{Solving the equations of motion}

A floating offshore wind turbine consists of different bodies with relative motions among them. For instance, the nacelle, which is able to yaw around the tower, or the hub, which rotates around the low-speed shaft, are separate bodies from the floater. These relative motions induce important effects in the dynamics of the FOWT, such as the gyroscopic yaw moment induced by the combination of rotor rotation and pitch motion (JONKMAN, 2007).

Although the effects induced by these relative motions are important, they are not modeled in the numerical method yet, and including them is one of the next steps of the software development. At present, the whole system is considered to be a rigid body. In this case, the equations of motion are well known (MA; PATEL, 2001):

$$
\begin{gathered}
m \ddot{\boldsymbol{\xi}}_{T}=\mathbf{F} \\
\mathbb{I}_{G} \frac{\mathrm{d} \boldsymbol{\Omega}}{\mathrm{d} t}+\boldsymbol{\Omega} \wedge \mathbb{I}_{G} \boldsymbol{\Omega}=\mathbf{M}_{G}
\end{gathered}
$$

where $\mathbf{F}$ and $\mathbf{M}_{G}$ are the total forces and moments acting on the structure, $\mathbb{I}_{G}$ is the inertia matrix around the center of gravity and $\Omega$ is related to the rotation $\boldsymbol{\xi}_{R}$ by:

$$
\mathbb{T} \frac{\mathrm{d} \boldsymbol{\xi}_{R}}{\mathrm{~d} t}=\Omega
$$

with $\mathbb{T}$ a matrix formed by Euler angles following a rotation order of roll, pitch and yaw:

$$
\mathbb{T}=\left[\begin{array}{ccc}
\cos \xi_{5} \cos \xi_{6} & \sin \xi_{6} & 0 \\
-\cos \xi_{5} \sin \xi_{6} & \cos \xi_{6} & 0 \\
\sin \xi_{5} & 0 & 1
\end{array}\right]
$$

As the computation of the second-order wave forces requires the first-order motions to be obtained in advance, the equations above are applied in two steps. First, the firstorder motions are solved considering only the linear terms of the equations of motion, i.e. the term $\boldsymbol{\Omega}^{(1)} \wedge \mathbb{I}_{G} \boldsymbol{\Omega}^{(1)}$ is disregarded for being of second-order and the rotation velocity is $\Omega^{(1)}=\dot{\boldsymbol{\xi}}_{R}^{(1)}$, resulting in the following equations for the first-order motions:

$$
(\mathbb{M}+\mathbb{A}) \ddot{\boldsymbol{\xi}}^{(1)}=-\mathbb{B}_{\text {ext }} \dot{\boldsymbol{\xi}}^{(1)}-\left(\mathbb{K}_{H S}+\mathbb{K}_{M}\right) \boldsymbol{\xi}^{(1)}+\mathcal{F}_{\text {drag }}\left(\dot{\boldsymbol{\xi}}^{(1)}\right)+\mathcal{F}_{\text {aero }}\left(\boldsymbol{\xi}^{(1)}, \dot{\boldsymbol{\xi}}^{(1)}\right)+\mathcal{F}_{\phi^{(1)}}^{(1)}
$$

where the forces are the ones presented in the previous sections. Even though the aerodynamic and drag forces are not linear, they affect the first-order motions. Instead of linearizing them as usually done to include these forces in frequency-domain analyzes, the approach that was followed in this work was simply to incorporate them in the solution of the first-order problem, but considering $\dot{\boldsymbol{\xi}}^{(1)}$ and $\boldsymbol{\xi}^{(1)}$ in their evaluation. The matrix $\mathbb{M}$ is obtained by assembling the mass of the body $m$ and the inertia matrix around the center 
of gravity $\mathbb{I}_{G}$ :

$$
\mathbb{M}=\left[\begin{array}{cccccc}
m & 0 & 0 & 0 & 0 & 0 \\
0 & m & 0 & 0 & 0 & 0 \\
0 & 0 & m & 0 & 0 & 0 \\
0 & 0 & 0 & I_{x x} & I_{x y} & I_{x z} \\
0 & 0 & 0 & I_{x y} & I_{y y} & I_{y z} \\
0 & 0 & 0 & I_{x z} & I_{y z} & I_{z z}
\end{array}\right]
$$

After solving the first-order motions, the second-order forces can be evaluated. But instead of solving the second-order motions separately, the approach followed in this work is to sum all the forces and calculate the total motions of the body. For doing so, the equations of motion are written as follows:

$$
\begin{aligned}
(\mathbb{M}+\mathbb{A}) \frac{\mathrm{d} \mathbf{Q}}{\mathrm{d} t} & =-\mathbb{B}_{\text {ext }} \dot{\boldsymbol{\xi}}-\left(\mathbb{K}+\mathbb{K}_{M}\right) \boldsymbol{\xi}+\mathcal{F}_{\text {aero }}+\mathcal{F}_{\text {drag }}+\mathcal{F}_{\phi^{(1)}}^{(1)}+\mathcal{F}_{A_{t}^{(1)}}^{(2)}+\mathcal{F}_{\phi^{-}}^{(2)}+\mathcal{F}_{\text {conv }}^{(2)} \\
& +\mathcal{F}_{\text {axdv }}^{(2)}+\mathcal{F}_{\mathrm{rslb}}^{(2)}+\mathcal{F}_{\eta}^{(2)}+\mathcal{F}_{\text {rotN }}^{(2)}+\mathcal{F}_{\mathbf{X} \cdot \nabla}^{(2)}+\mathcal{F}_{\text {rem }}^{(2)}-\left[\begin{array}{c}
0 \\
\mathbf{0} \\
\dot{\boldsymbol{\xi}}_{R}^{(1)} \wedge \mathbb{I}_{G} \dot{\boldsymbol{\xi}}_{R}^{(1)}
\end{array}\right] \\
\frac{\mathrm{d}}{\mathrm{d} t}\left[\begin{array}{c}
\boldsymbol{\xi}_{T} \\
\mathbb{T} \boldsymbol{\xi}_{R}
\end{array}\right] & =\mathbf{Q}
\end{aligned}
$$

where the drag force is neither of first nor second-order because of the utilization of the total body velocity, which introduces higher order terms. Likewise, the aerodynamic forces, which are calculated considering the instantaneous body position and velocity, can not be classified as first or second-order as well.

Equation 4.14 can be organized as a set of coupled first-order ordinary differential equations (ODEs), just like done for Equations 4.16 and 4.17, following the general form $\dot{\mathbf{q}}=\mathbf{f}(t, \mathbf{q})$. There is a large number of methods to numerically integrate a set of ODEs, and a $4^{\text {th }}$ order Runge-Kutta method (RK4) (PRESS et al., 1992) with fixed step is chosen in this work due to its simplicity. The solution is given by:

$$
\begin{aligned}
\mathbf{q}(t+\Delta t) & =\mathbf{q}(t)+\frac{1}{6}\left(\mathbf{k}_{1}+2 \mathbf{k}_{2}+2 \mathbf{k}_{3}+\mathbf{k}_{4}\right)+\mathcal{O}\left(\Delta t^{5}\right) \\
\mathbf{k}_{1} & =\Delta t \mathbf{f}(t, \mathbf{q}(t)) \\
\mathbf{k}_{2} & =\Delta t \mathbf{f}\left(t+\frac{\Delta t}{2}, \mathbf{q}(t)+\frac{\mathbf{k}_{1}}{2}\right) \\
\mathbf{k}_{3} & =\Delta t \mathbf{f}\left(t+\frac{\Delta t}{2}, \mathbf{q}(t)+\frac{\mathbf{k}_{2}}{2}\right) \\
\mathbf{k}_{4} & =\Delta t \mathbf{f}\left(t+\Delta t, \mathbf{q}(t)+\mathbf{k}_{3}\right)
\end{aligned}
$$

In order to avoid impulsive responses, the incident wave amplitude and wind velocity are multiplied by a time ramp $r(t)$, so that the forces acting on the structure increase 
smoothly instead of starting with a sudden large value:

$$
r(t)= \begin{cases}\frac{1}{2}\left[1-\cos \left(\frac{\pi t}{T_{r}}\right)\right] & \text { if } t \leq T_{r} \\ 1 & \text { if } t>T_{r}\end{cases}
$$

\subsection{Outline of the numerical method}

The present version of the numerical method consists of the following steps:

1. Reading of the input data: the data concerning the FOWT (e.g. inertia matrix, properties of the cylinders that compose the floater such as $C_{a}$ and $C_{D}$, characterization of the blades, moorings, among others), the environmental conditions (acceleration of gravity, characterization of the waves, wind speed, etc) and numerical parameters (time step, total simulation time, etc) are provided to the software by an input text file, as exemplified in Appendix C. The input is verified to guarantee that all the required data was provided and whether they are valid;

2. Calculation of the added mass and hydrostatic stiffness matrices: based on the position of each extremity of the cylinders, the added mass matrix $\mathbb{A}$ is computed using the equations presented in Appendix A, while the hydrostatic stiffness matrix $\mathbb{K}$ is calculated with the equations from Section 3.5;

3. Evaluation of wave kinematics: the amplitudes of the waves that compose the incoming sea are used to compute the whole time series of wave kinematics at points of interest with IFFT. These points are nodes along the length of the cylinder, which are used to integrate the forces numerically, or selected locations at the fluid domain (e.g. a point on the free surface that acts as a wave probe). The time series of $\mathcal{F}_{\phi^{(1)}}^{(1)}$ and $\mathcal{F}_{\phi^{(-)}}^{(2)} \mathrm{m}$ which are integrated analytically, are also computed;

4. Calculation of the first-order motions: the forces from Equation 4.14 are evaluated based on first-order wave kinematics and the FOWT position $\boldsymbol{\xi}_{n-1}^{(1)}$ and velocity $\dot{\boldsymbol{\xi}}_{n-1}^{(1)}$ obtained in the previous time step. They are then used to calculate the first-order motions using the RK4 method;

5. Calculation of the total motions: the forces from Equation 4.16 are evaluated based on wave kinematics, the FOWT position $\boldsymbol{\xi}_{n-1}$ and velocity $\dot{\boldsymbol{\xi}}_{n-1}$ obtained in the previous time step, and the first-order acceleration $\ddot{\boldsymbol{\xi}}_{n}^{(1)}$ computed in the current time step. They are then used to calculate the total motions using the RK4 method.

The simulation ends when the total simulation time is achieved. In order to save memory and avoid complete loss of data in possible crashes, the results of the simulation 
are written to an output file at the end of each time step. The numerical method is outlined in Figure 8.

Figure 8 - Flowchart with the main steps of the numerical method.

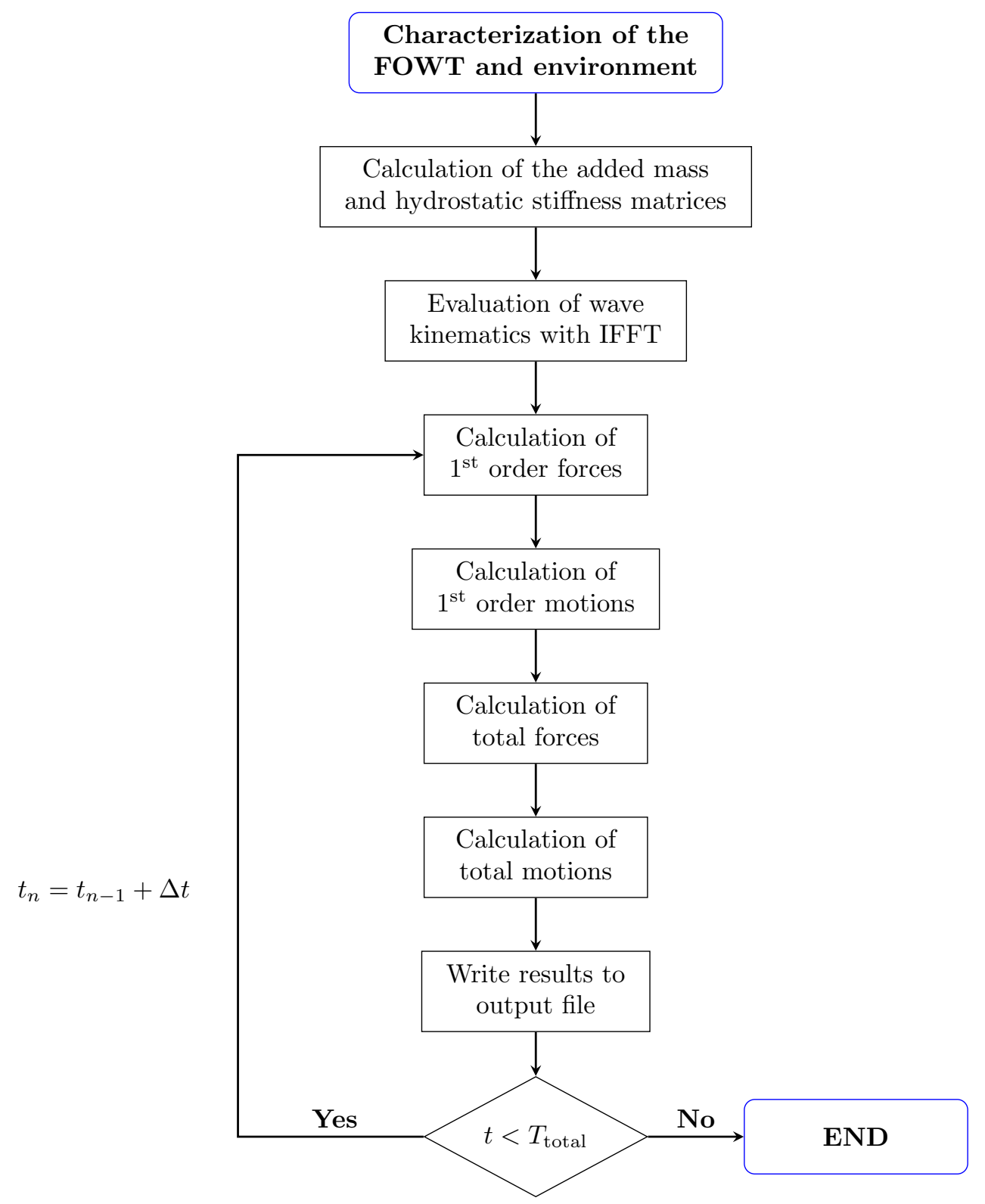




\section{Low-frequency wave forces on a surface- piercing cylinder}

One of the most fundamental applications of the method presented in this thesis is the analysis of a surface-piercing cylinder, be it bottom mounted or floating. Due to its simplicity, this particular case allows a detailed examination of the different hydrodynamic force components, leading to interesting conclusions about the physics of the slender-body approximation.

The first part of this chapter is about the difference-frequency second-order wave forces acting on a bottom mounted cylinder, a classic hydrodynamic problem with analytical solution for both diffraction theory (KIM; YUE, 1990) and the slender-body approximation, and it is centered around comparisons of the forces due to bichromatic waves for different values of the dimensionless parameters that characterize the problem. For simplicity, the analysis is restricted to long-crested waves. Since the objective is to evaluate the importance of potential flow effects such as wave diffraction, the quadratic drag term from the slender-body formulation is not considered; besides, as extensive comparisons of Morison's equation and diffraction theory already exist in the literature (see, for instance, Hooft (1972)), first-order wave forces are kept out of the analysis. It is shown that the slender-body approximation predicts very well the imaginary part of the second-order force, but it fails to capture its real part, which seems to be related to diffraction effects. On the other hand, Newman's approximation shows an opposite behavior, modeling only the real part of the force, indicating that it may be possible to combine both approximations without accounting for the same effects twice. Though these conclusions have already been noted by Molin and Chen (2002), the analysis is expanded here to include comparisons on the different components that constitute the force and to relate them to the incident and diffracted waves.

The complexity of the test case is increased in the second part of the chapter, in which two different floating cylinders are analyzed in order to assess the impact of the first-order motions on the second-order loads. Since this case does not have an analytical solution, the solution with the slender-body approximation is obtained with METiS, while WAMIT is used to solve the problem with radiation/diffraction theory. In opposition to the fixed case, the second-order force calculated with METiS has a non-zero real part, in such a way that the complementarity between Newman's and the slender-body approximation is not as easy to be exploited.

Both test cases show the relevance of the convective acceleration, axial-divergence and rotation terms from Rainey's formulation, evidencing that the common practice of 
analyzing second-order loads by simply applying Morison's equation with second-order wave kinematics is not strictly correct, as these additional terms should not be disregarded before verifying their impact on the results.

\subsection{Bottom mounted vertical cylinder}

The problem at hand, which is illustrated in Figure 9, is to evaluate the low-frequency second-order wave forces and moments acting on a fixed vertical cylinder with constant diameter extending from the sea bottom and crossing the mean water surface, with enough freeboard so that the extremities of the cylinder are unimportant.

Figure 9 - Illustration of the bottom mounted vertical cylinder under the action of a bichromatic wave.

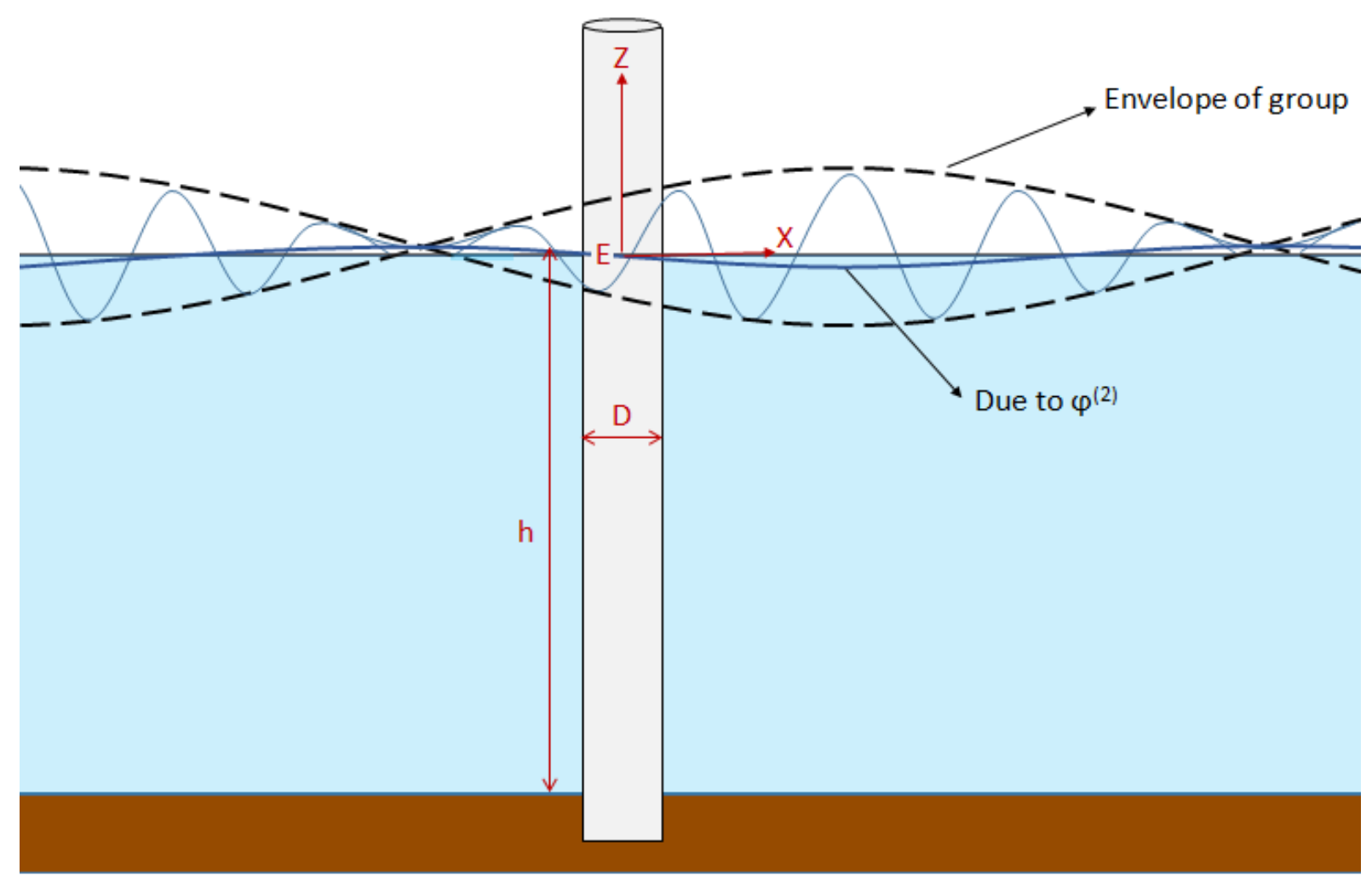

Assuming potential flow and long crested waves, the nondimensional forces can be completely described as functions of the following dimensionless parameters:

$$
\bar{k}_{j}=k_{j} R \quad \bar{h}=\frac{h}{R} \quad \bar{\omega}_{j l}^{-}=\frac{\omega_{j}-\omega_{l}}{\sqrt{g / h}}
$$

with $R$ being the cylinder radius, $k_{j}$ and $\omega_{j}$ the wave number and angular frequency associated with the $j^{\text {th }}$ wave component, $h$ the water depth and $g$ the acceleration of gravity. All the other relevant dimensionless quantities can be obtained from these three, for example:

$$
k_{j} h=\bar{k}_{j} \bar{h}
$$




$$
\begin{gathered}
\sqrt{\bar{k}_{l} \bar{h} \tanh \bar{k}_{l} \bar{h}}=\sqrt{\bar{k}_{j} \bar{h} \tanh \bar{k}_{j} \bar{h}}-\bar{\omega}_{j l}^{-} \\
\bar{\omega}_{j}=\frac{\omega_{j}}{\sqrt{g / h}}=\sqrt{\bar{k}_{j} \tanh \bar{k}_{j} \bar{h}}
\end{gathered}
$$

the nondimensional time, $\bar{t}=t \sqrt{g / h}$, is not necessary to describe the force amplitudes, but will be included in some of the expressions presented hereafter.

Though this section is about a fixed cylinder, the low-frequency second-order wave loads are usually only important for floating structures, as they may excite the natural frequencies of some degrees of freedom. However, it is reasonable to expect that the qualitative conclusions that can be drawn from this simpler case should also apply in some degree to a floating cylinder.

Therefore, the ranges of $\bar{k}_{j}, \bar{h}$ and $\bar{\omega}_{j l}^{-}$of interest are given by the values that are typically observed for floating structures. Thinking about floating offshore wind turbines, the water depth is usually between $50 \mathrm{~m}$ and $300 \mathrm{~m}$; the typical natural period of surge for spread moored units ranges between $60 \mathrm{~s}$ (in shallower waters) and $120 \mathrm{~s}$ (in deeper waters); and the waves of interest have periods ranging from a few seconds to about $20 \mathrm{~s}$. Giving some margin to the values, $\bar{h}$ is expected to be between 3 for large diameter cylinders in shallow waters and 60 to slender cylinders in intermediate water depth; $\bar{\omega}_{j l}^{-}$should be in the range $0.1-0.6$; and $\bar{k}_{j}$ between 0.05 and 0.95 (alternatively, $\lambda_{j} / D=\pi / \bar{k}_{j}$ in the range $3-50)$. Different values are expected as the concepts evolve to be installed in deeper waters or when slow pitch, whose natural period is usually significantly lower than the ones for surge, is of interest.

The nondimensional expressions of the forces are provided in the following sections following three different approaches: the semi-analytic solution considering diffraction theory presented by Kim and Yue (1990), which is exact to second-order in the context of potential flow; the slender-body approximation discussed in Chapter 3; and Newman's approximation, which consists in evaluating the difference-frequency second-order forces from the mean wave forces. The results obtained with these three approaches are then compared, evidencing the conditions in which each of the approximations better reproduces the exact solution.

As the comparisons are made considering the complex force amplitudes, it is important to understand the physical meaning of their real and imaginary parts. For such, consider the difference-frequency second-order velocity potential of waves propagating along the positive $\mathrm{x}$ direction:

$$
\varphi^{-}=\Re\left\{\sum_{j=1}^{N} \sum_{l=1}^{N} A_{j} A_{l}^{*} \varphi_{j l}^{-} e^{-i \omega_{j l}^{-} t}\right\}
$$


with

$$
\begin{gathered}
\varphi_{j l}^{-}=\frac{1}{2}\left(\gamma_{j l}^{-}+\gamma_{l j}^{-*}\right) \frac{\cosh \left(k_{j l}^{-}(Z+h)\right)}{\cosh \left(k_{j l}^{-} h\right)} e^{i k_{j l}^{-} x} \\
\gamma_{j l}^{-}=-\frac{i g}{2 \omega_{j}} \frac{k_{j}^{2}\left[1-\tanh ^{2}\left(k_{j} h\right)\right]-2 k_{j} k_{l}\left[1+\tanh \left(k_{j} h\right) \tanh \left(k_{l} h\right)\right]}{\omega_{j l}^{-2} / g-k_{j l}^{-} \tanh \left(k_{j l}^{-} h\right)}
\end{gathered}
$$

It can be shown that $\left|\gamma_{j l}^{-}\right|<\left|\gamma_{l j}^{-}\right|$when $\omega_{j}>\omega_{l}$, meaning that the complex amplitude of the second-order potential for unitary wave amplitude, $\varphi_{j l}^{-}$, is a positive imaginary number at $x=0$. As expected, the contrary occurs when $\omega_{j}<\omega_{l}$, because since the pairs $j l$ and $l j$ actually correspond to the same wave, their contributions to $\varphi^{-}$must be the same, meaning that $\varphi_{j l}^{-}=\varphi_{l j}^{-*}$. Other quantities of interest have the following phases:

- The second-order incoming wave elevation is given by:

$$
\eta_{I}^{-}=\Re\left\{\sum_{j=1}^{N} \sum_{l=1}^{N} A_{j} A_{l}^{*} \zeta_{I, j l}^{-} e^{-i \omega_{j l}^{-} t}\right\}
$$

with

$$
\zeta_{I, j l}^{-}=-\frac{1}{4 g} \nabla \varphi_{j}^{(1)} \cdot \nabla \varphi_{l}^{(1) *}-\frac{\omega_{j} \omega_{l}\left(\omega_{j}^{2}+\omega_{l}^{2}\right)}{4 g^{3}} \varphi_{j}^{(1)} \varphi_{l}^{(1) *}+\frac{i \omega_{j l}^{-} \varphi_{j l}^{-}}{g}
$$

and $\varphi_{j}^{(1)}$ the complex amplitude of the first-order incoming wave potential, given in Equation 3.33. The resulting $\zeta_{I, j l}^{-}$is a negative real number when evaluated at $x=0$;

- The amplitude of the second-order horizontal fluid acceleration due to the incoming wave is obtained from $\varphi_{j l}^{-}$by deriving once with respect to $x$ (multiplication by $k_{j l}^{-} i$ ) and once with respect to $t$ (multiplication by $-\omega_{j l}^{-} i$ ). Thus, it is a positive imaginary number at $x=0$, which is equivalent to a $-90^{\circ}$ phase with respect to $\zeta_{I, j l}^{-}$;

- The difference-frequency second-order pressure due to the incoming wave, which is calculated as follows:

$$
P_{I}^{-}=\Re\left\{\sum_{j=1}^{N} \sum_{l=1}^{N} A_{j} A_{l}^{*} p_{I, j l}^{-} e^{-i \omega_{j l}^{-} t}\right\}
$$

where

$$
p_{I, j l}^{-}=-\frac{1}{4} \rho \nabla \varphi_{j}^{(1)} \cdot \nabla \varphi_{l}^{(1) *}+i \rho \omega_{j l}^{-} \varphi_{j l}^{-}
$$

is a negative real number at $x=0$, hence in phase with the second-order wave elevation; 


\subsubsection{Solution with three different approaches}

The difference-frequency second-order wave load at a given degree of freedom $\alpha$ has the following form:

$$
F_{\alpha}^{(2)}(t)=\Re\left\{\sum_{j=1}^{N} \sum_{l=1}^{N} A_{j} A_{l}^{*} f_{\alpha, j l}^{-} \mathrm{e}^{-i \omega_{j l}^{-} t}\right\}
$$

where $f_{\alpha, j l}^{-}$is the difference-frequency force/moment quadratic transfer function (QTF) at the degree of freedom $\alpha$. In the particular case treated here, there is only a horizontal force acting along the direction of the waves, $\alpha=1$, and a corresponding turning moment, $\alpha=5$.

Since the cylinder is fixed, the force (or moment) QTF is given by:

$$
f_{j l}^{-}=f_{I, V^{2}, j l}^{-}+f_{P, V^{2}, j l}^{-}+f_{I, \eta, j l}^{-}+f_{P, \eta, j l}^{-}+f_{I, \phi^{(2)}, j l}^{-}+f_{P, \phi^{(2)}, j l}^{-}
$$

with $I$ indicating the part of the force related to the undisturbed incident flow and $P$ the one associated with the perturbation caused by the body. The index $V^{2}$ identifies the force due to the first-order velocity squared, while $\eta$ is the one due to the first-order wave elevation and $\phi^{(2)}$ the one originating from the second-order potential, as detailed ahead. Strictly speaking, there is also an additional free surface forcing term, but since it is known to be negligible in this case (KIM; YUE, 1990; KIM; CHEN, 1994; MOLIN; CHEN, 2002) and its computation is very complex, it is discarded. These components can be computed with the three approaches below.

\subsubsection{Diffraction theory}

The incident part of the horizontal forces are given by:

$$
\begin{aligned}
f_{I, V^{2}, j l}^{-} & =-\frac{\rho}{4} \int_{-h}^{0} \int_{0}^{2 \pi}\left(\nabla \varphi_{j}^{(1)} \cdot \nabla \varphi_{l}^{(1) *}\right)_{r=R} R \cos \theta \mathrm{d} \theta \mathrm{d} Z \\
f_{I, \eta, j l}^{-} & =\frac{\rho \omega_{j} \omega_{l}}{4 g} \int_{-\pi}^{\pi}\left(\varphi_{j}^{(1)} \varphi_{l}^{(1) *}\right)_{\substack{r=R \\
z=0}} R \cos \theta \mathrm{d} \theta \\
f_{I, \phi^{(2)}, j l}^{-} & =i \rho \omega \int_{-h}^{0} \int_{0}^{2 \pi} \varphi_{j l}^{-} R \cos \theta \mathrm{d} \theta \mathrm{d} Z
\end{aligned}
$$

with $\varphi_{j}^{(1)}$ the complex amplitude of the first-order incident wave potential for unitary wave amplitude, i.e. $\varphi_{j}^{(1)}=\Re\left\{A_{j} \varphi_{j}^{(1)} e^{-i \omega_{j} t}\right\}$. The corresponding components due to the perturbation caused by the body are:

$$
f_{P, V^{2}, j l}^{-}=f_{V^{2}, j l}^{-}-f_{I, V^{2}, j l}^{-}
$$




$$
\begin{aligned}
f_{P, \eta, j l}^{-} & =f_{\eta, j l}^{-}-f_{I, \eta, j l}^{-} \\
f_{P, \phi^{(2)}, j l}^{-} & =i \rho \omega \int_{-h}^{0} \int_{0}^{2 \pi} \tilde{\varphi}_{j l}^{-} R \cos \theta \mathrm{d} \theta \mathrm{d} Z
\end{aligned}
$$

where $f_{V^{2}, j l}^{-}$and $f_{\eta, j l}^{-}$are the forces due to the total first-order potential, obtained considering $\Phi_{j}^{(1)}=\varphi_{j}^{(1)}+\tilde{\varphi}_{j}^{(1)}$ instead of $\varphi_{j}^{(1)}$ in Equations 5.13 and 5.14.

These integrals have semi-analytical solutions in terms of infinite series of Bessel functions, as presented in Appendix D. The resulting force is the same as the ones obtained by Kim and Yue (1990), with the difference that in their work the components $f_{V^{2}, j l}^{-}$and $f_{\eta, j l}^{-}$are not given separately, and it is not clear which part of their expression is due to each of these components. Besides this distinction, in Appendix D they are further split into the components due to the incoming flow, $I$, and to the body perturbation, $P$. Furthermore, the expression for the pitch moment with respect to the intersection with the mean waterline, point $E$, are also given.

As the loads are given in terms of an infinite series, it would be necessary to perform a convergence analysis in order to choose the number of modes $n$. Fortunately, this work was already conducted by Kim and Yue (1990), and a number of modes $n=10$ is more than enough for all the cases analyzed in this thesis. Actually, fewer modes would be enough, but since the computation time required to calculate these expressions is negligible, reducing $n$ is unnecessary.

The resulting nondimensional total difference-frequency force, $\bar{f}_{j l}^{-}=f_{j l}^{-} /(\rho g R)$, and moment, $\bar{m}_{j l}^{-}=m_{j l}^{-} /(\rho g R h)$, amplitude QTFs are given in Figure 10 for $\bar{\omega}_{j l}^{-}=0.300$ and different values of $\bar{h}$, while Figure 11 presents the results for $\bar{h}=15$ and some values of $\bar{\omega}_{j l}^{-}$, with and without accounting for the contribution of the second-order potential. For visualization purposes, the results are given as a function of $\lambda_{j} / D$ instead of $\bar{k}_{j}$. The graphs illustrate that the loads due to the second-order potential are increasingly important as the nondimensional water depth reduces or as the nondimensional difference-frequency increases, in such a way that the QTFs in long waves can be comparable or even larger than the ones observed in short waves, which are more affected by diffraction effect. Due the slower depth attenuation of the second-order potential, this is more pronounced for the moment, as it is given with respect to $Z=0$.

The separate contributions to the QTF of the different force components are given in Section 5.1.2 in the comparisons with the results from the other two approaches. 
Figure 10 - Nondimensional force and moment QTFs calculated with diffraction theory for $\bar{\omega}_{j l}^{-}=0.300$ and different values of $\bar{h}$.
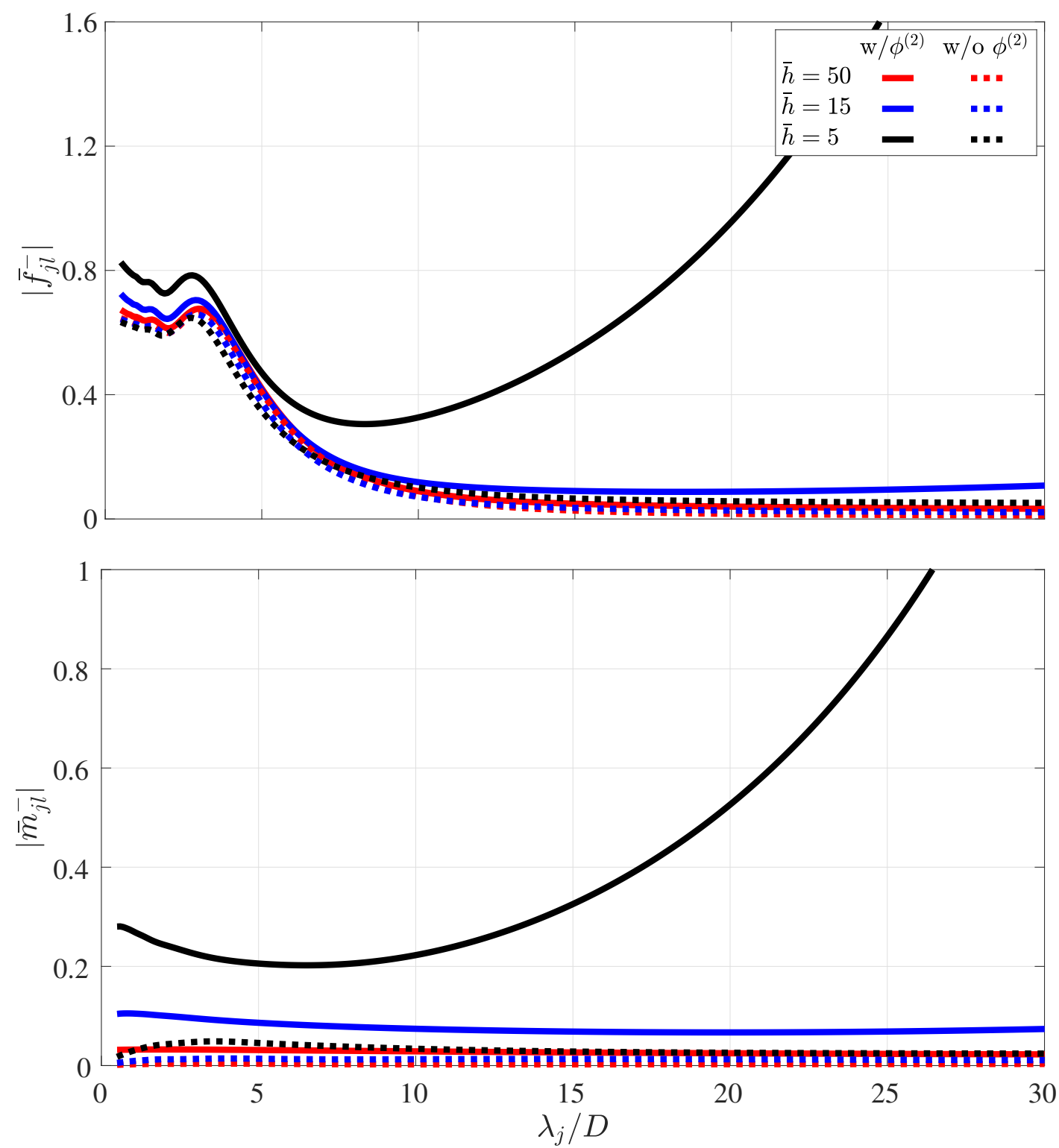
Figure 11 - Nondimensional force and moment QTFs calculated with diffraction theory for $\bar{h}=15$ and different values of $\bar{\omega}_{j l}^{-}$.
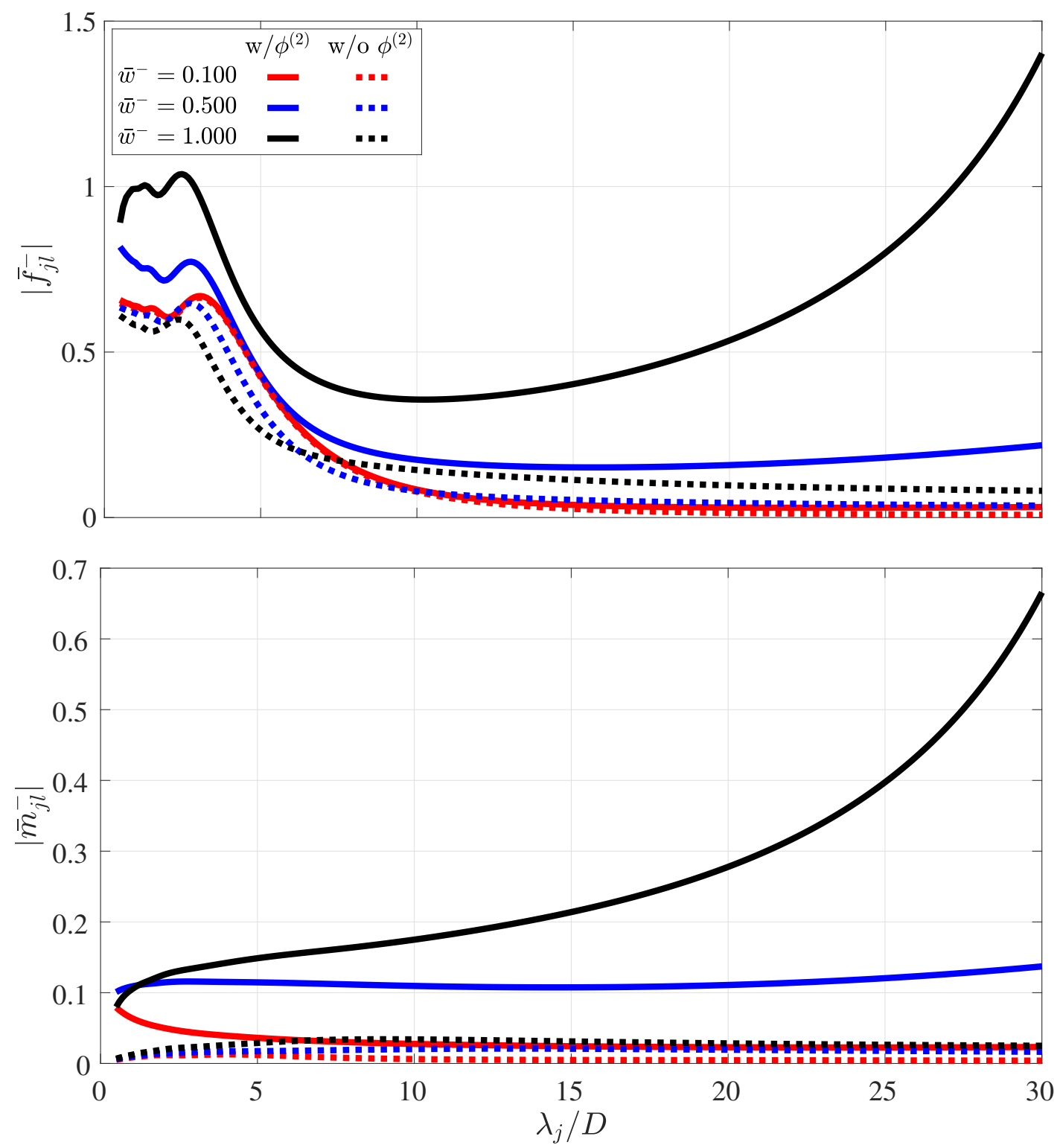

\subsubsection{Slender-body approximation}

Since the cylinder is bottom mounted, there are no hydrodynamic forces acting at its extremities and no effects due to body motions, hence only four of the second-order components presented in Section 3.6 need to be considered. The necessary kinematic quantities are obtained from the first- and second-order incident wave potentials given by Equations 3.33 and 3.35 considering that the waves are propagating along the positive $\mathrm{X}$ direction, i.e. $\beta_{j}=0$. The procedure is way simpler than the one presented in the previous section, as only an integration along the $Z$ axis is needed, hence only the final expressions are given below:

Force due to the first-order incoming flow: recalling Equation 3.102 and retaining 
only the force component along the $\mathrm{X}$ direction:

$$
\begin{aligned}
& F_{\phi^{(1)}}^{(1)}=\Re\left\{\sum_{j=1}^{N} A_{j} f_{\phi^{(1)}, j}^{(1)} e^{-i \omega_{j} t}\right\}=\rho \pi R^{2} \int_{-h}^{0}\left(1+C_{a}\right) \frac{\partial u^{(1)}}{\partial t} \mathrm{~d} Z \\
& M_{\phi^{(1)}}^{(1)}=\Re\left\{\sum_{j=1}^{N} A_{j} m_{\phi^{(1)}, j}^{(1)} e^{-i \omega_{j} t}\right\}=\rho \pi R^{2} \int_{-h}^{0}\left(1+C_{a}\right) \frac{\partial u^{(1)}}{\partial t} Z \mathrm{~d} Z
\end{aligned}
$$

leading to

$$
\begin{gathered}
\bar{f}_{\phi^{(1)}, j}^{(1)}=\frac{f_{\phi^{(1)}, j}^{(1)}}{\rho g R^{2}}=-i \pi\left(1+C_{a}\right) \tanh \left(\bar{k}_{j} \bar{h}\right) \\
\bar{m}_{\phi^{(1)}, j}^{(1)}=\frac{m_{\phi^{(1)}, j}^{(1)}}{\rho g R^{2} h}=-i \pi\left(1+C_{a}\right) \frac{\operatorname{sech}\left(\bar{k}_{j} \bar{h}\right)-1}{\bar{k}_{j} \bar{h}}
\end{gathered}
$$

which acts at the frequency of the incoming waves, as expected from a first-order quantity, and does not contribute to the low-frequency second-order wave loads.

Force due to the second-order incoming flow: same as above, but for Equation 3.103:

$$
\begin{aligned}
& F_{\phi^{-}}^{(2)}=\Re\left\{\sum_{j=1} \sum_{l=1}^{N} A_{j} A_{l}^{*} f_{\phi^{-}, j l}^{-} e^{-i \omega_{j l}^{-} t}\right\}=\rho \pi R^{2} \int_{-h}^{0}\left(1+C_{a}\right) \frac{\partial u^{(2)}}{\partial t} \mathrm{~d} Z \\
& M_{\phi^{-}}^{(2)}=\Re\left\{\sum_{j=1} \sum_{l=1}^{N} A_{j} A_{l}^{*} m_{\phi, j l}^{-} e^{-i \omega_{j l}^{-} t}\right\}=\rho \pi R^{2} \int_{-h}^{0}\left(1+C_{a}\right) \frac{\partial u^{(2)}}{\partial t} Z \mathrm{~d} Z
\end{aligned}
$$

with

$$
\begin{aligned}
\bar{f}_{\phi^{-}, j l}^{-} & =\frac{f_{\phi^{-}, j l}^{-}}{\rho g R}=-i \frac{\pi}{2}\left(1+C_{a}\right) \frac{\bar{\omega}_{j l}^{-} \overline{\mathcal{A}}_{j l} \tanh \left|\bar{k}_{j l}^{-} \bar{h}\right|}{\left|\bar{k}_{j l}^{-} \bar{h}\right| \tanh \left|\bar{k}_{j l}^{-\bar{h}}\right|-\omega_{j l}^{-2}} \frac{1}{\bar{h}} \frac{\bar{k}_{j l}^{-}}{\left|\bar{k}_{j l}^{-}\right|} \\
\bar{m}_{\phi^{-}, j l}^{-} & =\frac{m_{\phi^{-}, j l}^{-}}{\rho g R h}=-i \frac{\pi}{2}\left(1+C_{a}\right) \frac{\bar{\omega}_{j l}^{-} \overline{\mathcal{A}}_{j l}\left(\operatorname{sech}\left|\bar{k}_{j l}^{-} \bar{h}\right|-1\right)}{\left|\bar{k}_{j l}^{-} \bar{h}\right| \tanh \left|\bar{k}_{j l}^{-} \bar{h}\right|-\omega_{j l}^{-2}} \frac{1}{\left|\bar{k}_{j l}^{-} \bar{h}\right|} \frac{1}{\bar{h}}
\end{aligned}
$$

and

$$
\begin{aligned}
\overline{\mathcal{A}}_{j l}= & \left(\frac{1}{\bar{\omega}_{j}}-\frac{1}{\bar{\omega}_{l}}\right)\left(\bar{k}_{j} \bar{h}\right)\left(\bar{k}_{l} \bar{h}\right)\left[1+\tanh \left(\bar{k}_{j} \bar{h}\right) \tanh \left(\bar{k}_{l} \bar{h}\right)\right] \\
& -\frac{1}{2}\left(\frac{\left(\bar{k}_{j} \bar{h}\right)^{2}}{\bar{\omega}_{j} \cosh ^{2}\left(\bar{k}_{j} \bar{h}\right)}-\frac{\left(\bar{k}_{l} \bar{h}\right)^{2}}{\bar{\omega}_{l} \cosh ^{2}\left(\bar{k}_{l} \bar{h}\right)}\right)
\end{aligned}
$$

which results in zero loads when $j=l$, thus they do not contribute to the mean force and moment. As only the second-order potential at the difference-frequency was considered, there are no loads at the sum-frequencies. 
Force due to the convective acceleration: from Equation 3.104:

$$
\begin{aligned}
F_{\mathrm{conv}}^{(2)} & =\Re\left\{\sum_{j=1}^{N} \sum_{l=1}^{N} A_{j} A_{l}^{*} f_{\text {conv }, j l}^{-} e^{-i \omega_{j l}^{-} t}+A_{j} A_{l} f_{\text {conv }, j l}^{+} e^{-i \omega_{j l}^{+} t}\right\} \\
& =\rho \pi R^{2} \int_{-h}^{0}\left(1+C_{a}\right)\left(u^{(1)} \frac{\partial u^{(1)}}{\partial x}+w^{(1)} \frac{\partial u^{(1)}}{\partial z}\right) \mathrm{d} Z \\
M_{\mathrm{conv}}^{(2)} & =\Re\left\{\sum_{j=1}^{N} \sum_{l=1}^{N} A_{j} A_{l}^{*} m_{\mathrm{conv}, j l}^{-} e^{-i \omega_{j l}^{-} t}+A_{j} A_{l} m_{\mathrm{conv}, j l}^{+} e^{-i \omega_{j l}^{+} t}\right\} \\
& =\rho \pi R^{2} \int_{-h}^{0}\left(1+C_{a}\right)\left(u^{(1)} \frac{\partial u^{(1)}}{\partial x}+w^{(1)} \frac{\partial u^{(1)}}{\partial z}\right) Z \mathrm{~d} Z
\end{aligned}
$$

Retaining only the difference-frequency part of the force, one obtains:

$$
\begin{gathered}
\bar{f}_{\text {conv }, j l}^{-}=\frac{f_{\text {conv }, j l}^{-}}{\rho g R}=i \frac{\pi}{4}\left(1+C_{a}\right) \frac{\left(\bar{k}_{j} \bar{h}\right)\left(\bar{k}_{l} \bar{h}\right)}{\bar{\omega}_{j} \bar{\omega}_{l}} \frac{\tanh \left(\bar{k}_{j} \bar{h}\right)+\tanh \left(\bar{k}_{l} \bar{h}\right)}{\bar{k}_{j l}^{+} \bar{h}} \bar{k}_{j l}^{-} \\
\bar{m}_{\text {conv }, j l}^{-}=\frac{m_{\text {conv }, j l}^{-}}{\rho g R h}=i \frac{\pi}{4}\left(1+C_{a}\right) \frac{\left(\bar{k}_{j} \bar{h}\right)\left(\bar{k}_{l} \bar{h}\right)}{\bar{\omega}_{j} \bar{\omega}_{l}} \frac{1}{\cosh \left(\bar{k}_{j} \bar{h}\right) \cosh \left(\bar{k}_{l} \bar{h}\right)} \frac{1-\cosh \left(\bar{k}_{j l}^{+} \bar{h}\right)}{\left(\bar{k}_{j l}^{+} \bar{h}\right)^{2}} \bar{k}_{j l}^{-}
\end{gathered}
$$

Force due to the axial-divergence acceleration: from Equation 3.106:

$$
\begin{aligned}
F_{\mathrm{axdv}}^{(2)} & =\Re\left\{\sum_{j=1}^{N} \sum_{l=1}^{N} A_{j} A_{l}^{*} f_{\mathrm{axdv}, j l}^{-} e^{-i \omega_{j l}^{-} t}+A_{j} A_{l} f_{\mathrm{axdv}, j l}^{+} e^{-i \omega_{j l}^{+} t}\right\} \\
& =\rho \pi R^{2} \int_{-h}^{0} C_{a} \frac{\partial w^{(1)}}{\partial z} u^{(1)} \mathrm{d} Z \\
M_{\mathrm{axdv}}^{(2)} & =\Re\left\{\sum_{j=1}^{N} \sum_{l=1}^{N} A_{j} A_{l}^{*} m_{\mathrm{axdv}, j l}^{-} e^{-i \omega_{j l}^{-} t}+A_{j} A_{l} m_{\mathrm{axdv}, j l}^{+} e^{-i \omega_{j l}^{+} t}\right\} \\
& =\rho \pi R^{2} \int_{-h}^{0} C_{a} \frac{\partial w^{(1)}}{\partial z} u^{(1)} Z \mathrm{~d} Z
\end{aligned}
$$

with

$$
\bar{f}_{\mathrm{axdv}, j l}^{-}=\frac{f_{\mathrm{axdv}, j l}^{-}}{\rho g R}=-i \frac{\pi}{4} C_{a} \frac{\left(\bar{k}_{j} \bar{h}\right)\left(\bar{k}_{l} \bar{h}\right)}{\bar{\omega}_{j} \bar{\omega}_{l}} \frac{\bar{k}_{j} \bar{h} \tanh \left(\bar{k}_{j} \bar{h}\right)-\bar{k}_{l} \bar{h} \tanh \left(\bar{k}_{l} \bar{h}\right)}{\left(\bar{k}_{j l}^{-} \bar{h}\right)\left(\bar{k}_{j l}^{+} \bar{h}\right)} \bar{k}_{j l}^{-}
$$




$$
\begin{aligned}
\bar{m}_{\mathrm{axdv}, j l}^{-}=\frac{m_{\mathrm{axdv}, j l}^{-}}{\rho g R h}= & -i \frac{\pi}{8} C_{a} \frac{\left(\bar{k}_{j} \bar{h}\right)\left(\bar{k}_{l} \bar{h}\right)}{\bar{\omega}_{j} \bar{\omega}_{l}} \frac{1}{\cosh \left(\bar{k}_{j} \bar{h}\right) \cosh \left(\bar{k}_{l} \bar{h}\right)} \\
& \cdot\left[\frac{1-\cosh \left(\bar{k}_{j l}^{-} \bar{h}\right)}{\left(\bar{k}_{j l}^{-} \bar{h}\right)^{2}}+\frac{1-\cosh \left(\bar{k}_{j l}^{+} \bar{h}\right)}{\left(\bar{k}_{j l}^{+} \bar{h}\right)^{2}}\right] \bar{k}_{j l}^{-}
\end{aligned}
$$

Force due to the wave elevation: finally, Equation 3.109 yields:

$$
F_{\eta}^{(2)}=\Re\left\{\sum_{j=1}^{N} \sum_{l=1}^{N} A_{j} A_{l}^{*} f_{\eta, j l}^{-} e^{-i \omega_{j l}^{-} t}+A_{j} A_{l} f_{\eta, j l}^{+} e^{-i \omega_{j l}^{+} t}\right\}=\left.\rho \pi R^{2}\left(1+C_{a}\right) \eta^{(1)} \frac{\partial u^{(1)}}{\partial t}\right|_{z=0}
$$

where

$$
\bar{f}_{\eta, j l}^{-}=\frac{f_{\eta, j l}^{-}}{\rho g R}=-i \frac{\pi}{4}\left(1+C_{a}\right) \bar{k}_{j l}^{-}
$$

with the corresponding moment with respect to the mean water line equal to zero.

It is remarkable that the force amplitudes calculated with the slender-body approximation are purely imaginary numbers, and an immediate consequence is that they do not contribute to the mean force, a clear contradiction with the non-zero result that is obtained with their counterparts from diffraction theory.

As discussed in Chapter 3, the part of the force that is multiplied by $C_{a}$ originates from the flow perturbation due to the presence of the body. Besides, both the force due to the convective acceleration and the one due to the axial-divergence acceleration originate from the $(\nabla \phi)^{2}$ term of Bernoulli's equation. Hence, the force amplitudes above can be rearranged to match Equation 5.12 as follows:

$$
\begin{aligned}
\bar{f}_{I, \phi^{(2)}, j l}^{-} & =\frac{\bar{f}_{\phi^{-}, j l}^{-}}{\left(1+C_{a}\right)} \\
\bar{f}_{P, \phi^{(2)}, j l}^{-} & =\frac{C_{a} \bar{f}_{\phi^{-}, j l}^{-}}{\left(1+C_{a}\right)} \\
\bar{f}_{I, V^{2}, j l}^{-} & =\frac{\bar{f}_{\text {conv }, j l}^{-}}{\left(1+C_{a}\right)} \\
\bar{f}_{P, V^{2}, j l}^{-} & =C_{a}\left(\frac{\bar{f}_{\text {conv }, j l}^{-}}{\left(1+C_{a}\right)}+\bar{f}_{\mathrm{axdv}, j l}^{-}\right) \\
\bar{f}_{I, \eta, j l}^{-} & =\frac{\bar{f}_{\eta, j l}^{-}}{\left(1+C_{a}\right)} \\
\bar{f}_{P, \eta, j l}^{-} & =\frac{C_{a} \bar{f}_{\eta, j l}^{-}}{\left(1+C_{a}\right)}
\end{aligned}
$$

where the added mass coefficient is taken as $C_{a}=1$, which is the value calculated with potential theory neglecting the effect of the free surface. The same applies for the moments. 
One interesting aspect of the slender-body approximation is that it allows the direct examination of the contribution of each force component along the length of the cylinder, as illustrated in Figure 12, which evidences the slower decay of the component due to the second-order potential with respect to the others. It also shows the axial-divergence acceleration acting in the opposite direction of the convective acceleration, reducing the resulting $f_{P, V^{2}, j l}^{-}$.

Figure 12 - Nondimensional force per unit length along the cylinder for $\bar{h}=10, \bar{\omega}_{j l}^{-}=0.300$ and $\bar{k}_{j}=\pi / 10$ (i.e. $\lambda_{j} / D=10$ ).

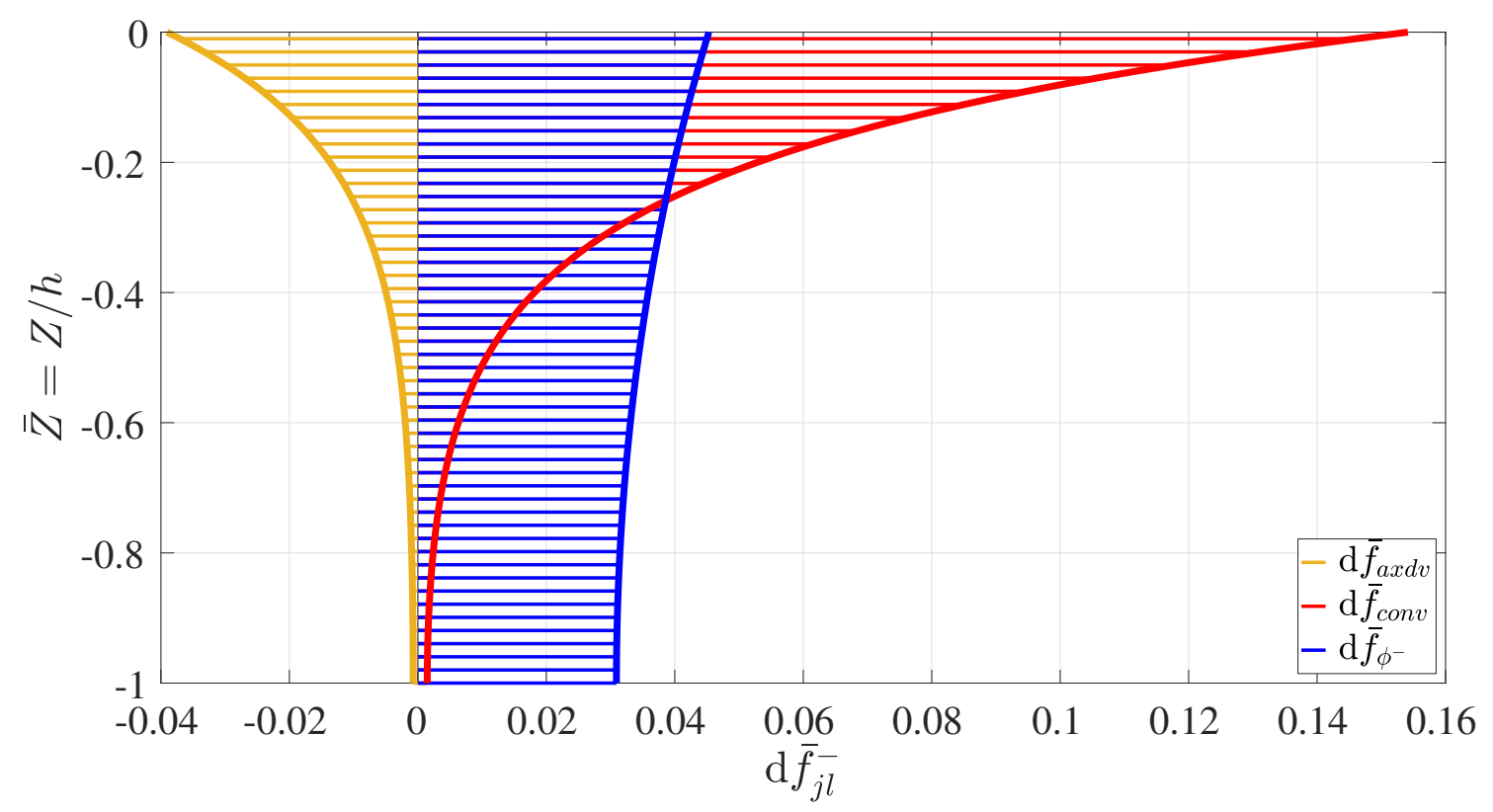

\subsubsection{Solution using Newman's approximation}

Among the existing approximations for the evaluation of the difference-frequency secondorder wave forces, Newman's approximation (NEWMAN, 1974) is probably the most used. By assuming that the difference-frequencies $\omega_{j l}^{-}$of interest are small compared to the average frequency $\omega_{j l}^{+} / 2$, the approximation evaluates the low-frequency forces $f_{j l}^{-}$from the mean forces $f_{j j}^{-}$and $f_{l l}^{-}$as follows ( $\Im$ denotes the imaginary part):

$$
\begin{aligned}
& \Re\left\{f_{j l}^{-}\right\}=\Re\left\{f_{l j}^{-}\right\}=\Re\left\{\frac{f_{j j}^{-}+f_{l l}^{-}}{2}\right\} \\
& \Im\left\{f_{j l}^{-}\right\}=\Im\left\{f_{l j}^{-}\right\}=0
\end{aligned}
$$

which greatly reduces the complexity of the problem, as the mean forces do not depend on the second-order velocity potential. This can be further simplified by approximating the double summation from Equation 5.11 by the square of a single series, but this is not necessary for the purposes of this chapter. The expressions for $f_{j j}^{-}$are given in Appendix D. 
Since the low-frequency forces are obtained from the mean forces, whose imaginary part is zero, only the real part is captured by the approximation, in opposition to the results calculated with the slender-body approximation (MOLIN; CHEN, 2002).

\subsubsection{Comparisons considering bichromatic waves}

The forces acting on the cylinder calculated with each of the three different methods discussed in the previous sections are compared in Figure 13 and Table 1, for $\bar{\omega}_{j l}^{-}=0.300$, $\bar{h}=20$ and $\lambda_{j} / D$ from 0 to 20 . This combination of parameters was chosen for visualization purposes only, but the observations below are valid for all the combinations within the ranges that were tested, which are $0.05 \leq \bar{\omega}_{j l}^{-} \leq 1,1 \leq \bar{h} \leq 60$ and $0 \leq \lambda_{j} / D \leq 60$. They are probably valid for larger ranges, but these are the ones that were verified.

The first graph of Figure 13 shows that the real part of the exact solution, which is completely insensitive to the second-order potential, is practically identical to the force calculated with Newman's approximation, except for small discrepancies in waves shorter than one diameter. On the other hand, the slender-body approximation matches very well the imaginary part of the force, though it fails to capture a broad peak around $\lambda_{j} / D \approx 3$, whether the second-order potential is included or not. As illustrated by the absolute value of the force, given in the second graph of Figure 13, the slender-body approximation performs better as the wave length increases and the real part of the force becomes increasingly irrelevant, in opposition to what is observed for the correspondence between Newman's approximation and the force calculated without the second-order potential. Though the ranges of $\lambda_{j} / D$ for which each approximation performs better are dependent on $\bar{\omega}_{j l}^{-}$and $\bar{h}$, as they impact the relative importance between the real and imaginary parts of the force, it seems that $\lambda_{j} / D>10$ is a good general threshold for the validity of the slender-body approximation. The best results are obtained by summing both approximations, as in this way both the real and imaginary parts are well captured.

A better understanding can be gained by analyzing the different components that constitute the force, as illustrated in Figure 14. The forces due to the undisturbed incident waves are purely imaginary numbers, and the values calculated with the slender-body approximation are practically equal to the exact solution using diffraction theory, even in short waves. The contributions of the first-order perturbation potential, however, have a real part that increases as the waves get shorter and wave scattering becomes more relevant. Since the perturbation predicted by the slender-body approximation is obtained neglecting the boundary condition at the free surface, it is natural that it fails to model this effect. As scattering effects are negligible for the second-order potential, the real part of $\bar{f}_{\phi^{(2)}}$ is practically zero. The agreement of the imaginary part of the perturbation forces is not as good as for the incident part, as they diverge in short waves, and this explains the differences noted in Figure 13, specially for the peak around $\lambda_{j} / D=3$ that originates 
Figure 13 - Nondimensional horizontal force acting on the bottom mounted cylinder calculated with diffraction theory, the slender-body and Newman's approximations for $\bar{\omega}_{j l}^{-}=0.300$ and $\bar{h}=20$.
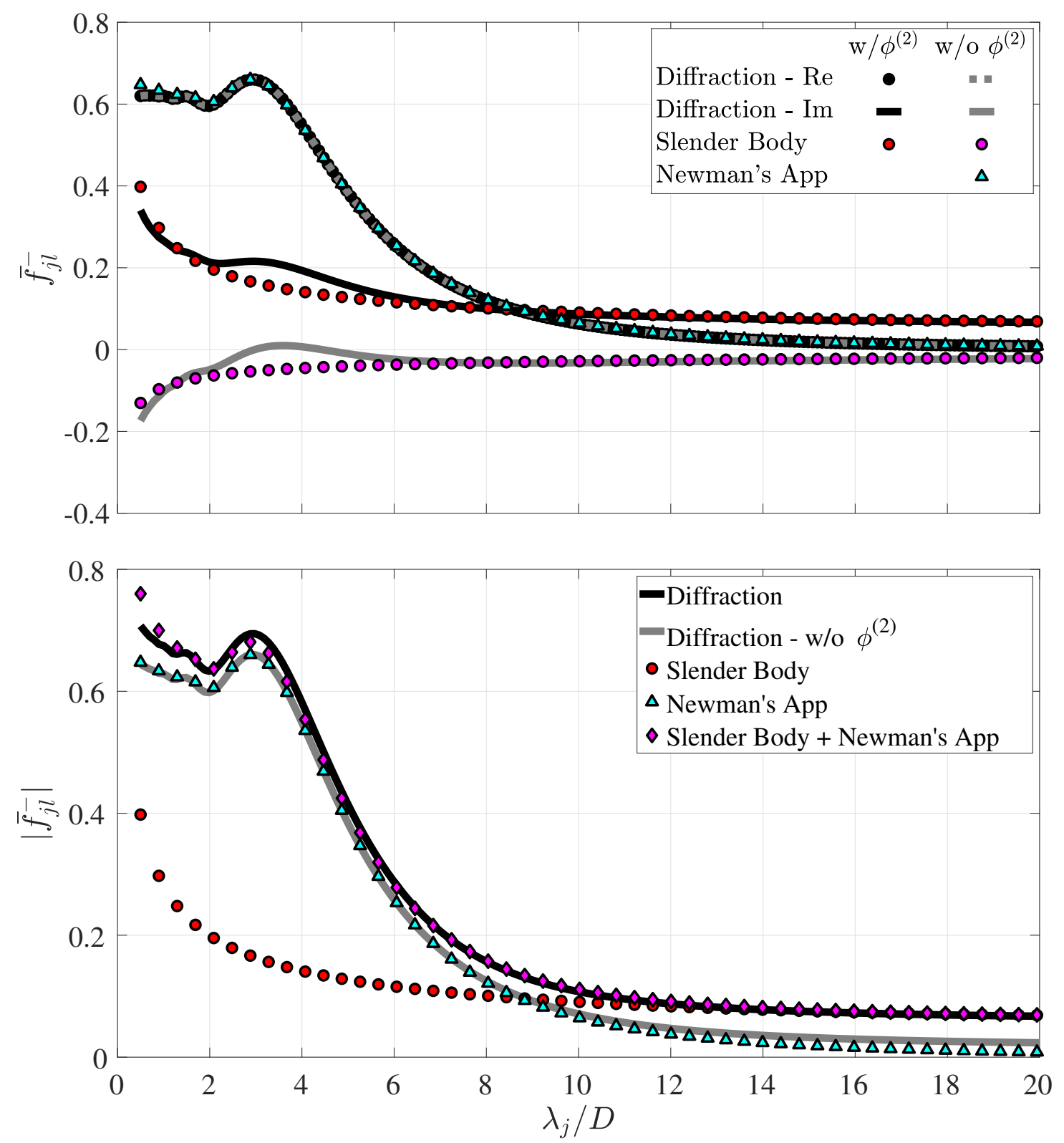

Table 1 - Selected points from the first graph of Figure 13 to ease the comparisons.

\begin{tabular}{|c|c|c|c|c|c|c|c|}
\hline$\lambda_{1} / D$ & \multicolumn{2}{|c|}{ Diffraction - Real } & \multicolumn{2}{|c|}{ Diffraction - Imag. } & $\begin{array}{r}\text { Slen } \\
\mathrm{w} / \phi^{(2)}\end{array}$ & $\begin{array}{l}\text { Body } \\
\mathrm{w} / \mathrm{o} \phi^{(2)}\end{array}$ & $\begin{array}{c}\text { Newman's } \\
\text { approx. }\end{array}$ \\
\hline 5 & 0.384 & 0.384 & 0.157 & -0.010 & 0.127 & -0.040 & 0.384 \\
\hline 10 & 0.063 & 0.063 & 0.087 & -0.032 & 0.091 & -0.028 & 0.065 \\
\hline 20 & 0.008 & 0.008 & 0.067 & -0.022 & 0.069 & -0.020 & 0.008 \\
\hline
\end{tabular}

from the force due to the wave elevation.

As the added mass coefficient is $C_{a}=1$, the perturbation forces obtained with the slender-body approximation are equal to their counterparts caused by the undisturbed 
incident potential, except for $\bar{f}_{V^{2}}$. In this case, the difference between the incident and perturbation parts is the contribution of the axial-divergence acceleration, and the fact that this difference actually appears in the results is proof that this force component, whose existence is not intuitive, is indeed correct (again, the reader is referred to the article of Kim and Chen (1994) for a brief discussion on a controversy regarding the axial-divergence term).

Figure 15 presents similar results, but for varying values of $\bar{h}$, while the other parameters are kept fixed. For visualization purposes, the force due to the second-order potential is not included in the graphs for these small values of $\bar{h}$, but they can be verified in Table 3. It is clear that the importance of the second-order potential, which is well modeled by the slender-body approximation, increases as $\bar{h}$ is reduced, meaning that better results are obtained as the water gets shallower. Once again, this behavior is complementary to Newman's approximation, which should not be used in shallow waters due to neglecting the second-order potential.

Figure 16 illustrates an equivalent analysis, but for varying values of $\bar{\omega}_{j l}^{-}$. It can be seen the second-order potential is increasingly relevant as the difference-frequency increases, which is due to the consequently larger second-order fluid acceleration, and that the results for the imaginary perturbation part of $\bar{f}_{\phi^{(2)}}$ start to slightly diverge at large values of $\bar{\omega}_{j l}^{-}$, a consequence of the length of the second-order wave becoming smaller. The real part of the second-order perturbation potential remains negligible (though different from zero) for all the analyzed values of the difference frequency, while for the other components it increases as $\bar{\omega}_{j l}^{-}$is reduced. As a consequence, the slender-body approximation performs better as $\bar{\omega}_{j l}^{-}$gets larger, which, again, is the opposite of Newman's approximation, which requires low values of the difference frequency to assure that they are evaluated close to $\bar{\omega}_{j j}^{-}=0$.

The same conclusions can be drawn for the moment with respect to the mean water line, with the difference that there is no contribution of the wave elevation and that the second-order wave potential is more relevant than for the force, improving the performance of the slender-body approximation and worsening that of Newman's approximation, as illustrated in Figure 17. 
Figure 14 - Components of the nondimensional horizontal force acting on the bottom mounted cylinder calculated with diffraction theory and the slender-body approximation for $\bar{\omega}_{j l}^{-}=0.300$ and $\bar{h}=20$.
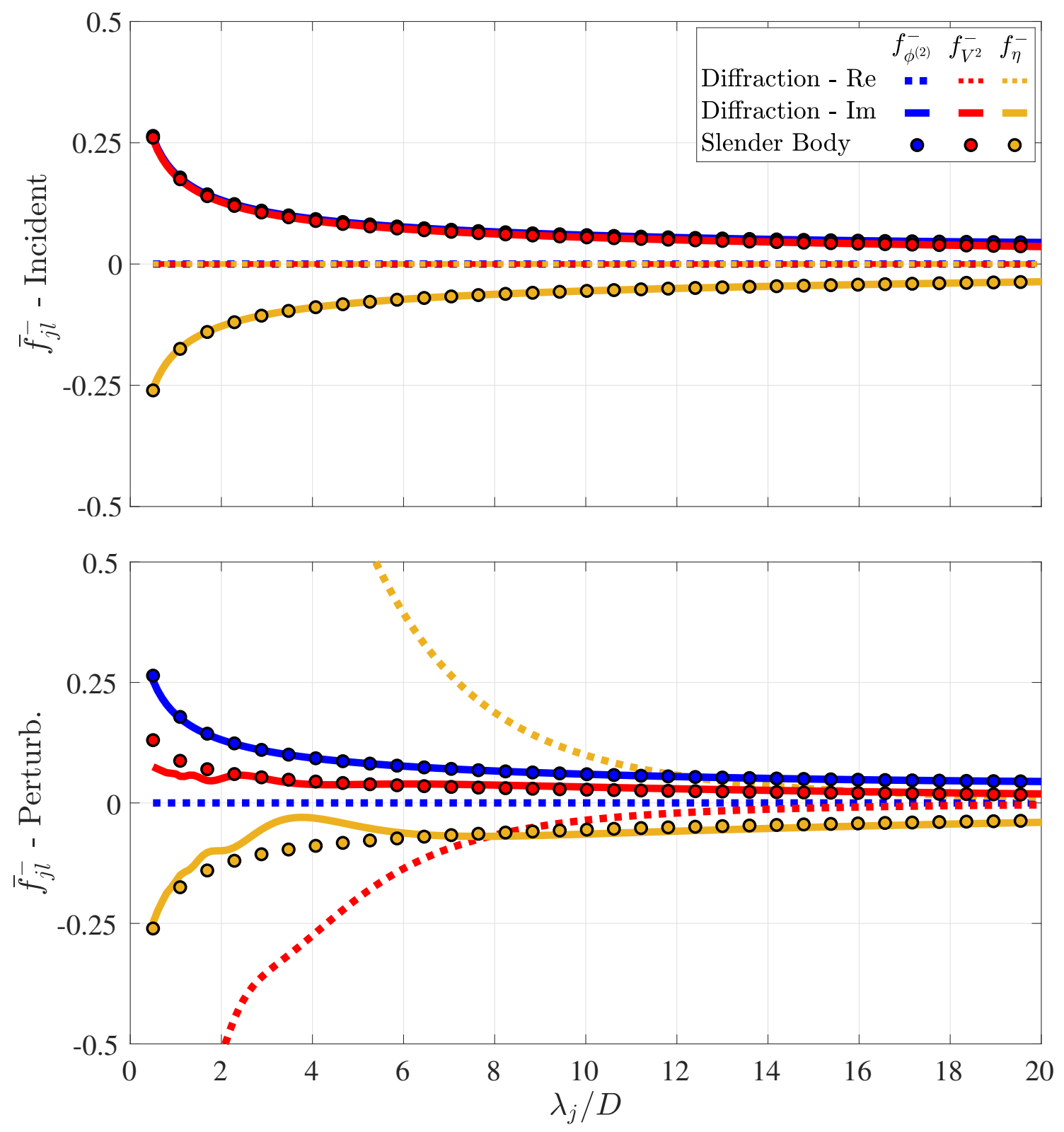

Table 2 - Imaginary part of the forces at selected points of Figure 14 .

\begin{tabular}{|c|c|c|c|c|c|c|c|}
\hline & & & ction - & & & nder b & \\
\hline & $\lambda_{j} / D$ & $\bar{f}_{\phi^{(2)}}$ & $\bar{f}_{V^{2}}$ & $\bar{f}_{\eta}$ & $\bar{f}_{\phi^{(2)}}$ & $\bar{f}_{V^{2}}$ & $\bar{f}_{\eta}$ \\
\hline$\rightleftarrows$ & 5 & 0.084 & 0.080 & -0.080 & 0.084 & 0.080 & -0.080 \\
\hline . & 10 & 0.060 & 0.055 & -0.055 & 0.060 & 0.055 & -0.056 \\
\hline$\ddot{\Xi}$ & 20 & 0.044 & 0.036 & -0.037 & 0.044 & 0.036 & -0.037 \\
\hline$\dot{0}$ & 5 & 0.083 & 0.038 & -0.048 & 0.084 & 0.040 & -0.080 \\
\hline$\underset{\nabla}{ت}$ & 10 & 0.060 & 0.033 & -0.065 & 0.060 & 0.028 & -0.056 \\
\hline$\stackrel{n}{A}$ & 20 & 0.044 & 0.019 & -0.040 & 0.044 & 0.017 & -0.037 \\
\hline
\end{tabular}


Figure 15 - Components of the nondimensional horizontal force acting on the bottom mounted cylinder calculated with diffraction theory and the slender-body approximation for $\bar{\omega}_{j l}^{-}=0.300$ and $\lambda_{j} / D=10$.
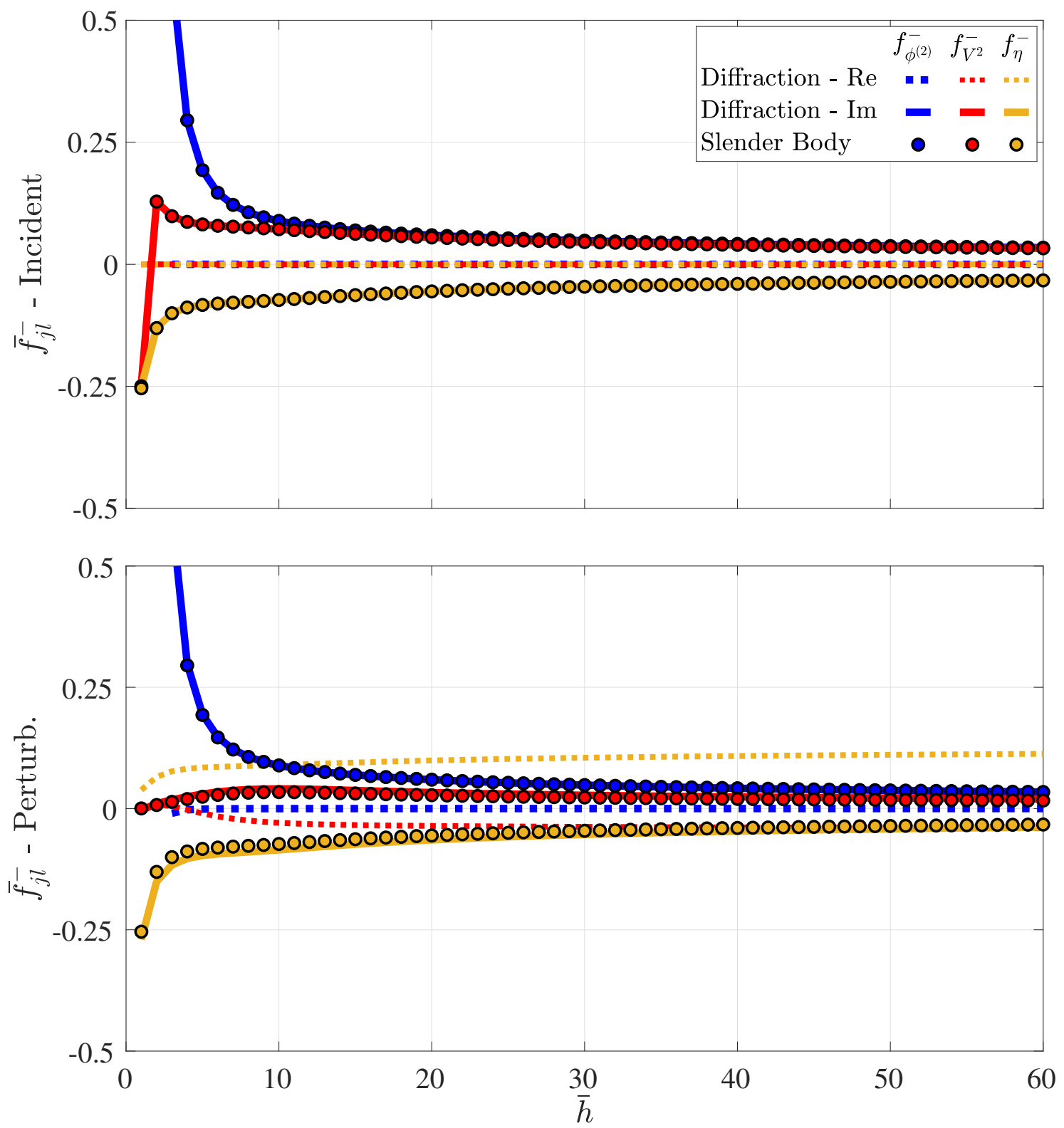

Table 3 - Imaginary part of the forces at selected points of Figure 15.

\begin{tabular}{|c|c|c|c|c|c|c|c|}
\hline \multirow{2}{*}{\multicolumn{2}{|c|}{$\bar{h}$}} & \multicolumn{3}{|c|}{ Diffraction - Imag. } & \multicolumn{3}{|c|}{ Slender body } \\
\hline & & $\bar{f}_{\phi^{(2)}}$ & $\bar{f}_{V^{2}}$ & $\bar{f}_{\eta}$ & $\bar{f}_{\phi^{(2)}}$ & $\bar{f}_{V^{2}}$ & $\bar{f}_{\eta}$ \\
\hline \multirow{3}{*}{ 苞 } & 1 & -2.157 & -0.246 & -0.251 & -2.186 & -0.250 & -0.254 \\
\hline & 2 & 2.108 & 0.128 & -0.130 & 2.115 & 0.129 & -0.131 \\
\hline & 60 & 0.034 & 0.033 & -0.033 & 0.034 & 0.033 & -0.033 \\
\hline \multirow{3}{*}{$\begin{array}{l}\dot{0} \\
\dot{\Xi} \\
\dot{\vec{n}} \\
\dot{0}\end{array}$} & 1 & -2.329 & -0.003 & -0.270 & -2.186 & 0.000 & -0.254 \\
\hline & 2 & 2.190 & 0.010 & -0.150 & 2.115 & 0.007 & -0.131 \\
\hline & 60 & 0.034 & 0.020 & -0.038 & 0.034 & 0.016 & -0.033 \\
\hline
\end{tabular}


Figure 16 - Components of the nondimensional horizontal force acting on the bottom mounted cylinder calculated with diffraction theory and the slender-body approximation for $\bar{h}=20$ and $\lambda_{j} / D=10$.
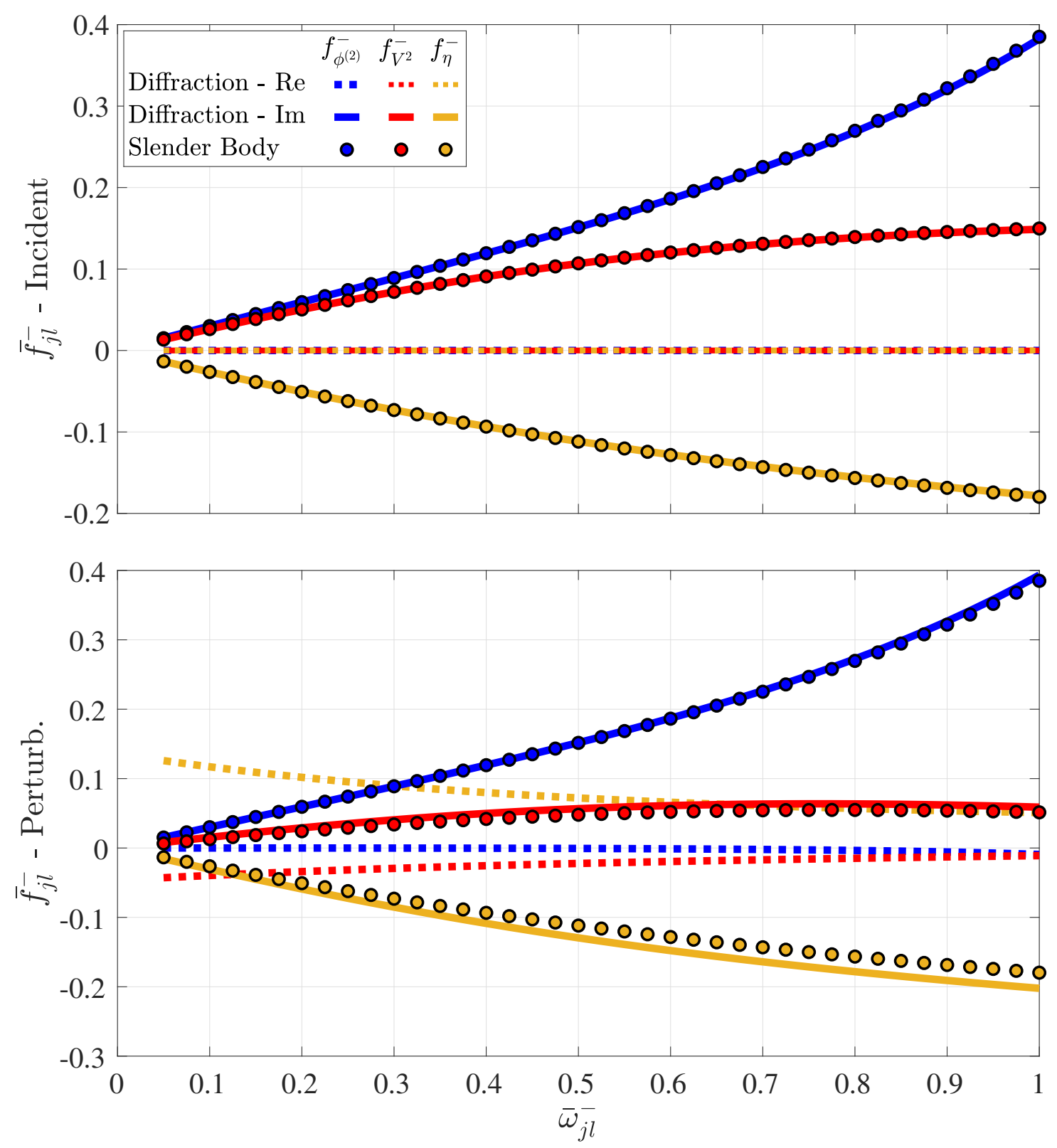

Table 4 - Imaginary part of the forces at selected points of Figure 16.

\begin{tabular}{|c|c|c|c|c|c|c|c|}
\hline \multirow{2}{*}{\multicolumn{2}{|c|}{$\bar{\omega}_{j l}^{-}$}} & \multicolumn{3}{|c|}{ Diffraction - Imag. } & \multicolumn{3}{|c|}{ Slender body } \\
\hline & & $\bar{f}_{\phi^{(2)}}$ & $\bar{f}_{V^{2}}$ & $\bar{f}_{\eta}$ & $\bar{f}_{\phi^{(2)}}$ & $\bar{f}_{V^{2}}$ & $\bar{f}_{\eta}$ \\
\hline \multirow{3}{*}{ 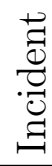 } & 0.100 & 0.030 & 0.026 & -0.026 & 0.030 & 0.026 & -0.026 \\
\hline & 0.500 & 0.151 & 0.107 & -0.112 & 0.152 & 0.107 & -0.112 \\
\hline & 1.000 & 0.383 & 0.149 & -0.179 & 0.385 & 0.150 & -0.180 \\
\hline \multirow{3}{*}{ 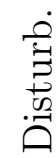 } & 0.100 & 0.030 & 0.015 & -0.031 & 0.030 & 0.013 & -0.026 \\
\hline & 0.500 & 0.152 & 0.057 & -0.130 & 0.152 & 0.048 & -0.112 \\
\hline & 1.000 & 0.393 & 0.059 & -0.202 & 0.385 & 0.051 & -0.180 \\
\hline
\end{tabular}


Figure 17 - Nondimensional moment, with respect to the mean water line, acting on the bottom mounted cylinder calculated with diffraction theory, the slender-body and Newman's approximations for $\bar{\omega}_{j l}^{-}=0.300$ and $\bar{h}=20$.
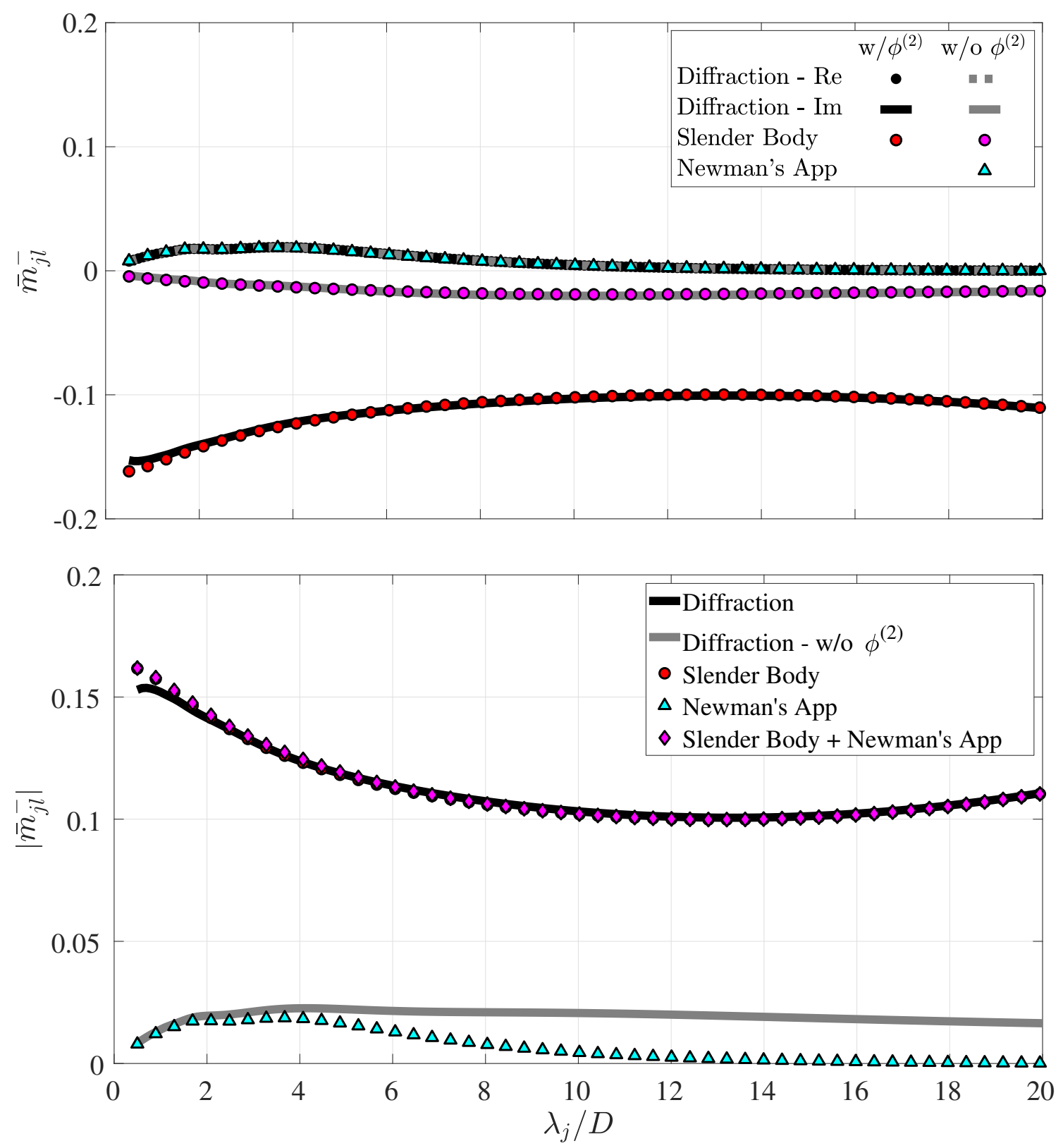

\subsection{Floating cylinder}

The problem of evaluating the second-order forces on a floating cylinder is considerably more complicated than that for a bottom mounted one, as it is necessary to take into account the effects due to the immersed extremity and to the first-order body motions. The importance of the end effects is related to the submerged length of the structure, given by the nondimensional draft, $\bar{d}=d / R$, while the motions are described by the first-order surge and heave response amplitude operators (RAOs) and the pitch motion amplitude, $\Xi_{1 j}=\Xi_{1 j} / A_{j}, \bar{\Xi}_{3 j}=\Xi_{3 j} / A_{j}$ and $\bar{\Xi}_{5 j}$, with $\xi_{\alpha}=\Re\left\{\sum_{j}^{N} \Xi_{\alpha j} e^{-i \omega_{j} t}\right\}$ (as an angular quantity, 
the pitch motion is already nondimensional). As the low-frequency motions are relevant only due to their resonant nature, the difference-frequencies of interest are usually the ones that correspond to the natural frequencies of surge, heave and pitch, $\omega_{n 1}, \omega_{n 3}$ and $\omega_{n 5}$.

Since an analytical solution to this problem is unknown, numerical methods are used to solve it. The solution using the slender-body approximation is obtained with METiS, while WAMIT is used to solve the problem with radiation/diffraction theory. Since the numerical solutions take far longer than evaluating the analytical expressions of the bottom mounted cylinder, analyzing a large number of combinations of the relevant dimensionless quantities is not practical. Hence, to narrow down the number of simulations, this section focuses on two particular cases: a deep draft cylinder $(90 \mathrm{~m})$ in deep waters $(150 \mathrm{~m})$ with large natural period of surge $(82 \mathrm{~s})$, which are favorable conditions for Newman's approximation; and a shorter cylinder $(40 \mathrm{~m})$ in shallow waters $(60 \mathrm{~m})$ with a lower natural period of surge (60s), so that Newman's approximation is not expected to perform so well. The same radius of $7.5 \mathrm{~m}$ is considered for both structures, thus the nondimensional draft is considerably reduced from the first case $(\bar{d}=12)$ to the second $(\bar{d}=5.3)$. The natural periods of heave were chosen to be within the range of the simulated waves, so that significant vertical motions can be observed. To ease the distinction between them, the first cylinder will be referred as "long" and the second as "short" (even though it is not actually short, but only shorter than the other), and their properties are given in Table 5.

Table 5 - Properties of the floating cylinders.

\begin{tabular}{lrr}
\hline & Long cyl. & Short cyl. \\
\hline Mass & $16302 \mathrm{t}$ & $7245 \mathrm{t}$ \\
Displacement & $15904 \mathrm{~m}^{3}$ & $7069 \mathrm{~m}^{3}$ \\
Pitch gyradius about center of mass & $40.0 \mathrm{~m}$ & $30.0 \mathrm{~m}$ \\
Radius & $7.5 \mathrm{~m}$ & $7.5 \mathrm{~m}$ \\
Draft & $90.0 \mathrm{~m}$ & $40.0 \mathrm{~m}$ \\
KG & $34.5 \mathrm{~m}$ & $15.0 \mathrm{~m}$ \\
KB & $45.0 \mathrm{~m}$ & $20.0 \mathrm{~m}$ \\
BM & $0.2 \mathrm{~m}$ & $0.4 \mathrm{~m}$ \\
GM & $10.7 \mathrm{~m}$ & $5.4 \mathrm{~m}$ \\
Water depth & $150 \mathrm{~m}$ & $60 \mathrm{~m}$ \\
\hline Surge natural period & $82.0 \mathrm{~s}$ & $60.0 \mathrm{~s}$ \\
Heave natural period & $19.5 \mathrm{~s}$ & $13.4 \mathrm{~s}$ \\
Pitch natural period & $30.0 \mathrm{~s}$ & $28.0 \mathrm{~s}$ \\
\hline Nondimensional draft $(\bar{d})$ & 12.0 & 5.3 \\
Nondimensional water depth $(\bar{h})$ & 20.0 & 8.0 \\
Nondimensional surge natural frequency $\left(\bar{\omega}_{n, 1}\right)$ & 0.300 & 0.259 \\
Nondimensional heave natural frequency $\left(\bar{\omega}_{n, 3}\right)$ & 1.258 & 1.157 \\
Nondimensional pitch natural frequency $\left(\bar{\omega}_{n, 5}\right)$ & 0.827 & 0.555 \\
\hline
\end{tabular}

Only the loads acting at the difference frequencies of surge and pitch are analyzed, 
since no significant slow heave was observed in the conditions that were analyzed, and the simulated waves corresponding to each cylinder are specified in Tables 6 and 7. The difference in the quantity of simulated waves is due to removing pairs with wave periods above $25 \mathrm{~s}$, which is already a significantly large value.

Table 6 - List of the bichromatic waves simulated for the long cylinder for each $\omega_{j l}^{-}$. Each component has an amplitude of $1 \mathrm{~m}$.

\begin{tabular}{l|cccc|cccc}
\hline & \multicolumn{5}{|c|}{$\bar{\omega}_{j l}^{-}=0.300$} & \multicolumn{5}{c}{$\bar{\omega}_{j l}^{-}=0.827$} \\
& $T_{j}$ & $T_{l}$ & $\lambda_{j} / D$ & $\lambda_{l} / D$ & $T_{j}$ & $T_{l}$ & $\lambda_{j} / D$ & $\lambda_{l} / D$ \\
\hline BIC01 & 2.2 & 2.3 & 0.5 & 0.5 & 2.2 & 2.4 & 0.5 & 0.6 \\
BIC02 & 4.4 & 4.7 & 2.1 & 2.3 & 4.4 & 5.2 & 2.1 & 2.8 \\
BIC03 & 5.9 & 6.3 & 3.6 & 4.2 & 5.9 & 7.3 & 3.6 & 5.6 \\
BIC04 & 7.0 & 7.7 & 5.2 & 6.2 & 7.0 & 9.2 & 5.2 & 8.8 \\
BIC05 & 8.0 & 8.9 & 6.7 & 8.2 & 8.0 & 11.0 & 6.7 & 12.5 \\
BIC06 & 8.9 & 10.0 & 8.3 & 10.4 & 8.9 & 12.7 & 8.3 & 16.7 \\
BIC07 & 9.7 & 11.0 & 9.8 & 12.6 & 9.7 & 14.4 & 9.8 & 21.3 \\
BIC08 & 10.5 & 12.0 & 11.4 & 14.9 & 10.5 & 16.0 & 11.4 & 26.3 \\
BIC09 & 11.1 & 12.9 & 12.9 & 17.3 & 11.1 & 17.7 & 12.9 & 31.5 \\
BIC10 & 11.8 & 13.8 & 14.5 & 19.7 & 11.8 & 19.4 & 14.5 & 36.8 \\
BIC11 & 12.4 & 14.6 & 16.0 & 22.1 & 12.4 & 21.2 & 16.0 & 42.2 \\
BIC12 & 13.0 & 15.5 & 17.6 & 24.6 & 13.0 & 23.0 & 17.6 & 47.6 \\
BIC13 & 13.6 & 16.3 & 19.1 & 27.0 & 13.6 & 24.8 & 19.1 & 53.1 \\
BIC14 & 14.1 & 17.1 & 20.7 & 29.5 & - & - & - & - \\
BIC15 & 14.7 & 17.9 & 22.2 & 31.9 & - & - & - & - \\
BIC16 & 15.2 & 18.7 & 23.8 & 34.4 & - & - & - & - \\
BIC17 & 15.7 & 19.4 & 25.3 & 36.8 & - & - & - & - \\
BIC18 & 16.2 & 20.2 & 26.9 & 39.3 & - & - & - & - \\
BIC19 & 16.7 & 21.0 & 28.4 & 41.7 & - & - & - & - \\
BIC20 & 17.2 & 21.8 & 30.0 & 44.1 & - & - & - & - \\
BIC21 & 18.3 & 23.6 & 33.3 & 49.4 & - & - & - & - \\
\hline & & & & & & & &
\end{tabular}

\subsubsection{Numerical models}

For conciseness, some of the procedures will be illustrated for the short cylinder only, as they were the same that were employed to model the long cylinder.

\subsubsection{WAMIT}

WAMIT simulations were performed with version $6.106 \mathrm{~S}$ of the software, which is capable of doing complete second-order hydrodynamic analyses, such as the calculation of sum- and 
Table 7 - List of the bichromatic waves simulated for the short cylinder for each $\omega_{j l}^{-}$. Each component has an amplitude of $1 \mathrm{~m}$.

\begin{tabular}{l|cccc|cccc}
\hline & \multicolumn{5}{|c|}{$\bar{\omega}_{j l}^{-}=0.260$} & \multicolumn{5}{c}{$\bar{\omega}_{j l}^{-}=0.555$} \\
& $T_{j}$ & $T_{l}$ & $\lambda_{j} / D$ & $\lambda_{l} / D$ & $T_{j}$ & $T_{l}$ & $\lambda_{j} / D$ & $\lambda_{l} / D$ \\
\hline BIC01 & 2.2 & 2.3 & 0.5 & 0.5 & 2.2 & 2.4 & 0.5 & 0.6 \\
BIC02 & 4.4 & 4.8 & 2.1 & 2.4 & 4.4 & 5.3 & 2.1 & 2.9 \\
BIC03 & 5.9 & 6.5 & 3.6 & 4.4 & 5.9 & 7.5 & 3.6 & 5.8 \\
BIC04 & 7.0 & 8.0 & 5.2 & 6.6 & 7.0 & 9.4 & 5.2 & 9.1 \\
BIC05 & 8.0 & 9.3 & 6.7 & 8.9 & 8.0 & 11.3 & 6.7 & 12.7 \\
BIC06 & 8.9 & 10.5 & 8.3 & 11.2 & 8.9 & 13.1 & 8.3 & 16.3 \\
BIC07 & 9.8 & 11.7 & 9.8 & 13.5 & 9.8 & 15.0 & 9.8 & 19.9 \\
BIC08 & 10.6 & 12.8 & 11.4 & 15.8 & 10.6 & 17.0 & 11.4 & 23.7 \\
BIC09 & 11.4 & 14.0 & 12.9 & 18.1 & 11.4 & 19.1 & 12.9 & 27.6 \\
BIC10 & 12.2 & 15.3 & 14.5 & 20.4 & 12.2 & 21.5 & 14.5 & 31.7 \\
BIC11 & 13.0 & 16.5 & 16.0 & 22.8 & 13.0 & 24.1 & 16.0 & 36.3 \\
BIC12 & 13.8 & 17.9 & 17.6 & 25.2 & - & - & - & - \\
BIC13 & 14.6 & 19.3 & 19.1 & 27.7 & - & - & - & - \\
BIC14 & 15.4 & 20.7 & 20.7 & 30.3 & - & - & - & - \\
BIC15 & 16.2 & 22.2 & 22.2 & 33.0 & - & - & - & - \\
BIC16 & 17.1 & 23.9 & 23.8 & 35.8 & - & - & - & - \\
\hline
\end{tabular}

difference-frequency QTFs, second-order RAOs, among other quantities. The submerged part of the hull was represented by a higher-order mesh, meaning that the body is described by B-splines and quantites are continuous inside each patch (WAMIT, 2004). Due to the symmetry of the problem, only one quadrant needs to be discretized, as illustrated in Figure 18. Only two patches were actually needed, but an additional one was used to discretize the interior free surface in order to remove irregular frequencies (ZHU, 1994).

The higher-order method allows a straightforward automatic subdivision of the patches into panels by specifying their approximate maximum length via a panel size parameter. Its value was chosen based on a convergence analysis that considered three different meshes: a coarse one, with panel size 5.0; a medium mesh, with panel size 2.5; and a fine one with panel size 1.25, as illustrated in Figure 19. The results show that differences between the fine and medium mesh can still be observed for very short waves, but as only the BIC01 wave is inside this range, the panel size equal to 2.5 was considered good enough. This value was adopted in all the results presented ahead.

The potential damping of a vertical cylinder such as the one analyzed here is very small. For instance, the term $B_{33}$ obtained with WAMIT for the short cylinder corresponds to less than $0.5 \%$ of the critical heave damping, which leads to very large and unrealistic 
Figure 18 - Higher-order mesh of the cylinder with $\bar{d}=5.3$.

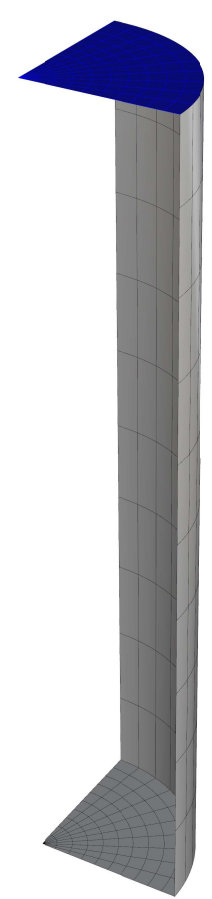

Figure 19 - Mean surge force acting on the cylinder with $\bar{d}=5.3$ for different panel sizes.

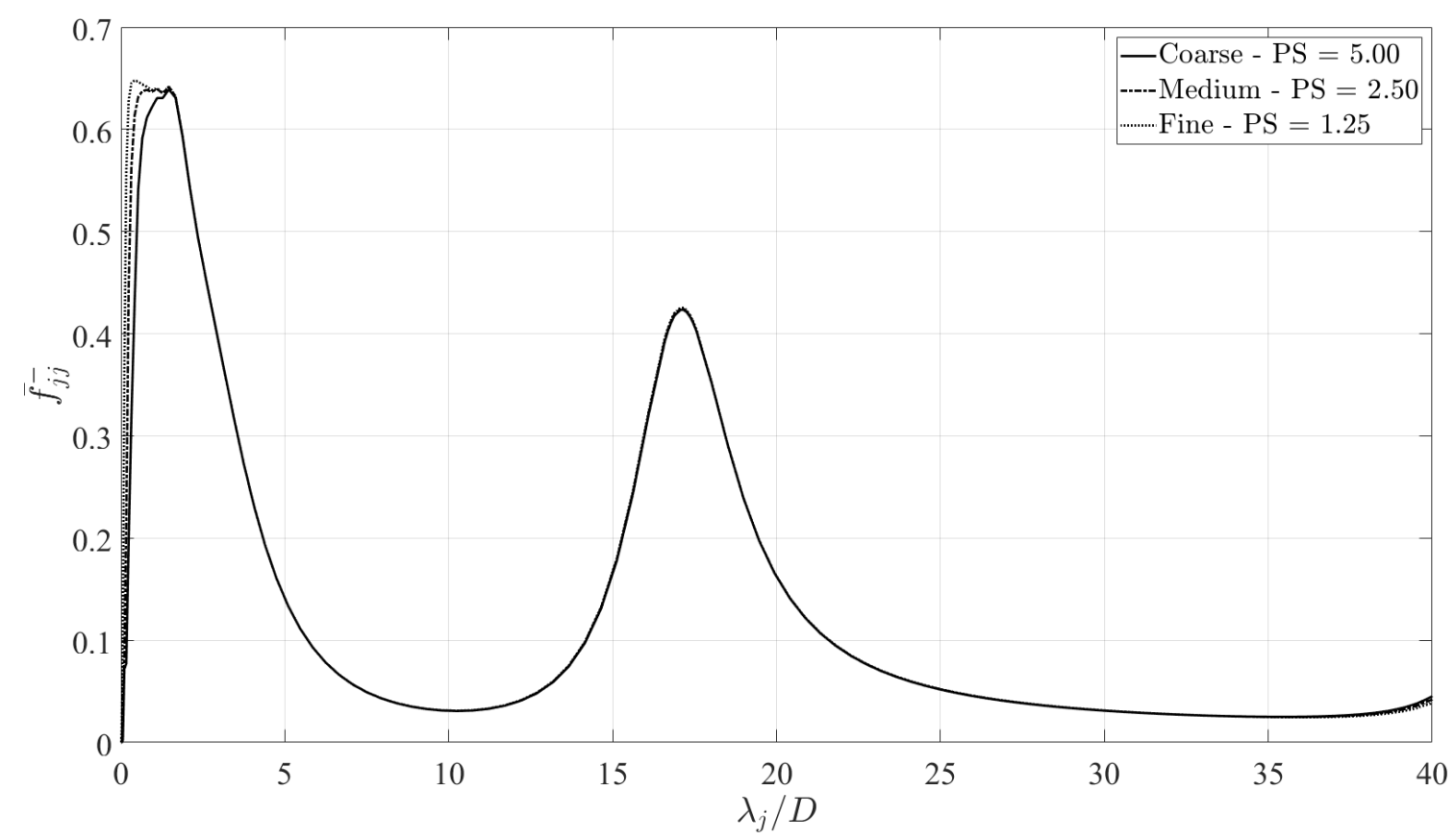

motions close to the natural frequency. This is due to neglecting viscous damping, which can be included in WAMIT by an external linear damping matrix. As only heave and pitch have natural frequencies within the range excited by first-order loads, only the terms $B_{33}^{\text {ext }}$ and $B_{55}^{\text {ext }}$ are considered. They were set in order to approximately match the motion RAOs obtained with METiS, which considers a drag coefficient $C_{D}=1.0$ and an axial drag coefficient $C_{D z}=4.0$ for both cylinders. As evidenced by the results presented in Section 5.2.2, this is achieved by considering the values given in Table 8 in WAMIT. 
Table 8 - External linear heave and pitch damping considered in the WAMIT simulations.

\begin{tabular}{|c|c|c|c|c|}
\hline & & External damping & Critical damping & Damping level \\
\hline \multirow{2}{*}{$\frac{\overrightarrow{0}}{\tilde{G}}$} & Heave (N.s/m) & $5.08 \mathrm{E}+05$ & $6.78 \mathrm{E}+06$ & $7.5 \%$ \\
\hline & Pitch (N.m.s/rad) & $8.47 \mathrm{E}+08$ & $3.39 \mathrm{E}+09$ & $2.5 \%$ \\
\hline \multirow{2}{*}{ 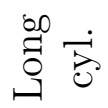 } & Heave (N.s/m) & $5.12 \mathrm{E}+05$ & $1.11 \mathrm{E}+07$ & $4.6 \%$ \\
\hline & Pitch (N.m.s/rad) & $5.53 \mathrm{E}+08$ & $1.60 \mathrm{E}+10$ & $3.5 \%$ \\
\hline
\end{tabular}

The second-order force QTFs were calculated considering the influence of the second-order potential, but without the free-surface forcing terms in the second-order problem. This is a very useful approximation because it allows the problem to be solved without meshing the free surface, and it is justified based on past works that found out that these terms are negligible for similar structures (KIM, 1992; MOLIN; CHEN, 2002; SIMOS; RUGGERI, et al., 2018). The influence of the free-surface forcing terms for a more complex structure composed of four vertical cylinders is analyzed in Section 6.3.1, with the same conclusion that they are unimportant.

\subsubsection{METiS}

The cylinders were modeled in METiS considering a drag coefficient of $C_{D}=1.0$, which is within the range of values adopted in the literature (SARPKAYA, 2010), and an axial drag coefficient $C_{D z}=4.0$, which was established in order to keep the heave motions within reasonable limits. This criterion for setting $C_{D z}$ is acceptable because this test case is used simply to verify the slender-body approach and not to reproduce a real structure, in such a way that the most important is to guarantee that the first-order motions obtained with METiS and WAMIT are similar.

Since the added mass terms of the slender-body approximation neglect threedimensional effects, the added mass coefficient $C_{a}$ is constant along all the length of the cylinder. Thus, divergences with respect to the results obtained with WAMIT are expected, specially concerning the pitch added mass and the short cylinder. Considering that a reasonable comparison of the second-order loads demands that first-order results be similar, it is reasonable to impose that the natural periods of motion obtained with both software be the same. Besides, the second-order loads are only relevant because they excite the natural frequencies of motion, which further emphasizes the importance of properly matching these frequencies.

This is achieved for the surge motion by setting the added mass coefficients to $C_{a}=0.89$ for the short cylinder and $C_{a}=0.95$ for the long cylinder, while the natural period of heave requires $C_{a z}=0.48$ for both of them. As illustrated by Figure 20, the added mass coefficient needed to match the added mass calculated with WAMIT would actually be a frequency-dependent quantity, but this effect is lost in the slender-body 
Figure 20 - Added mass coefficients needed to match the added mass terms computed with WAMIT for the long (left) and short (right) cylinders.
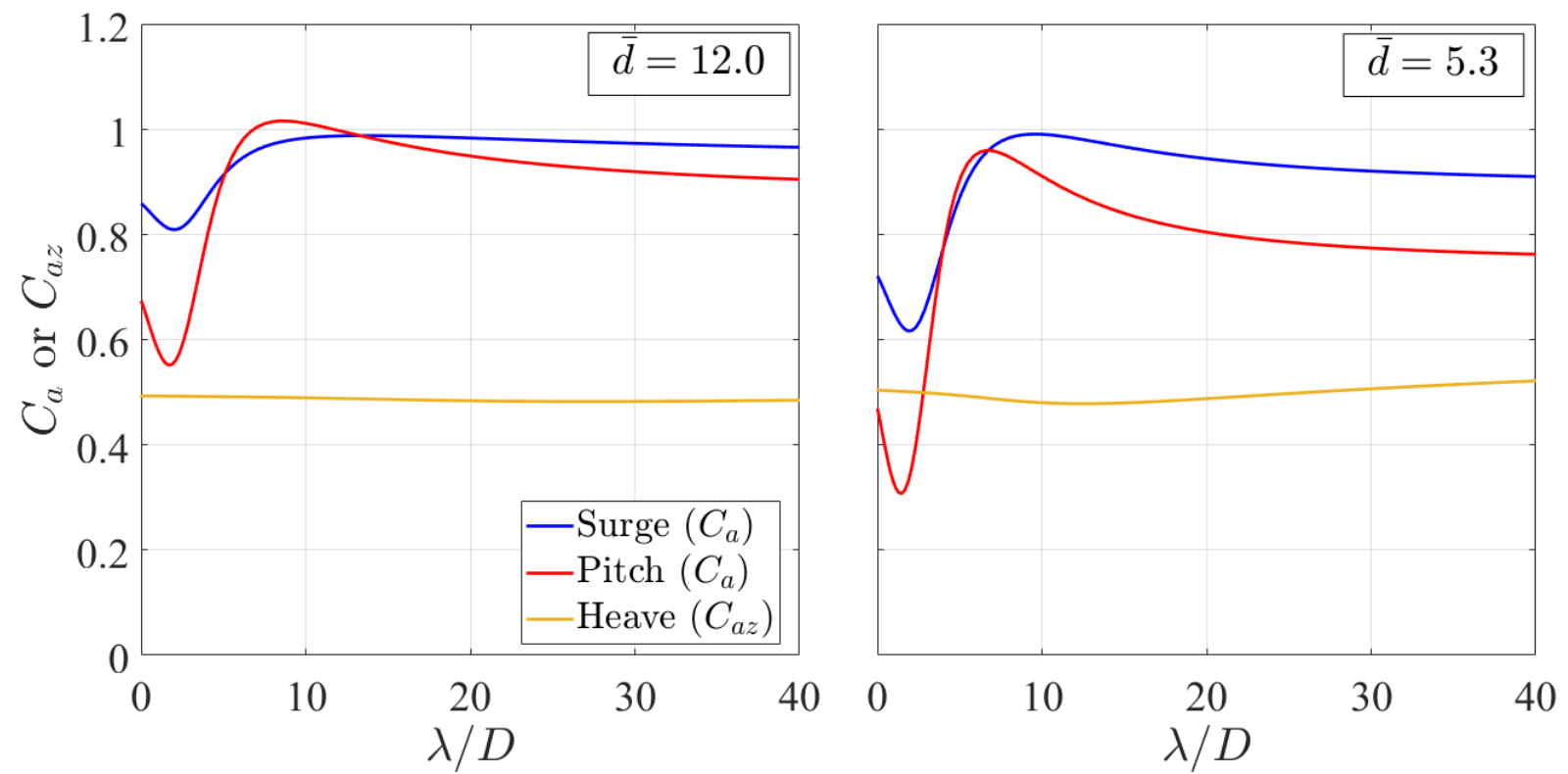

method when radiation effects were neglected. The graph also shows that the term $A_{55}$ of the added mass matrix is not well reproduced, a consequence of adopting a constant $C_{a}$ along the whole cylinder span, as already pointed out. For the long cylinder, the difference is about $7 \%$ at the natural frequency of pitch, but it is around $30 \%$ for the short cylinder. Nevertheless, these discrepancies induce small errors in the natural period of pitch because the moment of inertia $I_{y y}$ of the body is far larger than $A_{55}$. Indeed, since the natural periods provided in Table 5 were calculated with the slender-body approach, the difference in $A_{55}$ causes the natural pitch period obtained with WAMIT to be $0.7 \mathrm{~s}$ larger for the long cylinder (corresponding to a difference of 2.5\%), while for the short cylinder it is $0.28 \mathrm{~s}$ larger (difference of about $1.0 \%$ ). In any case, since the natural period of pitch is outside the range of the waves analyzed, this effect does not have a significant impact on the results.

In order to keep the excursions of the cylinder within reasonable limits and to obtain the desired natural frequency of surge, an external stiffness term $K_{11}$ was adopted to emulate the action of the mooring system, with the following values for each of the cylinders:

$$
K_{11}= \begin{cases}1.50 \mathrm{e} 5 \mathrm{~N} / m & \text { for the short cylinder } \\ 1.86 \mathrm{e} 5 \mathrm{~N} / m & \text { for the long cylinder }\end{cases}
$$

and without any additional stiffness in the other degrees of freedom.

Two different convergence analysis were performed to set the numerical parameters of the METiS model. The first was to establish the number of sections for the numerical 
integration along the length of the cylinder, done so by comparing one of the secondorder force components with its analytical solution. In fact, as the cylinder adopted in this analysis is vertical, most of the forces presented in Section 3.6 could be integrated analytically, but they are integrated numerically in order to verify the numerical tool. As illustrated by Figure 21, which presents the force due to the convective acceleration acting on the short cylinder for the BIC01 wave and $\bar{\omega}_{j l}=0.555$, using cylinder sections of $0.50 \mathrm{~m}$ is enough, as the difference with respect to a finer discretization and to the analytical solution are negligible. This value of $\Delta z$ was adopted for both the short and the long cylinder.

Figure 21 - Horizontal force due to the convective acceleration calculated with METiS for the short cylinder under the action of the BIC01 wave and $\bar{\omega}_{j l}=0.555$ for different discretizations.

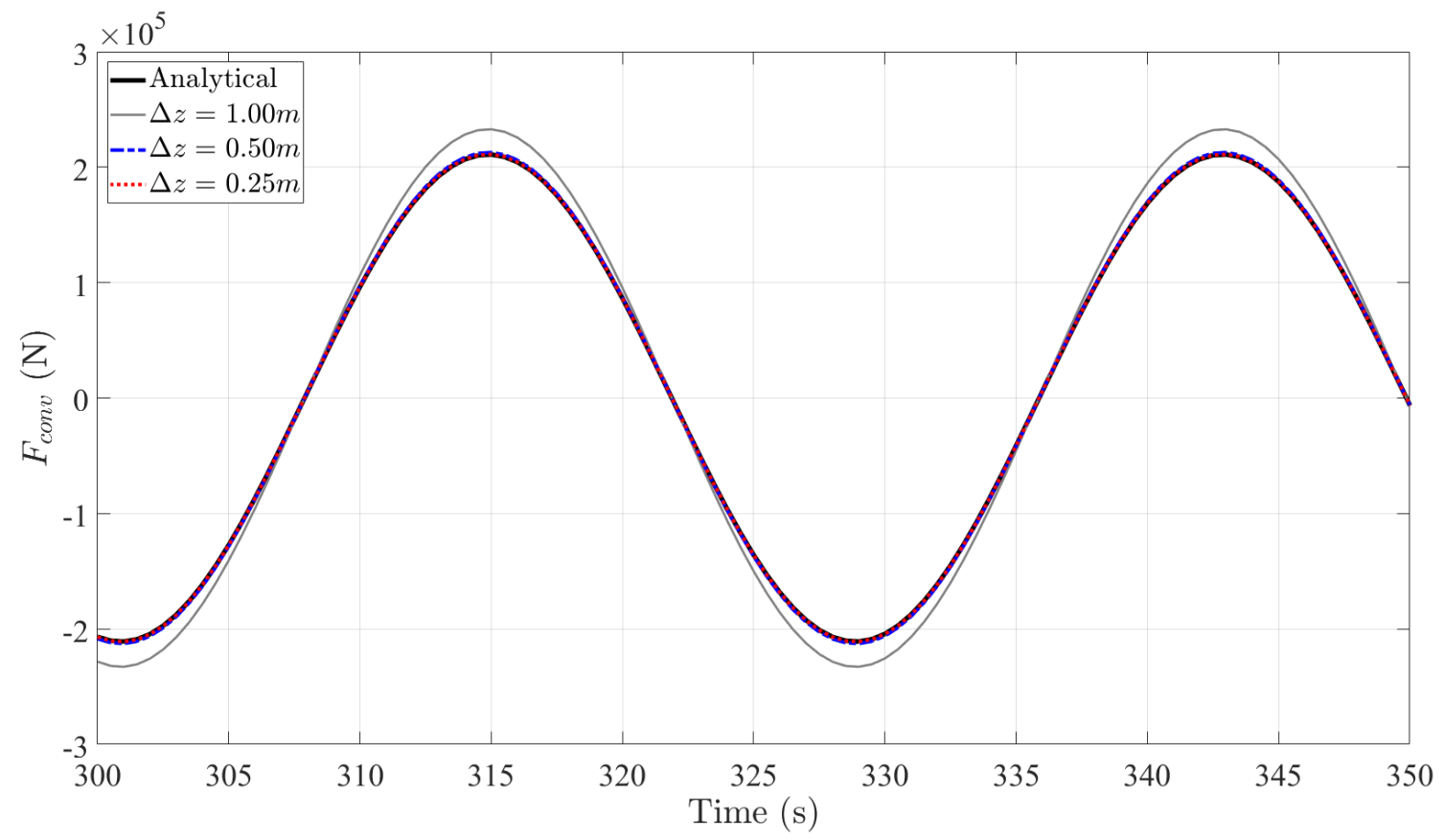

The second convergence analysis concerns the time step, which was established by analyzing the test case with the fastest dynamics, namely the short cylinder for the BIC01 wave and $\bar{\omega}_{j l}=0.555$. Three different time steps were verified, as presented in Figure 22, and since the differences between a time step of $0.10 \mathrm{~s}$ and $0.05 \mathrm{~s}$ were very small, the value $\Delta t=0.10 \mathrm{~s}$ was deemed good enough.

Since METiS is a time-domain software, each wave condition corresponds to a different simulation that results in time series of forces and motions. For each of the bichromatic waves, these time series are composed of components acting at three different frequencies: the ones of the two incoming waves, $\omega_{1}$ and $\omega_{2}$, which are due to the first-order wave forces; and the difference frequency, $\omega^{-}=\omega_{1}-\omega_{2}$, associated to the second-order wave forces. As these frequencies are known, the complex amplitude of a component acting at a given frequency $\hat{\omega}, \Gamma(\hat{\omega})$, can be obtained by projecting the signal $\gamma(t)$ onto a sine 
Figure 22 - Surge motion of the short cylinder for the BIC01 wave and $\bar{\omega}_{j l}=0.555$ for different time steps.

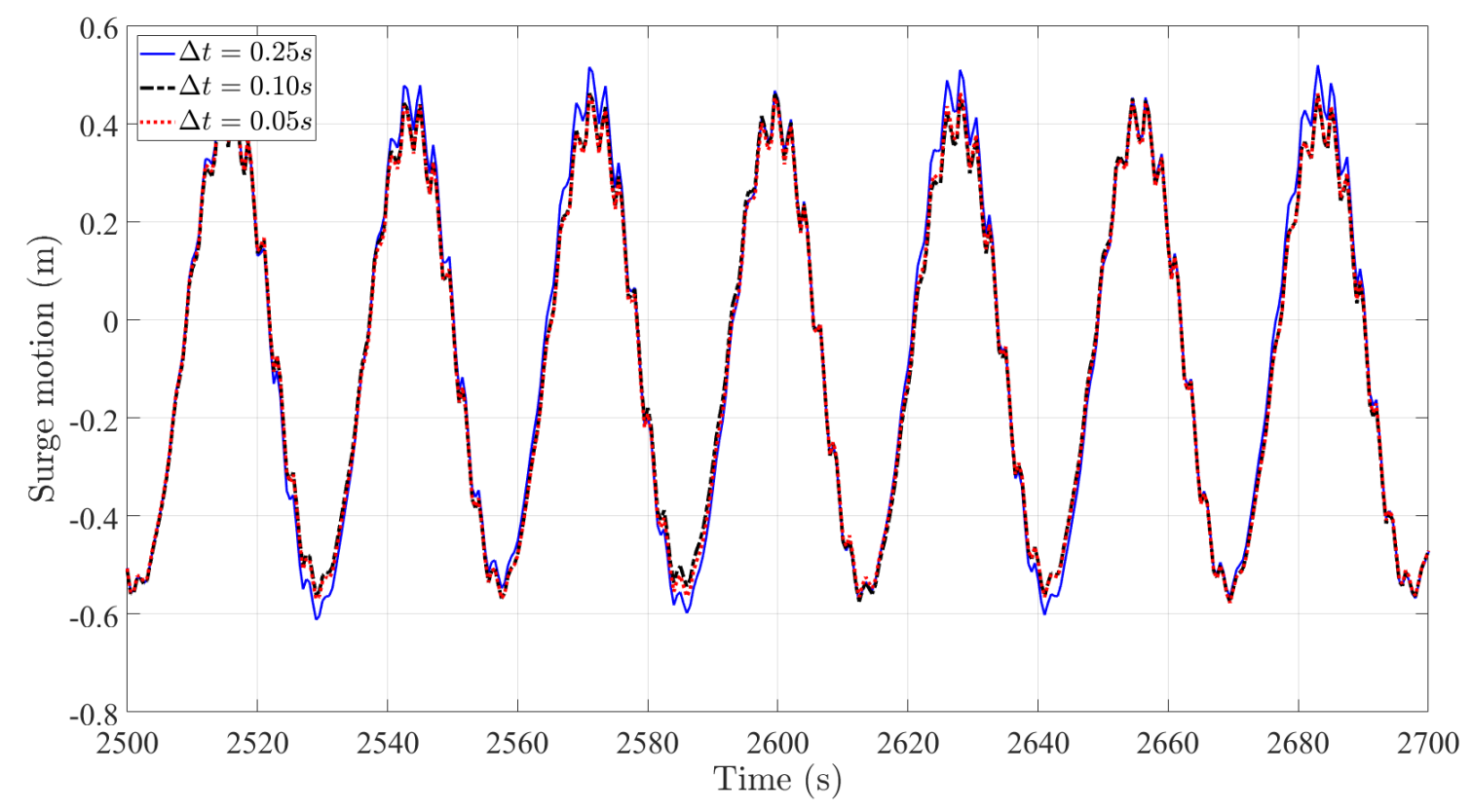

and a cosine functions:

$$
\begin{aligned}
& \Re\{\Gamma(\hat{\omega})\}=\frac{2}{T_{1}-T_{0}} \int_{T_{0}}^{T_{1}} \gamma(t) \cos (\hat{\omega} t) \mathrm{d} t \\
& \Im\{\Gamma(\hat{\omega})\}=-\frac{2}{T_{1}-T_{0}} \int_{T_{0}}^{T_{1}} \gamma(t) \sin (\hat{\omega} t) \mathrm{d} t
\end{aligned}
$$

which is quite convenient because both the amplitude of motion and the phase with respect to the incoming wave can be readily evaluated from the complex amplitude, allowing a straightforward comparison with results computed in frequency-domain with WAMIT. Ideally, $T_{0}$ and $T_{1}$ would encompass an integer number of cycles with frequency $\hat{\omega}$, but this is rather impractical. Instead, good results can be obtained by considering a large number of cycles, in such a way that the error is diluted. To do so, the simulations were performed for a total simulation time of $3000 \mathrm{~s}$ with a time ramp of $200 \mathrm{~s}$ (see Equation 4.19), which was more than enough to achieve a steady-state response, and only the last half of the signal was used to compute the amplitudes in order to avoid the transient response to affect the results. Since this procedure provides the force acting at a given frequency, the result was divided by two in order to be directly compared with the QTF values computed with WAMIT, as it would be the sum of the components acting at the frequencies $\omega_{j l}^{-}$and $\omega_{l j}^{-}$that are output by the latter. 


\subsubsection{First-order forces and motions}

Figures 23 and 24 present, respectively, the nondimensional first-order wave loads and the corresponding motion RAOs calculated with WAMIT and METiS.

Regarding the surge force and pitch moment, the behavior is quite similar to the one previously observed for the second-order loads acting on the bottom mounted cylinder, i.e. the slender-body approximation matches very well the imaginary part of the force/moment, except for short waves, but it predicts a null real part of the loads (except for numerical errors in the process of evaluating the complex amplitude) for any wave frequency, which is only acceptable in long waves. For $\lambda / D>5$, which is the threshold commonly adopted for the validity of Morison's equation, the amplitude of the surge and pitch loads predicted with both software are within $5 \%$ of each other.

Concerning the heave loads, the opposite is observed, with METiS being able to reproduce the real part of the force instead of the imaginary part, a consequence of the $90^{\circ}$ phase shift between the horizontal acceleration and the fluid pressure. In this case the agreement is even better, as the loads acting at short waves are negligible due to the exponential decay of the fluid pressure. Nevertheless, this behavior is dependent on the relation between the wave length and the draft of the cylinder, $\lambda / d$, which is not analyzed in details here, and different conclusions might be drawn for a shallow draft structure.

Given the differences for the loads in short waves, it is indeed remarkable that the first-order RAOs match quite well for all the wave range. The reason is that the dynamics of the body in short waves (thus high frequency) is dominated by inertial effects, in such a way that the overestimation of the first-order load is compensated by an overestimation of the added mass. In other words, the fact that the added mass factor $C_{a}$ is not corrected to account for wave scattering (using, for instance, the formulation due to MacCamy and Fuchs (1954)) impacts similarly both the forcing and the inertia of the dynamic system.

Due to this good agreement, it is possible to compare the second-order loads in the next section bearing in mind that occasional differences are due to the force models, and not to discrepancies on the first-order motions that feed them.

\subsubsection{Second-order forces}

The nondimensional second-order horizontal forces calculated with WAMIT, METiS and Newman's approximation are compared in Figures 25 and 26, with the forces calculated for a difference-frequency corresponding to the natural frequency of surge and the results given as a function of the nondimensional length of the shortest wave of the pair. In order to evidence the effects due to body motions, results considering the body fixed are also included in the graphs. As expected, the slender-body approximation performs poorly in short waves, but there is a general good agreement, with some remarks, for $\lambda_{j} / D>10$, 
Figure 23 - First-order nondimensional surge (top), heave (middle) and pitch (bottom) loads for the long (left) and short (right) cylinders.
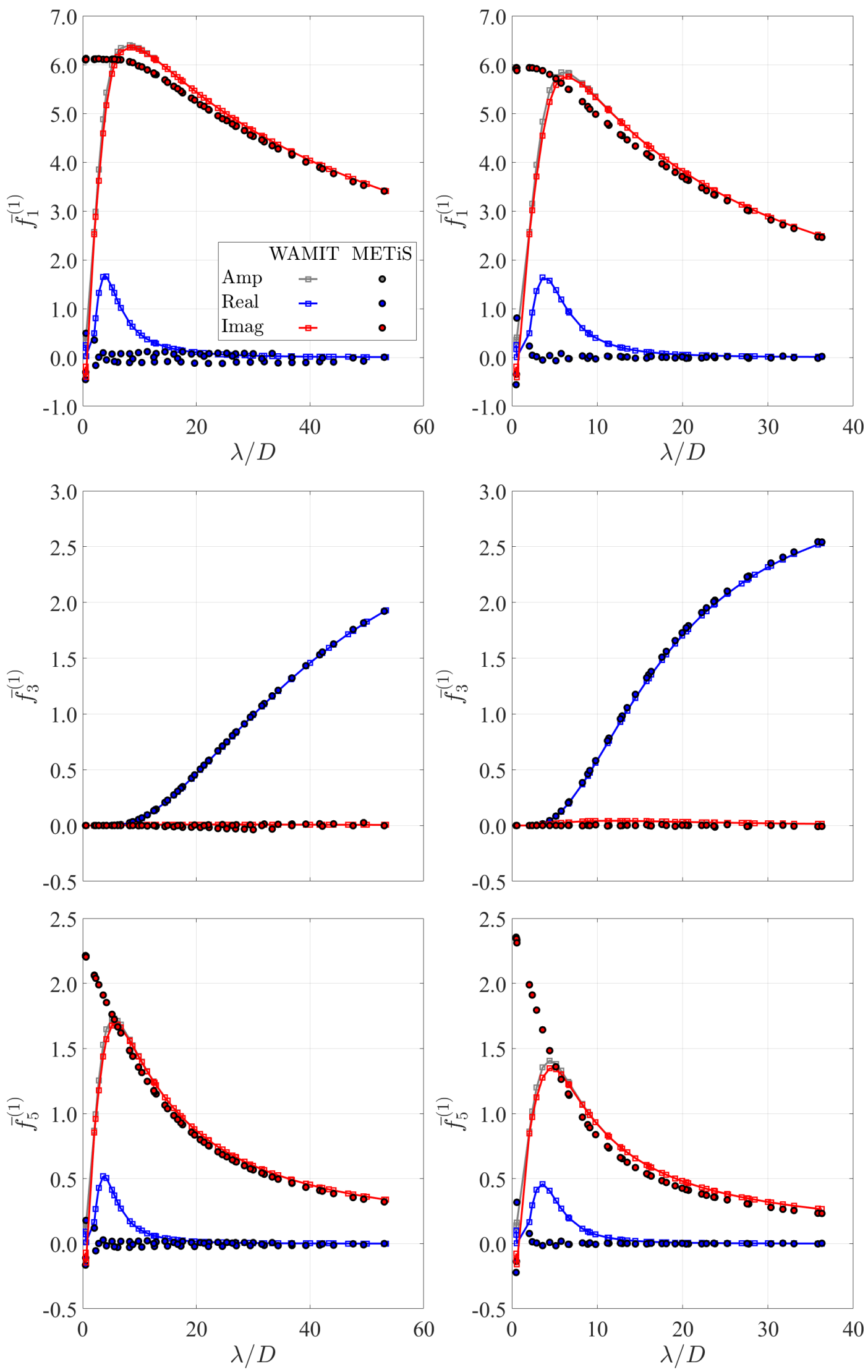
Figure 24 - Surge (top), heave (middle) and pitch (bottom) RAOs for the long (left) and short (right) cylinders.
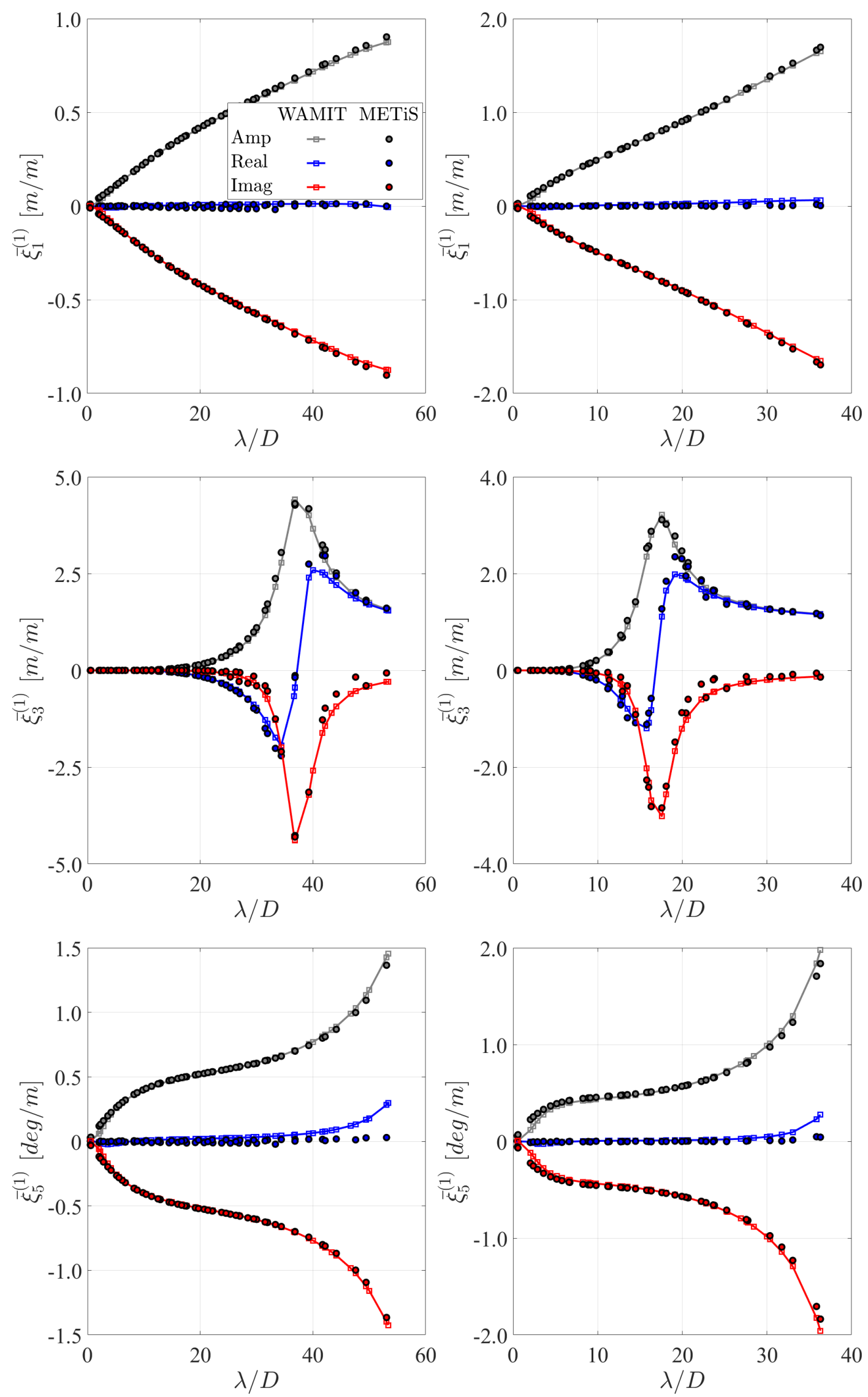
which is the threshold indicated by Rainey (1995) for the validity of his formulation.

Figure 25 - Amplitude of the nondimensional second-order horizontal force acting on the long (left $\left.-\bar{\omega}_{j l}^{-}=0.300\right)$ and short (right $\left.-\bar{\omega}_{j l}^{-}=0.259\right)$ cylinders calculated with and without first-order motions as a function of the length of the shortest wave of the pair.
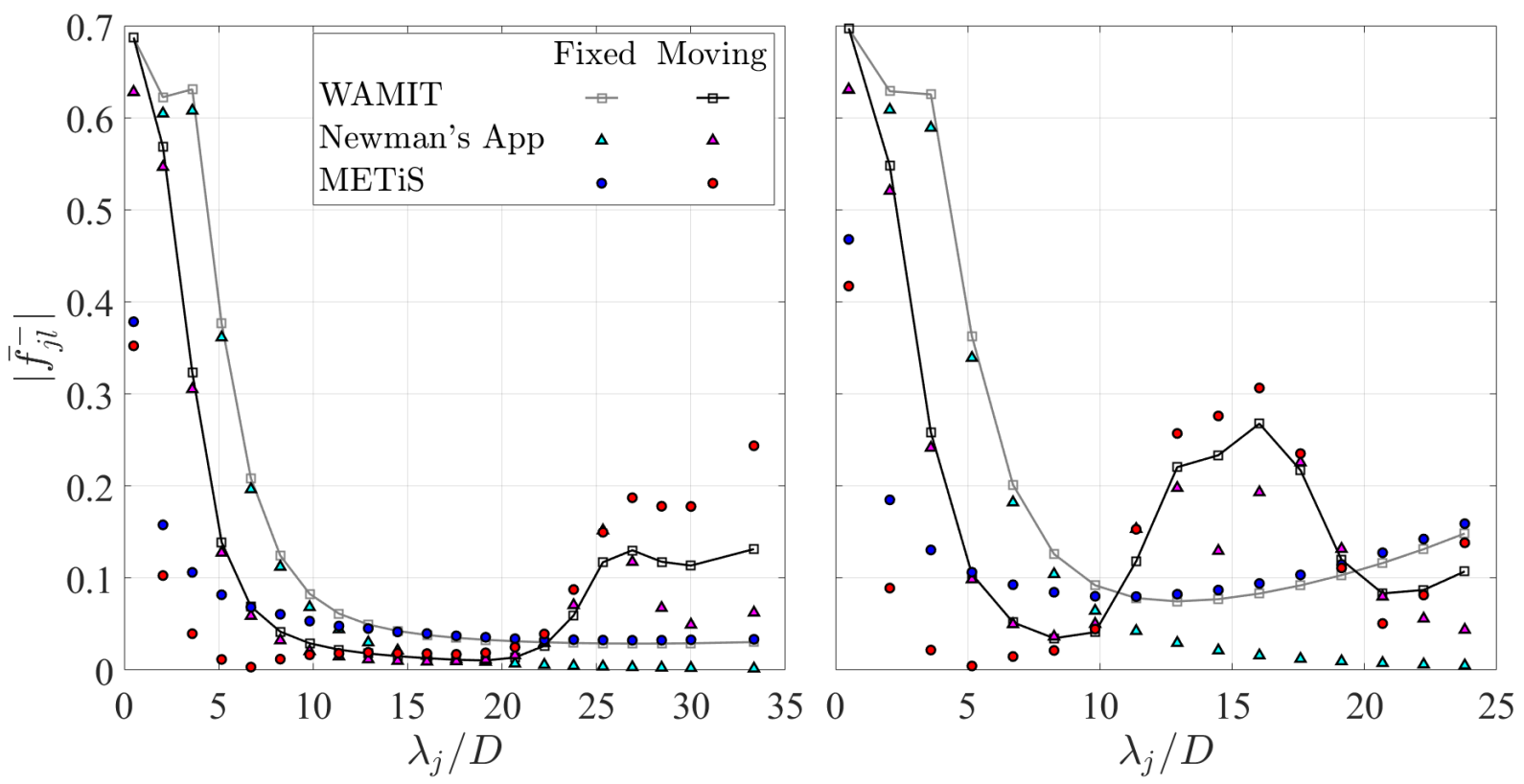

Figure 26 - Real and imaginary parts of the nondimensional second-order horizontal force acting on the long (left $-\bar{\omega}_{j l}^{-}=0.300$ ) and short (right $-\bar{\omega}_{j l}^{-}=0.259$ ) cylinders as a function of the length of the shortest wave of the pair.
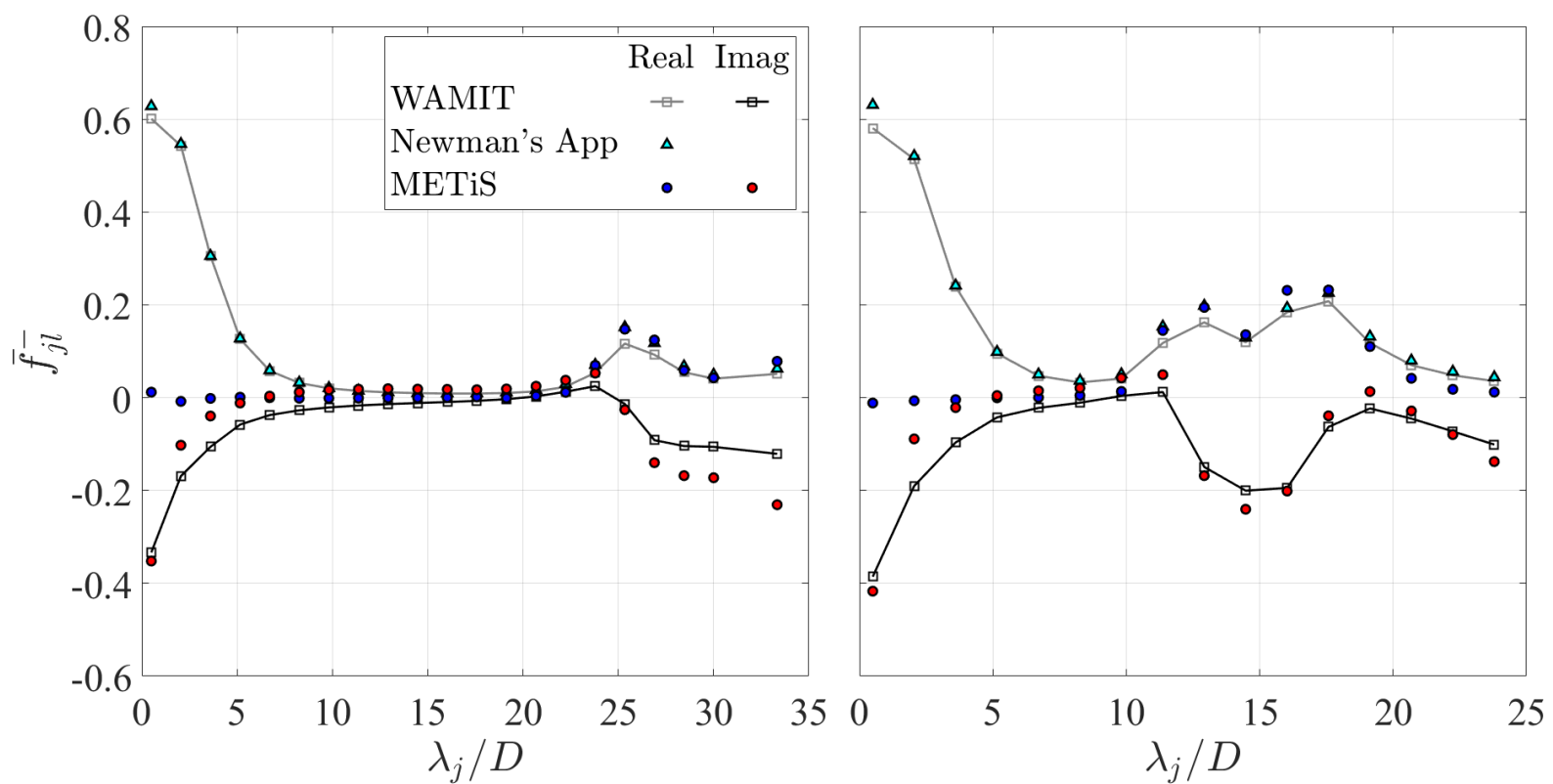

Regarding the long cylinder, however, Figure 26 shows that the imaginary part of the force calculated with METiS presents a small offset for a wave range roughly between $\lambda_{j} / D=10$ and $\lambda_{j} / D=25$, while the results for the short cylinder have a better agreement, 
which may seen unexpected at first sight. The explanation comes from inspecting the force components computed with the slender-body approximation, given in Figure 27. Since this issue is presented by the long cylinder even in waves for which the heave motion is very small $\left(\lambda_{j} / D<15\right)$, in such a way that almost all of the force components are basically the same as for the fixed cylinder, which, on its turn, shows a good agreement in this wave range, it must come from the only exception, namely the force due to the acceleration gradient, $\mathbf{F}_{\mathbf{X} \cdot \nabla}^{(2)}$ (the equivalent of component IV from Pinkster (1979), see Section 3.6). For $\lambda_{j} / D<15, \mathbf{F}_{\mathbf{X} \cdot \nabla}^{(2)}$ is due to the horizontal displacement of the points along the cylinder axis (as the vertical displacement is negligible), and this seems to be the source of this discrepancy.

Though the short cylinder has the same problem regarding $\mathbf{F}_{\mathbf{X} \cdot \nabla}^{(2)}$, it is much less relevant than for the long cylinder because heave motion, whose effects seem to be well modeled by the slender-body approximation, is relevant for a significant part of the range of the wave lengths that presented this issue (the natural periods of heave, which is caused simply by the pressure integration at the bottom surface, correspond to $\lambda / D \approx 37$ for the long cylinder and $\lambda / D \approx 17$ for the short one). Besides, as the cylinder is shorter, the pitch motion induces smaller displacements along the cylinder, reducing the influence of $\mathbf{F}_{\mathbf{X} \cdot \nabla}^{(2)}$ in the total force. These results show that, once body motions are included, it is not a simple task to determine the conditions in which the slender-body approximation presented in this thesis is acceptable, since different effects seem to follow distinct thresholds in terms of $\lambda_{j} / D$ and of the other dimensionless quantities that describe the problem.

Concerning Newman's approximation, it reproduces the force calculated with WAMIT for the short cylinder quite well, except close to a peak of the imaginary part of the force that corresponds to frequency pairs that match the natural frequency of heave. For the long cylinder, it performs very well except in very long waves, for which it underestimates the horizontal load due to neglecting the imaginary part of the force; on the other hand, the slender-body approximation overestimates the force in the same conditions.

As the forces obtained with the slender-body approximation have a non-null real part, it is no longer possible to directly sum the results with the ones obtained with Newman's approximation, as part of the effects would be computed twice. Nevertheless, the results in Figure 26 apparently indicate that it still would be possible to sum Newman's approximation with the imaginary part of the force calculated with the slender-body approximation. However, it is not clear how to do that in time domain, but some suggestions on possible ways of exploiting this complementarity between these two approximations are given in Chapter 7.

It is noteworthy the relevance of the force due to the rotation term from Rainey's formulation, $\mathbf{F}_{\mathrm{rslb}}^{(2)}$, as illustrated in Figure 27. It was already shown in the analysis of the 
Figure 27 - Amplitude of the components of the nondimensional second-order horizontal force acting on the long (left $\left.-\bar{\omega}_{j l}^{-}=0.300\right)$ and short (right $-\bar{\omega}_{j l}^{-}=0.259$ ) cylinders calculated with METiS as a function of the length of the shortest wave of the pair. The definition of each component is given in Section 3.6.
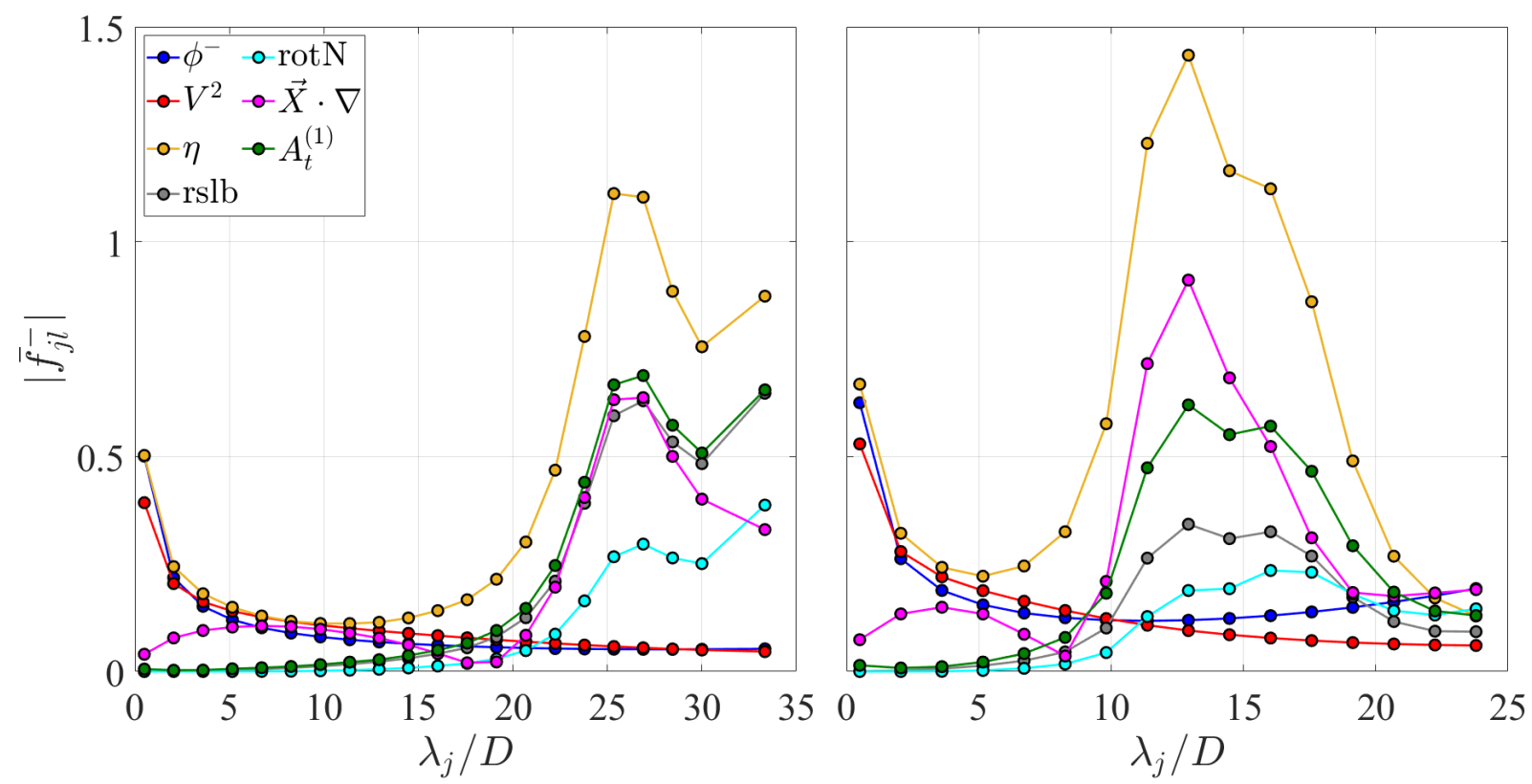

bottom-mounted cylinder that the force due to axial-divergence acceleration, $\mathbf{F}_{\text {axdv }}^{(2)}$, needs to be added to the one due to the convective acceleration, $\mathbf{F}_{\text {conv }}^{(2)}$, in order to match the force component that results from the pressure drop due to the quadratic velocity term of Bernoulli's equation (force component II from Pinkster (1979)). Hence, the common practice of analyzing second-order loads by simply applying Morison's equation with secondorder wave kinematics is not strictly correct, and the additional terms from Rainey's formulation should not be disregarded before verifying their impact on the results.

Finally, Figure 28 compares the amplitudes of the second-order moment with respect to the mean water line, but this time for the difference-frequency corresponding to the natural frequency of pitch of each cylinder. Though the results obtained with METiS follow the same trend as the ones computed with WAMIT, the agreement is not as good as the ones observed for the horizontal force, with discrepancies that are comparable to the ones obtained with Newman's approximation. The reason for this is that end effects, both related to the incoming flow and to the first-order pitch motion, are more relevant for the moment than for the force, as they are magnified by the lever arm.

As both the vertical acceleration and the pressure have a $90^{\circ}$ phase with respect to the horizontal acceleration, it follows that the vertical force predicted with the slenderbody approximation has a real amplitude when the body is not allowed to move, in opposition to the purely imaginary amplitude of the horizontal force. The same conclusion is presented by Molin and Chen (2002) for a horizontal cylinder, which is a case where both Rainey's formulation and Newman's approximation provide the same results. Thus, 
Figure 28 - Amplitude of the nondimensional second-order moment, with respect to the mean water line, acting on the long (left $-\bar{\omega}_{j l}^{-}=0.827$ ) and short (right $\left.\bar{\omega}_{j l}^{-}=0.555\right)$ cylinders calculated with and without first-order motions as a function of the length of the shortest wave of the pair.
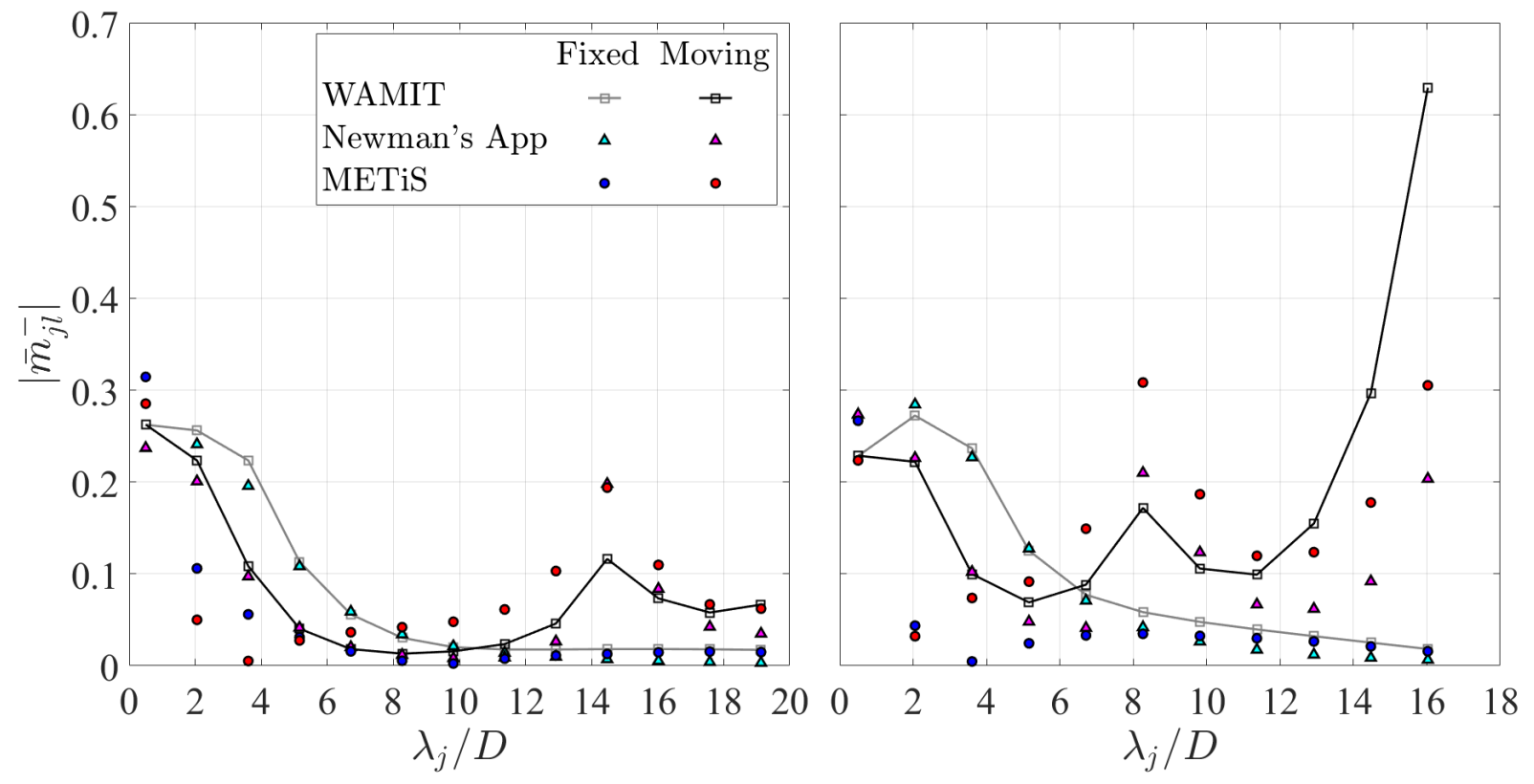

the complementarity between these approximations observed for the horizontal loads can not be exploited to analyze vertical forces. As pointed out by Molin and Chen (2002), this behavior suggests that while Newman's approximation models the part of the force that could be obtained with a far-field method, the slender-body approximation models local effects. 


\section{Analysis of the JPK floating wind turbine}

The JPK floating wind turbine was developed during a joint project between the University of São Paulo and the University of Tokyo that aimed at designing a FOWT for Brazilian waters (GONÇALVES et al., 2018). It consists of a semi-submersible hull with a $15 \mathrm{~m}$ diameter central column attached to three $9 \mathrm{~m}$ diameter columns arranged as an equilateral triangle, with the NREL 5MW reference turbine (JONKMAN; BUTTERFIELD, et al., 2009) mounted on top of a tower of height $69.5 \mathrm{~m}$, as illustrated in Figure 29. Among the objectives of the project was studying fundamental aspects of the dynamics of FOWTs, such as the impact of heave plates on floater motions (MELLO; MALTA, et al., 2021), the estimation of damping levels for slow-drift surge (CARMO; MELLO, et al., 2020) and the inclusion of rotor aerodynamics in wave tank tests (AMARAL et al., 2021).

Figure 29 - Illustration of the JPK floating wind turbine.

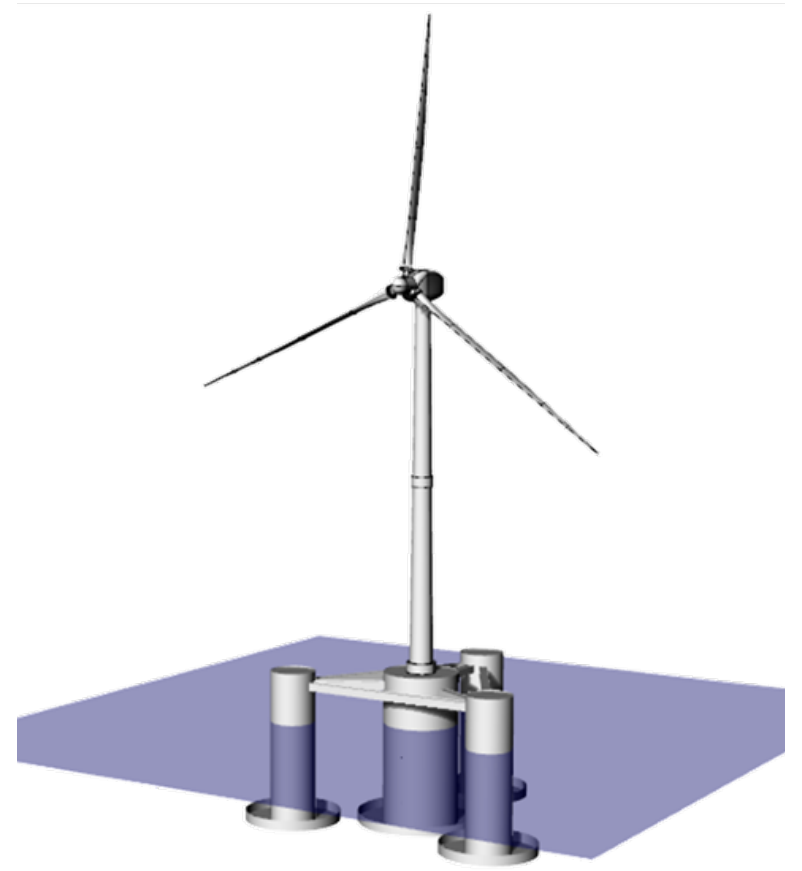

The project included two experimental campaigns conducted at the wave basin of the Numerical Offshore Tank of the University of São Paulo (TPN-USP) that comprised decay tests, regular, bichromatic and irregular waves. They both used the same hull model, built in a 1:80 scale, but they differ in that the first campaign considered waves only and a simple horizontal mooring system composed of springs, while the second one was moored using chain-polyethylene catenary lines and employed a wind actuator to model aerodynamic forces on the rotor using a software-in-the-loop approach. Moreover, the first phase included forced oscillations of the floater to evaluate hydrodynamic coefficients, which were not repeated in the second phase due to the hull being the same. The elastic 
properties of the prototype were not preserved in model scale in neither of the phases. Pictures of the models analyzed in each phase are given in Figure 30 (note the absence of heave plates, which is explained ahead).

Figure 30 - Pictures of the models analyzed in the first (left) and second (right) phases of the project.
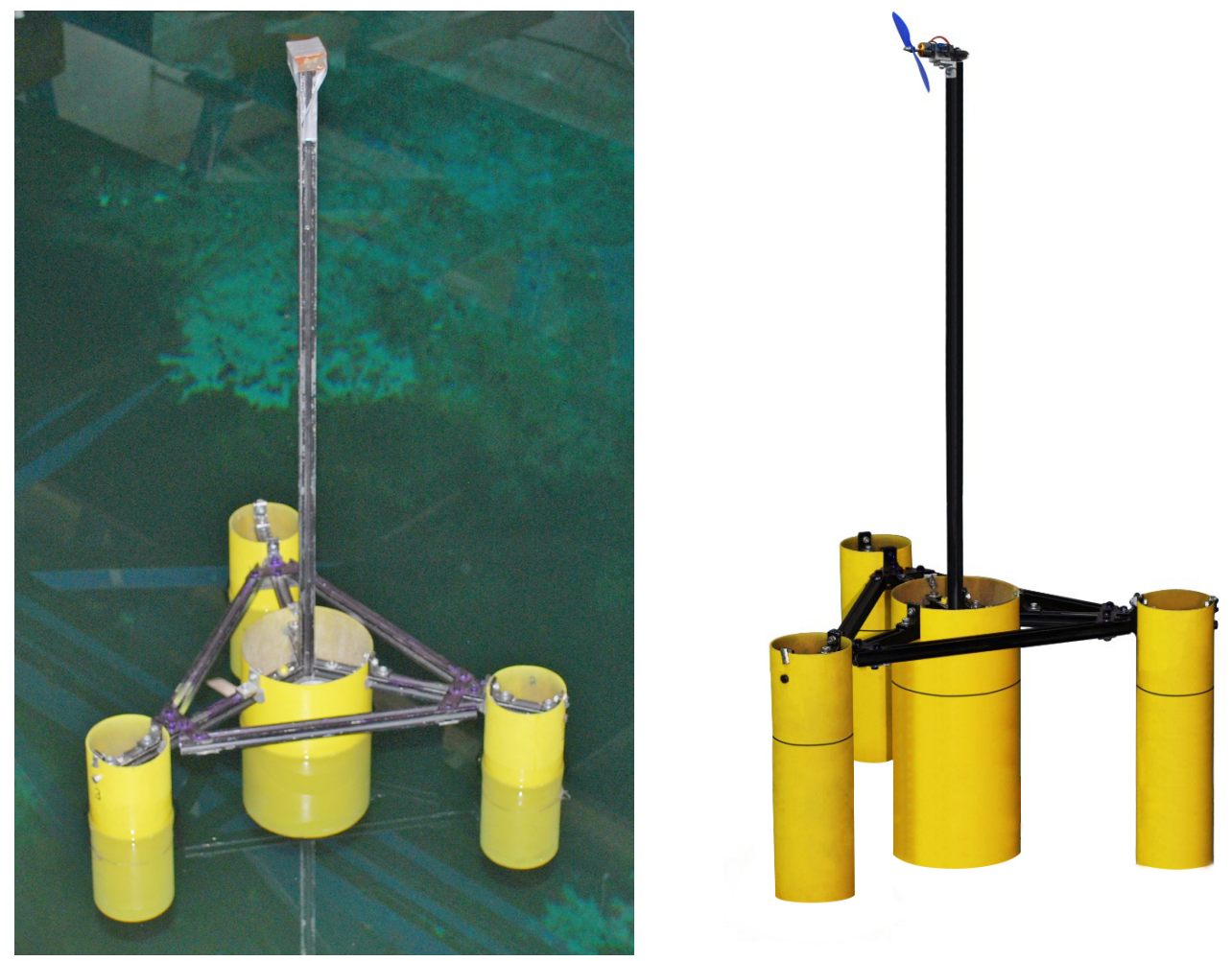

This thesis analyzes the results obtained in the second experimental campaign, comparing the motions of the floater under the action of waves calculated using the slender-body approximation with the ones measured in the experiments, as well as the ones computed with WAMIT and OpenFAST, a widely used open-source tool for the analysis of floating wind turbines that was formerly known as FAST (JONKMAN; BUHL, 2005). The results of the forced oscillations are used to assess the drag and added mass coefficients that are considered in the numerical models. Though heave plates are an important part of the design that has been extensively studied in the project, the presence of such structures is an unnecessary complication for the purposes of this thesis, as they render the flow more complex and make it harder to compare the slender-body approximation with radiation/diffraction theory. Besides, because the second-order module of WAMIT is incompatible with dipole panels, which are needed to properly represent thin elements such as heave plates, their inclusion would require workarounds that would further complicate the comparisons. Hence, only the version of the JPK FOWT without heave plates (which were built to be detachable) is analyzed here.

A software-in-the-loop scheme was used to emulate the aerodynamic forces on the rotor, but the results showed an unexpected change in the natural period of pitch that 
could not be explained yet. The main hypothesis is that the control loop is introducing a phase shift between the calculated force and the one that is actually applied by the rotor, but this still needs to be verified. Since explaining this phenomenon, which did not appear in the numerical simulations, is not part of the objectives of this thesis, the experimental results considering the concomitant action of waves and wind are, unfortunately, discarded. Thus, only numerical results are presented in the comparisons considering wind effects.

\subsection{Model properties and experimental setup}

The experimental campaign was conducted at the wave basin of TPN-USP, shown in Figure 31, which is a squared $14 \mathrm{~m} \times 14 \mathrm{~m} \times 4 \mathrm{~m}$ (length, width, depth) tank equipped with 152 active-absorption flap-type wave generators that can generate the incident waves with peak periods from $0.8 \mathrm{~s}$ to $2.5 \mathrm{~s}$ (equivalent to about $7.2 \mathrm{~s}$ and $22.4 \mathrm{~s}$ in full scale) and absorb part of the ones produced by the model (MELLO; CARNEIRO, et al., 2013).

Figure 31 - Wave basin of the Numerical Offshore Tank of the University of São Paulo.

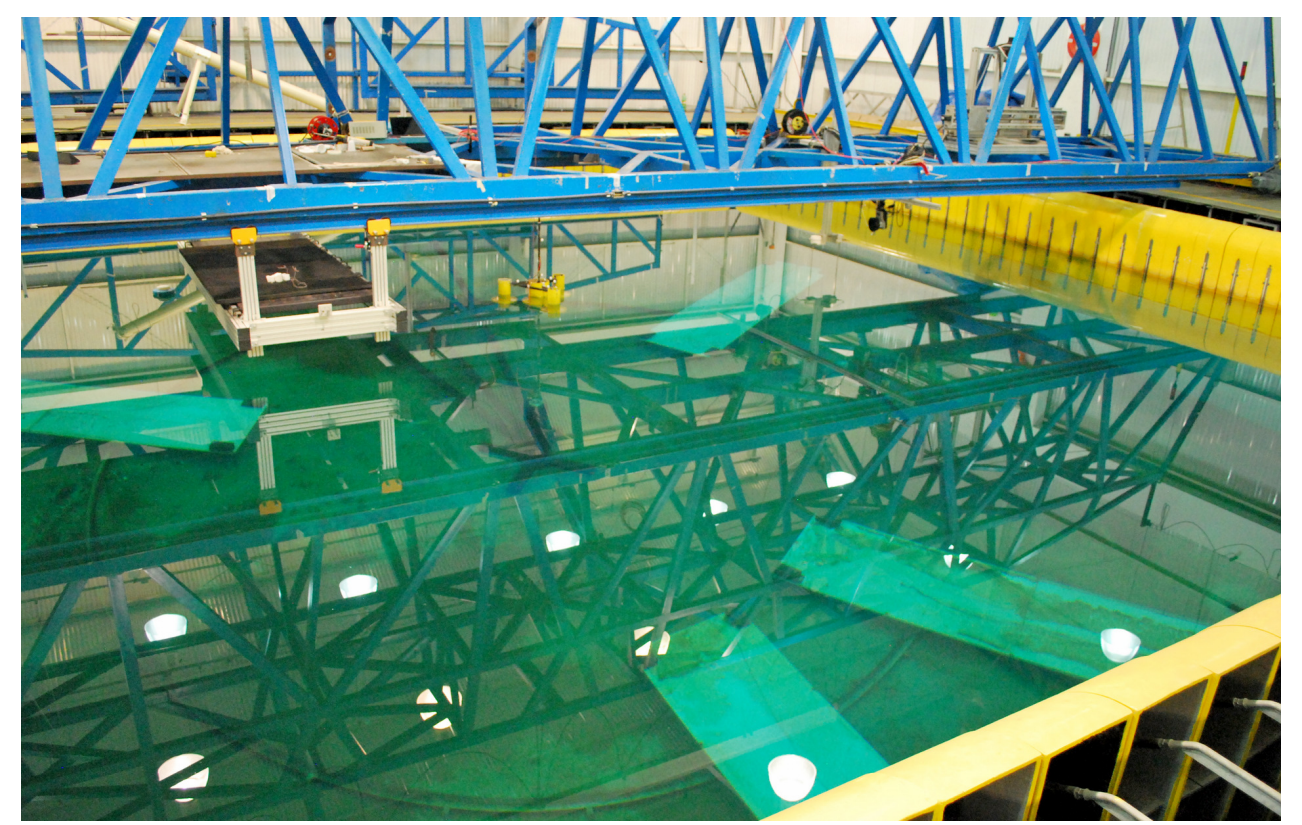

Source: Amaral et al. (2021)

The hull of the model was built in a 1:80 scale considering Froude scaling, and its main properties are given in Table 9 . The inertial properties were obtained by placing fixed ballast weights inside the columns, and their locations were established using Edtools ${ }^{\circledR}$, as illustrated in Figure 32. Due to removing the heave plates, the natural periods of heave and pitch are quite low.

Before placing the model in the tank, four wave probes were positioned to calibrate the waves, with one of them located at the origin of the coordinate system (the center of

\footnotetext{
${ }^{1}$ A software developed by Technomar Engenharia Oceânica https://www.technomar.com.br/
} 
Table 9 - Main properties of the JPK FOWT. Natural periods were obtained from decay tests of the moored model without wind emulation.

\begin{tabular}{lrr}
\hline & Full scale & Model Scale (1:80) \\
\hline Mass & $6936.0 \mathrm{t}$ & $13.55 \mathrm{~kg}$ \\
Displacement & $7351.3 \mathrm{~m}^{3}$ & $14.36 \mathrm{dm}^{3}$ \\
Diameter of central column & $15.0 \mathrm{~m}$ & $188 \mathrm{~mm}$ \\
Diameter of side columns & $9.0 \mathrm{~m}$ & $113 \mathrm{~mm}$ \\
Pitch/roll gyradius about center of mass & $21.9 \mathrm{~m}$ & $274 \mathrm{~mm}$ \\
Yaw gyradius about center of mass & $20.3 \mathrm{~m}$ & $254 \mathrm{~mm}$ \\
Draft & $20.0 \mathrm{~m}$ & $250 \mathrm{~mm}$ \\
KG & $15.6 \mathrm{~m}$ & $195 \mathrm{~mm}$ \\
KB & $10.0 \mathrm{~m}$ & $125 \mathrm{~mm}$ \\
BM & $8.9 \mathrm{~m}$ & $111 \mathrm{~mm}$ \\
GM & $3.3 \mathrm{~m}$ & $41 \mathrm{~mm}$ \\
\hline Surge/Sway natural period & $86.3 \mathrm{~s}$ & $9.65 \mathrm{~s}$ \\
Heave natural period & $9.8 \mathrm{~s}$ & $1.09 \mathrm{~s}$ \\
Pitch/Roll natural period & $21.0 \mathrm{~s}$ & $2.35 \mathrm{~s}$ \\
Yaw natural period & $47.0 \mathrm{~s}$ & $5.25 \mathrm{~s}$ \\
\hline
\end{tabular}

Figure 32 - Isometric and top views of the Edtools model showing the position of the ballast weights inside the columns.
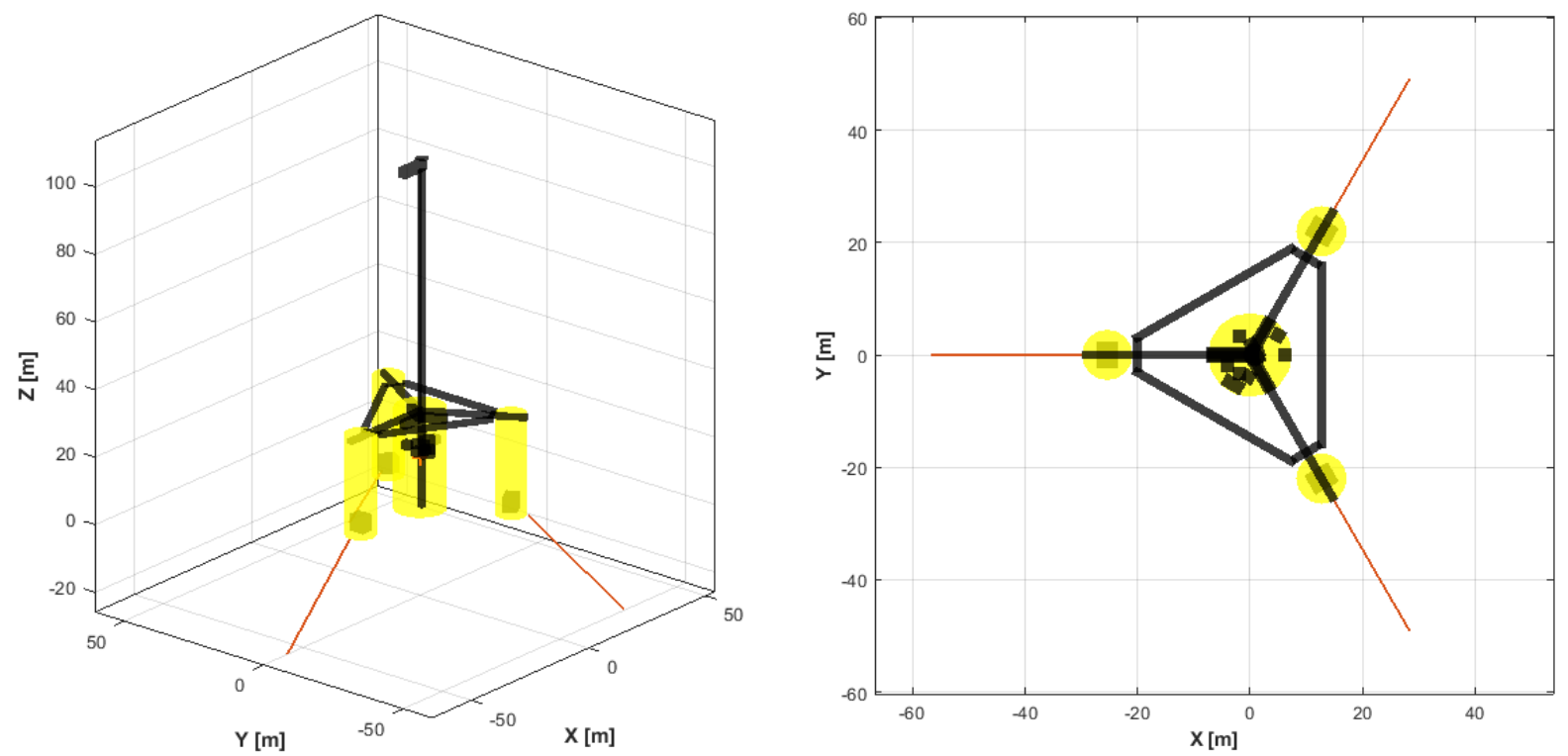

the tank). During the tests, the central wave probe was replaced by the model, but the other three were kept to measure the wave elevation and verify that they corresponded to the expected one. The motions of the model were measured using Qualisys ${ }^{\circledR}$ optical tracking system, consisting of four infrared cameras located on the instrumentation bridge that tracked the position of six markers attached to the model, with a sampling frequency of $100 \mathrm{~Hz}$ (which corresponds to a time discretization of $0.0894 \mathrm{~s}$ in full scale). 


\subsubsection{Rotor-nacelle-assembly (RNA)}

The JPK FOWT was designed considering the NREL 5MW reference turbine, which is described in details in Jonkman, Butterfield, et al. (2009). In the experiments, the RNA was represented by a wind actuator that modeled the aerodynamic thrust on the rotor using a software-in-the-loop approach, and even though the experimental results with wind are not discussed in this text, the ones obtained with numerical simulations are presented. As the inertial and elastic properties of the RNA were not preserved in the experiments (the total inertia of the FOWT is correct), only the geometric and aerodynamic properties are relevant. The main properties of the RNA are summarized in Table 10. Since only one wind speed was analyzed $\left(U_{w}=7.8 \mathrm{~m} / \mathrm{s}\right)$ and control of the rotor was not modeled, the rotor speed was kept constant and equal to $9.07 \mathrm{rpm}$ (it was turned off during the tests with waves only).

Table 10 - Main properties of the RNA.

\begin{tabular}{|c|c|}
\hline Rating & $5 \mathrm{MW}$ \\
\hline Number of blades & 3 \\
\hline Rotor diameter & $126 \mathrm{~m}$ \\
\hline Cut-in; rated; cut-out wind speed & $3 \mathrm{~m} / \mathrm{s} ; 11.4 \mathrm{~m} / \mathrm{s} ; 25 \mathrm{~m} / \mathrm{s}$ \\
\hline Cut-in; rated rotor & $6.9 \mathrm{rpm} ; 12.1 \mathrm{rpm}$ \\
\hline Rotor speed at $U_{w}=7.8 \mathrm{~m} / \mathrm{s}$ & $9.07 \mathrm{rpm}$ \\
\hline Hub height ${ }^{\dagger}$ & $81.48 \mathrm{~m}$ \\
\hline Hub diameter & $3 \mathrm{~m}$ \\
\hline Overhang & $5 \mathrm{~m}$ \\
\hline Shaft tilt ${ }^{\dagger}$ & $0^{\circ}$ \\
\hline Precone & $2.5^{\circ}$ \\
\hline Blade length & $61.5 \mathrm{~m}$ \\
\hline
\end{tabular}

${ }^{\dagger}$ The NREL 5MW reference turbine has a tilt angle of $5^{\circ}$ and a hub height of $90 \mathrm{~m}$, but the values above were considered in the tests.

\subsubsection{Mooring system}

The model was moored using three catenary mooring lines made of chain (bottom) and polyethylene (upper) segments, as illustrated in Figure 33. The fairleads were located at the center of the bottom of the side columns, thus $30 \mathrm{~m}$ distant from the axis of the central column, the anchor radius was $543 \mathrm{~m}$ and the water depth $302.8 \mathrm{~m}$. Table 11 summarizes the main characteristics of the mooring system.

\subsubsection{Environmental conditions}

Three sets of waves are considered in this work: a white-noise wave, which is used to obtain the first-order motion RAOs; ten bichromatic waves, for the analysis of lowfrequency surge motions (chosen in such a way that the difference-frequency between 
Figure 33 - Isometric and top views of the Edtools model illustrating the mooring lines. The chain segment is in blue, while the one made of polyethylene is in red.
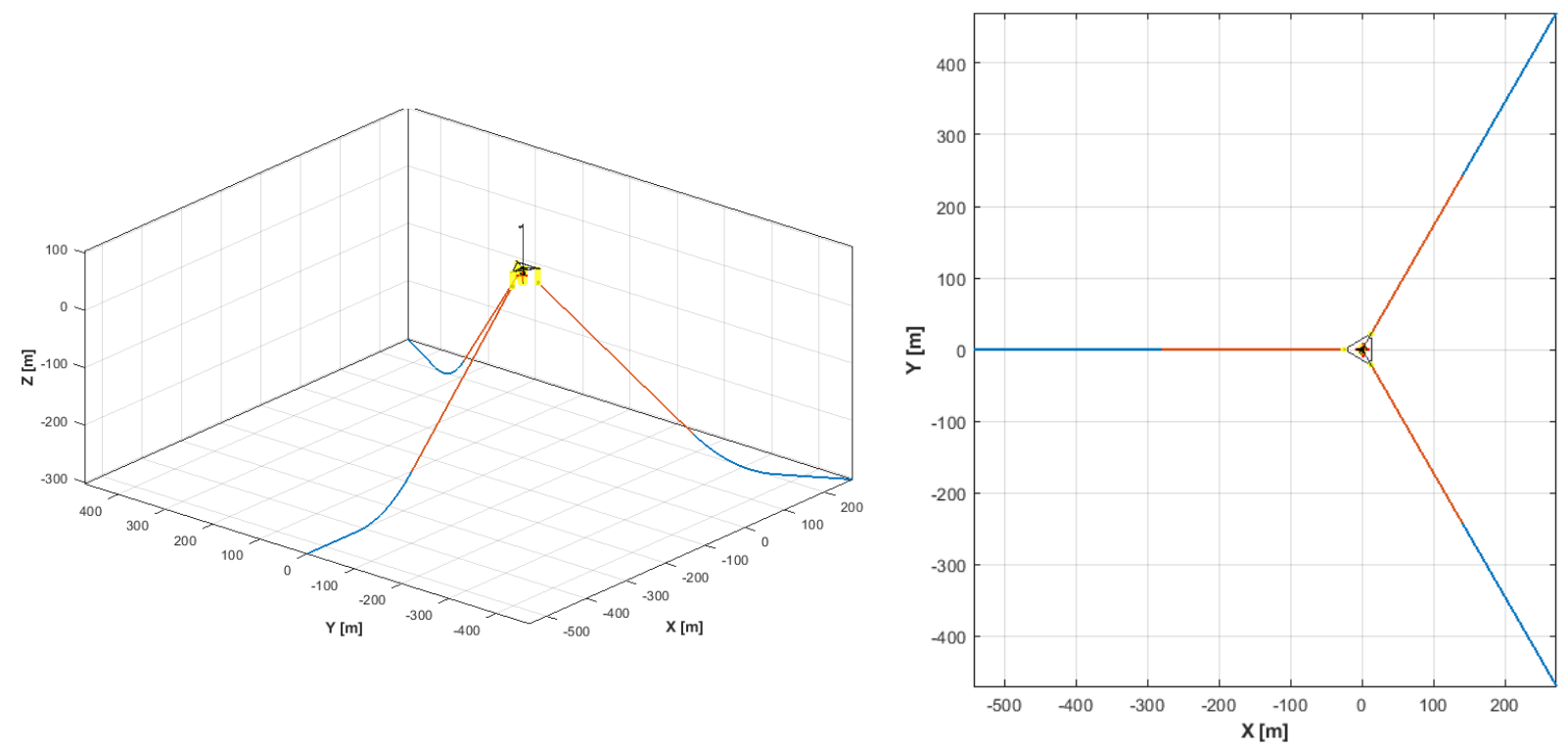

Table 11 - Main properties of the mooring system.

\begin{tabular}{lrr}
\hline & Full scale & Model scale (1:80) \\
\hline Anchors depth & $302.8 \mathrm{~m}$ & $3785 \mathrm{~mm}$ \\
Anchors radius from center & $534.1 \mathrm{~m}$ & $6789 \mathrm{~mm}$ \\
\hline Fairleads depth & $20 \mathrm{~m}$ & $250 \mathrm{~mm}$ \\
Fairleads radius & $30 \mathrm{~m}$ & $375 \mathrm{~mm}$ \\
\hline Bottom segment material & Chain & Chain \\
Length & $280 \mathrm{~m}$ & $3500 \mathrm{~mm}$ \\
Mass density & $1103.4 \mathrm{~kg} / \mathrm{m}$ & $0.172 \mathrm{~kg} / \mathrm{m}$ \\
Volume-equivalent diameter & $0.10 \mathrm{~m}$ & $0.0013 \mathrm{~m}$ \\
Adopted drag coefficient & 1.0 & 1.0 \\
\hline Upper segment material & Polyethylene & Polyethylene \\
Length & $347.6 \mathrm{~m}$ & $4345 \mathrm{~mm}$ \\
Mass density & $70.0 \mathrm{~kg} / \mathrm{m}$ & $0.011 \mathrm{~kg} / \mathrm{m}$ \\
Volume-equivalent diameter & $0.10 \mathrm{~m}$ & $0.0013 \mathrm{~m}$ \\
Adopted drag coefficient & 1.0 & 1.0 \\
\hline
\end{tabular}

${ }^{\dagger}$ More specifically, a multifilament polyethylene braided fishing line.

frequency pairs matches the natural frequency of surge observed in the decay tests, i.e. $\omega_{n 1}=0.073 \mathrm{rad} / s$, which corresponds to $T_{n 1}=86.3 \mathrm{~s}$ ); and four JONSWAP waves. Their characteristics are given in Tables 13, 14, and 15. Besides analyses considering waves only, the FOWT is also analyzed under the concomitant action of waves and wind (except for the bichromatic waves). Only one wind condition is considered, consisting of a constant speed of $U_{w}=7.8 \mathrm{~m} / \mathrm{s}$ (measured at a height of $81.48 \mathrm{~m}$ above the mean water surface) with an exponential profile with exponent 0.2 , as summarized in Table 12. 
In this text, most of the analyses consider an incidence of $180^{\circ}$ (i.e. coming from $+\mathrm{x}$ to $-\mathrm{x}$ ) for both the waves and the wind, except for a brief set of results concerning the first-order forces for a wave incidence of $210^{\circ}$ (a counterclockwise rotation of $30^{\circ}$ with respect to $180^{\circ}$ ) that are presented in Section 6.5.1.

Table 12 - Wind condition considered in the tests.

\begin{tabular}{lr}
\hline Wind speed & $7.8 \mathrm{~m} / \mathrm{s}$ \\
Ref. Height & $81.48 \mathrm{~m}$ \\
Exponent & 0.2 \\
Air density & $1.225 \mathrm{~kg} / \mathrm{m}^{3}$ \\
\hline
\end{tabular}

Table 13 - White-noise wave characteristics.

\begin{tabular}{ccccccccc}
\hline \multirow{2}{*}{ ID } & \multicolumn{4}{c}{ Full scale } & \multicolumn{4}{c}{ Model scale } \\
\cline { 2 - 9 } & $T_{\min }(\mathrm{s})$ & $T_{\max }(\mathrm{s})$ & $H_{s}(\mathrm{~m})$ & Duration $(\mathrm{s})$ & $T_{\min }(\mathrm{s})$ & $T_{\max }(\mathrm{s})$ & $H_{s}(\mathrm{~mm})$ & Duration $(\mathrm{s})$ \\
\hline WHI01 & 6.5 & 25.0 & 0.50 & 1270 & 0.73 & 2.80 & 6.25 & 142 \\
\hline
\end{tabular}

Table 14 - Characteristics of the bichromatic waves $\left(\omega_{1}-\omega_{2}=0.073 \mathrm{rad} / \mathrm{s}\right)$. Each component has a height equal to $1 \mathrm{~m}$.

\begin{tabular}{ccccc}
\hline \multirow{2}{*}{ ID } & \multicolumn{2}{c}{ Full scale } & \multicolumn{2}{c}{ Model scale } \\
\cline { 2 - 5 } & $T_{1}(\mathrm{~s})$ & $T_{2}(\mathrm{~s})$ & $T_{1}(\mathrm{~s})$ & $T_{2}(\mathrm{~s})$ \\
\hline BIC01 & 8.97 & 10.00 & 1.00 & 1.12 \\
$\mathrm{BIC02}$ & 9.76 & 11.00 & 1.09 & 1.23 \\
$\mathrm{BIC03}$ & 10.00 & 11.30 & 1.12 & 1.26 \\
$\mathrm{BIC04}$ & 10.54 & 12.00 & 1.18 & 1.34 \\
$\mathrm{BIC05}$ & 11.00 & 12.60 & 1.23 & 1.41 \\
$\mathrm{BIC06}$ & 11.30 & 13.00 & 1.26 & 1.45 \\
$\mathrm{BIC07}$ & 12.00 & 13.93 & 1.34 & 1.56 \\
$\mathrm{BIC08}$ & 12.05 & 14.00 & 1.35 & 1.57 \\
$\mathrm{BIC09}$ & 13.00 & 15.29 & 1.45 & 1.71 \\
$\mathrm{BIC10}$ & 14.00 & 16.70 & 1.57 & 1.87 \\
\hline
\end{tabular}

Table 15 - Characteristics of the JONSWAP waves analyzed. They were tested for a duration of $3 \mathrm{~h}$ in full scale (1207 $\mathrm{s}$ in model scale).

\begin{tabular}{ccccccc}
\hline \multirow{2}{*}{ ID } & \multicolumn{2}{c}{ Full scale } & \multicolumn{2}{c}{ Model scale } & \multicolumn{2}{c}{ Nondimensional } \\
\cline { 2 - 7 } & $T_{p}(\mathrm{~s})$ & $H_{s}(\mathrm{~m})$ & $T_{p}(\mathrm{~s})$ & $H_{s}(\mathrm{~mm})$ & $\gamma$ & Aver. Steepness \\
\hline JON01 & 8.0 & 1.0 & 0.894 & 13 & 2.31 & $1.00 \%$ \\
JON02 & 12.0 & 2.0 & 1.342 & 25 & 1.89 & $0.89 \%$ \\
JON03 & 18.0 & 4.0 & 2.012 & 50 & 1.55 & $0.79 \%$ \\
JON04 & 18.0 & 8.0 & 2.012 & 100 & 1.55 & $1.58 \%$ \\
\hline
\end{tabular}




\subsection{Forced oscillations}

A set of forced oscillation tests of the whole floater in different conditions was performed to evaluate the added mass and drag coefficients to feed the numerical models. For doing so, a linear actuator was assembled at the center of the tank, as shown in Figure 34. The model was attached to the actuator and forced to move either horizontally (surge and sway) or vertically (heave) following a prescribed sinusoidal motion with six different amplitudes and two distinct periods, as listed in Tables 16 and 17. The period of $12 \mathrm{~s}$ was chosen to represent a typical value of ocean waves, while $84.4 \mathrm{~s}$ is sufficiently close to the natural period of surge (it is the natural period of a version of the model equipped with heave plates, which was the focus of the experimental campaign). Since the largest period is of interest only for the surge motion, it was included in the horizontal oscillation tests only. The force exerted by the actuator was measured using four load cells with a capacity of $10 \mathrm{kgf}$ each assembled between two parallel plates, with a sampling rate of $100 \mathrm{~Hz}$.

The amplitude of motion and the period of oscillation can be made nondimensional as follows:

$$
\begin{gathered}
K C=\frac{2 \pi A}{D} \\
\beta=\frac{R e}{K C}=\frac{D^{2}}{T \nu}
\end{gathered}
$$

with $A$ the motion amplitude, $D$ a characteristic length, $\nu=1.009 \mathrm{E}-6 \mathrm{~m}^{2} / \mathrm{s}$ the kinematic viscosity of the water, $T$ the period of motion and $R e$ the Reynolds number. The resulting quantities $K C$ and $\beta$ are, respectively, the Keulegan-Carpenter number and the frequency parameter (SARPKAYA, 1976), which are known to be the main nondimensional numbers that characterize the hydrodynamic coefficients of circular cylinders in sinusoidal flow (SARPKAYA, 2010). While $K C$ is simply a length ratio that measures the relative importance between drag and inertia forces, being related to the relevance of separation effects, the frequency parameter accounts for dynamic aspects of the flow. Due to the adoption of Froude's scaling, the values of $\beta$ from the experiment are not representative of the full scale case.

Both the nondimensional parameters above were originally introduced for the analysis of cylinders, but only forced oscillations of the whole floater were performed in this work. While for the cylinder the choice of characteristic length is evident, this is not the case here, and there could be some debate regarding the correct length to be chosen in this case. For the horizontal motion of the cylinder, one reasonable choice of characteristic length is the mean diameter of the columns:

$$
D_{x}=\frac{1}{N} \sum_{i=1}^{N} D_{i}=10.5 \mathrm{~m}
$$


Figure 34 - Pictures of the setup of the horizontal (left) and vertical (right) forced oscillation tests.
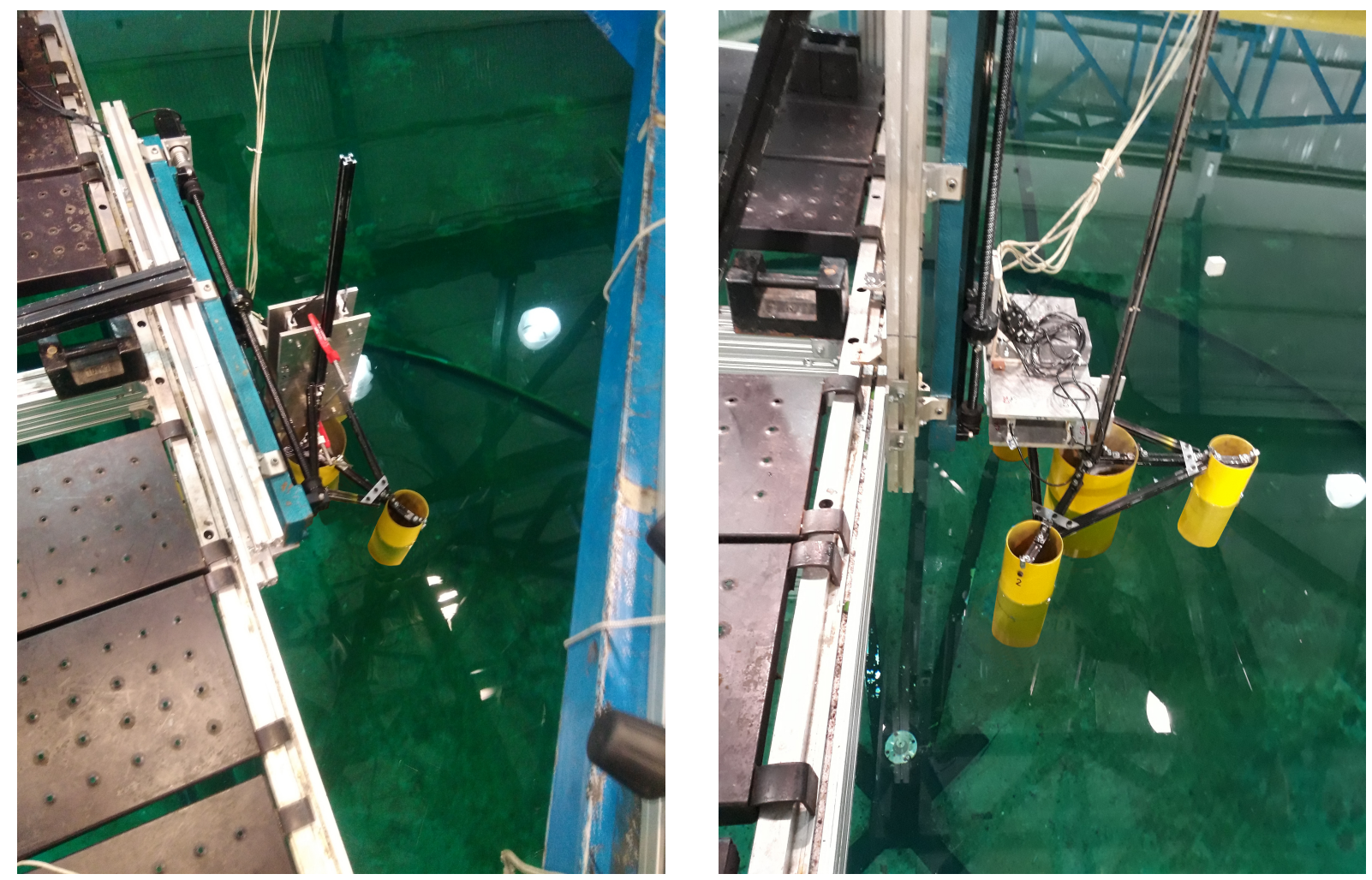

Table 16 - Motion amplitudes of the forced oscillations and corresponding $K C$ values.

\begin{tabular}{|c|c|c|c|}
\hline \multicolumn{2}{|c|}{ Amplitude (m) } & \multirow{2}{*}{$K C_{x}$} & \multirow{2}{*}{$K C_{z}$} \\
\hline Full scale & Model scale & & \\
\hline 0.800 & 0.010 & 0.48 & 0.23 \\
\hline 1.600 & 0.020 & 0.96 & 0.46 \\
\hline 2.400 & 0.030 & 1.44 & 0.69 \\
\hline 3.200 & 0.040 & 1.91 & 0.93 \\
\hline 4.000 & 0.050 & 2.39 & 1.16 \\
\hline 4.800 & 0.060 & 2.87 & 1.39 \\
\hline
\end{tabular}

Table 17 - Motion periods considered in the forced oscillations and corresponding $\beta$ values.

\begin{tabular}{cccccc}
\hline & Full scale & \multicolumn{3}{c}{ Model scale } \\
Period (s) & $\beta_{x}$ & $\beta_{z}$ & Period $(\mathrm{s})$ & $\beta_{x}$ & $\beta_{z}$ \\
\hline 12.0 & $9.11 \mathrm{E}+06$ & $3.85 \mathrm{E}+07$ & 1.34 & $1.27 \mathrm{E}+04$ & $5.39 \mathrm{E}+04$ \\
84.4 & $1.29 \mathrm{E}+06$ & - & 9.43 & $1.81 \mathrm{E}+03$ & - \\
\hline
\end{tabular}

with $D_{i}$ the diameter of each column. For the vertical motions, the diameter of the circle whose surface is equal to the projected area of the hull (in the $z$ direction) is adopted:

$$
D_{z}=\sqrt{\sum_{i=1}^{N} D_{i}^{2}}=21.6 \mathrm{~m}
$$


Besides the definition of the characteristic length, it is also necessary to choose a characteristic volume and area to define the added mass and drag coefficients in the horizontal and vertical directions. Following the same convention established in Chapter 3, the volumes and areas below are considered:

$$
\begin{aligned}
& \mathcal{V}_{x}=d \frac{\pi}{4} \sum_{i=1}^{N} D_{i}^{2}=7351.3 \mathrm{~m}^{3} \\
& \mathcal{V}_{z}=\frac{4 \pi}{3} \sum_{i=1}^{N}\left(\frac{D_{i}}{2}\right)^{3}=2912.3 \mathrm{~m}^{3} \\
& \mathcal{S}_{x}=d \sum_{i=1}^{N} D_{i}=840.0 \mathrm{~m}^{2} \\
& \mathcal{S}_{z}=\frac{\pi}{4} \sum_{i=1}^{N} D_{i}^{2}=367.6 \mathrm{~m}^{2}
\end{aligned}
$$

with $d$ being the draft.

\subsubsection{Results}

The coefficients obtained from the forced oscillations with the procedure detailed in Appendix E are plotted in Figure 35, which also includes the added mass coefficients obtained with WAMIT at the corresponding period of motion.

Concerning $C_{a x}$, the experimental values are within $7 \%$ of the solution considering potential theory, which is quite a good agreement. The experimental values of $C_{a z}$, however, are considerably different from the one obtained with WAMIT, with differences ranging from $17 \%$ to $43 \%$ with respect to the potential value $C_{a z}=0.50$. This can either indicate that viscous effects significantly affect the vertical added mass or that this larger relative difference is simply due to the measured vertical force being smaller.

The $C_{D x}$ values of both frequencies are quite similar around $1.5<K C<3$, but the values diverge as $K C$ is reduced. The general trend is very different from the one observed by Sarpkaya (1986) for circular cylinders at low $K C$ numbers, which would be steady reduction of $C_{D}$ as $K C$ increases up to a certain point, but this difference may be attributed to the aspect ratios of the columns of the JPK floater $(L / D=1.33$ for the main column and 2.22 for the others) being far lower than that of the cylinders analyzed in his work $(L / D=24,12$ and 6.85$)$ and to interactions between the columns.

How to extend these results to the analysis of a structure under the action of waves, however, is not evident. First, as only oscillations of the whole floater were performed, it is difficult to establish the coefficients of each column of the hull. But, most importantly, one may wonder how to account for the large array of wave frequencies that are present in 
Figure 35 - Added mass and drag coefficients obtained from the forced oscillations.

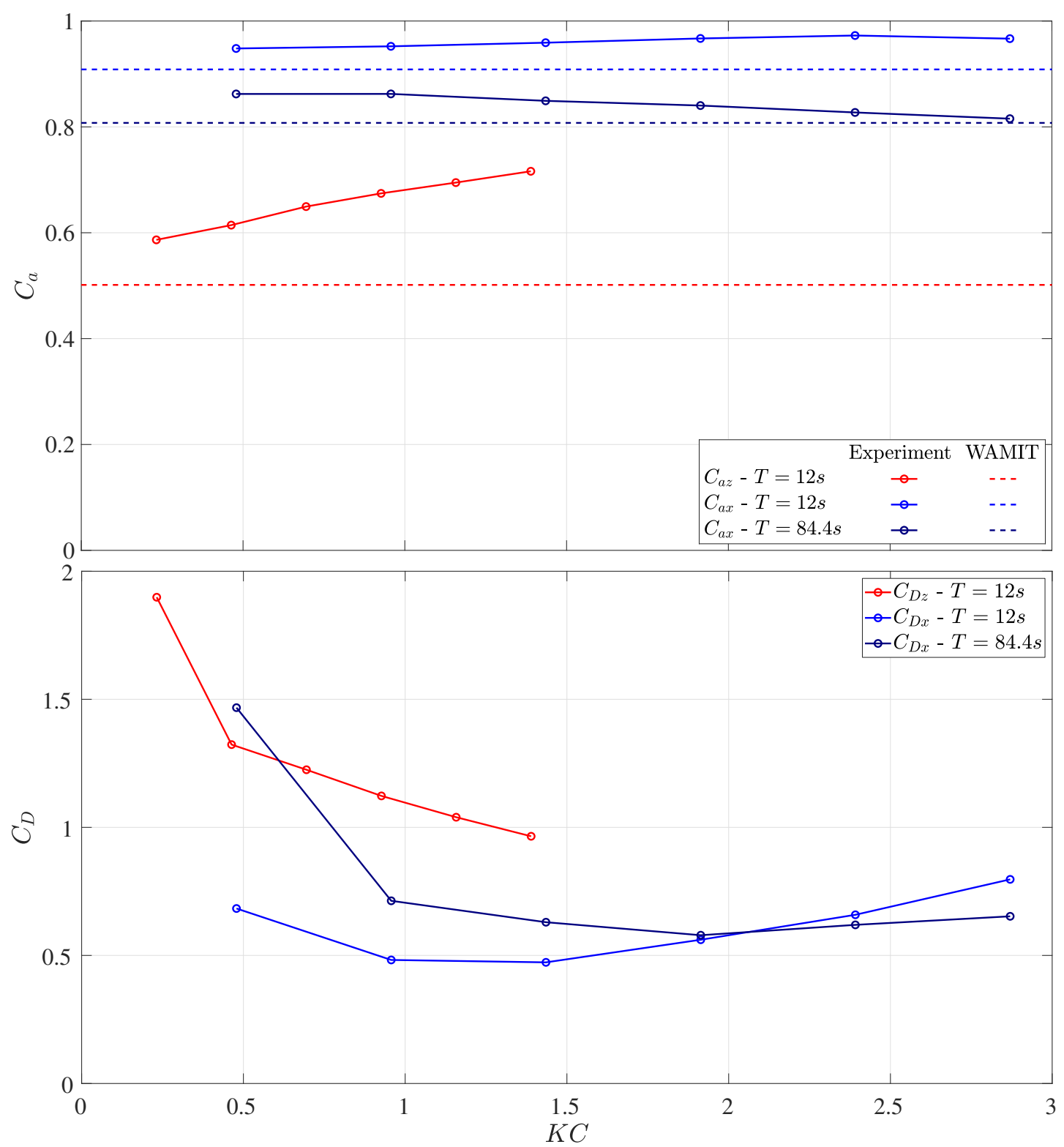

a real sea and, if slow-drift is of interest, how to take into account the fact that the body is simultaneously oscillating in very different frequencies (wave and slow-drift). Regarding the added mass coefficients, a simple approach is to consider it to be the same for all the columns. And though they have an impact on the forces calculated with the slender-body approximation, it is more important to ensure that the resulting natural periods are correct, which in this case is achieved within reasonable limits by considering $C_{a x}=0.82$ and $C_{a z}=0.68$ (calculated neglecting coupling effects). These values are within the range observed in the forced oscillations, and are kept the same for all the wave conditions analyzed in this work.

The choice of the drag coefficients, however, is not as simple. In a previous work concerning the same model (CARMO; MELLO, et al., 2020), good results were obtained for the slow surge of the JPK FOWT under the action of bichromatic waves by using 
$C_{D x}$ obtained from the low-frequency forced oscillations, considered to be the same for all columns. The procedure is based on calculating the $K C$ number using the amplitudes of slow surge motion measured in the experiments, $A_{x}^{-}$, i.e.:

$$
K C_{x}=\frac{2 \pi A_{x}^{-}}{D_{x}}
$$

which corresponds to a $C_{D x}$ value from the results given in Figure 35. As the numerical analysis in that work used WAMIT, which is a frequency domain code, an equivalent linear damping coefficient, $B_{11}^{\text {ext }}$, was calculated by imposing that it must dissipate the same energy as the quadratic drag force at each motion cycle, resulting in the following relation for a sinusoidal motion:

$$
B_{11}^{\mathrm{ext}}=\frac{8}{3 \pi} \omega_{n 1} A_{x}^{-} B_{\mathrm{quad}}
$$

with $B_{\text {quad }}=\frac{1}{2} \rho C_{D x} \mathcal{S}_{x}$ and $\omega_{n 1}$ the frequency of motion, which in this case is the difference frequency of the bichromatic waves, chosen to be equal to the natural frequency of surge motion. This $B_{11}^{\text {ext }}$ can be expressed in terms of the damping level, $\zeta_{1}$, defined as:

$$
\zeta_{1}=\frac{B_{11}^{\text {ext }}}{B_{1}^{\text {crit }}}
$$

where $B_{1}^{\text {crit }}=1.90 \mathrm{E}+06 \mathrm{~N} . \mathrm{s} / \mathrm{m}$ is the critical damping in surge (considering $C_{a x}=0.82$ ).

This course of action was well suited to that work because the objective was to verify the possibility of reproducing the experiments with waves using the results from simplified model tests such as forced oscillations and decay tests. Since the tests with bichromatic waves presented in this thesis have a similar objective, i.e. to assess whether the force models are capable of reproducing the slow surge motion of the floater observed in the experiments, that approach is also followed in this case. Besides, it is interesting to make the other aspects of the problem, specially the first-order motions, as close as possible to the experimental ones. For this reason, the same approach is followed to compute $C_{D z}$, but this time based on the heave amplitude, given by $A_{z}^{(1)}=\sqrt{2} \operatorname{std}\left(\xi_{3}\right)$, where "std" denotes the standard deviation. The resulting coefficients, including $B_{11}^{\text {ext }}$ for the WAMIT simulations, are given in Table 18. Note that some $K C$ numbers are outside the range analyzed in the experiments, requiring an extrapolation of the experimental data to obtain the corresponding drag coefficients. For doing so, a cubic spline extrapolation was chosen.

On the other hand, the simulations considering a white-noise and JONSWAP waves aim at testing METiS as a design tool, verifying its capability to reproduce the first and second-order motions of the FOWT with and without wind. In this case, it would be troublesome if the software required previous knowledge of the motion amplitudes to be used, so a more predictive approach is employed to analyze these wave conditions. For the cases considering a white-noise wave, only the first-order motions of the FOWT with and without wind are of interest, and since heave, roll and pitch are the only degrees of freedom 
Table 18 - Motion amplitudes calculated from the experiments considering bichromatic waves (incidence equal to $180^{\circ}$ ) without wind, with their respective KeuleganCarpenter numbers and resulting $C_{a}, C_{D}$ and $\zeta_{1}$ obtained from forced oscillations.

\begin{tabular}{c|cccc|ccc}
\hline Wave ID & $A_{x}^{-}(m)$ & $K C_{x}$ & $C_{D, x}$ & $\zeta_{1}$ & $A_{z}^{(1)}(m)$ & $K C_{z}$ & $C_{D, z}$ \\
\hline BIC01 & 1.7 & 1.02 & 0.68 & $1.6 \%$ & 1.4 & 0.40 & 1.42 \\
BIC02 & 1.8 & 1.09 & 0.66 & $1.6 \%$ & 1.6 & 0.47 & 1.32 \\
BIC03 & 1.6 & 0.94 & 0.72 & $1.6 \%$ & 1.4 & 0.42 & 1.38 \\
BIC04 & 1.0 & 0.60 & 1.18 & $1.6 \%$ & 1.2 & 0.35 & 1.50 \\
BIC05 & 1.0 & 0.59 & 1.20 & $1.6 \%$ & 1.1 & 0.32 & 1.58 \\
BIC06 & 0.8 & 0.48 & 1.45 & $1.6 \%$ & 0.9 & 0.27 & 1.73 \\
BIC07 & 0.6 & 0.33 & 1.95 & $1.6 \%$ & 0.9 & 0.26 & 1.80 \\
BIC08 & 0.6 & 0.34 & 1.92 & $1.6 \%$ & 0.9 & 0.26 & 1.79 \\
BIC09 & 0.4 & 0.24 & 2.35 & $1.3 \%$ & 0.7 & 0.20 & 2.04 \\
BIC10 & 0.4 & 0.22 & 2.45 & $1.3 \%$ & 0.7 & 0.21 & 2.00 \\
\hline
\end{tabular}

that have natural periods within the range of the incoming waves, only $C_{D z}$ is actually relevant (the impact of $C_{D x}$ on roll/pitch is far smaller than that of $C_{D z}$ ). Besides, due to the very low significant height of the sea $\left(H_{S}=0.50 \mathrm{~m}\right)$, small motions are expected, hence the coefficients should be chosen for low $\mathrm{KC}$ values. Considering the values at the lowest $K C$ that were tested, a value of $C_{D z}=1.50$ seems to be a good estimation, while $C_{D x}$ is taken as 1.0.

Though different drag coefficients are expected for different levels of motion, the coefficients adopted for the WHI01 wave are taken as a first estimation for the floater in general conditions, including the JONSWAP waves, as listed in Table 19. These drag coefficients, however, should be seen only as a reasonable estimation of the drag force acting on the structure, and, when suitable, simulations considering other values are also presented to assess their impact on the results. In these cases, the values adopted will be indicated in the text.

Table 19 - Drag and added mass coefficients of the columns of the JPK considered in general conditions, including the WHI and JON waves.

\begin{tabular}{cccc}
\hline$C_{a x}$ & $C_{a z}$ & $C_{D x}$ & $C_{D z}$ \\
\hline 0.82 & 0.68 & 1.00 & 1.50 \\
\hline
\end{tabular}




\subsection{Numerical models}

Numerical analyzes of the JPK FOWT were performed using METiS, WAMIT and OpenFAST. The objective is to compare the results obtained with the numerical tool developed in this thesis not only with the experiments, but also with the responses calculated by two state-of-the-art codes that are widely used in the analysis of FOWTs. As each of these tools follows a different modeling approach, these comparisons evidence the capabilities and limitations of each method.

\subsubsection{WAMIT}

Like in Chapter 5, WAMIT simulations were performed with version $6.106 \mathrm{~S}$ of the software. The submerged part of the hull was represented by a higher-order mesh and, due to the symmetry of the problem, only one side of the body was meshed, as illustrated in Figure 36. The interior free surface was also discretized in order to remove irregular frequencies. There are some differences between the case without and with wind, namely an additional damping and a steady tilt of the hull model in the latter case, and the impact of the wind is discussed at the end of this section.

Figure 36 - Higher-order mesh of the JPK floater.

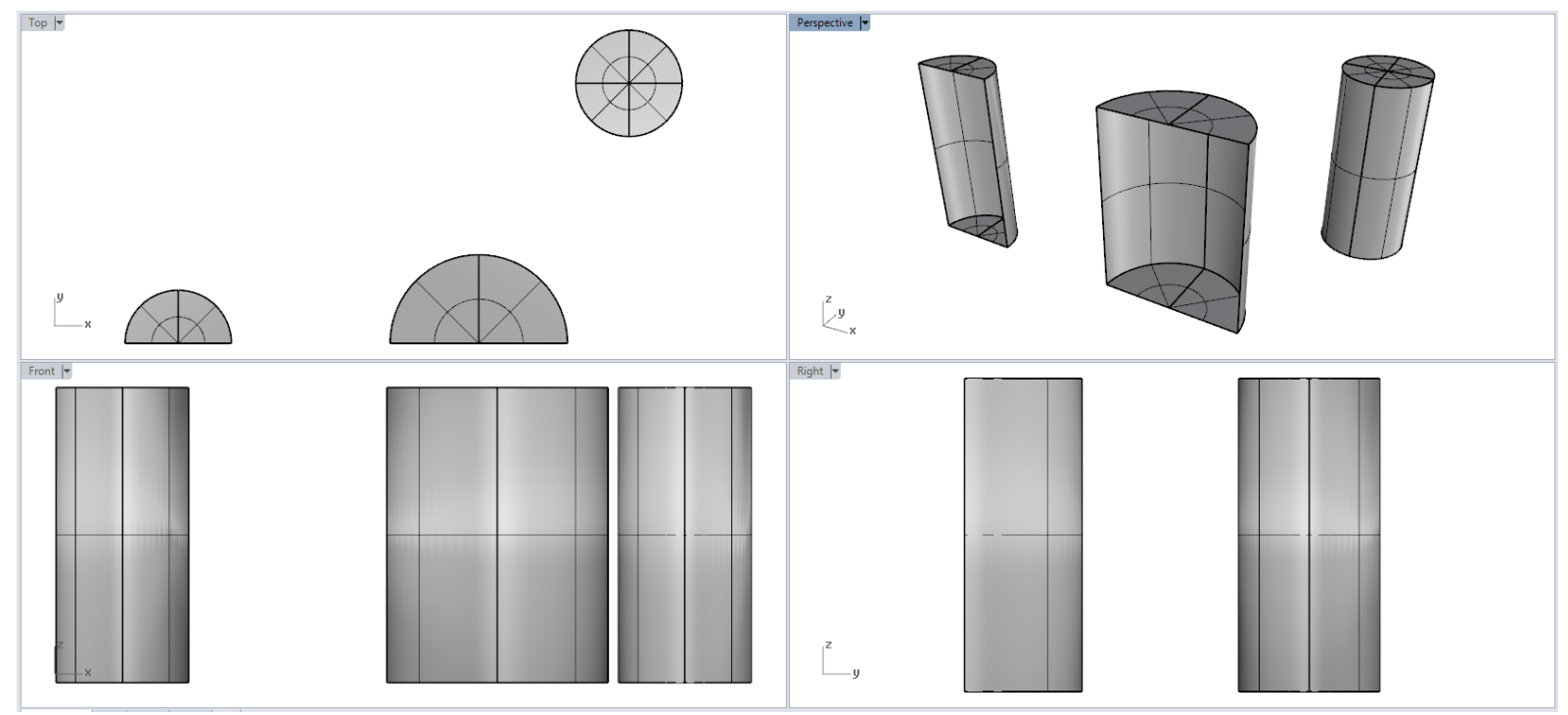

Similar to what was done in the case of the single cylinder, the panel size was set via the panel size parameter. Its value was chosen based on a convergence analysis of the mean surge force with three different meshes: a coarse one, with panel size 5.0; a medium mesh, with panel size 2.5; and a fine one with panel size 1.25. The mean surge force was chosen to assess the numerical convergence because it is a second-order quantity that depends only on the first-order solution, thus allowing a relatively fast evaluation of mesh convergence. The results, illustrated in Figure 37, show that some variation between the fine, medium and coarse mesh can be observed below $2 \mathrm{~s}$, but since there is no wave energy 
Figure 37 - Mean surge force on the floater for different panel sizes.

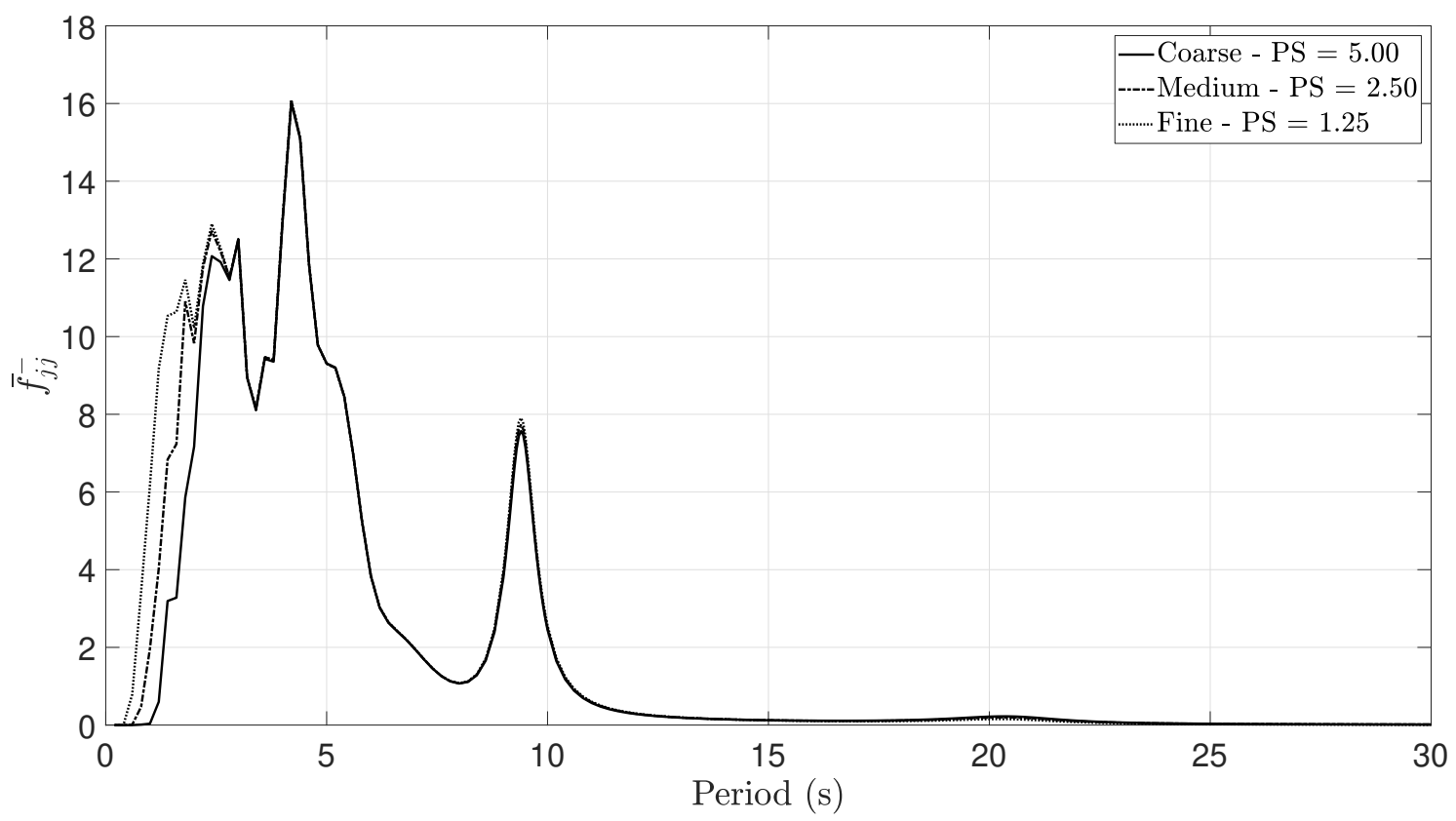

below $4 \mathrm{~s}$ for any of the waves analyzed, a panel size of 5.0 would be enough. Nevertheless, the panel size equal to 2.5 was chosen for a larger safety margin.

If WAMIT results were used only to provide first-order frequency-dependent hydrodynamic inputs for OpenFAST simulations (namely added mass, potential damping and first-order diffraction loads), the specifications above would be enough. However, as the simulations include second-order loads, it is important to properly model the first-order motions of the body in the WAMIT analysis, meaning that mooring and viscous effects need to be accounted for. The action of the mooring system was modeled by the following external linear stiffness matrix, which was evaluated using the analytical formulation proposed by Pesce, Amaral, and Franzini (2018) and Amaral (2020) around the mean body position (taken as the initial body position, since mean drift was small for the cases without wind):

$$
\mathbb{K}_{M, G}=\left[\begin{array}{cccccc}
6.92 \mathrm{e} 4 & 0 & 0 & 0 & 5.37 \mathrm{e} 5 & 0 \\
0 & 6.92 \mathrm{e} 4 & 0 & -5.37 \mathrm{e} 5 & 0 & 0 \\
0 & 0 & 1.04 \mathrm{e} 5 & 0 & 0 & 0 \\
0 & -5.37 \mathrm{e} 5 & 0 & 1.07 \mathrm{e} 8 & 0 & 0 \\
5.37 \mathrm{e} 5 & 0 & 0 & 0 & 1.07 \mathrm{e} 8 & 0 \\
0 & 0 & 0 & 0 & 0 & 9.12 \mathrm{e} 7
\end{array}\right]
$$

with $G$ indicating that the moments are taken with respect to the center of gravity. The units are $\mathrm{kg}, \mathrm{m}$ and $\mathrm{s}$.

Since WAMIT is a frequency-domain software, the only way to consider viscous effects is via an external linear damping matrix. However, as viscous loads are modeled in the other software by the quadratic drag from Morison's equation, the damping levels 
corresponding to a given combination of drag coefficients is dependent on the amplitudes of motions. This means that a different damping matrix should be considered for each sea condition, but this is a very unpractical approach. Instead, for the sake of simplicity, WAMIT simulations were performed considering one fixed external linear damping matrix with terms $B_{33}^{\text {ext }}$ and $B_{55}^{\text {ext }}$ set to approximately match the first-order heave and pitch RAOs calculated with the white-noise wave in OpenFAST, as presented later in Section 6.5.2. The resulting values are given in Table 20 .

Table 20 - External linear heave and pitch damping considered in the WAMIT simulations.

\begin{tabular}{lccc}
\hline & External damping & Critical damping & Damping level \\
\hline Heave $($ N.s $/ \mathrm{m})$ & $2.90 \mathrm{E}+05$ & $1.15 \mathrm{E}+07$ & $2.5 \%$ \\
Pitch $($ N.m.s $/ \mathrm{rad})$ & $1.70 \mathrm{E}+08$ & $2.37 \mathrm{E}+09$ & $7.2 \%$ \\
\hline
\end{tabular}

The second-order results needed from WAMIT for the OpenFAST simulations are the Quadratic Transfer Functions (QTFs) of surge, heave and pitch loads. They were computed for frequencies ranging from $0.15 \mathrm{rad} / \mathrm{s}$ to $1.80 \mathrm{rad} / \mathrm{s}$ (roughly from $3.4 \mathrm{~s}$ to $42 \mathrm{~s}$ ) with a frequency resolution of $0.25 \mathrm{rad} / \mathrm{s}$. This range was chosen in such a way that the QTF diagonals corresponding to a difference frequency close to the surge and pitch natural frequencies $(0.073 \mathrm{rad} / \mathrm{s}$ and $0.299 \mathrm{rad} / \mathrm{s}$, respectively) would encompass all the frequencies with wave energy of the JONSWAP seas described in Section 6.1.3. As no significant slow heave was observed, it was not taken into account to determine the frequency range.

A very useful approximation that significantly simplifies and speeds up the analysis is to neglect the free-surface forcing terms in the solution of the second-order potential, allowing the problem to be solved without meshing the free surface. Since this approximation is adequate for the case of a single cylinder, it is reasonable to expect that it is also valid for the case of a structure composed of several cylinders. Nevertheless, in order to verify whether this approximation is indeed applicable, the frequency pairs that are the closest to a difference frequency equal to the natural frequencies of surge and pitch were run considering the free surface mesh illustrated in Figure 38, which is a low order mesh with 50362 panels that covers a radius of $120 \mathrm{~m}$ around the floater. As shown in Figure 39, the impact of the free-surface is too small to justify the significantly larger computation time $^{2}$.

Hence, the QTFs that are considered hereafter are computed with the influence of the second-order potential (WAMIT .12 file) but without the free-surface forcing terms, and they are illustrated in Figure 40. The local peaks in the matrices around the natural frequency of heave $\left(\omega_{n 3}=0.641 \mathrm{rad} / \mathrm{s}\right)$ can be easily seen, but it is also remarkable the presence of peaks around the natural frequency of pitch $\left(\omega_{n 5}=0.299 \mathrm{rad} / \mathrm{s}\right)$ when looking

\footnotetext{
${ }^{2}$ The 119 frequency pairs took $38 \mathrm{~h}$ when accounting for the free-surface forcing terms, while the 4489 pairs of the full QTF took 11h without the free surface using the same computer.
} 
Figure 38 - Free surface mesh used to check the relevance of the free-surface forcing terms on the QTFs calculated with WAMIT.

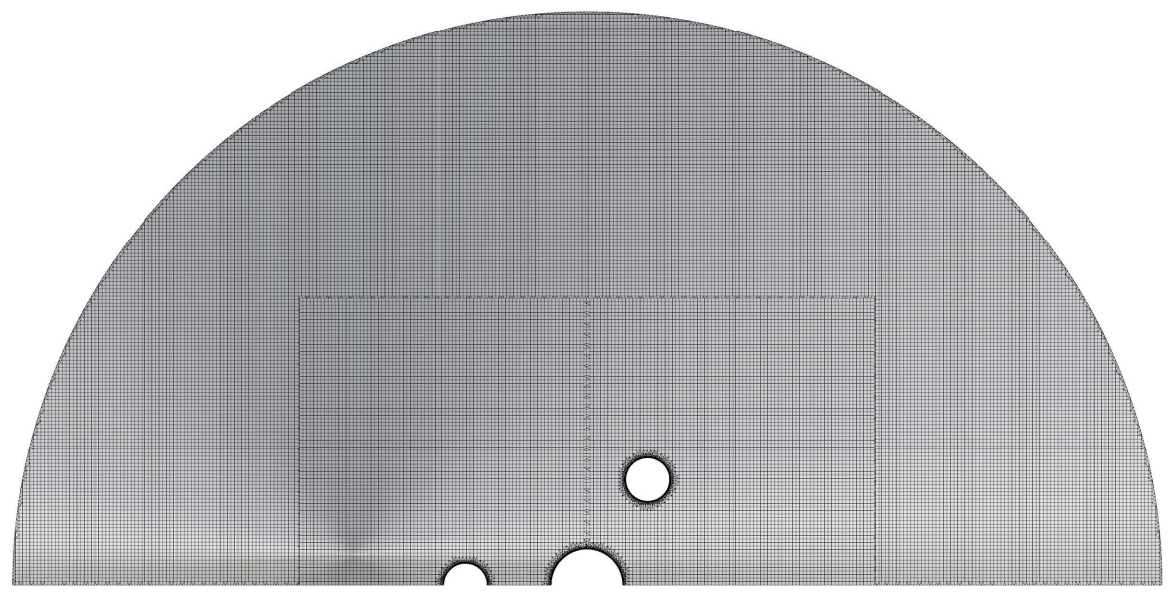

Figure 39 - Surge and pitch QTFs at the difference frequency corresponding to their natural frequencies 1) without the second-order potential, 2) with the secondorder potential, but neglecting the free-surface forcing terms and 3) with the complete second-order potential.
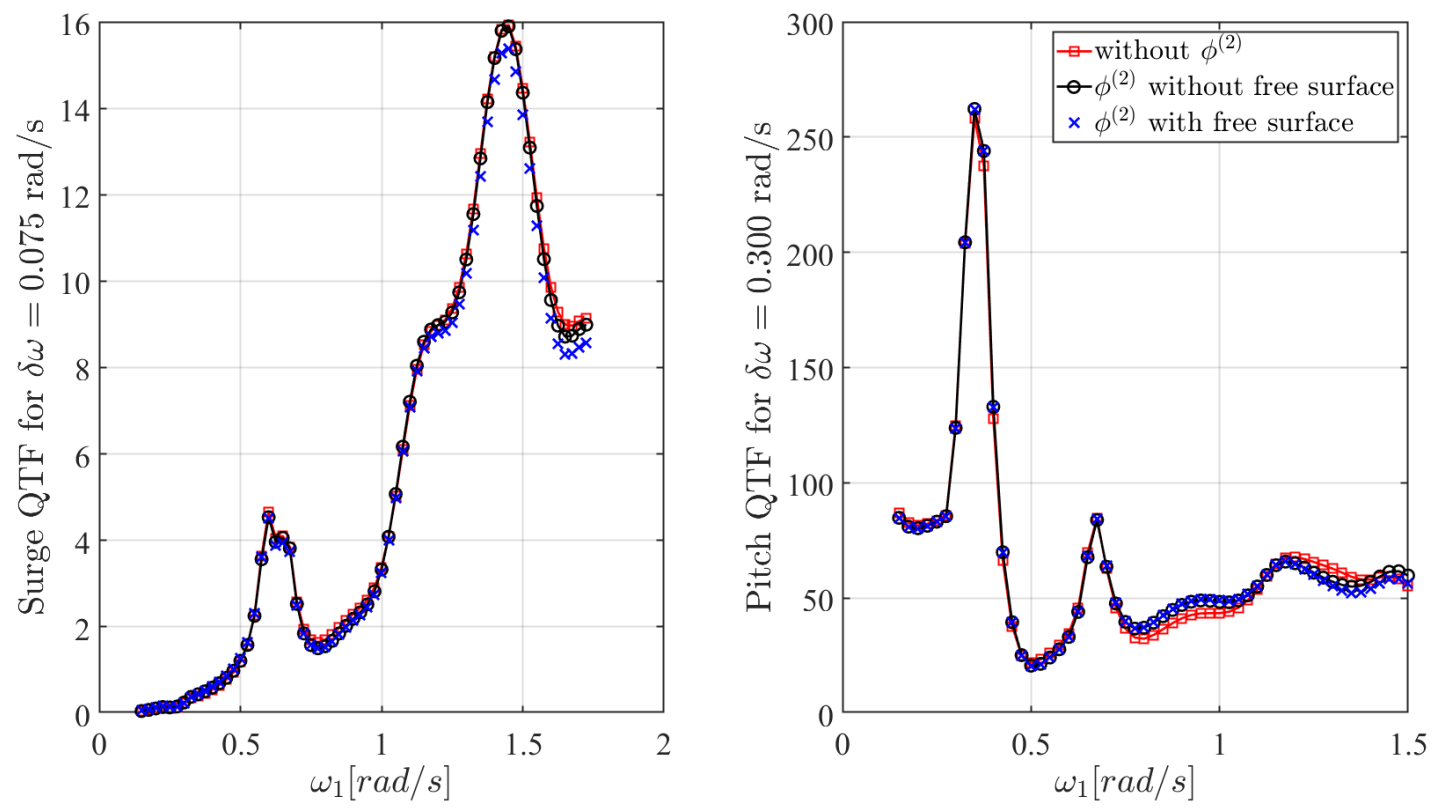

specifically at the diagonals that are the most relevant for the second-order heave and pitch loads.

Besides the results that are used as inputs to OpenFAST simulations, the motions calculated with WAMIT are also included in the comparisons considering white-noise and bichromatic waves. As in the latter the resonance frequency of surge is excited, an external linear damping term in surge direction, $B_{11}^{\text {ext }}$, is also needed, and the procedure that was followed to establish this value was already explained in Section 6.2.

For the simulations with nonzero wind speed, the aerodynamic loads induce additional damping and a mean displacement in both surge and pitch. Since the floater does 
Figure 40 - Color map of the nondimensional force/moment amplitude QTF matrices (left) and their diagonals around the corresponding natural frequency of motion for surge, heave and pitch (right). The frequency range with wave energy is indicated in blue.
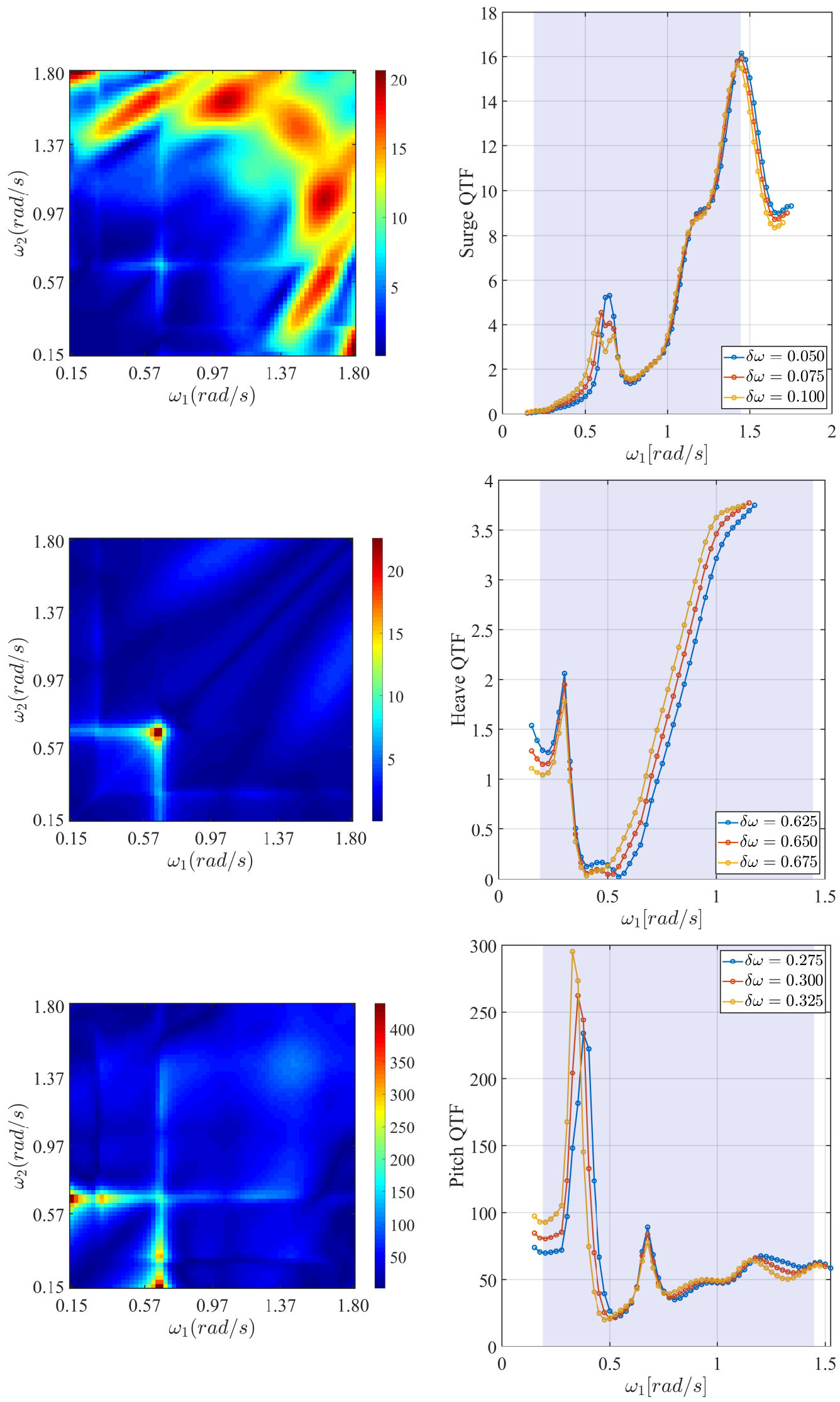
not have an active ballast system to counteract the aerodynamic thrust, the resulting mean inclination is not negligible, and it was shown by Amaral et al. (2021) (for the same floater, but with heave plates) that it is indeed significant for the computation of the hydrodynamic forces. Hence, these effects need to be considered when evaluating the frequency-domain coefficients. In order to model them, the results of the white-noise wave simulated in OpenFAST are used again, but this time considering the wind condition and rotor speed specified in Sections 6.1.1 and 6.1.3. The resulting mean displacements obtained with OpenFAST, shown later in this text to be equal to $-4.7 \mathrm{~m}$ in surge, $-5.1^{\circ}$ in pitch (with respect to the center of gravity) and negligible in heave, are considered in WAMIT by displacing the mesh, as illustrated in Figure 41, and by recalculating the mooring stiffness matrix at the displaced position:

$$
\mathbb{K}_{M, G, U_{w}}=\left[\begin{array}{cccccc}
6.56 \mathrm{e} 4 & 0 & -4.90 \mathrm{e} 3 & 0 & 4.72 \mathrm{e} 5 & 0 \\
0 & 7.38 \mathrm{e} 4 & 0 & -6.19 \mathrm{e} 5 & 0 & 1.05 \mathrm{e} 5 \\
-4.90 \mathrm{e} 3 & 0 & 1.04 \mathrm{e} 5 & 0 & -2.68 \mathrm{e} 5 & 0 \\
0 & -6.19 \mathrm{e} 5 & 0 & 1.15 \mathrm{e} 8 & 0 & 9.50 \mathrm{e} 6 \\
4.72 \mathrm{e} 5 & 0 & -2.68 \mathrm{e} 5 & 0 & 1.02 \mathrm{e} 8 & 0 \\
0 & 1.05 \mathrm{e} 5 & 0 & 9.50 \mathrm{e} 6 & 0 & 9.29 \mathrm{e} 7
\end{array}\right]
$$

with $U_{w}$ indicating that the matrix corresponds to the case with wind speed. The units are $\mathrm{kg}, \mathrm{m}$ and $\mathrm{s}$.

Figure 41 - Higher-order mesh for the simulations with wind (inclination of $-5.1^{\circ}$ around the global Y axis).

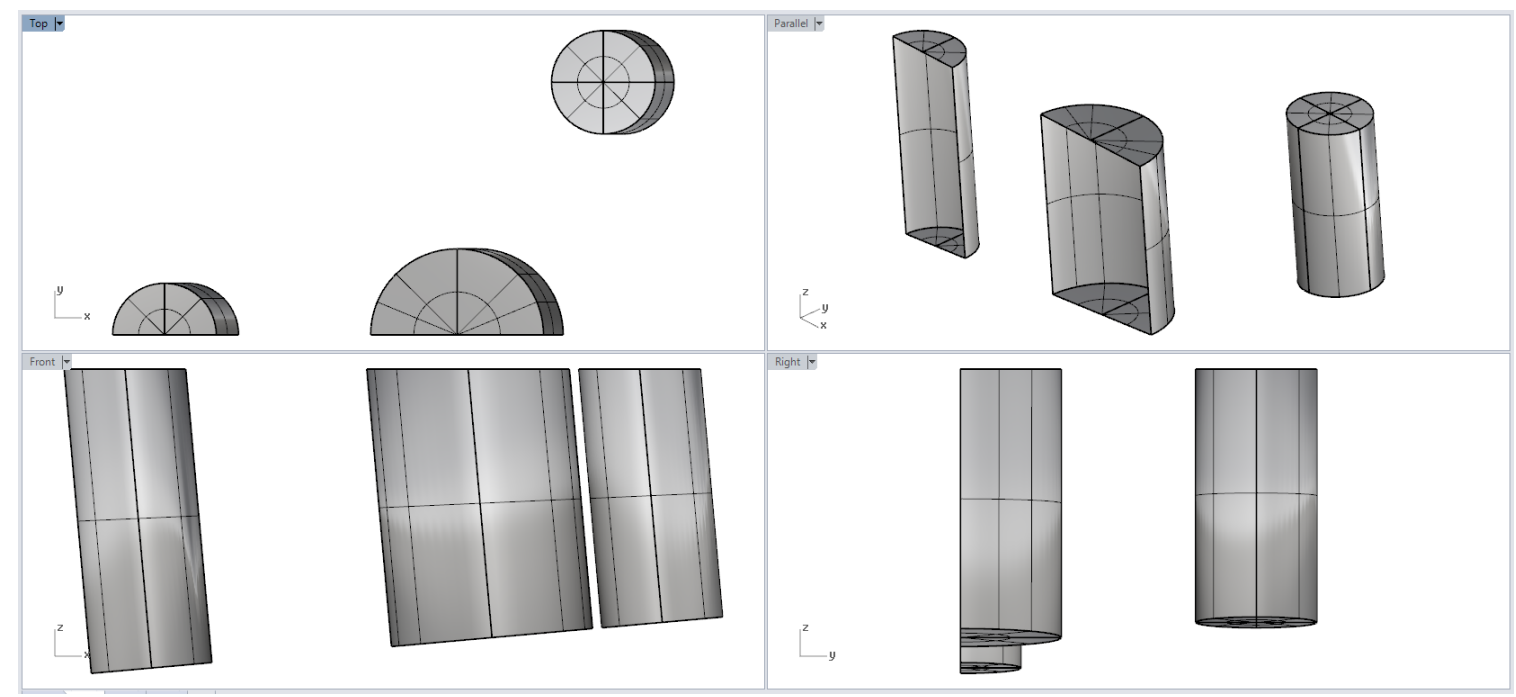

Since WAMIT is not used to assess second-order motions under the action of wind, only the aerodynamic damping in pitch is relevant (it is negligible in heave and the natural frequency of surge is outside the range of the first-order loads). A common approach for estimating such damping effects (KVITTEM; MOAN, 2015; HEGSETH; BACHYNSKI, 2019; SOUZA; HEGSETH; BACHYNSKI, 2020) is to use a damping factor obtained by 
linearizing the thrust, $F_{T}$, with respect to the wind speed, $U_{w}$, measured at the hub:

$$
B_{55}^{\text {aero }}=\frac{\partial F_{T}}{\partial U_{w}}\left(Z_{\mathrm{hub}}-Z_{G}\right)^{2}
$$

where the coefficient $\frac{\partial F_{T}}{\partial U_{w}}$ can be obtained from the steady-state response of the turbine as a function of the wind speed, given in Jonkman, Butterfield, et al. (2009) for the NREL $5 \mathrm{MW}$ reference turbine. For $U_{w}=7.8 \mathrm{~m} / \mathrm{s}$, it is equal to $8.75 \mathrm{e} 4 \mathrm{~N} . \mathrm{s} / \mathrm{m}$. Since the vertical distance between the hub and the center of gravity is $85.88 \mathrm{~m}$, the expression above results in $B_{55}^{\text {aero }}=6.4535$ e 8 N.m.s, which corresponds to about $23 \%$ of the critical pitch damping. As shown in Section 6.5.2, this value reproduces quite well the first-order pitch motion calculated in the time-domain simulations.

Figure 42 compares the nondimensional amplitude of the force/moment QTFs calculated with and without wind effects. For conciseness, only the diagonals that are the closest to the corresponding natural frequency of each degree of freedom are compared. Some differences can be observed in surge and pitch, specially around the peak of secondorder pitch moment related to the first-order pitch motion close to $\omega_{1}=\omega_{n 5}$, but the most remarkable change is the total absence of the peak of second-order heave force due to the first-order pitch motion. However, since slow heave is unimportant in this case, the results obtained considering either set of QTF matrices are similar, specially taking into account the uncertainties involved in the evaluation of motions induced by second-order forces. Nevertheless, the simulations with and without wind performed with OpenFAST consider the QTF matrices calculated in the corresponding condition.

Figure 42 - Nondimensional force/moment amplitude QTFs (for the most relevant diagonals) obtained with and without wind effects.
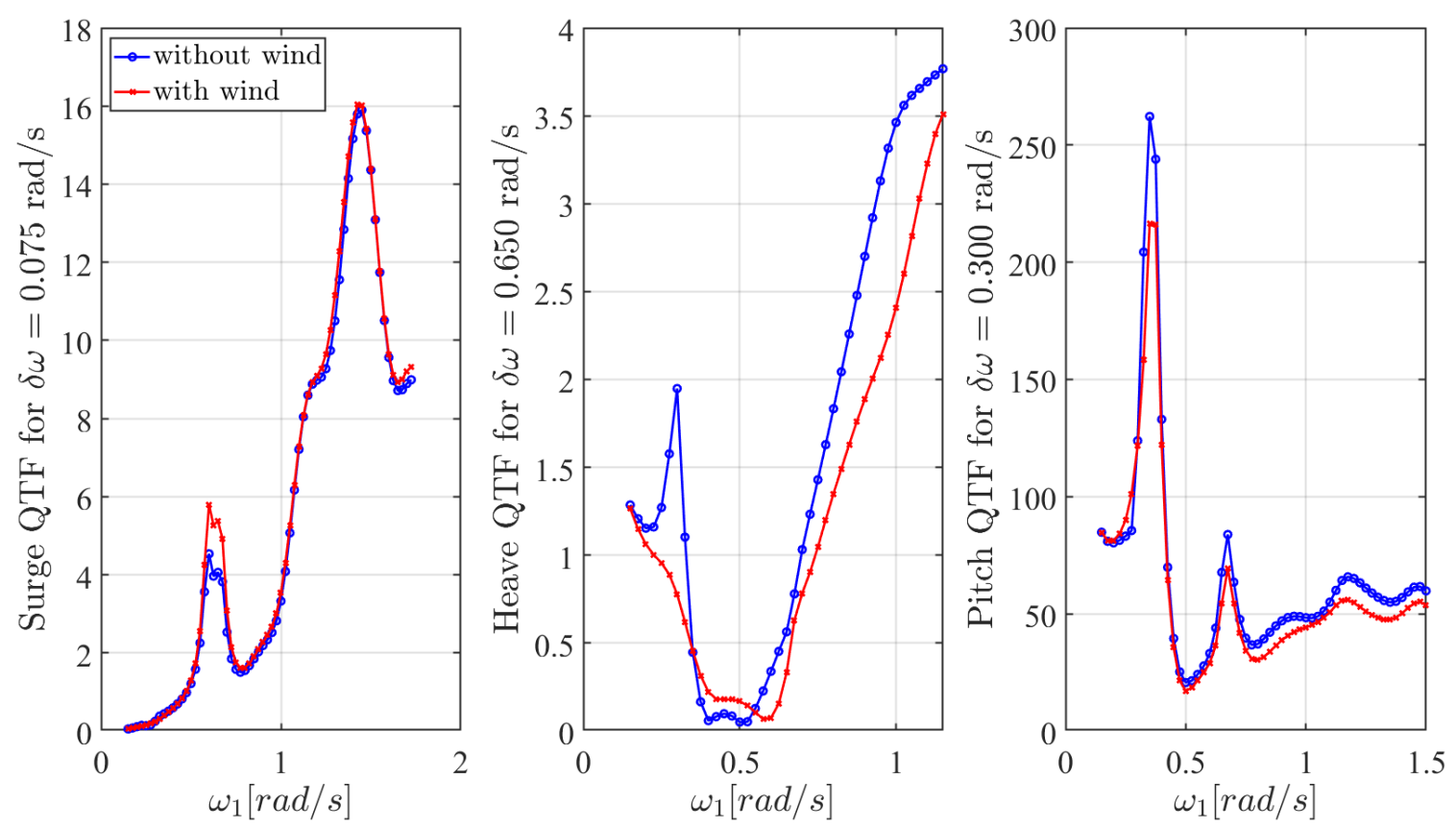


\subsubsection{METiS}

The floater was modeled in METiS by four cylinders, each representing one of the columns of the FOWT. As already discussed in Section 6.2, the added mass and drag coefficients are considered to be the same for all the columns, with their values obtained from the forced oscillation tests. The ones given in Table 19 are considered in general situations and the ones in Table 18 in the tests with bichromatic waves, with other values also adopted in some specific conditions, as indicated when suitable.

To determine the spatial discretization for integrating the forces along the cylinders, the horizontal drag force acting on the structure was compared for a regular wave with incidence $180^{\circ}$ and period of $3 \mathrm{~s}$, which is one second lower than the lowest period with wave energy in the test cases, considering three different spatial discretizations $\Delta z$. The results, given in Figure 43, show a difference of around $1 \%$ between the amplitudes calculated with the coarse $(\Delta z=1.00 \mathrm{~m})$ and the medium discretizations $(\Delta z=0.50 \mathrm{~m})$, and a difference of less than $0.1 \%$ between the medium and fine $(\Delta z=0.25 \mathrm{~m})$ discretizations. Based on that, a cylinder discretization of $\Delta z=0.50 \mathrm{~m}$ was adopted.

Figure 43 - Horizontal drag force calculated with METiS for different discretizations.

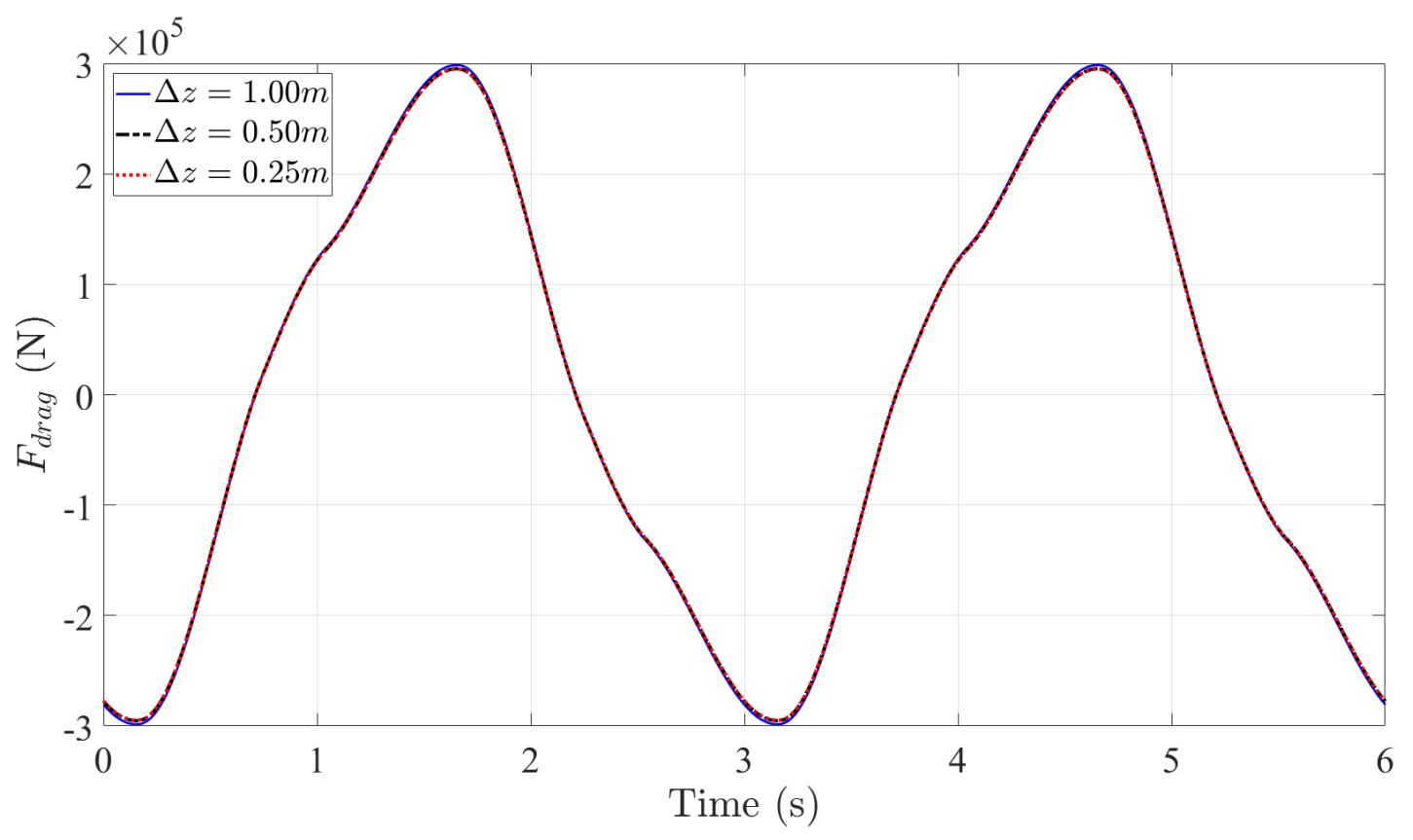

As the experimental sampling frequency is $100 \mathrm{~Hz}$ (model scale), the time series obtained in the experiments has a time discretization of $\Delta t_{e}=0.0894 \mathrm{~s}$. To ease the comparisons, this value was adopted as the time step for the METiS simulations as well. Other values were tested, namely $\Delta t_{e} / 2$ and $\Delta t_{e} / 10$, and the results, which are not reproduced here, corroborate that $\Delta t_{e}$ is small enough to avoid numerical instabilities.

Due to the low wave amplitudes considered in some of the tests, the quadratic drag alone is not sufficient to reproduce the damping involved in the first-order heave and 
pitch around their resonance frequencies. As already mentioned in Chapter 4, this is an expected downside of neglecting radiation effects, but it is specially troublesome in this work because it makes it hard to tell whether occasional differences in the second-order forces/motions around the peaks of first-order vertical motions are due to the force model or simply a consequence of getting the first-order motions wrong. For this reason, an external linear damping matrix was included in the METiS simulations:

$$
\mathcal{F}_{B_{\text {ext }}}=-\mathbb{B}_{\text {ext }} \dot{\boldsymbol{\xi}}
$$

with $\mathbb{B}_{\text {ext }, 33}=1.85 \mathrm{E}+05 \mathrm{~N} . \mathrm{s} / \mathrm{m}$ and $\mathbb{B}_{\text {ext }, 55}=2.66 \mathrm{E}+03 \mathrm{~N} . \mathrm{m} . \mathrm{s} / \mathrm{rad}$, which correspond to the potential damping calculated with WAMIT at the natural periods of heave and pitch, the only nonzero terms of the matrix. While $\mathbb{B}_{\text {ext, } 33}$ corresponds to $1.6 \%$ of the critical heave damping, the value of $\mathbb{B}_{\text {ext }, 55}$ is negligible in face of the critical pitch damping (which is equal to $2.37 \mathrm{E}+09$ N.m.s $/ \mathrm{rad}$ ), in such a way that it has an almost completely negligible impact on the results.

In order to guarantee that the waves analyzed in the numerical simulations were the same as the ones from the experimental tests, the records of wave elevation measured at the center of the tank during wave calibration were used as input to the software for each wave condition. As exemplified by the WHI01 wave condition, illustrated in Figure 44, the waves considered in the numerical simulations are indeed practically the same as the ones measured in the experiments.

Figure 44 - Comparison between the wave elevation measured in the experiments and the one simulated with METiS for the WHI01 wave condition.

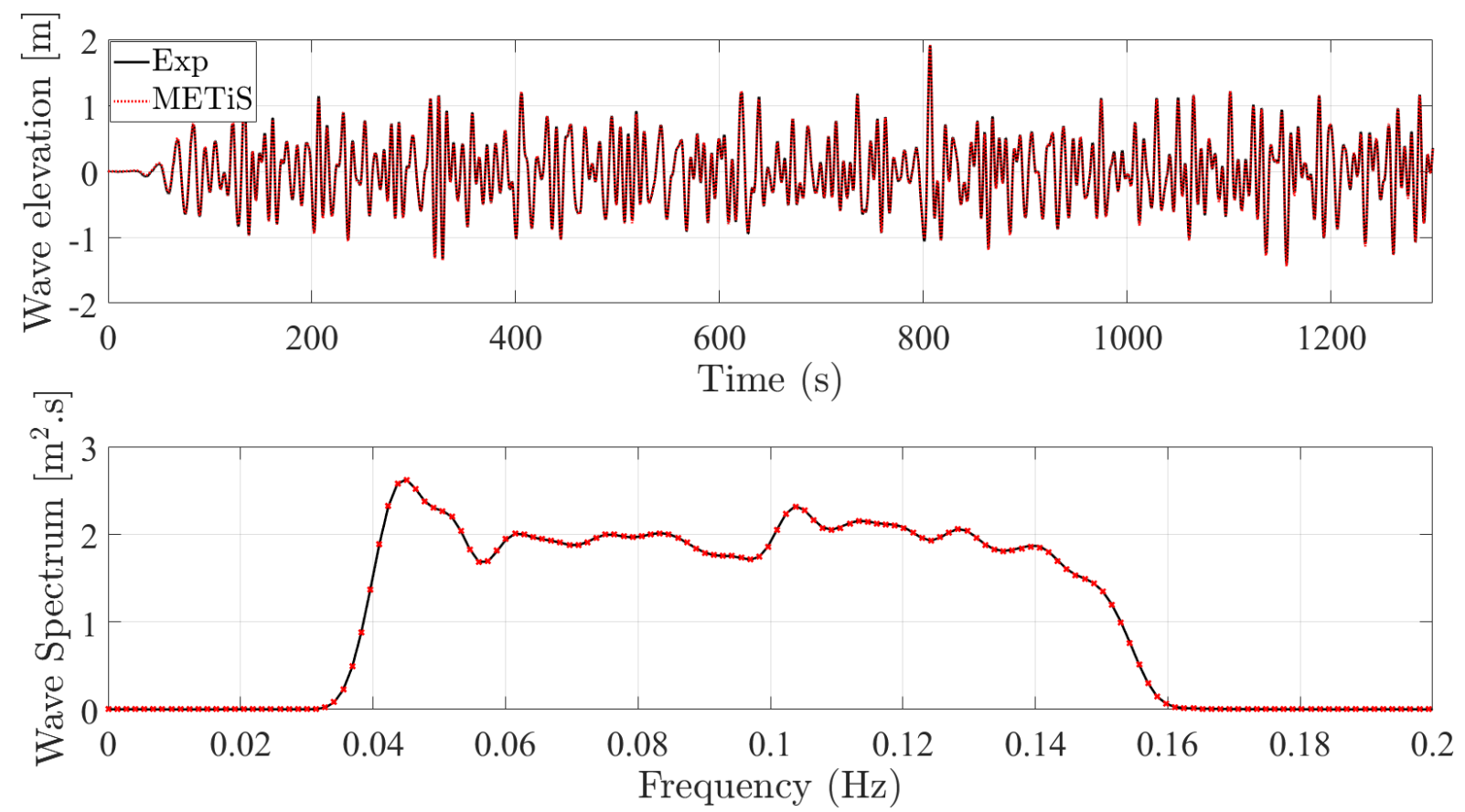

The mooring system is modeled using the same stiffness matrices considered 
in WAMIT (given by Equations 6.12 and 6.13) and a constant force vector equal to $\overline{\mathbf{F}}_{M}=-3.30 \mathrm{E}+06 \mathbf{E}_{3} \mathrm{~N}$ to account for the weight of the mooring lines.

For the simulations with the wind condition specified in Table 12, the rotor was kept rotating at a constant velocity of $9.07 \mathrm{rpm}$ and an initial displacement of $-4.8 \mathrm{~m}$ in surge and $-5.1^{\circ}$ (with respect to the center of gravity) in pitch, which are the mean displacements caused by the wind (the same that were used to displace the WAMIT mesh in Section 6.3.1), were imposed to the body in order to reduce transient effects.

\subsubsection{OpenFAST}

OpenFAST is a framework that couples several computational modules that deal with the different physical aspects of the coupled dynamics of floating wind turbines. The main modeling approaches that were adopted in each of them are summarized below.

The OpenFAST module responsible for solving the structural dynamics of the FOWT is called ElastoDyn, requiring as input the inertial and structural properties of the different subsystems (floater, tower, blades, etc). As this work is concerned only with the rigid body motions of the structure, only the six degrees of freedom (DoF) corresponding to surge, sway, heave, roll, pitch and yaw were kept active, meaning that the DoFs related to the flexibility of blades, tower and drivetrain and the ones related to the control of the nacelle, blades and generator were not included. Just like for METiS, the simulations with wind considered a constant rotor velocity of $9.07 \mathrm{rpm}$ and an initial displacement equal to the mean displacements caused by the wind $\left(-4.8 \mathrm{~m}\right.$ in surge and $-5.1^{\circ}$ in pitch) to reduce transient effects.

Hydrodynamics is modeled by HydroDyn considering the time series of wave elevation measured in the experiments. For the analyses presented in this text, wave loads were calculated as a combination of radiation/diffraction forces, taken into account using Cummins' approach (CUMMINS, 1962; OGILVIE, 1964) with frequency-domain coefficients computed with WAMIT, and the quadratic drag from Morison's equation with the same drag coefficients considered in METiS. One key aspect of this procedure is the fact that the forces and resulting motions of the body are not, in general, sinusoidal, in such a way that the inclusion of radiation loads (added mass and potential damping) requires the following equation:

$$
\mathcal{F}_{\text {rad }}(t)=-\mathbb{A}(\infty) \ddot{\boldsymbol{\xi}}(t)-\int_{0}^{t} \mathbb{R}(t-\tau) \dot{\boldsymbol{\xi}}(\tau) \mathrm{d} \tau
$$

where $\mathbb{A}(\infty)$ is the added mass matrix for $\omega=\infty$ and the kernel $\mathbb{R}$ is the velocity impulse function matrix, also known as memory function because it represents the radiation forces due to the past motions of the body. The convergence of this convolution integral has been determinant to the definition of the time step adopted in the simulations, as illustrated in 
Figure 45, which presents the results of numerical surge decays obtained with OpenFAST for three different time steps. It is clear from the time series that the largest time step, which was enough for the METiS simulations, leads to a significantly larger damping. The graph of a PQ analysis (which is better explained in the following section) on the right indicates that while the quadratic damping is practically the same for the three simulations, the linear damping, which is due to wave radiation and should be almost zero for this oscillation period, varies significantly with the choice of time step. In terms of percentage of the critical damping, the largest time step results in a linear damping of almost $2 \%$, while the time steps of $8.94 \mathrm{E}-3 \mathrm{~s}$ and $8.94 \mathrm{E}-4 \mathrm{~s}$ correspond to $0.3 \%$ and $0.05 \%$, respectively. Since the linear damping obtained with the medium time step is already quite small and the smallest time step is prohibitive due to the resulting long simulation time, the time step of 8.94E-3 s was adopted in all OpenFAST simulations presented hereafter.

Figure 45 - Numerical surge decays obtained with OpenFAST for three different time steps.
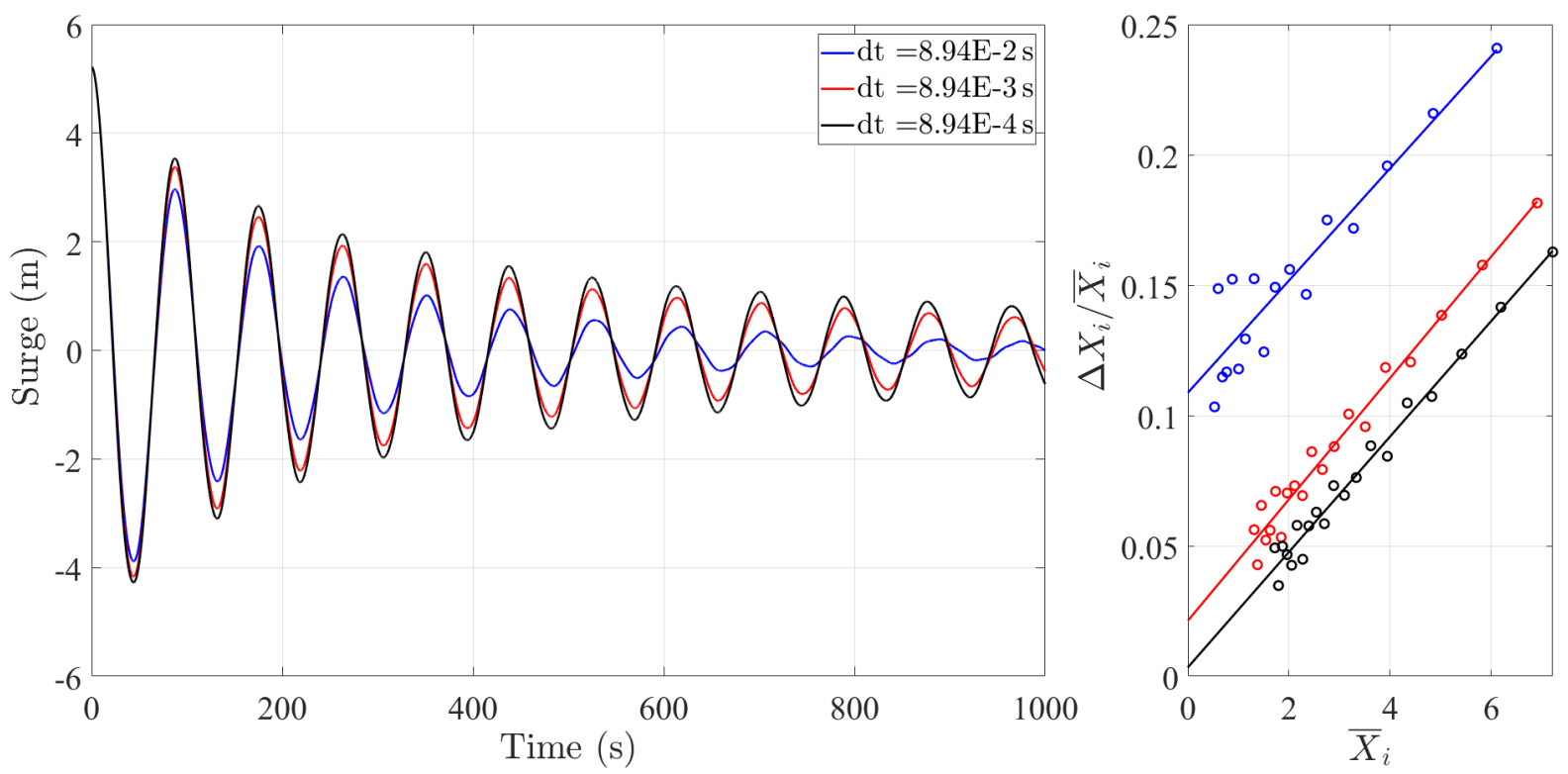

The mooring system is included using MoorDyn, which uses a lumped-mass formulation to model the dynamics of the mooring lines. The relevant inputs are the characteristics of the mooring lines already given in Table 11. Since METiS does not have the capability of properly modeling the mooring system yet, a second OpenFAST model was also analyzed by replacing MoorDyn by a linear stiffness matrix and constant force vector equal to the ones considered in METiS, with the objective of verifying whether the discrepancies observed in the results are due to differences in the hydrodynamic models or to the dynamics of the moorings. Moreover, some simulations with the complete mooring system with their drag coefficients set to zero are also analyzed.

For the simulations with wind, the InflowWind module was used to input the wind profile described in Table 12, while AeroDyn was employed to solve the aerodynamic forces 
on the rotor with Blade Element Momentum Theory. Since aerodynamic effects due to the tower, such as tower shadow or the aerodynamic loads on the tower itself, are not modeled in METiS yet, they were not included in AeroDyn either.

\subsection{Decay tests results}

This section compares the results of numerical decay tests in surge, heave and pitch performed with METiS and OpenFAST with the ones obtained in the experimental campaign. They were performed by imposing an initial displacement to the body at the desired DoF and then letting it oscillate freely in otherwise calm waters. In the experiments, at least five repetitions were performed for each DoF, and the experimental results presented in the tables below are the mean of the values measured in these repetitions. Due to the already mentioned problems regarding wind emulation in the experiments, decays with wind are analyzed only numerically.

The data obtained from the decays are time series of motion of the DoF of interest, as illustrated in the left part of Figure 46, for the decays without wind, and Figure 47, for the ones with wind (the right side of these figures will be explained ahead). One of the main results that can be obtained from these time series are the periods of motion of each DoF, $T_{n, \alpha}$, which can be readily calculated by the mean time difference between consecutive peaks. The results, given in Table 21, show that the natural periods obtained with the software match very well the experimental values, with the largest differences being lower than $2 \%$. When wind effects are included, the aerodynamic forces lead to a small increment of the surge natural period, while the pitch motion is attenuated so quickly that only three peaks were identified (the rest of the motion being due to coupling with surge), and this is why the corresponding row is missing from the table.

Table 21 - Natural periods from decay tests.

\begin{tabular}{ccccc}
\hline & & Exp. (s) & METiS (s) & OpenFAST (s) \\
\hline & Surge & 86.3 & 87.3 & 88.0 \\
& Heave & 9.8 & 9.8 & 9.7 \\
& Pitch & 21.0 & 21.1 & 20.8 \\
\hline & Surge & - & 89.3 & 89.5 \\
& Heave & - & 9.8 & 9.7 \\
\hline
\end{tabular}

As can be seen in Figure 47, the mean displacements induced by the wind predicted by METiS and OpenFAST, which are also listed in Table 22, are not exactly the same. Nevertheless, since the differences are below $3 \%$, this is considered a good agreement.

Another important property that can be obtained from the decays is damping, 
Figure 46 - Results obtained from the decay tests without wind.
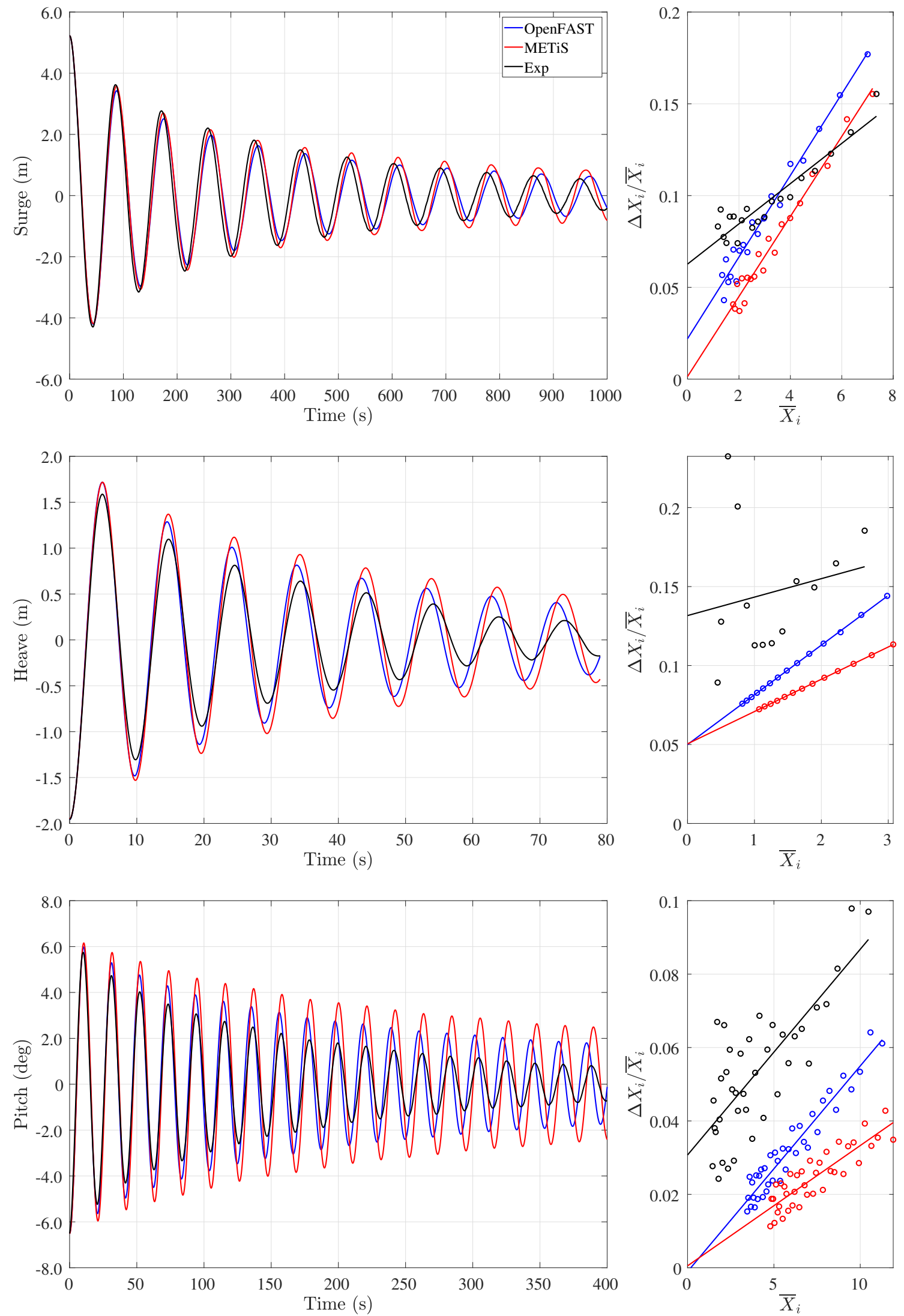
Figure 47 - Results obtained from the decay tests with wind and rotor speeds equal to $7.8 \mathrm{~m} / \mathrm{s}$ and $9.07 \mathrm{rpm}$. Due to the few peaks observed in pitch, the corresponding PQ analysis must be ignored.
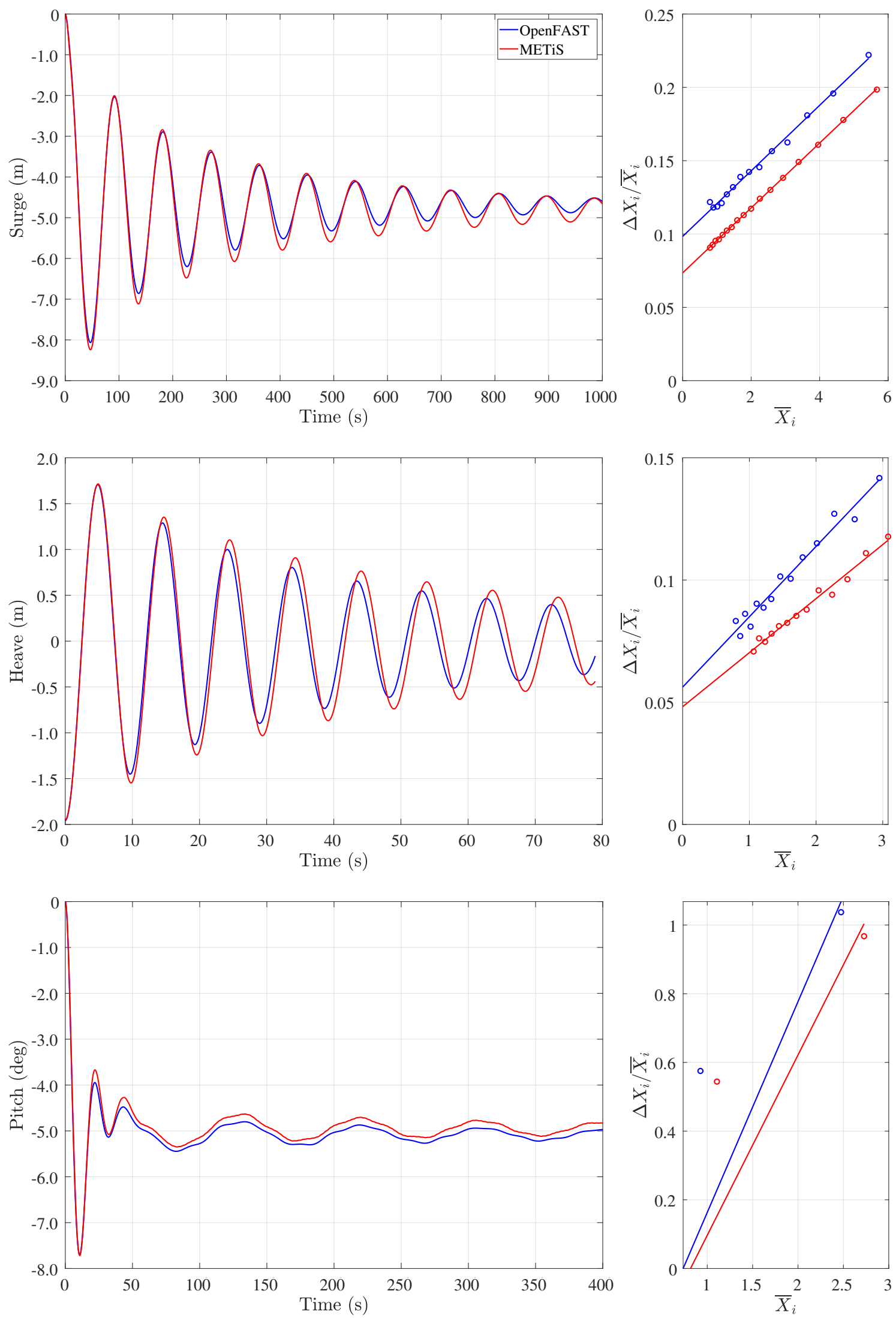
Table 22 - Mean displacements induced by the wind.

\begin{tabular}{lcc}
\hline & OpenFAST & METiS \\
\hline Surge $(\mathrm{m})$ & -4.70 & -4.81 \\
Pitch $\left({ }^{\circ}\right)$ & -5.08 & -4.94 \\
Heave $(\mathrm{m})$ & \multicolumn{2}{c}{$<0.01$} \\
\hline
\end{tabular}

assumed to be simply the superposition of linear and quadratic terms:

$$
F_{\text {damp }}=B_{\alpha, \text { lin }} \dot{\xi}_{\alpha}+B_{\alpha, \text { quad }} \dot{\xi}_{\alpha}\left|\dot{\xi}_{\alpha}\right|
$$

which also assumes that the motions at the DoF $\alpha$ are decoupled from the others. The coefficients $B_{\alpha, \text { lin }}$ and $B_{\alpha, \text { quad }}$ are obtained using the classical PQ-method (VAN DER VEGT, 1984 apud BURMESTER et al., 2020) ${ }^{3}$, which is based on calculating the ratio between the decrement of successive amplitudes and their mean amplitude, $\Delta X_{i} / \bar{X}_{i}$, where $\Delta X_{i}=X_{i+1}-X_{i}, \bar{X}_{i}=0.5\left(X_{i+1}+X_{i}\right)$ and $X_{i}$ is the amplitude of motion of the peak $i$. The relative decrement $\Delta X_{i} / \bar{X}_{i}$ is then plotted as a function of $\bar{X}_{i}$, and a line is fitted through these points, as illustrated in the right side of Figures 46 and 47 . The interception of this line with the ordinate and its slope, denoted respectively as $p_{\alpha}$ and $q_{\alpha}$, are related to the damping coefficients as follows:

$$
\begin{aligned}
B_{\alpha, \text { lin }} & =2 p_{\alpha} \frac{M_{\alpha}}{T_{n, \alpha}} \\
B_{\alpha, \text { quad }} & =\frac{3}{8} q_{\alpha} M_{\alpha}
\end{aligned}
$$

where $M_{\alpha}=\mathbb{M}_{\alpha \alpha}+\mathbb{A}_{\alpha \alpha}\left(\omega_{n, \alpha}\right)$, thus including both body inertia and added mass.

In order to assess the impact of coupling effects, additional OpenFAST and METiS models were run with all DoFs, except for the one of interest, disabled. Besides that, this uncoupled version of the OpenFAST decays was also simulated without drag due to the moorings, which is useful to evaluate their impact on the damping levels. The results obtained in these conditions are given in Figure 48 and included in the tables discussed ahead. It is interesting to note that, in this case, the points obtained with the PQ method follow the theoretical line quite well (except for the pitch motion in OpenFAST, for reasons still unknown), showing that the spreading observed in Figure 46 is due to coupling effects.

Concerning the linear damping, given in Table 23 as a fraction of the critical damping, $\zeta_{\alpha}$, the first remarkable point is that the values measured in the experiments are larger than the radiation damping predicted with WAMIT, indicating that viscous effects also affect the linear damping coefficients. For instance, the experimental surge decay provides $\zeta_{1}=1.0 \%$, while WAMIT predicts an almost null value (it is worth reminding the issue regarding the convergence of the memory integral in OpenFAST pointed out in

\footnotetext{
${ }^{3}$ van der Vegt, J. J. Slinger gedrag van schepen. KIVI-Lecture on Seakeeping, 1984.
} 
Figure 48 - PQ analysis of the decoupled decay simulations neglecting drag due to the moorings. The experimental results are the same from the previous graphs.
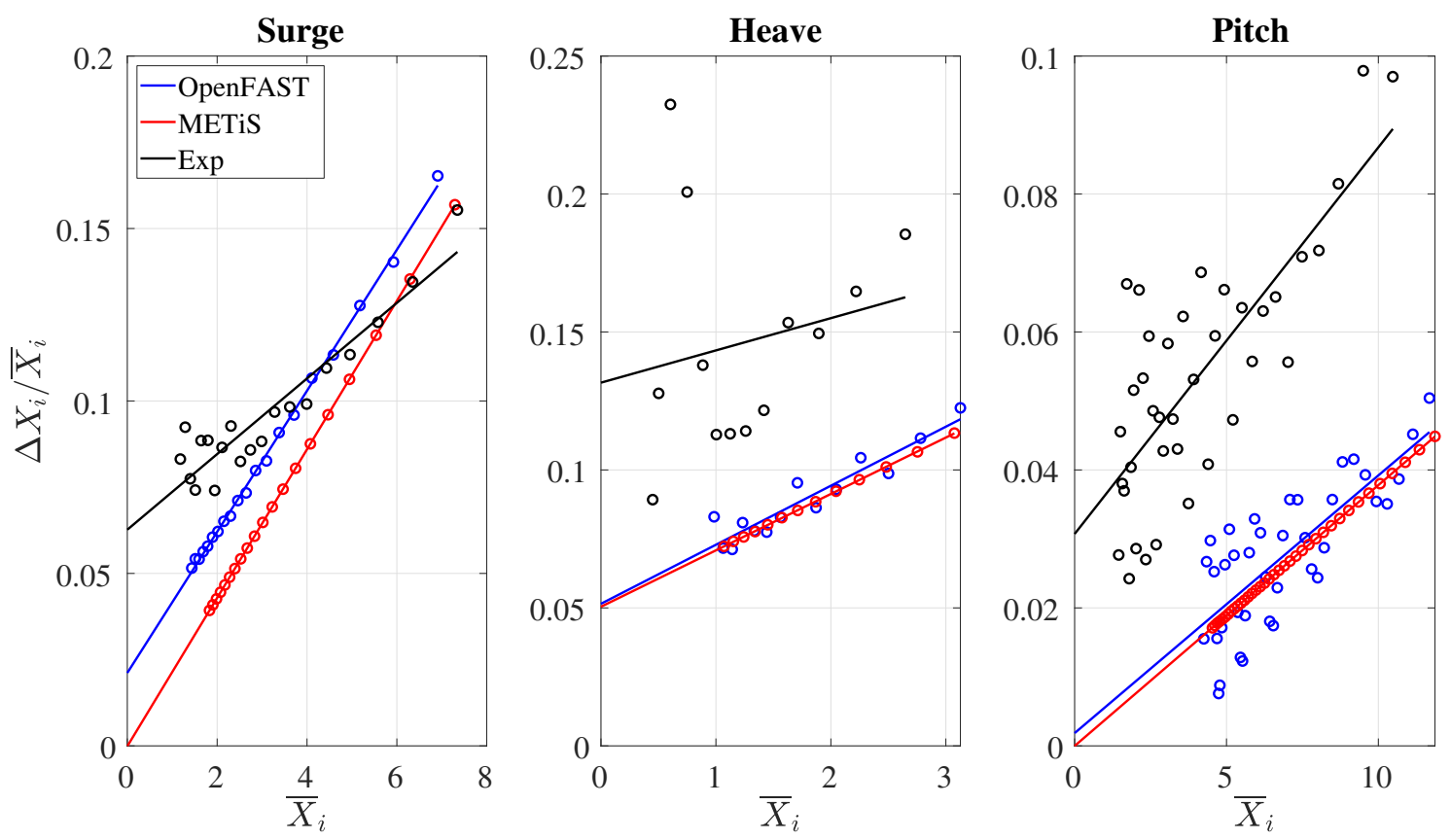

Section 6.3.3, which makes the linear damping in surge larger than it should). Though this could be an issue for small motions, for which the linear damping is significant, it should be negligible in face of the quadratic damping for large motions. Neither coupling nor mooring effects impacted the linear damping obtained in the numerical simulations in any of the DoFs.

Table 23 - Ratio between linear $\left(B_{\operatorname{lin}}\right)$ and critical damping obtained from decays.

\begin{tabular}{|c|c|c|c|c|c|c|}
\hline & & \multirow{2}{*}{ Exp. (\%) } & \multicolumn{2}{|c|}{ METiS (\%) } & \multicolumn{2}{|c|}{ OpenFAST (\%) } \\
\hline & & & Coupled & Decoup. & Coupled & Decoup. $\left(C_{D}^{\text {moor }}=0\right)$ \\
\hline \multirow{3}{*}{ 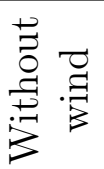 } & Surge & 1.0 & 0.0 & 0.0 & 0.3 & 0.3 \\
\hline & Heave & 2.1 & 0.8 & 0.8 & 0.8 & 0.8 \\
\hline & Pitch & 0.5 & 0.0 & 0.0 & 0.0 & 0.0 \\
\hline \multirow{2}{*}{ 霛 } & Surge & - & 1.2 & - & 1.6 & - \\
\hline & Heave & - & 0.8 & - & 0.9 & - \\
\hline
\end{tabular}

Once wind is included, the linear surge damping increases about the same amount in both software, i.e. around $1.2 \%$, while heave changes only slightly. As already noted, the pitch motion is attenuated after only three cycles, which is coherent with the linear damping of $23 \%$ estimated for the WAMIT model in Section 6.3.1 with Equation 6.14.

The values obtained for the quadratic damping are given in Table 24. Regarding surge, the ones computed with both METiS and OpenFAST are very close, meaning that moorings do not induce significant damping in this DoF. However, it corresponds to twice the value measured in the experiment, so that the adopted value of $C_{D}=1.00$ is also around 
twice the one that would be necessary to match the experimental decay. Nevertheless, given that the drag coefficient is dependent on the amplitude of motion, as shown by the forced oscillation tests, it is reasonable to conclude that this superposition of a constant linear and quadratic damping coefficients is too simple to properly reproduce the experiments. In fact, the quadratic drag is expected to change between the beginning of the decay test, where motions are large, and its end, for which motions are far smaller. For instance, if only the first 3 points of the graph in the right of Figure 46 were used to conduct the PQ analysis, the experimental quadratic damping would result in $1.0225 \mathrm{E}+05 \mathrm{~N} . \mathrm{s}^{2} . \mathrm{m}^{-2}$, hence within about $5 \%$ of the value calculated from the numerical simulations. Thus, even though the values shown in Table 24 are different, this is actually a matter of tuning $C_{D}$ based on the amplitude of motion. This is confirmed in Section 6.6, where drag coefficients chosen based on the amplitudes of motion result in good estimations of the experimental slow surge.

Table 24 - Quadratic damping $\left(B_{\text {quad }}\right)$ from decay tests. Units for surge and heave are $\mathrm{N} . \mathrm{s}^{2} \cdot \mathrm{m}^{-2}$, while for pitch it is N.m.s ${ }^{2} \operatorname{rad}^{-2}$.

\begin{tabular}{|c|c|c|c|c|c|c|}
\hline & & \multirow{2}{*}{ Exp. } & \multicolumn{2}{|c|}{ METiS } & \multicolumn{2}{|r|}{ OpenFAST } \\
\hline & & & Coupled & Decoup. & Coupled & Decoup. $\left(C_{D}^{\text {moor }}=0\right)$ \\
\hline \multirow{3}{*}{ 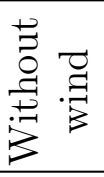 } & Surge & $5.35 \mathrm{E}+04$ & $1.07 \mathrm{E}+05$ & $1.05 \mathrm{E}+05$ & $1.09 \mathrm{E}+05$ & $1.00 \mathrm{E}+05$ \\
\hline & Heave & $3.93 \mathrm{E}+04$ & $6.88 \mathrm{E}+04$ & $6.88 \mathrm{E}+04$ & $1.06 \mathrm{E}+5$ & $7.20 \mathrm{E}+04$ \\
\hline & Pitch & $4.50 \mathrm{E}+08$ & $2.63 \mathrm{E}+08$ & $3.04 \mathrm{E}+08$ & $4.50 \mathrm{E}+8$ & $3.00 \mathrm{E}+08$ \\
\hline \multirow{2}{*}{ 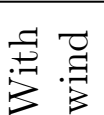 } & Surge & - & $1.08 \mathrm{E}+05$ & - & $1.09 \mathrm{E}+05$ & - \\
\hline & Heave & - & $7.44 \mathrm{E}+04$ & - & $9.68 \mathrm{E}+04$ & - \\
\hline
\end{tabular}

The same observation applies for heave, for which the quadratic drag obtained with both software is more than $75 \%$ larger than the experimental one. But in this case, the comparison between OpenFAST with and without drag due to the moorings shows that this component is significant to heave motion, increasing the quadratic drag by almost $50 \%$. When this component is neglected, $B_{\text {quad }}$ calculated with METiS and OpenFAST are within $5 \%$ of each other.

For pitch, the agreement between the quadratic drag calculated with OpenFAST and the experiments is excellent, as they are practically the same. The moorings have the same impact observed for heave, increasing the quadratic damping by $50 \%$ and, once again, if mooring drag is neglected, the quadratic damping obtained with METiS and OpenFAST are very close.

Finally, Table 24 shows that while the quadratic surge damping is unaffected by the wind, it has an opposite effect on the quadratic heave damping evaluated with the software, which is increased in METiS and reduced in OpenFAST. The reason for this contradictory behavior between them is not clear yet. 


\subsection{First-order results}

\subsubsection{First-order forces due to regular waves}

Before verifying first-order body motions, it is useful to check the agreement of the forces predicted with the different software. Since OpenFAST imports the potential part of the hydrodynamic forces from WAMIT, this subsection compares the first-order forces calculated using METiS with the ones computed with WAMIT (.3 file). As METiS is a time-domain code, the first-order forces were obtained by running independent simulations of the floater under the action of regular waves with periods ranging from $3 \mathrm{~s}$ to $30 \mathrm{~s}$ and constant amplitude equal to $1.0 \mathrm{~m}$.

Figure 49 compares the nondimensional force/moment amplitudes in surge, heave, pitch and yaw calculated with WAMIT and METiS for a wave incidence of $210^{\circ}$, with the mean column radius $\bar{R}$ taken as length scale. For conformity with the requirements of OpenFAST, the moments are given with respect to the intersection of the axis of the center column with the mean waterline. Results in sway and roll are not included because they are qualitatively similar to those of surge and pitch due to the triangular shape of the body. In order to illustrate the impact of the choice of $C_{a}$ on the forces predicted by METiS, the results obtained with a variation of $10 \%$ around the base value $C_{a}=0.82$ are shown in the graphs as vertical bars for the METiS results. Since the force variation, $\Delta F_{X}$, due to a given $\Delta C_{a}$ is simply:

$$
\Delta F_{X}=1+\frac{\Delta C_{a}}{1+C_{a}}
$$

the bars represent a constant variation of roughly $4 \%$ around the mean value in this case. The same effect is observed for pitch and yaw, as the only difference is the lever arm, while heave remains unchanged because it is not impacted by the value of $C_{a}$.

The results obtained with both software show a good agreement for waves with period above $6 \mathrm{~s}$, which is about the threshold of $\lambda / D>5$ usually adopted for Morison's equation when taking the mean diameter of the columns, $\bar{D}=10.5$, as the characteristic length. The DoF with the worst agreement is pitch, for which the moment calculated with METiS shows a difference of about $15 \%$ with respect to WAMIT even in long waves. The reason, which was already pointed out in Chapter 5 , is that the added mass coefficient is supposed the same along the whole length of the columns, which is specially troublesome for pitch because this assumption is worse in the regions where the lever is larger. $M_{Y}$ presents a peak and a valley around $4.0 \mathrm{~s}$ and $5.4 \mathrm{~s}$ that correspond approximately to wave lengths equal to the distance between the center of the side columns and the center of the main column $(\approx 25.4 \mathrm{~m})$ and equal to the distance between the centers of two side columns $(\approx 44.0 \mathrm{~m})$, and even though METiS provides a peak and a valley in the same periods, it fails to match the amplitude because here scattering effects are significant. These are the 
Figure 49 - Nondimensional force/moment amplitude in surge, heave, pitch and yaw calculated with WAMIT and METiS for a wave incidence of $210^{\circ} . \bar{R}=5.25 \mathrm{~m}$ is the mean column radius.
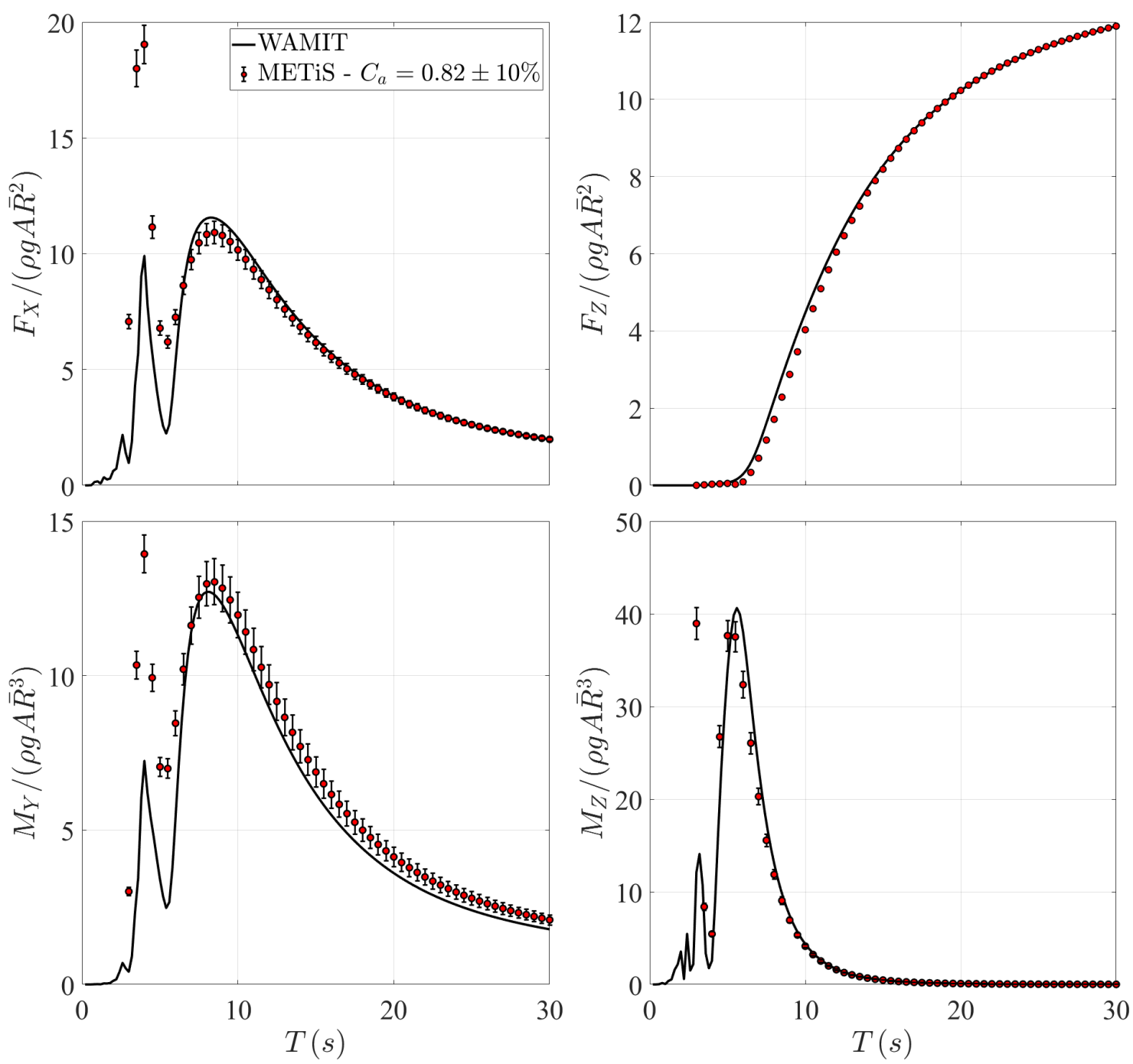

only results presented in this work that consider a wave incidence different from $180^{\circ}$, and the objective was to show that the first-order yaw moment also presents an overall good agreement in long waves.

Since the simulations considering the action of the wind take into account the resulting mean inclination of the hull, it is important to assess the impact of this inclination on the hydrodynamic forces. For such, Figure 50 compares the loads acting on the floater in its undisplaced position with the ones considering the mean displacement due to the wind, for waves with amplitude $1.0 \mathrm{~m}$ and incidence of $180^{\circ}$, hence only surge, heave and pitch are relevant. The results change very little, with the most remarkable difference being the appearance of a peak in the heave force around 5.4s (wave length corresponding to the distance between the centers of two side columns) when the floater is inclined due to the wind, which is present in both METiS and WAMIT. The overall conclusions, however, remain the same. 
Figure 50 - Nondimensional force/moment amplitude in surge, heave and pitch acting on the floater considering its undisplaced position and the mean displacement induced by the wind for a wave incidence of $180^{\circ}$.
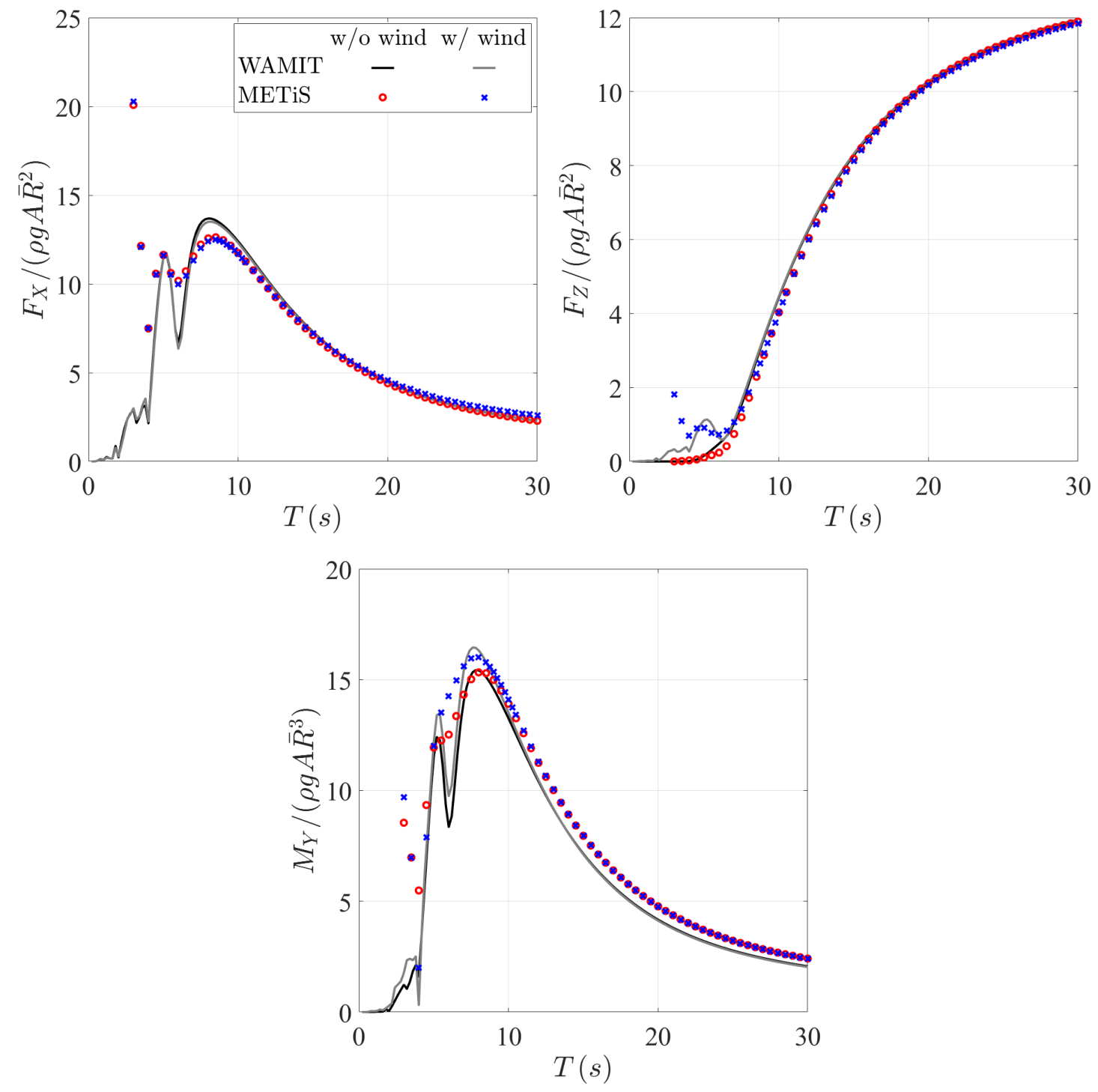

\subsubsection{First-order motion RAOs}

This section aims at comparing the motion Response Amplitude Operators (RAOs) computed with WAMIT, OpenFAST and METiS with the ones measured in the experiments. While these are directly output by WAMIT for being a frequency-domain code, the experimental and numerical RAOs from OpenFAST and METiS are evaluated from the response of the floater under the action of the WHI01 wave. The complex RAO, $Q(\omega)$, of an arbitrary response of the body, $q(t)$, due to waves with elevation $\eta(t)$ (measured at a reference point) can be computed as follows:

$$
Q(\omega)=\frac{S_{\eta q}(\omega)}{S_{\eta}(\omega)}
$$

where $S_{\eta}$ is the wave spectrum and $S_{\eta q}$ the cross power spectral density between $q$ and $\eta$, which are calculated by the Fourier transform of the cross-correlation function, $R_{\eta q}(t)$, of 
the signals:

$$
S_{\eta q}(\omega)=\int_{-\infty}^{\infty} R_{\eta q}(\tau) e^{-i \omega \tau} \mathrm{d} \tau
$$

with $R_{\eta q}$ defined as:

$$
R_{\eta q}(t)=\int_{-\infty}^{\infty} \eta(\tau) q(t+\tau) \mathrm{d} \tau
$$

and $S_{\eta}=S_{\eta \eta}$ simply a particular case of these relations.

Equation 6.21 is valid for any wave spectrum, but it clearly does not apply to frequencies where $S_{\eta}(\omega)=0$. Hence, to reduce distortions, the best alternative is to use a wave spectrum with a constant amplitude within the range of frequencies of interest, namely a white-noise wave. In reality, though, it is impossible to avoid some amplitude variation, as shown by the spectrum of the wave WHI01 that was already illustrated in Figure 44. Nevertheless, it is still a very practical way to evaluate the first-order RAOs. There are several ways to numerically compute the spectrum densities, and this work uses Welch's averaged, modified periodogram method (PRESS et al., 1992) with a Hamming window with $50 \%$ overlap.

A comparison of the RAOs predicted by the numerical methods and those derived directly from the experimental motion records, for the tests without wind effects, is given in Figure 51. The surge motion obtained with both METiS and OpenFAST match the experiments quite well. In heave, METiS provides results that are very close to the experiments, while OpenFAST presents a peak that is about $20 \%$ larger. Though the peak of heave motion calculated with WAMIT is even larger, it is sufficiently close to the experiments for the calculation of the second-order forces, given that a predictive approach is sought instead of calibrating coefficients until the results match the experiments.

Once again, pitch is the DoF for which METiS presents the largest discrepancies. While the results obtained with OpenFAST and WAMIT are quite good, even though the latter has a somewhat narrower peak that is slightly displaced to the left, METiS predicts a peak that is about $20 \%$ larger than the experiment, which at first sight may seen to be caused by the lack of damping due to the moorings, since it was shown to have a large impact on the damping measured from pitch decay tests. However, it was assessed that the damping introduced by the moorings in OpenFAST is very small in this case, in such a way that the larger pitch peak obtained with METiS is probably due to the overestimation of the pitch moment observed in the previous section. Nevertheless, as METiS is supposed to be a fast and simple tool for early design stages, this difference is considered acceptable.

Both surge and heave RAOs are barely affected by the aerodynamic forces due to the wind, at least regarding first order motions. On the other hand, the peak of pitch 
Figure 51 - Surge, heave and pitch RAOs (with respect to the CoG) for a wave incidence of $180^{\circ}$.
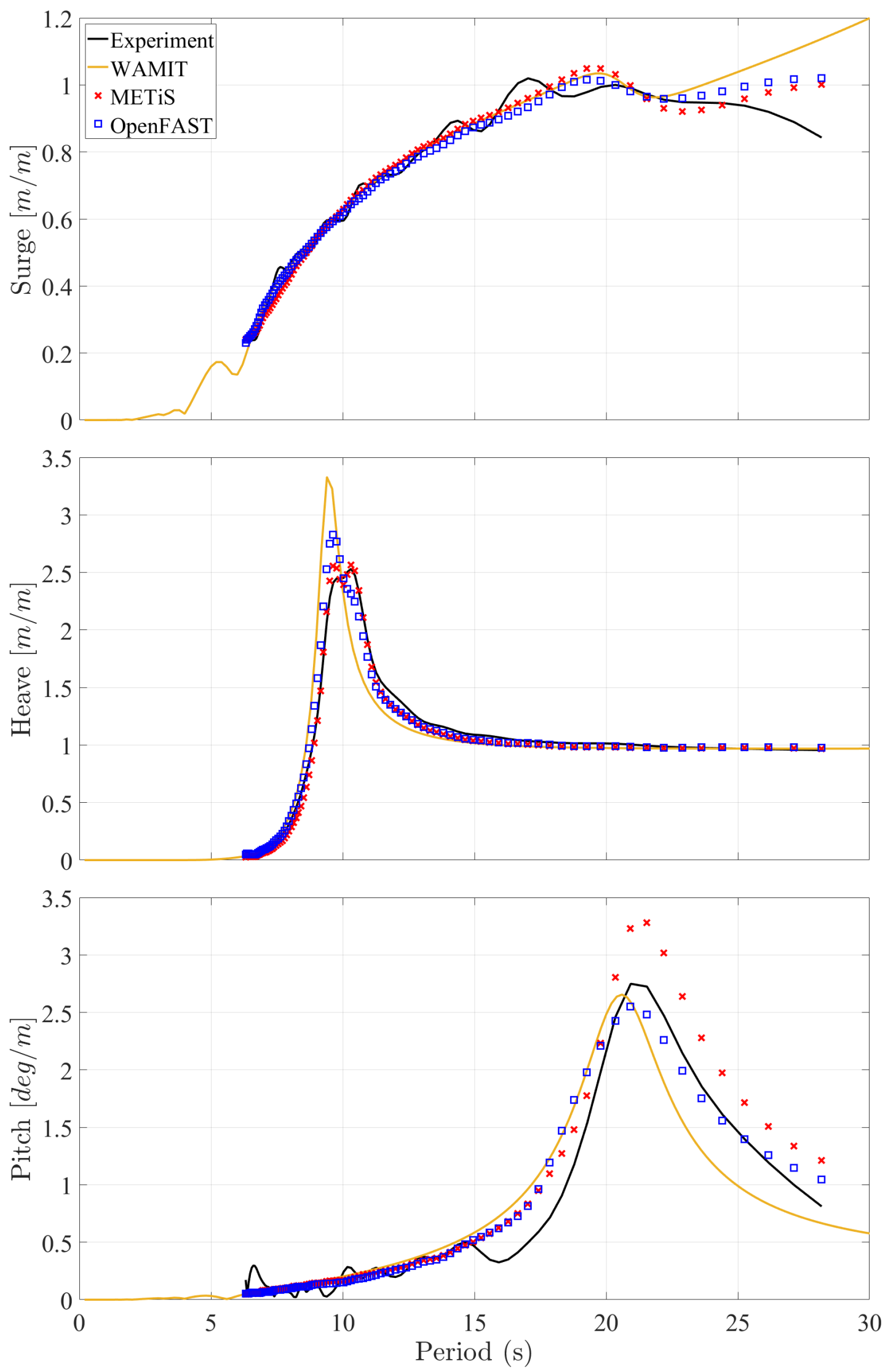
motion is completely flattened when wind is included in the analysis, as illustrated in Figure 52, and the results predicted by the software are similar.

Figure 52 - Pitch RAO (with respect to the CoG) for an incidence of $180^{\circ}$ accounting for aerodynamic thrust on the rotor.

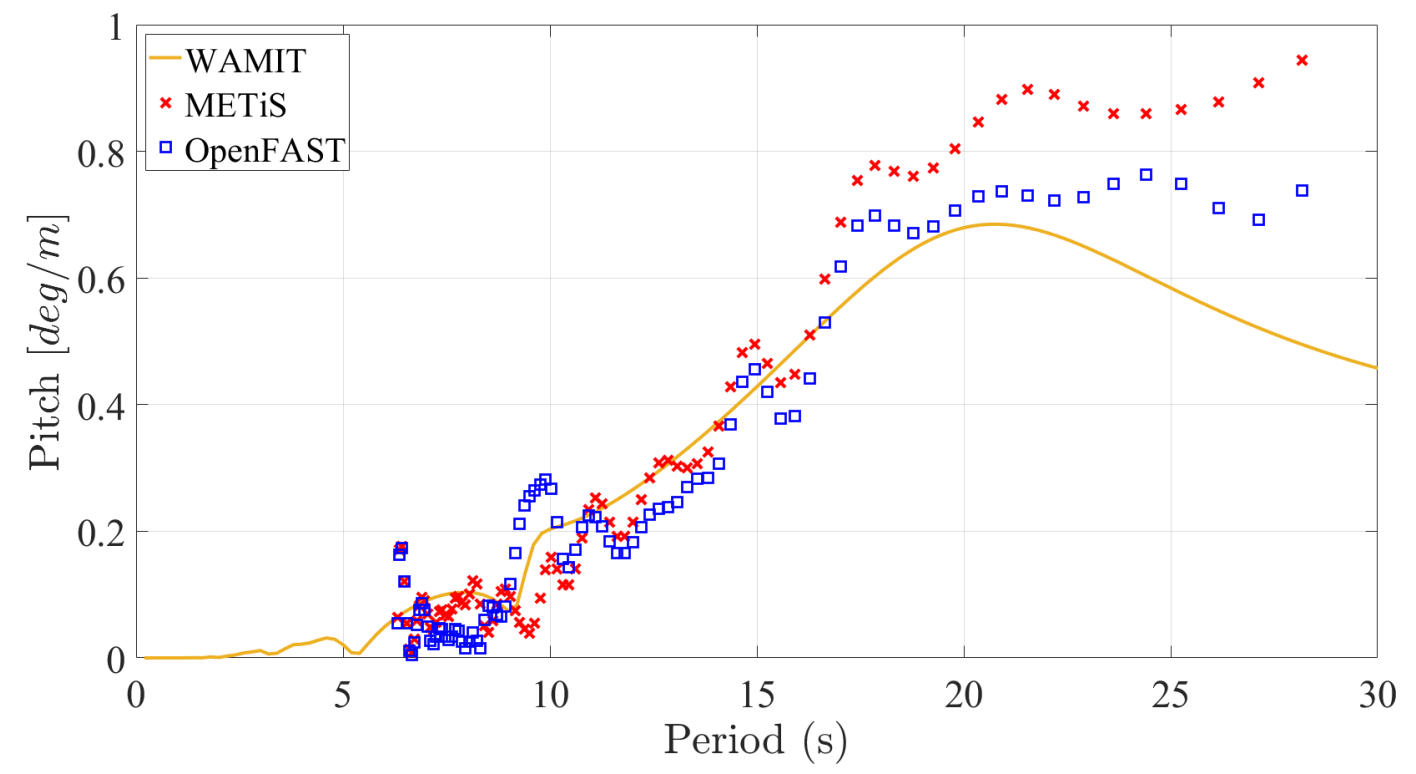

\subsection{Second-order surge force and motion under the action of bichromatic waves}

In order to analyze the second-order forces and motions, the first step is to verify the agreement of the first-order motions due to the bichromatic waves, as they affect the second-order results, which is done by comparing the first-order motion RAOs. For METiS and the experiments, the motions at the wave frequencies were obtained from the respective time series using the methodology presented in Section 5.2.1.2, which is also used ahead to evaluate the forces and motions at the difference-frequency.

The general agreement observed for surge and pitch was quite good, but since their impact on the second-order forces is small in the range of periods that was analyzed, these results are not shown here for conciseness. Heave, on the other hand, has a significant impact on the second-order forces, hence deserving a special attention. As shown in Figure 53, WAMIT matches quite well the experiment (except for the lowest period), while METiS provides good results except for the resonance peak, which is overestimated by almost $40 \%$. Given that the external linear damping in heave, $B_{33}^{\text {ext }}$, considered in METiS was extracted from WAMIT, it means that the axial quadratic drag, $C_{D z}$, that was tuned based on the forced oscillation tests did not perform well in the presence of waves. In order to assess the impact of this difference on the second-order surge force, a second set of METiS simulations was performed with an external linear damping equal to 
twice the value presented in Section 6.3.2. Though this is not strictly correct, since the problem is supposed to be the axial quadratic drag and not the external linear damping adopted in METiS, it is more practical to tune $B_{33}^{\text {ext }}$ because it is an unique value for all the simulations. As can be seen in Figure 53, this is enough to obtain a heave motion reasonably close to the experiment, which is what matters here.

Figure 53 - Heave RAO for a wave incidence of $180^{\circ}$ obtained with the bichromatic waves.

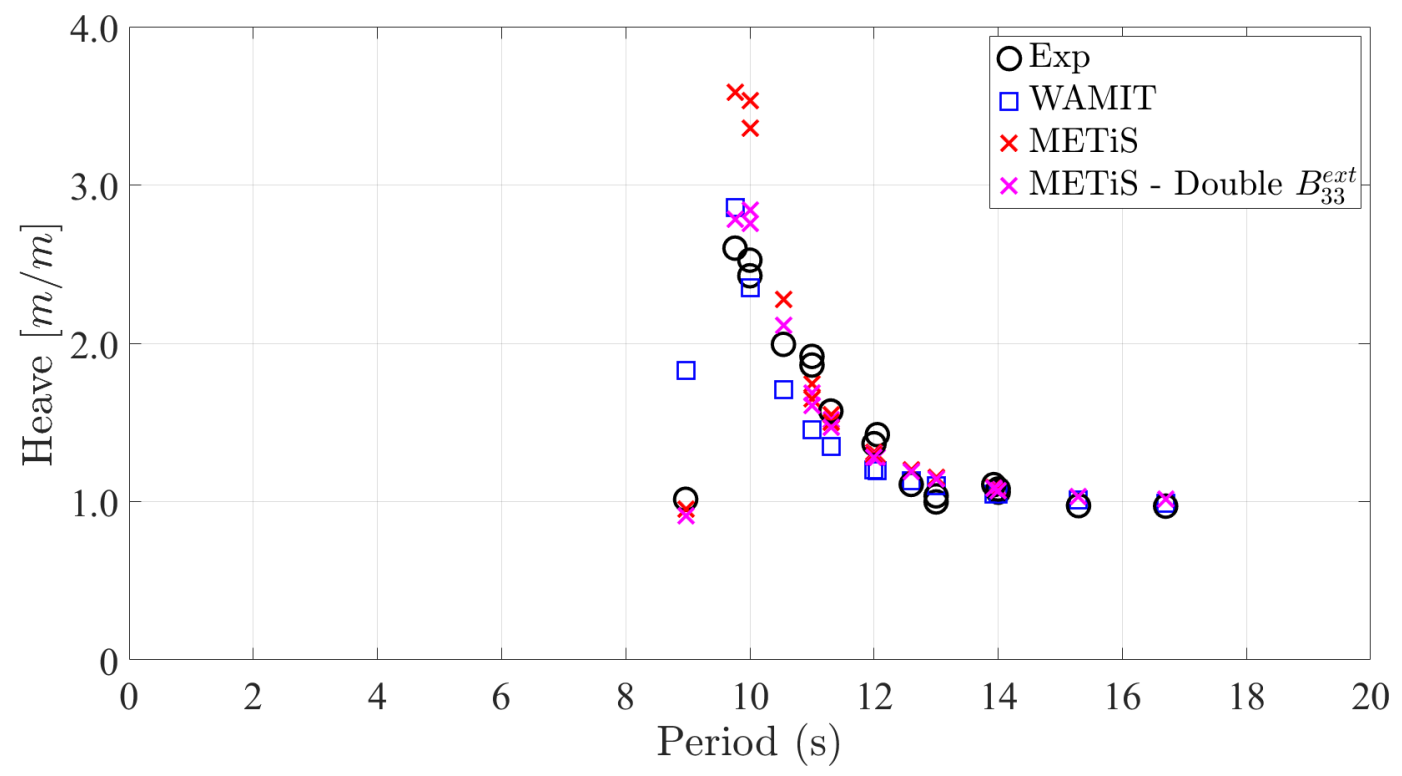

Figure 54 compares the second-order surge force obtained with WAMIT and METiS, considering the drag coefficients (METiS) and external linear damping (WAMIT) obtained from the forced oscillation tests and listed in Table 18. When body motions are not taken into account, the results agree within $30 \%$ for $T_{1}>11$ s, i.e. $\lambda_{1} / D_{\text {main }} \approx 12.6$ (it is worth reminding that $\lambda_{2}>\lambda_{1}$ ), with $D_{\text {main }}$ the diameter of the center column. This agreement is worse than the one observed for a single cylinder in Chapter 5, which showed a very good agreement for fixed cylinders under the action of bichromatic waves with $\lambda_{1} / D>10$. Here, however, the nondimensional drafts of the columns are lower $\left(\bar{d}_{\text {main }}=2.7\right.$ for the main column and $\bar{d}_{\text {side }}=4.4$ for the side columns), and it is possible that diffraction effects related to the interaction among columns are relevant.

Once the body is allowed to move, the forces significantly increase around the peak of first-order heave motion, as shown in Figure 54. The effect is larger in METiS, but the results predicted with both software get closer as the waves get longer. Bearing in mind the relative simplicity of the force model presented in this thesis, the agreement between METiS and WAMIT is quite good. As expected, increasing the external linear heave damping reduces the effect due to body motion around the resonance of heave.

Even though WAMIT predicts lower forces, it provides larger motions than the ones computed with METiS, as illustrated in Figure 55, meaning that the external damping factors calculated with Equation 6.10 are lower than the values that actually correspond 
Figure 54 - Nondimensional second-order surge force calculated with WAMIT and METiS.

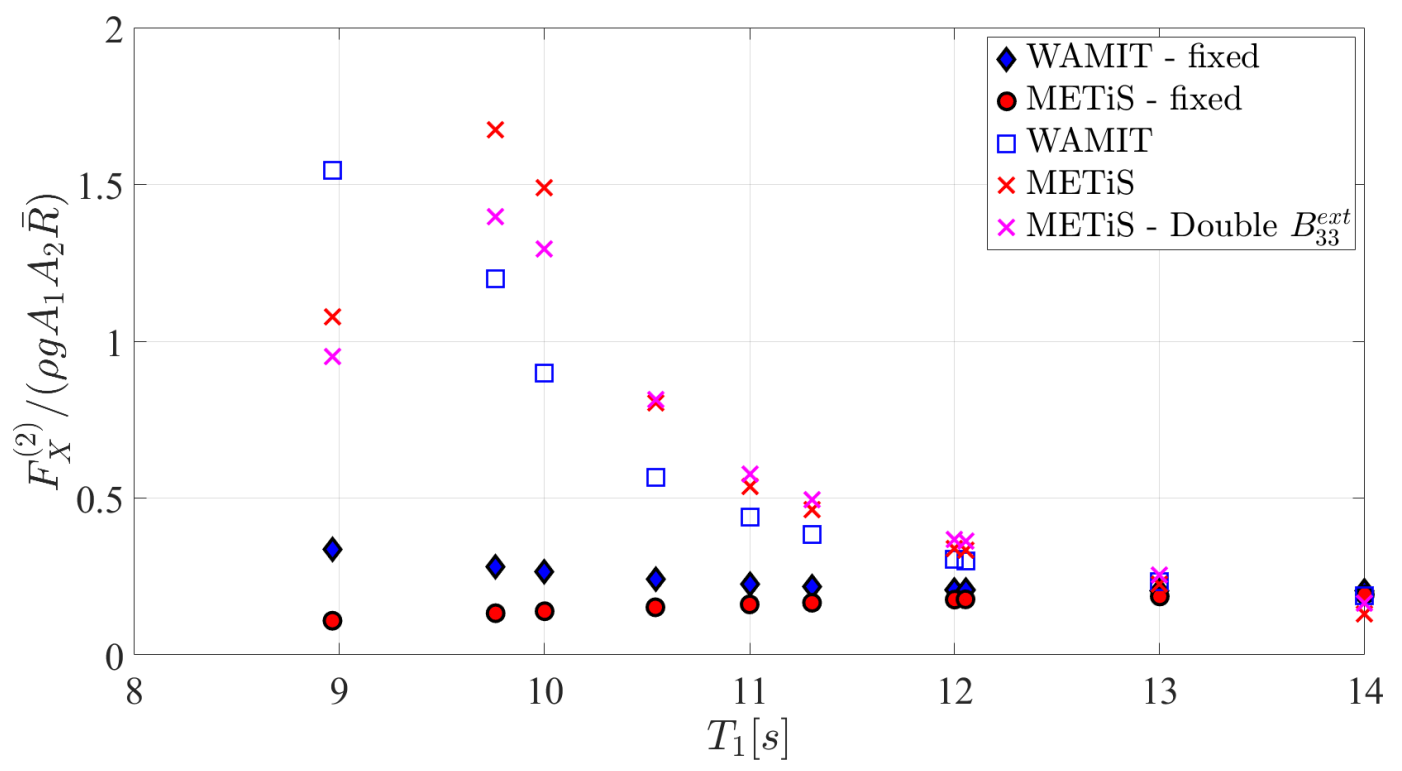

to the drag coefficients considered in METiS. One of the possible reasons is that this equation is valid for a purely sinusoidal motion, which was assumed to be at the frequency of the slow surge motion, while it is known that the first-order component also impacts the low-frequency damping when Morison's equation is used (MOLIN, 1993). Hence, the results show that the simple linearization approach given by Equation 6.10 does not hold for the analysis of the slow drift induced by bichromatic waves, and it should be modified to account for the first-order motion. If $B_{11}^{\text {ext }}$ is increased to about $2.5 \%$ of the critical damping in WAMIT, the results show a much better agreement, indicating that the required damping levels are indeed larger than the values that were evaluated.

Figure 55 - Difference-frequency second-order surge motion measured in the experiments and calculated with WAMIT and METiS.

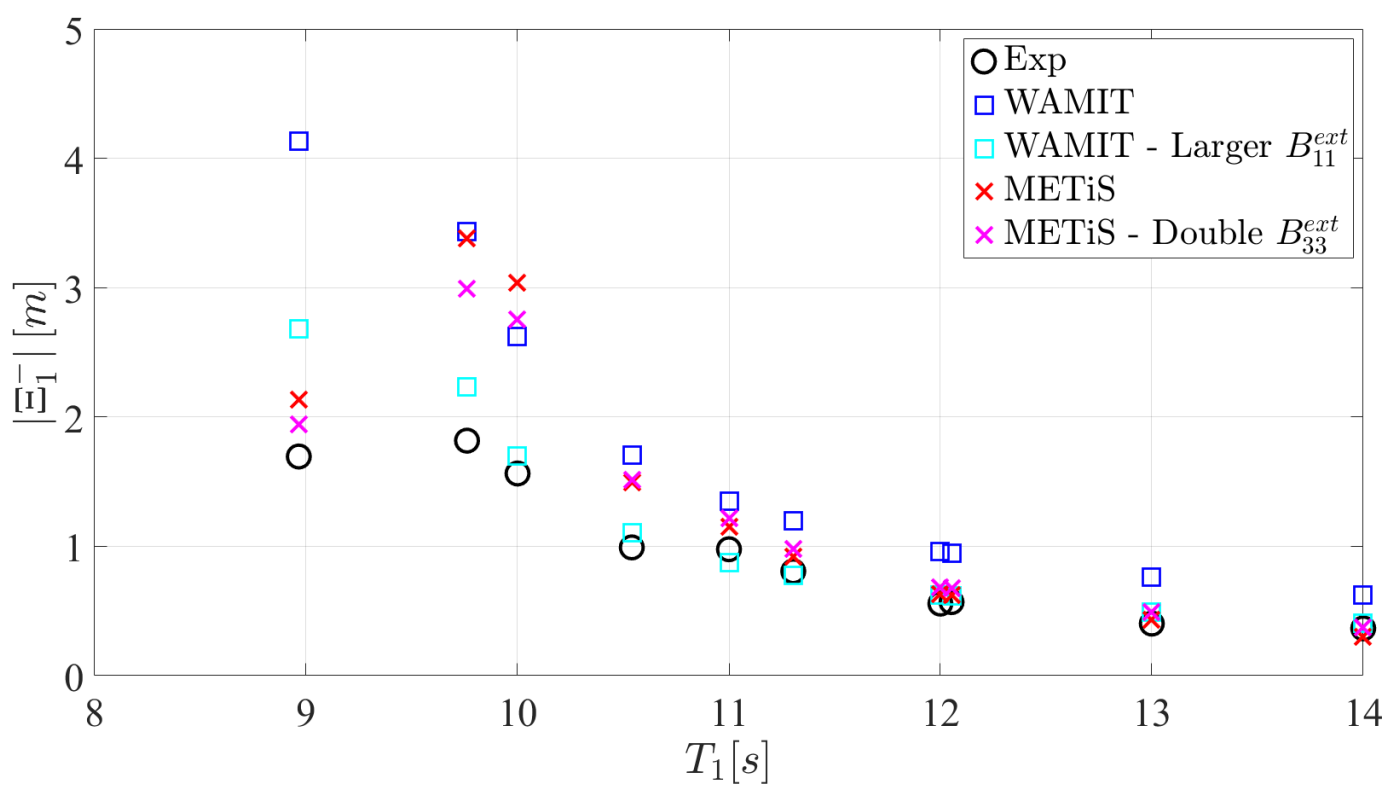


Meanwhile, the agreement between METiS and the experimental second-order surge motion is very good, except when the waves are within the heave resonance range, for which METiS predicts motions between 50\% and 90\% larger than the experiment. Although increasing the external heave damping in METiS improves the first-order heave and, consequently, reduces the discrepancy for these waves, it is not sufficient to match the experimental values. Since this does not occur for WAMIT with a larger $B_{11}^{\text {ext }}$, it is probable that this difference observed for METiS is, at least partially, due to radiation/diffraction effects, as expected from the values of $\lambda_{1} / D$ being close to the limit of the slender-body approximation for the second-order forces. Nevertheless, the good agreement observed for the longer waves attests not only the ability of the slender-body approximation to evaluate difference-frequency second-order loads, but also the utilization of the drag coefficients $C_{D x}$ obtained from the forced oscillations. In any case, it would be interesting to properly measure the uncertainties related to the determination of these drag coefficients, and then propagate them to the numerical results obtained considering these coefficients.

Besides damping, another important aspect that largely impacts the slow drift is the stiffness induced by the moorings. In the experiments, it was quite hard to measure the properties of the mooring lines, and even though a proper evaluation of experimental uncertainties was not conducted, they are expected to be significant. Due to the resonant nature of the slow drift and the small damping levels involved, a small change in the estimation of the natural period may lead to relatively large changes in the motions. Aiming at providing a general figure of the variations that may be expected on the motions, Figure 56 presents the results obtained with METiS considering a variation of $\pm 10 \%$ around the mean value of $\mathbb{K}_{M, G, 11}=6.92 \mathrm{e} 4 \mathrm{~N} / \mathrm{m}$, showing that it leads to variations between $20 \%$ and $30 \%$ on the slow surge motion, which emphasizes the importance of properly estimating the stiffness induced by the mooring system.

Figure 56 - Impact of mooring stiffness $\left(\mathbb{K}_{M, G, 11}\right)$ on the second-order surge motion.

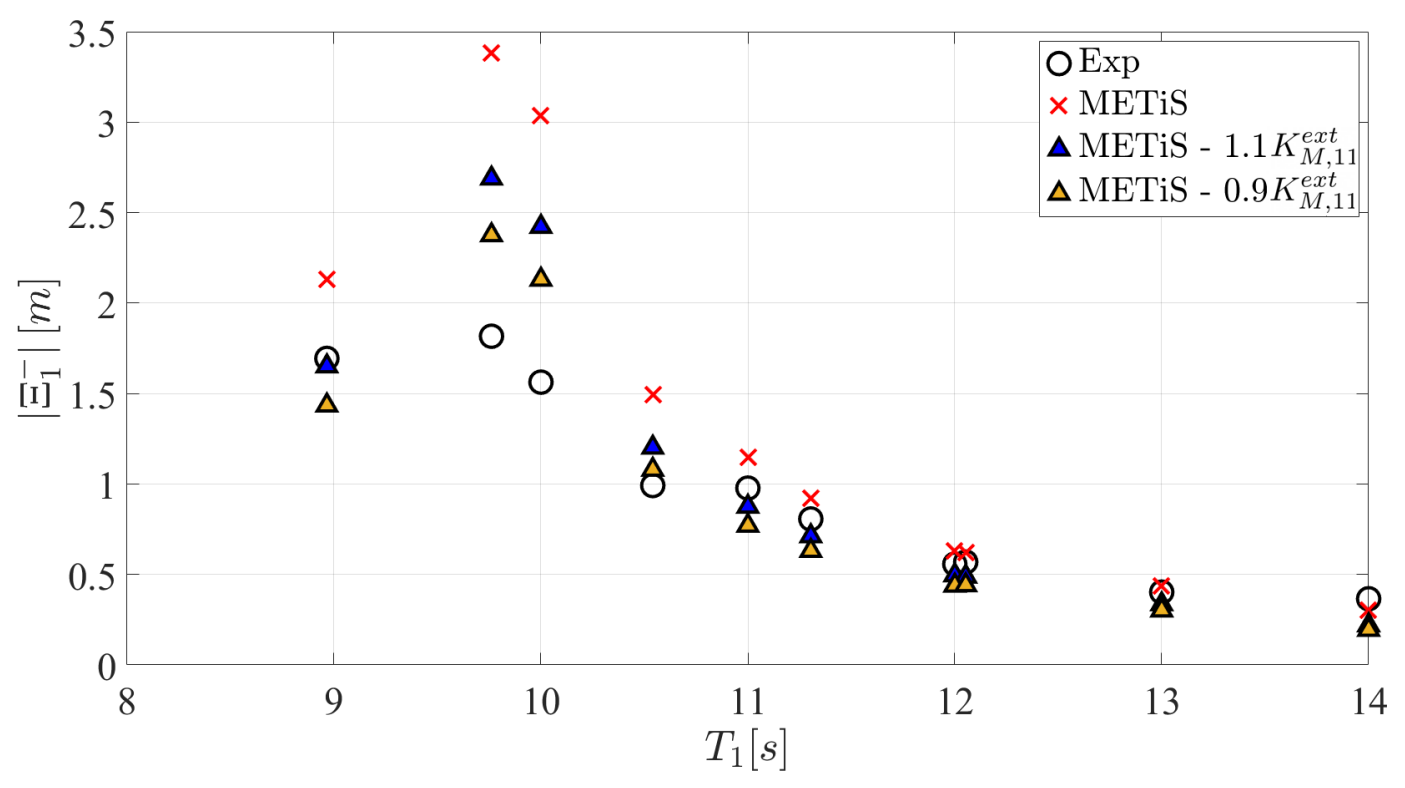


Given the usual difficulty in reproducing experimental slow drifts of FOWTs reported in the literature, the results obtained with both software are deemed more than adequate.

\subsection{Response under the action of irregular waves}

The irregular waves, whose characteristics are given in Table 15, were tested for a duration of $3 \mathrm{~h}$ in full scale. Just like in the analysis of the WHI01 wave, the records of wave elevation measured at the center of the tank during wave calibration were used as input to METiS and OpenFAST, thus guaranteeing that the waves analyzed in the numerical simulations were the same as the ones from the experiment. The results are time series of motion comprising components at a wide range of frequencies, as illustrated by the excerpt of experimental surge motion given in Figure 57, in which the slow-drift motion can be clearly distinguished from the motions at the frequencies of the waves. For this reason, the response of the floater is assessed by computing the power spectral density (PSD) of the time series of motions ${ }^{4}$. Like before, only surge, heave and pitch are analyzed, as these are the only DoFs with significant motion due to the wave incidence of $180^{\circ}$.

Figure 57 - Excerpt of the surge motion measured in the experiments for the JON01 wave.

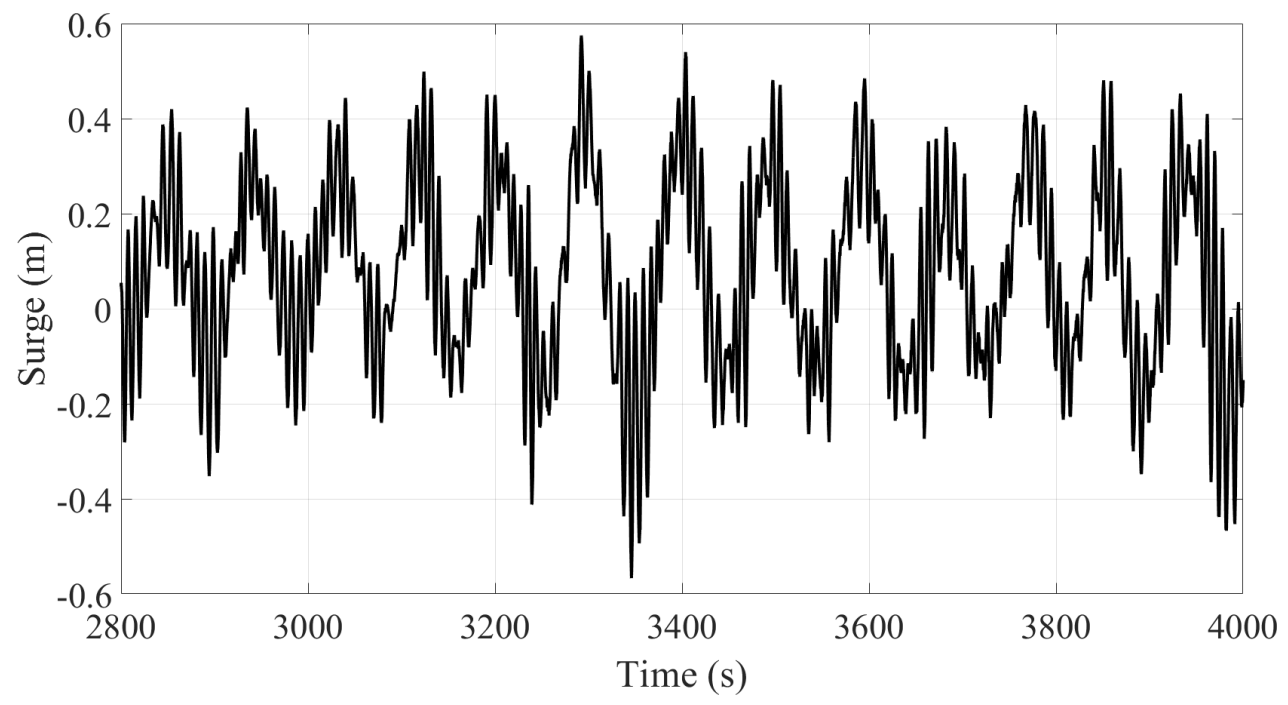

When the range of frequencies of the incoming waves does not encompass the natural frequency of interest, the motions of the structure due to second-order wave forces can be clearly distinguished from their first-order counterparts. In this case, it is useful to define a metric to compare the results obtained in each of these frequency ranges, and in this work the following is used:

$$
A_{\alpha}\left(\omega_{\text {low }}, \omega_{\text {high }}\right)=2 \sqrt{\int_{\omega_{\text {low }}}^{\omega_{\text {high }}} S_{\alpha}(\omega) \mathrm{d} \omega}
$$

\footnotetext{
${ }^{4}$ Using Welch's averaged, modified periodogram method with a Hamming window with $50 \%$ overlap.
} 
with $\omega_{\text {low }}$ and $\omega_{\text {high }}$ the frequencies that bound the range of interest. This expression is the same one used to compute significant amplitudes of wave elevation, an interpretation that is still valid when analyzing linear body motions. However, this is not strictly true when this formula is applied to the motions due to second-order forces, as the nonlinearity does not preserve the Gaussianity of the wave elevation. Nevertheless, it can still be used as a metric to compare the numerical responses with the ones measured in the experiments.

The ranges of interest are the one of the incoming waves, i.e. the frequencies with significant wave energy, and the ones around the surge and pitch natural frequencies (heave is not included because its natural frequency is inside the range of the incoming waves for all of the irregular waves analyzed). Due to the small mean drift observed for all of the wave conditions, the surge and pitch natural frequencies remain practically the same for all the sea states tested, as changes in the stiffness induced by the moorings are negligible. However, since different peak periods are analyzed, distinct $\omega_{\text {low }}$ and $\omega_{\text {high }}$ are required for each sea condition. These were established by inspecting the PSDs, and the resulting values are listed in Table 25. They are also identified in the plots by shading in light blue the area pertaining to the frequencies of the incoming waves and in light red the one around the natural frequency of the corresponding DoF. In some cases, these ranges overlap, but the amplitudes are calculated in the same way regardless of that.

Table 25 - Ranges of frequencies considered for computing the amplitudes around the surge and pitch resonances (red shaded in the graphs) and at the frequencies of the incoming waves (blue shaded).

\begin{tabular}{|c|c|c|c|}
\hline & & $\omega_{\text {low }}(\mathrm{rad} / \mathrm{s})$ & $\omega_{\text {high }}(\mathrm{rad} / \mathrm{s})$ \\
\hline \multirow{4}{*}{ 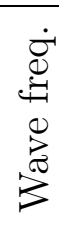 } & JON01 & 0.075 & 0.230 \\
\hline & JON02 & 0.050 & 0.225 \\
\hline & JON03 & 0.030 & 0.200 \\
\hline & JON04 & 0.030 & 0.200 \\
\hline \multirow{2}{*}{\multicolumn{2}{|c|}{ Surge resonance }} & 0.004 & 0.020 \\
\hline & & 0.033 & 0.058 \\
\hline
\end{tabular}

Starting with the JON02 wave $\left(T_{p}=12.0 \mathrm{~s}, H_{s}=2.0 \mathrm{~m}\right)$, which is the sea with the lowest peak period that is still within the expected range of validity of the slender-body approximation, the resulting motion spectra and amplitudes are given in Figure 58 and Table 26, respectively. To ease the comparisons between METiS and OpenFAST, the latter was run considering a linear mooring model. The results show that even though METiS somewhat overpredicts the vertical motions at the wave frequencies ( $8 \%$ in heave and $26 \%$ in pitch), the first-order motions are well reproduced. While the heave motion predicted with OpenFAST is also quite good, this is not the case for the pitch motion, which is about $50 \%$ larger than the experimental amplitude. The reason for this discrepancy between the first-order pitch motion predicted with METiS and OpenFAST is not clear yet, and they 
Figure 58 - Wave elevation and motion spectra for the JON02 wave.
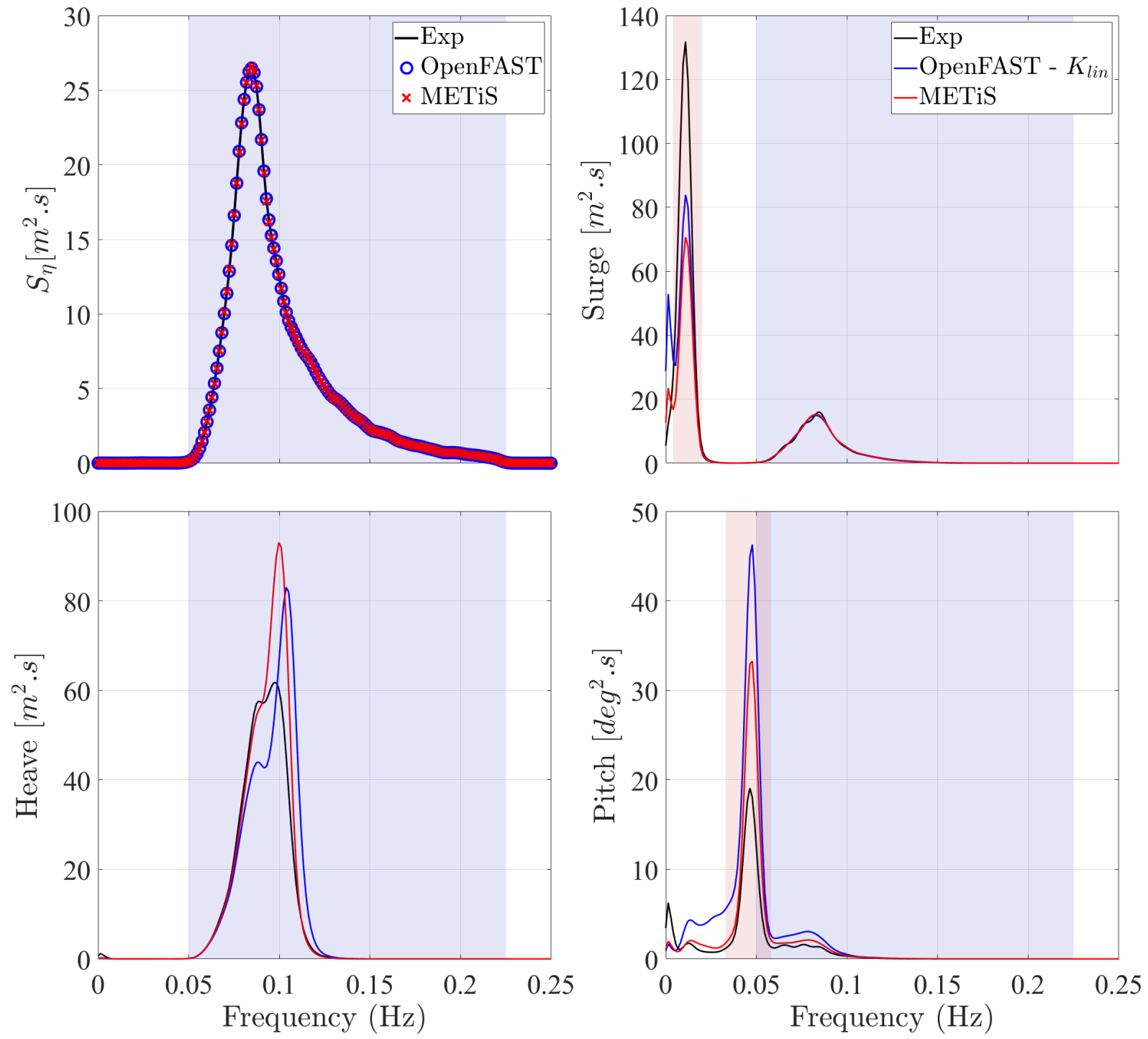

Table 26 - Motion amplitudes calculated with Equation 6.24 for the JON02 wave.

\begin{tabular}{lcccccc}
\hline & \multicolumn{3}{c}{ Wave frequency } & \multicolumn{3}{c}{ Natural frequency } \\
\hline & Exp. & OpenFAST & METiS & Exp. & OpenFAST & METiS \\
\hline Surge $(\mathrm{m})$ & 1.35 & 1.35 & 1.35 & 2.12 & 1.71 & 1.56 \\
Heave $(\mathrm{m})$ & 2.73 & 2.85 & 2.95 & - & - & - \\
Pitch $\left(^{\circ}\right)$ & 0.58 & 0.87 & 0.73 & 0.88 & 1.40 & 1.15 \\
\hline
\end{tabular}

cannot be due to the difference pointed out in Section 6.5.1 because in that case METiS provided larger pitch moments than the ones that were considered in OpenFAST, i.e. the opposite that is observed here.

Even though the slow surge amplitude obtained with both OpenFAST and METiS are about $75 \%$ of the one observed in the experiment, this difference can be attributed to the choice of drag coefficient, which, based on the forced oscillations, could be as low as $C_{D x}=0.50$ instead of the adopted value of 1.0. As already mentioned in Section 6.6, 
a measure of the uncertainties related to the drag coefficient would be very useful to properly compare the numerical results with the experiments. In this text, however, only a brief verification is performed by considering a simulation with different values of drag coefficients, which is presented at the end of this section. For now, it is enough to point out that the slow surge calculated with both software are very close, which is very important for the purposes of this thesis.

On the other hand, the numerical simulations overpredict the amplitude of slow pitch by about $30 \%$ for METiS and $60 \%$ for OpenFAST. When a dynamic mooring model is included in OpenFAST (a simulation whose results are not shown in the graphs for conciseness), this difference is reduced to $30 \%$, evidencing the relevance of drag due to the moorings to the resonant pitch motion. Then, it is reasonable to assume that the results obtained with METiS would be much better if a dynamic mooring model was used.

For the JON03 $\left(T_{p}=18.0 \mathrm{~s}, H_{s}=4.0 \mathrm{~m}\right)$ and JON04 $\left(T_{p}=18.0 \mathrm{~s}, H_{s}=8.0 \mathrm{~m}\right)$ waves, which are mostly composed of long waves, the results, given in Figures 59 and 60 and Tables 27 and 28, show that pitch motion is largely overestimated by both METiS and OpenFAST, indicating that the damping levels are underestimated. This also happens, with lower intensity, to heave around its natural frequency, but this is not reflected in the resulting amplitude because this discrepancy is diluted by the good agreement for the other frequencies. Part of this problem is due to the lack of a dynamic mooring model, but it is also due to the value of $C_{D z}$ adopted for the models, which was insufficient. Meanwhile, the first order surge is well captured.

However, this error on the first-order vertical motions is critical for METiS, as they are used to compute the second-order forces, leading to the large discrepancy observed for the slow surge motion. It is not so troublesome for OpenFAST, as what matters for this software is the first-order motions that were calculated in WAMIT when computing the force QTFs, in such a way that the second-order forces are independent from the results obtained in the time-domain simulation. Though in here this may seen an advantage, given that the results obtained with OpenFAST are better than the ones predicted by METiS, it is actually a drawback of the more complicated method adopted by the former, since the first-order motions that are observed in the time-domain simulations do not correspond to the ones that were actually considered in the evaluation of the second-order forces.

Given this known issue of properly choosing the drag coefficients, which is also faced by time domain tools based on radiation/diffraction, a valid question is whether the slender-body approximation is able to properly reproduce the experimental motions once these coefficients are well calibrated, provided, of course, that the wave length is long enough. The answer is affirmative, as illustrated by Figure 61 and Table 29, which compare the results measured in the experiments for the JON04 wave with the ones obtained with METiS considering $C_{D z}=3.0$ and $C_{D}=1.5$. They show that once there is a good 
Figure 59 - Wave elevation and motion spectra for the JON03 wave.
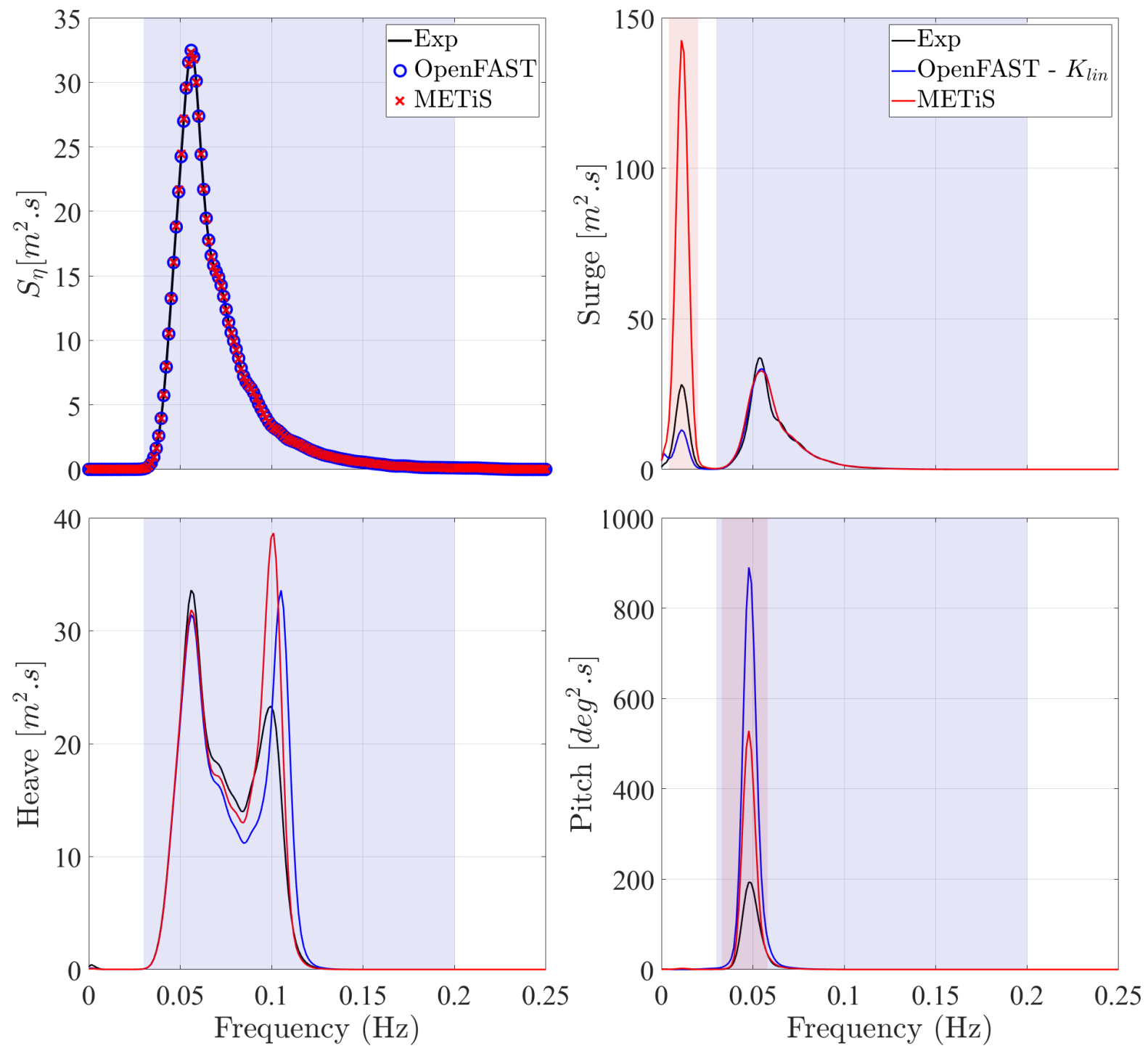

Table 27 - Motion amplitudes calculated with Equation 6.24 for the JON03 wave.

\begin{tabular}{lcccccc}
\hline & \multicolumn{3}{c}{ Wave frequency } & \multicolumn{3}{c}{ Natural frequency } \\
\hline & Exp. & OpenFAST & METiS & Exp. & OpenFAST & METiS \\
\hline Surge $(\mathrm{m})$ & 1.75 & 1.78 & 1.79 & 0.97 & 0.67 & 2.16 \\
Heave $(\mathrm{m})$ & 2.33 & 2.38 & 2.42 & - & - & - \\
Pitch $\left({ }^{\circ}\right)$ & 2.98 & 5.81 & 4.38 & 2.86 & 5.69 & 4.29 \\
\hline
\end{tabular}

agreement between the first-order motions, a good match for the second-order motions can be obtained with a drag coefficient that is within the range observed in the forced oscillations. Similar results are obtained for the JON02 and JON03 as well, though with different values of $C_{D}$, which, nonetheless, are still within the expected range. 
Figure 60 - Wave elevation and motion spectra for the JON04 wave.
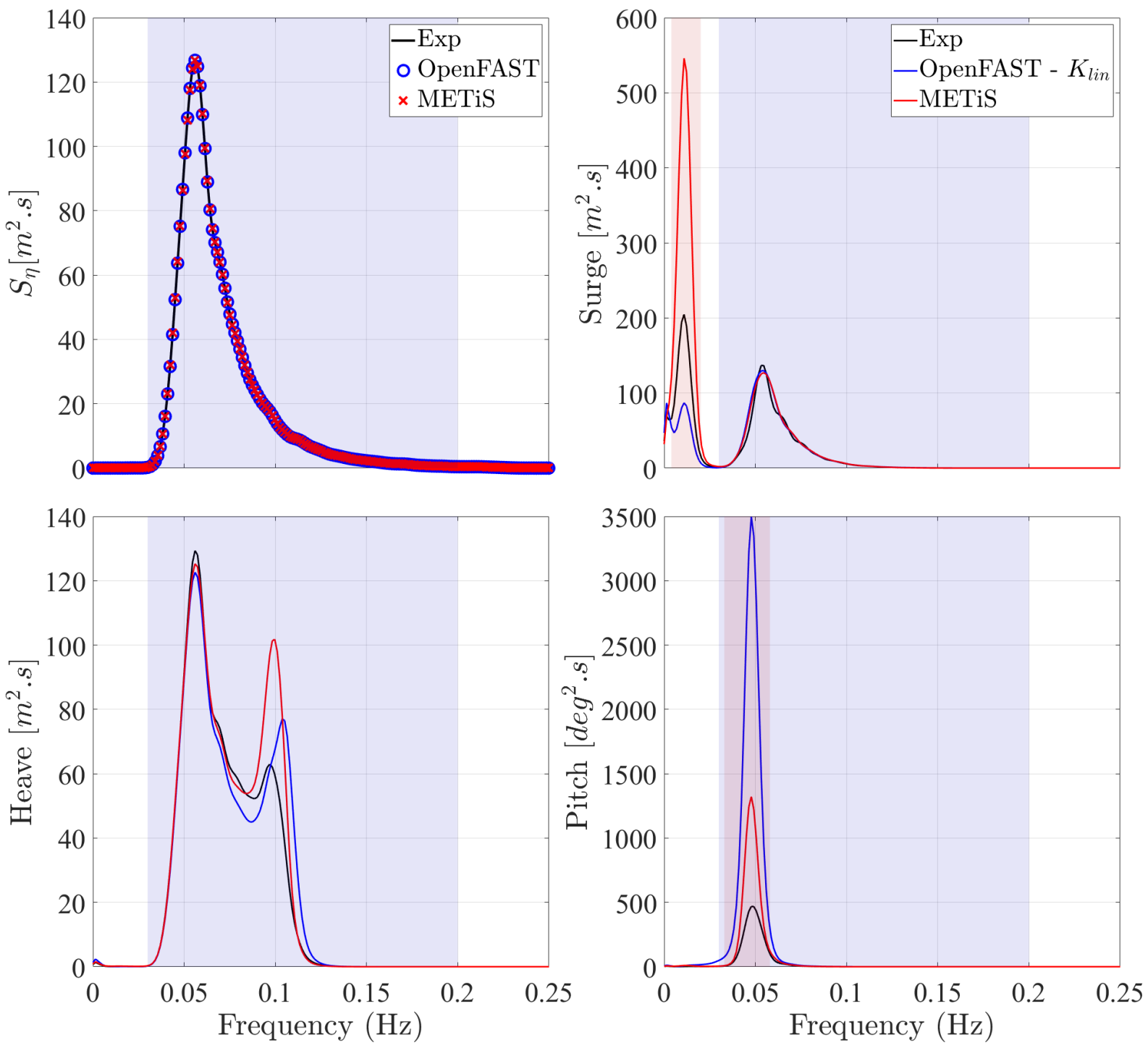

Table 28 - Motion amplitudes calculated with Equation 6.24 for the JON04 wave.

\begin{tabular}{lcccccc}
\hline & \multicolumn{3}{c}{ Wave frequency } & \multicolumn{3}{c}{ Natural frequency } \\
\hline & Exp. & OpenFAST & METiS & Exp. & OpenFAST & METiS \\
\hline Surge $(\mathrm{m})$ & 3.45 & 3.59 & 3.56 & 2.76 & 1.85 & 4.48 \\
Heave $(\mathrm{m})$ & 4.39 & 4.47 & 4.59 & - & - & - \\
Pitch $\left({ }^{\circ}\right)$ & 4.95 & 12.17 & 7.31 & 4.71 & 11.92 & 7.10 \\
\hline
\end{tabular}


Figure 61 - Wave elevation and motion spectra for the JON04 wave considering adjusted drag coefficients.
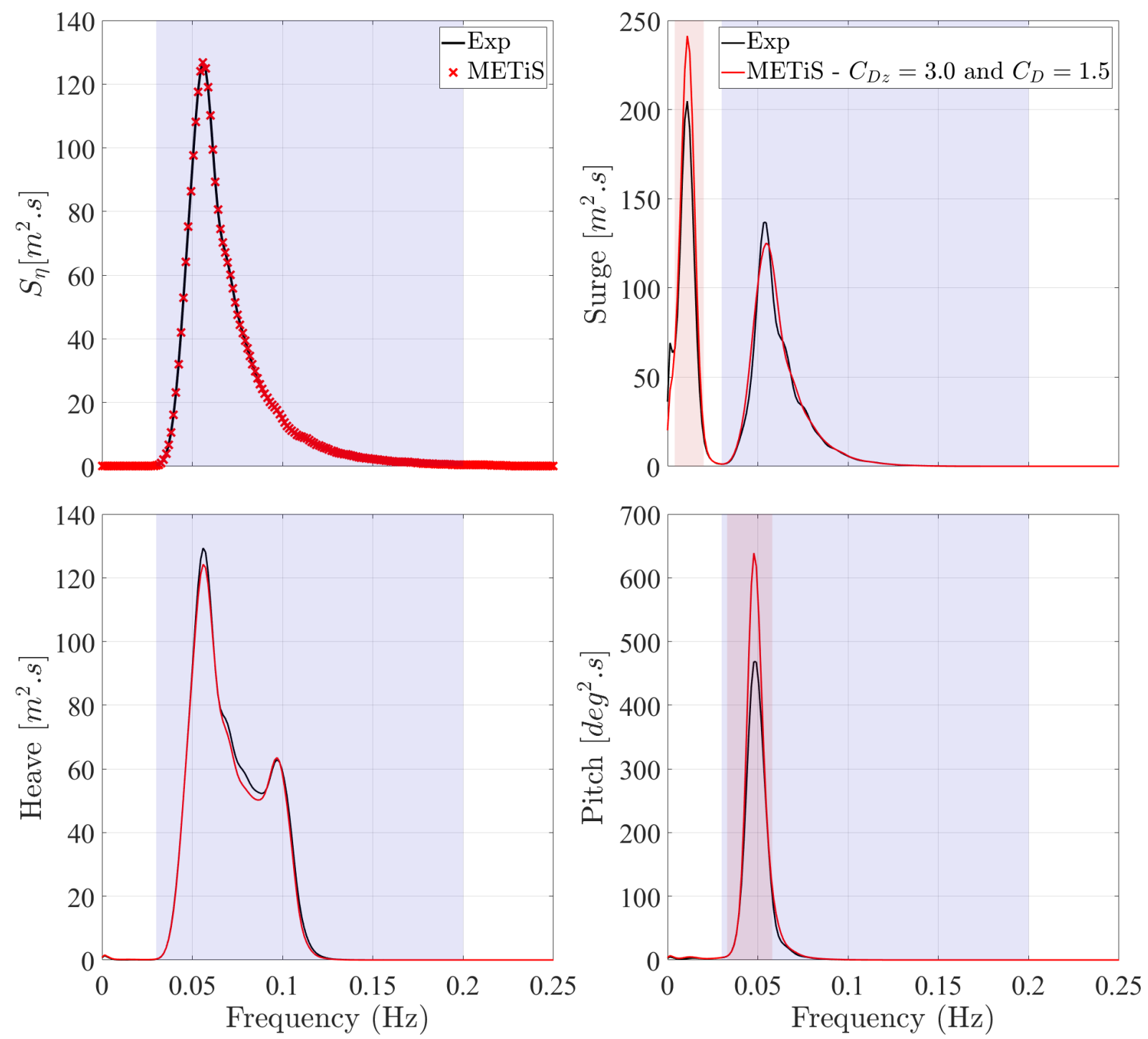

Table 29 - Motion amplitudes calculated with Equation 6.24 for the JON04 wave considering adjusted drag coefficients.

\begin{tabular}{lcccc}
\hline & \multicolumn{3}{c}{ Wave frequency } & \multicolumn{2}{c}{ Natural frequency } \\
\hline & Exp. & METiS & Exp. & METiS \\
\hline Surge $(\mathrm{m})$ & 3.45 & 3.53 & 2.76 & 3.03 \\
Heave $(\mathrm{m})$ & 4.39 & 4.32 & - & - \\
Pitch $\left(^{\circ}\right)$ & 4.95 & 5.49 & 4.71 & 5.23 \\
\hline
\end{tabular}

It is remarkable that the slow surge amplitudes obtained with METiS for the JON03 and JON04 considering the same coefficients (Tables 27 and 28) vary almost linearly with the wave significant height. Since the second-order force is proportional to the square of the wave height and the slow drift motion is a resonant phenomenon, this means that the damping must be proportional to the wave height. However, this is not the case for 
the experiment, and assuming that the second-order surge force for unitary $H_{S}, F$, is well captured by the numerical model, a reasonable hypothesis is that there is an extra damping factor in the experiments, $\gamma_{0}$, that is not considered in METiS. In other words, it is reasonable to assume that the motion amplitudes for METiS and the experiment can be described by the following relations ${ }^{5}$ :

$$
\begin{aligned}
& a_{\mathrm{mts}}=\frac{F H_{S}^{2}}{\gamma_{1} H_{S}} \\
& a_{\mathrm{exp}}=\frac{F H_{S}^{2}}{\gamma_{0}+\gamma_{1} H_{S}}
\end{aligned}
$$

and the idea here is to show that it is possible to predict the experimental response of the JON04 wave using the experimental and numerical amplitudes of the JON03 only. In fact, Equation 6.25 for the JON03 $\left(a_{\mathrm{mts}}=2.16 \mathrm{~m}\right.$ and $\left.H_{S}=4 \mathrm{~m}\right)$ yields $F / \gamma_{1}=0.54$, and this value in Equation 6.26 applied to the the same sea $\left(a_{\exp }=0.97 \mathrm{~m}\right)$ results in $\gamma_{0} / \gamma_{1}=4.91$. Substituting these values in Equation 6.26 provides the following amplitude for the JON04 wave:

$$
a_{\exp }=\frac{0.54 \cdot 8^{2}}{4.91+8}=2.68
$$

which is within $3 \%$ of the amplitude measured in the experiments. However, it is not possible to draw a conclusion based solely on the results for these two sea conditions, but, unfortunately, they were the only ones tested in the experimental campaign. As the evidence pointed out by the results above is quite interesting, a more systematic analysis encompassing different combinations of the parameters that describe the sea is intended in a continuation of this work.

Concerning the JON01, which is the sea with the lowest peak period $\left(T_{p}=8.0 \mathrm{~s}\right)$ and significant height $\left(H_{s}=1.0 \mathrm{~m}\right)$, METiS fails to model the second-order motions, as indicated by the results given in Figure 62 and Table 30. This was expected, as most of the wave components have a period below $11 \mathrm{~s}\left(\lambda / D_{\text {main }}<12.6\right)$, hence outside the range of validity of the slender-body approximation observed in Section 6.6. Nevertheless, the first order motions are well captured, evidencing that diffraction effects are more relevant to the second-order loads than for their first-order counterparts, which is reflected on the more rigid restriction on the ratio between the wave length and the diameter of the cylinders than the usual threshold $\lambda / D>5$ usually adopted for Morison's equation.

Finally, the motion amplitudes calculated from the simulations considering the wind condition specified in Sections 6.1.1 and 6.1.3 are given in Table 31 as a ratio with respect to the amplitudes computed without wind, while the spectra obtained for the JON02 are illustrated in Figure 63. Like already observed for the decay and WHI01 tests, heave motion is practically insensitive to the aerodynamic forces induced by the wind, and

\footnotetext{
${ }^{5}$ Thanks to Prof. Aranha for pointing out this simple model for predicting the response of the JON04 wave.
} 
Figure 62 - Wave elevation and motion spectra for the JON01 wave.
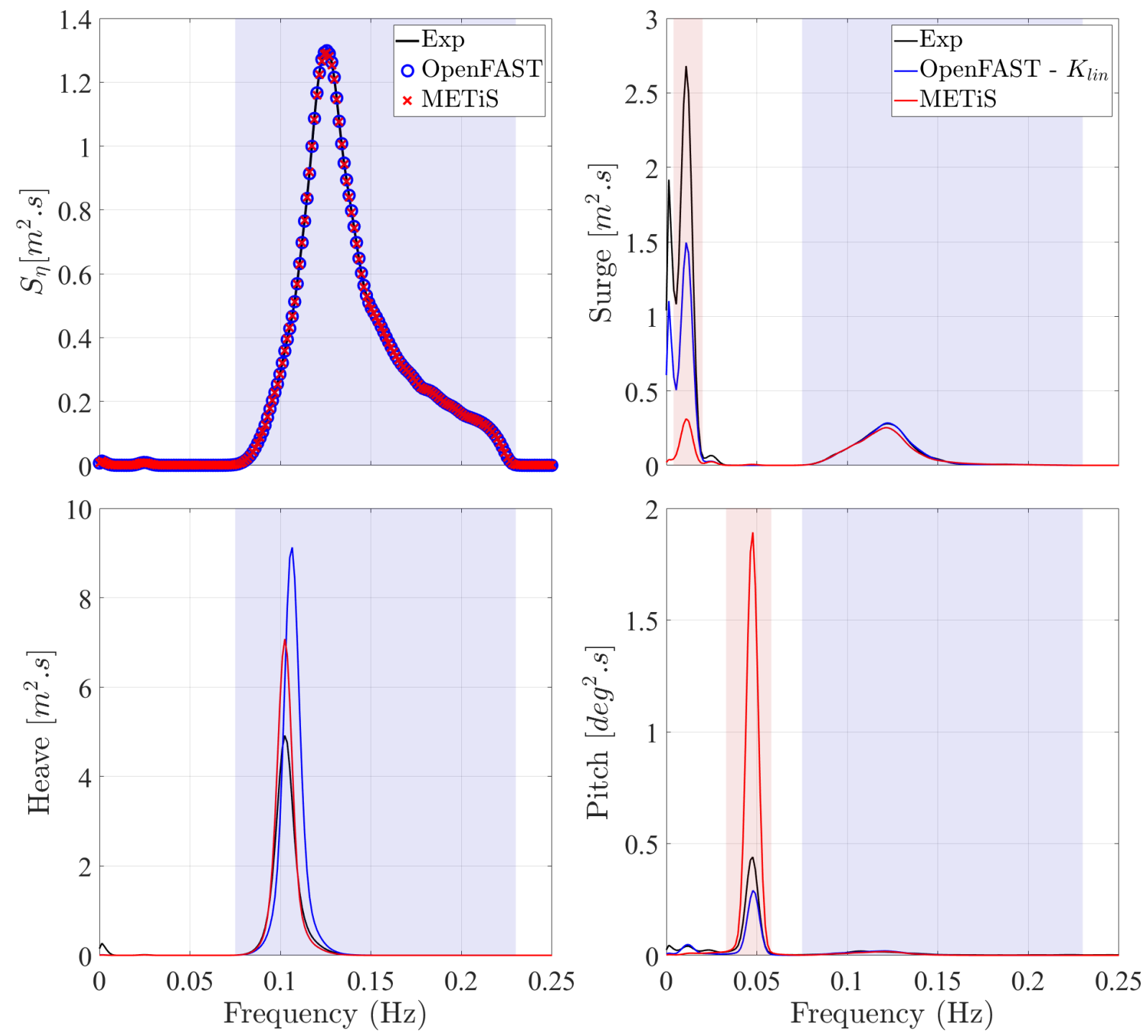

Table 30 - Motion amplitudes calculated with Equation 6.24 for the JON01 wave.

\begin{tabular}{lcccccc}
\hline & \multicolumn{3}{c}{ Wave frequency } & \multicolumn{3}{c}{ Natural frequency } \\
\hline & Exp. & OpenFAST & METiS & Exp. & OpenFAST & METiS \\
\hline Surge $(\mathrm{m})$ & 0.20 & 0.20 & 0.19 & 0.30 & 0.22 & 0.10 \\
Heave $(\mathrm{m})$ & 0.52 & 0.61 & 0.57 & - & - & - \\
Pitch $\left(^{\circ}\right)$ & 0.06 & 0.05 & 0.05 & 0.12 & 0.08 & 0.25 \\
\hline
\end{tabular}

the same is true for the first-order surge motion. However, the slow surge presents changes up to $17 \%$ when wind is included in the simulations, and even though the aerodynamic forces introduce an additional damping, this effect is overcome by the increase of the second-order surge force due to the inclination of the body and the reduction of mooring stiffness due to the mean displacement. The only exception is the JON01, for which the slow surge amplitude is reduced. In any case, both METiS and OpenFAST predict similar changes due to the wind. 
Table 31 - Ratio between motion amplitudes with and without wind $\left(A_{\alpha}^{\text {wind }} / A_{\alpha}\right)$.

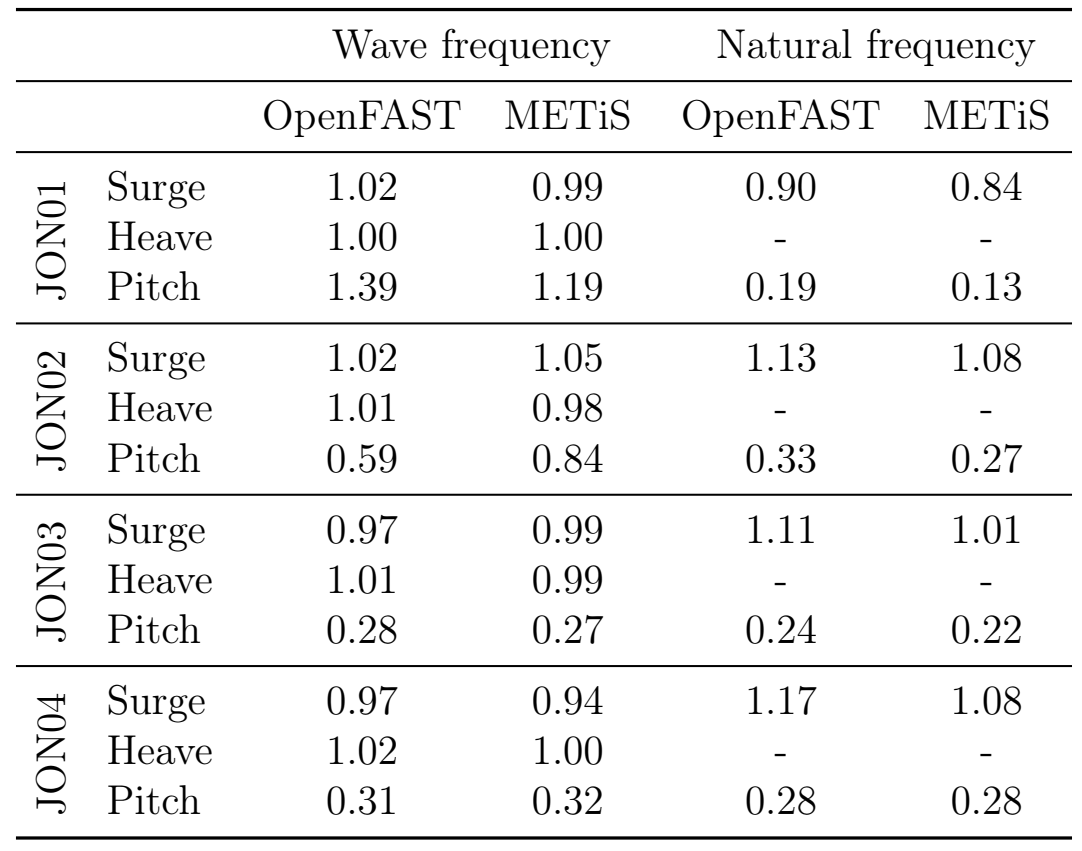

Figure 63 - Wave elevation and motion spectra for the JON02 with and without wind.
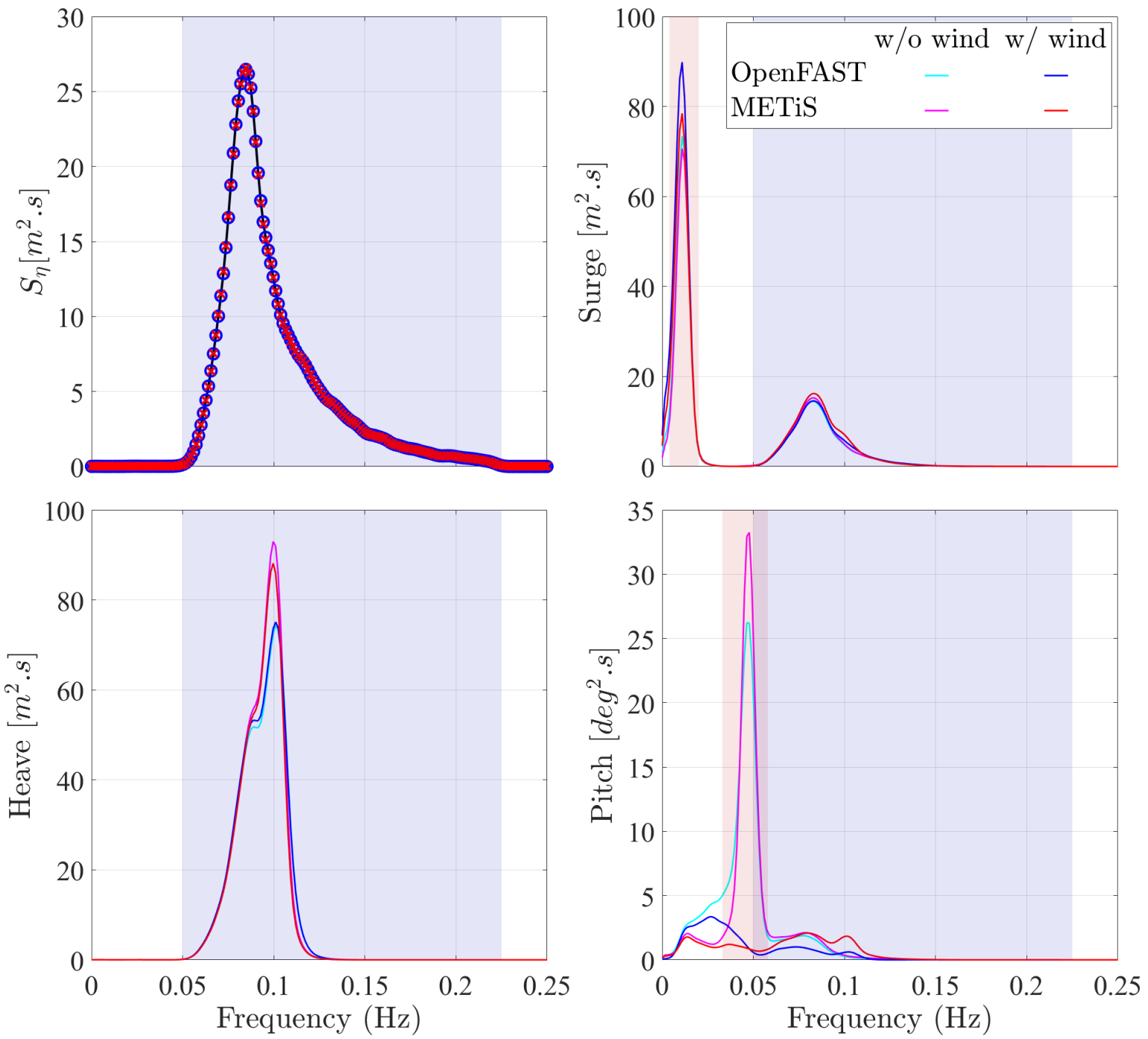
Pitch is the DoF that is impacted the most by the wind, and METiS and OpenFAST predict very similar amplitude changes. Nevertheless, they show significant discrepancies when looking at the spectrum, as exemplified by the JON01 given in Figure 64. Looking at the region with wave energy, the OpenFAST result with wind presents a pronounced peak that is due to coupling with heave, more specifically due to the $A_{35}$ term of the added mass matrix, which is significantly lower in METiS. This is due to the fact that the $A_{35}$ calculated with METiS is half the value computed with WAMIT (for $\omega=0$ ), which is a consequence of taking the added mass coefficients, $C_{a}$ and $C_{a z}$, as being the same for all the columns. This error also occurs for $A_{55}$, but this was not a problem up to now because it is only about $10 \%$ of the corresponding moment of inertia of the body, $I_{y y}$, but it is clear that a more careful choice of added mass coefficients should be followed when either $A_{55}$ or $A_{35}$ are relevant. This larger peak in OpenFAST is compensated by an almost complete absence of motion around $f=0.12 \mathrm{~Hz}$, which is not observed in METiS, in such a way that even though the results presented in Table 31 are very similar, the corresponding spectra of pitch motion in this case are actually quite different.

Figure 64 - Spectrum of pitch motion for the JON01 wave with and without wind, zoomed to show the response at the wave frequency.

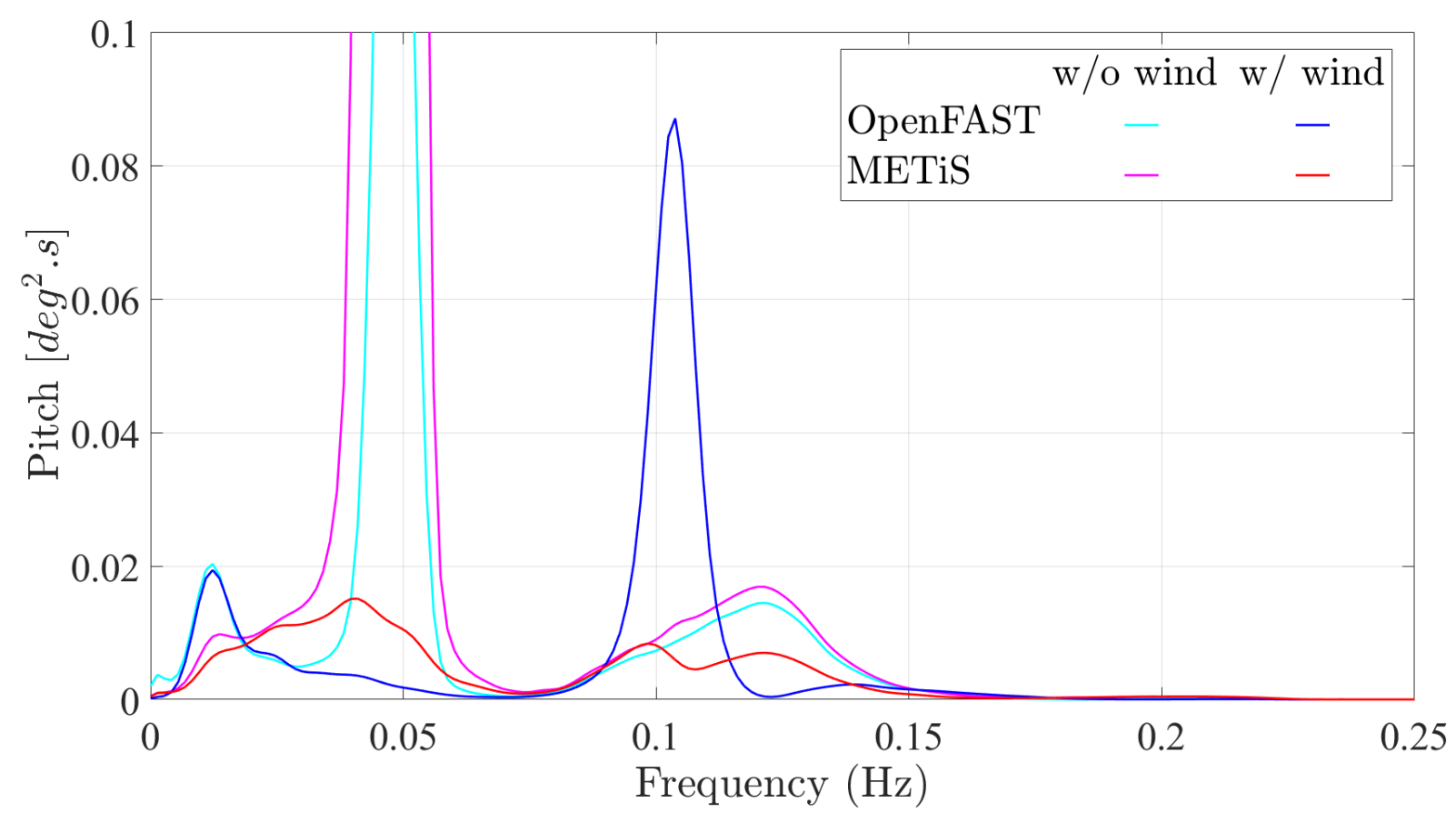

Since the main objective of the slender-body approximation is to save time, the simulation time spent on the irregular waves is quite relevant. The three-hour METiS simulations without wind took between 8 and 9 minutes, while the ones with wind took 15 minutes at most. Meanwhile, the OpenFAST simulations without wind and considering a linear mooring system took about 55 minutes, which is increased to 90 minutes once wind is included and further to roughly 120 minutes if both aerodynamics and mooring dynamics are solved (all the simulations were performed with the same computer with 
an Intel ${ }^{\circledR}$ Core $^{\mathrm{TM}} \mathrm{i} 7-37703.40 \mathrm{GHz}$ ). Bearing in mind that this is only the time spent to run the simulations in OpenFAST, hence it is not taking into account neither the 11 hours needed to compute the QTFs with WAMIT nor the time spent building the mesh, it becomes clear that the slender-body approach is an interesting way of computing the second-order motions of a FOWT, at least for early stages of the design.

Nonetheless, as shown by the results presented in this chapter, one should be aware of the limitations of the slender-body approximation to the analysis of secondorder motions, namely seas that correspond to $\lambda / D$ larger than a threshold that, based on Chapter 5, depends on the nondimensional water depth, nondimensional differencefrequency of interest and aspect ratio of the cylinders, but which seems to be at least 10 . Moreover, the major difficulty with this approximation lies in the proper choice of the added mass and drag coefficients, specially the latter, which should then be determined carefully and taking into account the associated uncertainties. This, however, is also true for simulations based on radiation/diffraction, since these coefficients directly impact damping levels and, consequently, the slow-drift amplitudes.

Hopefully, the analyses presented in this chapter have also demonstrated how complex the dynamics of a FOWT is, with important couplings arising from inertial, hydrodynamic, aerodynamic and mooring forces. Therefore, the development of numerical tools that are capable of modeling all these effects is essential not only to design these structures, but also to provide a better understanding of their dynamics. 



\section{Conclusion}

This thesis presented a slender-body approach for evaluating the first- and second-order wave loads acting on a structure comprised of slender cylinders, aiming at modeling both the wave frequency and slow-drift motions of floating offshore wind turbines (FOWTs). This approach was obtained by combining the formulation proposed by Pinkster (1980) for the second-order wave forces on floating bodies with the slender-body approximation for the nonlinear loads on cylinders independently obtained by Madsen (1986), Rainey (1989), and Manners (1992). This was done in such a way that their original slender-body approach, which evaluates the forces considering the instantaneous position of the cylinder, was modified to compute the wave loads considering the mean body position. Though this difference may seen subtle, it results in a huge reduction of computational cost for the simulation of real sea conditions because it allows to replace the simple summation of the contribution due to each wave component by an IFFT algorithm to compute the time series of wave kinematics and second-order wave loads. The force model is complemented with the quadratic drag term from Morison's equation and additional axial forces that are similar to the ones usually included when modeling floating structures with this equation. The resulting approach is, then, quite handy because the wave loads, up to second order, can be evaluated directly in time domain concomitantly with the other forces acting on the body, in opposition to the common approach of using hydrodynamic coefficients that need to be pre-computed in frequency domain.

This, however, comes with a cost. While Pinkster's formulation, which is the one followed by most radiation/diffraction codes, requires the wave steepness and body motions to be small, Rainey's formulation neglects wave radiation and scattering effects, besides modeling end effects due to the extremities of the cylinder in a very simplified way. Since the former is already adopted by the majority of the modern software used for the seakeeping analysis of FOWTs, the approach proposed in this thesis introduces the restrictions of the latter, which are acceptable as long as the diameter of the cylinders that compose the structure are small compared to their draft and to the incoming wave length. Moreover, structures that are not comprised of cylinders cannot, of course, be analyzed with this method. Though these conditions may be too restrictive for modern O\&G spars and semi-submersibles, which have large diameter columns, it is satisfied by most FOWT concepts in many significant wave conditions.

The implementation of this method led to the development of METiS-USP (Morison Equation Time Domain Simulation - University of São Paulo), a numerical tool for the seakeeping analysis of floating offshore wind turbines. At present, METiS is capable of evaluating the aerodynamic loads on the rotor with Blade Element Momentum Theory 
and the first- and second-order wave loads on the floater using the slender-body approach presented in this thesis. Since the computation of the second-order wave forces requires the first-order motions to be obtained in advance, at each time step the equations of motion are first solved considering only the linear terms (first-order problem) and, then, the results are used to compute the total forces acting on the body and the corresponding total motions. In order to numerically integrate the equations of motion, a standard $4^{\text {th }}$ order Runge-Kutta method with fixed step was adopted. The development of METiS is intended to be continued in the future with the contribution of other members of the research group, who have been studying other disciplines that are important to the coupled dynamics of a FOWT, such as rotor dynamics and control, elasticity and mooring dynamics. The goal is not only to have a new tool available for the analysis of such devices (especially considering that there are other codes - even open-source ones - that do this job in a very complete manner), but mainly to count with an in-house development upon which the performance of alternative approaches, mathematical models or solving algorithms can be tested.

In order to verify the slender-body approach and establish the conditions in which it is acceptable, the simple case of a single surface piercing cylinder under the action of long-crested bichromatic waves was analyzed for different combinations of the dimensionless parameters that describe the problem. First, the cylinder was taken as bottom mounted, which is a classic hydrodynamic problem with analytical solution for both diffraction theory (KIM; YUE, 1990) and the slender-body approximation. By separating the loads due to the undisturbed incident waves from the flow perturbation introduced by the body, it was shown that the complex amplitudes of the former are purely imaginary numbers, while the complex amplitudes of the latter have a real part that is associated with diffraction effects, which are increasingly relevant as the ratio of wave length to the diameter of the cylinder, $\lambda / D$, gets smaller. It is remarkable that the slender-body approximation, which provides purely imaginary complex amplitudes for all the force components, models very well the imaginary part of the loads even for the shortest waves that were analyzed (i.e. $\lambda / D=0.5$ ), evidencing that the problem with the slender-body approximation comes, indeed, from the approximated treatment of the diffraction potential that neglects effects such as wave scattering. Though it is difficult to point out a threshold in terms of $\lambda / D$ above which these effects are negligible, as the relative relevance of the different force components, notably the one due to the second-order potential, are dependent on the other parameters that characterize the problem, it seems that $\lambda / D>10$ is a good general value for the validity of the slender-body approximation. This is the same threshold indicated by Rainey (1995) for the validity of his formulation.

On the other hand, Newman's approximation, which is based on approximating the difference-frequency loads from the mean loads, is only capable of modeling the real part of the force, hence only the part of the force that is due to diffraction effects. Besides 
being fundamentally interesting for highlighting the physical differences between these two approximations, this observation is quite useful in practical terms, for it indicates that the different approaches (namely, Newman's and the slender-body approximations) can possibly be combined to obtain the total difference-frequency second-order force without accounting for the same effects twice.

The complexity of the test case was then increased, and two different floating cylinders were analyzed in order to assess the impact of the first-order motions on the second-order loads. Since this case does not have an analytical solution, the solution with the slender-body approximation was obtained with METiS, while WAMIT was used to solve the problem with radiation/diffraction theory. A good general agreement was observed for the horizontal force for $\lambda / D>10$, which is the same threshold observed for the bottom mounted cylinder, but the agreement was not as good for the second-order moment (given with respect to the mean water line). The reason for this is that end effects, both related to the incoming flow and to the first-order pitch motion, are more relevant for the moment than for the force, as they are magnified by the lever arm.

However, in opposition to the fixed case, the second-order force calculated with METiS has a non-zero real part, in such a way that the complementarity between Newman's and the slender-body approximation is not as easy to be exploited (some suggestions of possible alternatives are given ahead in Section 7.1). Furthermore, as both the vertical acceleration and the pressure have a $90^{\circ}$ phase with respect to the horizontal acceleration, it follows that the vertical force predicted with the slender-body approximation has a real amplitude even when the body is not allowed to move, in such a way that there is no complementarity with Newman's approximation for the vertical forces.

For both of the test cases discussed above, it is noteworthy the relevance of the force terms from Rainey's formulation that are usually not included in analyzes that extend Morison's equation to model second-order wave loads. In the discussion concerning the bottom mounted cylinder, it was shown that both the convective and axial-divergence accelerations need to be considered in order to match the force arising from the pressure drop due to the velocity squared (force component II from Pinkster (1980)), while the relevance of the rotation force term from Rainey's formulation was evidenced in the analysis of the floating cylinders. Therefore, it was shown that the common practice of analyzing second-order loads by simply applying Morison's equation with second-order wave kinematics is not strictly correct, since these additional terms from Rainey's formulation should not be disregarded before verifying their impact on the results.

The method was then applied to the analysis of the JPK FOWT, a semi-submersible FOWT model, moored by three caternary lines, that was tested at the wave basin of the Numerical Offshore Tank of the University of São Paulo. Three sets of tests were presented: forced oscillations of the hull, which were used to assess the added mass and 
drag coefficients to be considered in the numerical models; free decays of the moored model; and motions under the action of waves (bichromatic, JONSWAP and white-noise) and wind. The results obtained with METiS were compared with the experiments and with the ones computed with WAMIT and OpenFAST.

Several conclusions were drawn from the decay tests, which were analyzed using the classic PQ method, but the most remarkable ones are that the numerical tools could predict very well the oscillation periods measured in the experiments; that viscous effects seem to impact not only the quadratic part of the damping, but also its linear part, as the values obtained from wave radiation were not enough; that drag due to the moorings has a significant impact on heave and pitch motion, but not in surge; and that the wind almost completely dampens the pitch motion. Moreover, the changes introduced by the wind were very similar in both software, which was a useful step of validation for the aerodynamic calculations considering body motions.

Then, the first-order wave forces obtained with METiS were compared with the ones computed with WAMIT. The loads in the six degrees of freedom showed a good agreement for waves with period above $6 \mathrm{~s}$, which is about the threshold of $\lambda / D>5$ usually adopted for Morison's equation when taking the mean diameter of the columns, $\bar{D}=10.5$, as the characteristic length. The first-order motion RAOs, obtained from the experiments, METiS and OpenFAST via the response of the floater under the action of a white-noise wave, also matched quite well. For both forces and motions, the DoF with the worst agreement for METiS was, once again, pitch, and the reason is the same that was already pointed out for the floating cylinder. Nevertheless, this is acceptable, since METiS is supposed to be a fast and simple tool for early design stages. When wind was included, the peak of pitch motion was completely flattened, with the different software providing similar results.

The second-order surge force was then verified for the JPK FOWT under the action of bichromatic waves. When body motions were not taken into account, the results obtained with METiS were within $30 \%$ of the ones calculated with WAMIT for $\lambda_{1} / D_{\text {main }} \approx 12.6$ (with $D_{\text {main }}$ the diameter of the center column), an agreement that is worse than the one previously observed for a single cylinder. At present, it is still unknown exactly why this happens, but it is worth pointing out that the nondimensional drafts of the columns of the JPK are lower than the ones of the floating cylinders analyzed in this text, and it is possible that diffraction effects related to the interaction between columns are relevant. Once the body was allowed to move, the forces significantly increased around the peak of first-order heave motion. This effect was larger in METiS, but the results predicted with both software got closer as the waves got longer. Bearing in mind the relative simplicity of the force model presented in this thesis, the agreement between METiS and WAMIT can be considered quite good. 
The motions induced by the second-order surge force were also compared with the ones measured in the experiment. In order to damp the motions, a set of drag coefficients was established based on the amplitudes of slow-drift motion measured in the experiments and the results obtained from the forced oscillation tests, yielding different values of drag coefficients, ranging between $C_{D x}=0.66$ and $C_{D x}=2.45$, for each wave condition simulated in METiS. Though three out of the ten bichromatic waves showed significant discrepancies, which were attributed, at least partially, to radiation/diffraction effects, the general agreement observed between METiS and the experiment was remarkably good. This attested not only the utilization of the slender-body approximation to evaluate difference-frequency second-order loads, but also the drag coefficients $C_{D x}$ obtained from the forced oscillations.

For the WAMIT analyzes, the drag coefficients were linearized (based, again, on the amplitudes of slow-drift motion measured in the experiments) to provide a linear external damping, $B_{11}^{\text {ext }}$, to partly account for viscous effects. Even though this procedure results in a reasonable estimation of the second-order surge motion, it was concluded that these values of $B_{11}^{\text {ext }}$ underestimated the damping levels that actually correspond to the drag coefficients considered in METiS. By increasing $B_{11}^{\text {ext }}$, the results showed a much better agreement with the experiments, indicating that the required damping levels were indeed larger than the ones evaluated with the linearization procedure. The importance of properly estimating the stiffness induced by the mooring system was also emphasized by showing results with different stiffness values.

Finally, the JPK FOWT was analyzed under the action of four distinct JONSWAP waves. As expected, METiS performed poorly for the sea condition mostly composed of short waves $\left(T_{p}=8.0 \mathrm{~s}\right)$, but the performance was quite satisfactory for the wave conditions comprised of longer waves $\left(T_{p}=12.0 \mathrm{~s}\right.$ and $\left.T_{p}=18.0 \mathrm{~s}\right)$. The modeling difficulties that were faced were not related to the slender-body approximation itself, but were rather due to the lack of a dynamic mooring model and the difficulty of establishing the drag coefficients, a problem that is faced by other engineering tools that are currently employed in the design of FOWTs. It is also worth mentioning that the JPK FOWT was only considered in this text because it was the one with experimental results available, and there are other structures, such as spars or even other concepts of semi-submersibles, that would be more suitable to be analyzed with the slender-body approximation for being comprised of cylinders with smaller diameters.

A very important aspect is that METiS simulations were significantly faster than the ones using OpenFAST, besides being far simpler to run for not requiring pre-computed hydrodynamic coefficients. Therefore, given the results presented in this thesis, the slenderbody approach could be quite useful to compute the slow-drift motions of a FOWT in long waves, which is the case, for instance, of analyzes considering extreme sea conditions. 
Moreover, since the software is also able to analyze aerodynamic and first-order hydrodynamic loads on both floating and fixed structures, it is an useful tool for other relevant problems.

\subsection{Future work}

Several lines of future work could follow this thesis, both concerning a deeper investigation of the slender-body approximation and further developments of the numerical tool, and the ones listed below are those that were considered to be the most relevant:

- The complementarity between the slender-body and Newman's approximation is not so easy to be exploited in time domain once body motions are taken into account. Some alternatives that may be investigated are to consider only the force components that contribute the most to the imaginary part of the force predicted with the slender-body approximation; take body motions into account in only one of the approximations, while the body is kept fixed to compute the loads with the other; or, perhaps, simply shift from one approximation to the other for wave components with $\lambda / D$ above a certain threshold;

- One major disadvantage of the slender-body approximation is that it is not well suited to evaluate the mean drifts of the body. However, if end effects and first-order motions are completely neglected, the analytical solution of the potential flow round a vertical cylinder can be obtained using the same procedure employed for the bottom mounted vertical cylinder (KIM; YUE, 1989, 1990). As this problem is more relevant for short waves, for which both end effects and first-order body motions are indeed frequently negligible, this may be a good approach to compute the mean loads and, perhaps, even the part of the difference-frequency forces that are missing from the slender-body approximation. Modifications would be needed, of course, for inclined cylinders;

- Though this work dealt exclusively with the difference-frequency second-order forces, a possible application of the software would be to analyze the sum-frequency loads on fixed structures, which is a relevant issue for fixed offshore wind turbines;

- As briefly discussed in Section 3.7, there are evidences in the literature suggesting that the simple quadratic drag term from Morison's equation is not sufficient to properly model the viscous drag in floating structures, which is corroborated by the results presented in this thesis. Investigating alternative force models to this part of the loads is, then, an important topic;

- A better understanding of the flow around the floater is necessary to establish good practices for the selection of the added mass and drag coefficients to be adopted 
in numerical simulations, which could hopefully be obtained though additional experimental campaigns and numerical simulations with CFD software. Forced oscillation tests considering the action of more than one frequency of motion, or performed under the action of waves instead of calm waters, could be very useful, and a formal assessment of uncertainties would be very welcome;

- Besides establishing the limitations of the slender-body approximation in terms of the parameters that describe a cylinder, such as done in Chapter 5, it would be useful to perform a similar assessment regarding the interactions between different cylinders that compose a structure, for instance in terms of the distance between two columns that is needed to obtain good results with the approximation;

- A substantial amount of work can be done to improve METiS as an engineering tool. The most critical at the moment would be the implementation of a dynamic mooring model; elasticity of blades and tower; and control of blades and rotor. 



\section{References}

AGARWAL, P.; MANUEL, L. Incorporating irregular nonlinear waves in coupled simulation and reliability studies of offshore wind turbines. Applied Ocean Research, v. 33, n. 3, p. 215$227,2011$.

AMARAL, G.; MEllo, P.; CARMO, L.; AlBERTO, I.; MALTA, E.; SIMOS, A.; FRANZINI, G.; SUZUKI, H.; GONÇALVES, R. Seakeeping Tests of a FOWT in Wind and Waves: An Analysis of Dynamic Coupling Effects and Their Impact on the Predictions of Pitch Motion Response. Journal of Marine Science and Engineering, v. 9, n. 2, p. 179, 2021.

AMARAL, G. A. Analytical assessment of the mooring system stiffness. 2020. MA thesis Universidade de São Paulo.

ANSYS. AQWA Reference Manual - Release 17.0. Canonsburg, PA, USA, 2017.

ARANHA, J. A. P.; FERNANDES, A. C. On the second-order slow drift force spectrum. Applied Ocean Research, v. 17, n. 5, p. 311-313, 1995.

BACHYNSKI, E. Design and dynamic analysis of tension leg platform wind turbines. 2014. PhD thesis - Norwegian University of Science and Technology.

BUREAU VERITAS. Hydrostar for Experts - User Manual. Neuilly-Sur-Seine, France, 2016 .

BURMESTER, S.; VAZ, G.; GUEYDON, S.; MOCTAR, O. el. Investigation of a semisubmersible floating wind turbine in surge decay using CFD. Ship Technology Research, v. 67, n. 1, p. 2-14, 2020.

BUTTERFIELD, S.; MUSIAL, W.; JONKMAN, J.; SCLAVOUNOS, P. Engineering challenges for floating offshore wind turbines. National Renewable Energy Lab.(NREL) - Golden, CO (United States), 2005. Presented at the 2005 Copenhagen Offshore Wind Conference.

CARMO, L. Aplicação da equação de Morison na modelagem hidrodinâmica de turbinas eólicas flutuantes. 2016. Undergraduate monograph - University of São Paulo.

CARMO, L.; CAMARGO, E.; SIMOS, A. Further discussion on the white-noise approach for predicting slow-drifts in FAST. In: ASME. $9^{\text {th }}$ International Offshore Wind Technical Conference. 2018. v. 51975, v001t01a026. 
CARMO, L.; MEllo, P.; MALTA, E.; FRAnZINI, G.; SIMOS, A.; GONÇALVES, R.; SUZUKI, H. Analysis of a FOWT model in bichromatic waves: an investigation on the effect of combined wave-frequency and slow motions on the calibration of drag and inertial force coefficients. In: ASME. $39^{\text {th }}$ International Conference on Ocean, Offshore and Arctic Engineering. Virtual, Online, 2020.

CERMelli, C.; LEROUX, C.; DOMÍNGUEZ, S.; PEIFFER, A. Experimental Measurements of WindFloat 1 Prototype Responses and Comparison With Numerical Model. In: ASME. $37^{\text {th }}$ International Conference on Ocean, Offshore and Arctic Engineering. Madrid, Spain, 2018.

CERMELLI, C.; RODDIER, D. Experimental and numerical investigation of the stabilizing effects of a water-entrapment plate on a deepwater minimal floating platform. In: ASME. $24^{\text {th }}$ International Conference on Offshore Mechanics and Arctic Engineering. Halkidiki, Greece, 2005.

COUllinG, A.; GOUPEE, A.; ROBERTSON, A.; JONKMAN, J. Importance of secondorder difference-frequency wave-diffraction forces in the validation of a FAST semisubmersible floating wind turbine model. In: ASME. $32^{\text {nd }}$ International Conference on Ocean, Offshore and Arctic Engineering. 2013. v. 55423, v008t09a019.

CRAIK, A. The origins of water wave theory. Annu. Rev. Fluid Mech., v. 36, p. 1-28, 2004.

CUMMINS, W. E. The impulse response function and ship motions. Technical Report 1661, David Taylor Model Basin, 1962.

DEAN, R.; DALRYMPLE, R. Water Wave Mechanics for Engineers and Scientists. World Scientific Publishing Company, 1991.

EMMERHOFF, O.; SCLAVOUNOS, P. The simulation of slow-drift motions of offshore structures. Applied Ocean Research, v. 18, n. 2-3, p. 55-64, 1996.

EQUINOR. How Hywind works. 2017. https : / / www . equinor . com/en/what - we do / hywind - where - the - wind - takes - us / hywind - up - close - and - personal . html. Accessed: 15/05/2019.

FALTINSEN, O. M. Sea loads on ships and offshore structures. Cambridge University Press, 1993.

FRANÇA, L. N. F.; MATSUMURA, A. Z. Mecânica geral. Editora Blucher, 2011.

GONÇALVES, R.; FRANZINI, G.; SIMOS, A.; GAY NETO, A.; MELlO, P.; CARMO, B.; NISHIMOTO, K.; MALTA, E.; VIEIRA, D.; CARMO, L.; AMARAL, G.; OLIVEIRA, M.; WATA, R.; HIRABAYASHI, S.; SUZUKI, H. A Brazil-Japan collaboration on a conceptual design of a floating offshore wind turbine for the São Paulo Coast. In: $27^{\text {th }}$ Int. Congress on Waterborn Transportation, Shipbuilding and Offshore Constructions. 2018. 
GUEYDON, S.; DUARTE, T.; JONKMAN, J. Comparison of second-order loads on a semisubmersible floating wind turbine. In: ASME. $33^{\text {th }}$ International Conference on Ocean, Offshore and Arctic Engineering. 2014. v. 45530, v09at09a024.

GWEC. Global Offshore Wind Report 2020. 2020.

GWEC. Global Wind Report 2019. 2020.

GWEC. Global Wind Report 2020. 2021.

HARVEY, A. L. World's first floating wind farm powers up off Scottish coast. Power, v. 161 , n. 12 , p. 12 , Dec. 2017.

HAVELOCK, T. The drifting force on a ship among waves. The London, Edinburgh, and Dublin Philosophical Magazine and Journal of Science, v. 33, n. 221, p. 467-475, 1942.

HAVELOCK, T. H. The pressure of water waves upon a fixed obstacle. Proceedings of the Royal Society of London, v. 175, n. 963, p. 409-421, 1940.

HEGSETH, J. M.; BACHYNSKI, E. E. A semi-analytical frequency domain model for efficient design evaluation of spar floating wind turbines. Marine Structures, v. 64, p. 186$210,2019$.

HESS, J. L.; SMITH, A. M. O. Calculation of potential flow about arbitrary bodies. Progress in Aerospace Sciences, 1967.

HITACHI. The wind from the future: Fukushima floating offshore wind farm demonstration project. Hitachi Review, v. 63, n. 3, p. 12-17, Mar. 2014.

HOOFT, J. P. Hydrodynamic aspects of semi-submersible platforms. 1972. PhD thesis Delft University of Technology.

ISAACSON, M. S. Nonlinear inertia forces on bodies. Journal of the Waterway, Port, Coastal and Ocean Division, v. 105, n. 3, 1979.

JAMES, R.; ROS, M. Floating offshore wind: market and technology review. The Carbon Trust - London, UK, 2015.

JOHN, F. On the motion of floating bodies II. Simple harmonic motions. Communications on pure and applied mathematics, Wiley Online Library, v. 3, n. 1, p. 45-101, 1950.

JONKMAN, J. Dynamics modeling and loads analysis of an offshore floating wind turbine. 2007. PhD thesis - University of Colorado Boulder.

JONKMAN, J.; BUHL, M. FAST User's Guide. Golden, CO, USA, 2005.

JONKMAN, J.; BUTTERFIELD, S.; MUSIAL, W.; SCOTT, G. Definition of a 5-MW reference wind turbine for offshore system development. 2009.

JONKMAN, J.; MUSIAL, W. Offshore Code Comparison Collaboration (OC3) for IEA Wind Task 23 offshore wind technology and deployment. Golden, CO, USA, 2010. 
JURADO, A.; BREDMOSE, H. Accelerated hydrodynamic analysis for spar buoys with second-order wave excitation. In: ASME. $39^{\text {th }}$ International Conference on Ocean, Offshore and Arctic Engineering. Virtual, Online, 2020.

KEUlEGAN, G.; CARPENTER, L. Forces on cylinders and plates in an oscillating fluid. Journal of Research of the National Bureau of Standards, v. 60, 1958.

KIM, M. H. Difference-frequency wave loads on a large body in multi-directional waves. Applied Ocean Research, v. 14, n. 6, p. 353-370, 1992.

KIM, M. H.; CHEN, W. Slender-body approximation for slowly-varying wave loads in multi-directional waves. Applied Ocean Research, v. 16, n. 3, p. 141-163, 1994.

KIM, M. H.; YUE, D. The complete second-order diffraction solution for an axisymmetric body - Part 1. Monochromatic incident waves. Journal of Fluid Mechanics, v. 200, p. 235264, 1989.

KIM, M. H.; YUE, D. The complete second-order diffraction solution for an axisymmetric body - Part 2. Bichromatic incident waves and body motions. Journal of Fluid Mechanics, v. 211, p. 557-593, 1990.

KORSMEYER, F. T.; BINGHAM, H. B.; NEWMAN, J. N. TIMIT - A panel-method program for transient wave-body interactions - Version 4.0. Cambridge, MA, USA, 1999.

KVITTEM, M. I.; MOAN, T. Frequency versus time domain fatigue analysis of a semisubmersible wind turbine tower. Journal of Offshore Mechanics and Arctic Engineering, v. 137, n. 1, 2015.

LEE, CH. WAMIT Theory Manual. Cambridge, MA, USA, 1995.

LI, H.; BACHYNSKI-POLIĆ, E. E. Analysis of difference-frequency wave loads and quadratic transfer functions on a restrained semi-submersible floating wind turbine. Ocean Engineering, v. 232, p. 109165, 2021.

LI, H.; BACHYNSKI-POLIĆ, E. E. Experimental and numerically obtained low-frequency radiation characteristics of the OC5-DeepCwind semisubmersible. Ocean Engineering, v. 232, p. 109130, 2021.

LI, H.; BACHYNSKI-POLIĆ, E. E. Validation and application of nonlinear hydrodynamics from CFD in an engineering model of a semi-submersible floating wind turbine. Marine Structures, v. 79, p. 103054, 2021.

LIGHTHILL, J. Fundamentals concerning wave loading on offshore structures. Journal of Fluid Mechanics, v. 173, p. 667-681, 1986.

LIGHTHILL, J. Waves and hydrodynamic loading. London, U.K., 1992.

LOPEZ-PAVON, C.; WATAI, R. A.; RUGGERI, F.; SIMOS, A.; SOUTO-IGLESIAS, A. Influence of wave induced second-order forces in semisubmersible FOWT mooring design. Journal of Offshore Mechanics and Arctic Engineering, v. 137, n. 3, 2015. 
MA, Q. W.; PATEL, M. H. On the non-linear forces acting on a floating spar platform in ocean waves. Applied Ocean Research, v. 23, n. 1, p. 29-40, 2001.

MACCAMY, R. C.; FUCHS, R. A. Wave forces on piles: A diffraction theory. Technical Memorandum No. 69, Beach Erosion Board, 1954.

MADSEN, O. Hydrodynamic force on circular cylinders. Applied Ocean Research, v. 8, n. 3, p. 151-155, 1986.

MANNERS, W. Hydrodynamic force on a moving circular cylinder submerged in a general fluid flow. Proceedings of the Royal Society of London. Series A: Mathematical and Physical Sciences, v. 438, n. 1903, p. 331-339, 1992.

MANNERS, W.; RAINEY, R. Hydrodynamic forces on fixed submerged cylinders. Proceedings of the Royal Society of London. Series A: Mathematical and Physical Sciences, v. 436, n. 1896, p. 13-32, 1992.

MANWELL, J.; MCGOWAN, J.; ROGERS, A. Wind energy explained: theory, design and application. John Wiley \& Sons, 2010.

MARUO, H. The excess resistance of a ship in rough seas. International Shipbuilding Progress, v. 4, n. 35, p. 337-345, 1957.

MATOS, V. L. F; SIMOS, A. N.; SPHAIER, S. H. Second-order resonant heave, roll and pitch motions of a deep-draft semi-submersible: Theoretical and experimental results. Ocean Engineering, v. 38, n. 17-18, p. 2227-2243, 2011.

MELLO, P.; CARNEIRO, M.; TANNURI, E.; KASSAB JR, F.; MARQUES, R.; ADAMOWSKI, J.; NISHIMOTO, K. A control and automation system for wave basins. Mechatronics, v. 23, n. 1, p. 94-107, 2013.

MELlO, P.; MALTA, E.; SILVA, R. da; CANDIDO, M.; CARMO, L.; ALBERTO, I.; FRANZINI, G.; SIMOS, A.; SUZUKI, H.; GONÇALVES, R. Influence of heave plates on the dynamics of a floating offshore wind turbine in waves. Journal of Marine Science and Technology, v. 26, p. 190-200, 2021.

MERRIFIELD, R. Offshore wind farms to test business in deep water. Horizon: the EU Research and Innovation Magazine, Oct. 2018. https://horizon-magazine.eu/ article/offshore-wind-farms-test-business-deep-water .html.

MOLIN, B. Second-order diffraction loads upon three-dimensional bodies. Applied Ocean Research, v. 1, n. 4, p. 197-202, 1979.

MOLIN, B. Second-order hydrodynamics applied to moored structures. In: $19^{\text {th }}$ WEGEMT School. 1993.

MOLIN, B.; CHEN, X. B. Approximations of the low-frequency second-order wave loads: Newman versus Rainey. Proc. 17th IWWWFB, Cambridge (UK), 2002. 
MORISON, J. R.; O'BRIEN, M. P.; JOHNSON, J. W.; SCHAAF, S. A. The force exerted by surface waves on piles. Journal of Petroleum Technology, v. 2, n. 05, p. 149-154, 1950. NEWMAN, J. N. Marine Hydrodynamics. MIT press, 1977.

NEWMAN, J. N. Second-order, slowly-varying forces on vessels in irregular waves. In: MECHANICAL ENGINEERING PUBLICATIONS LTD. PROC. Int. Symp. Dynamics of Marine Vehicles and Structures in Waves. London, UK, 1974.

NEWMAN, J. N. The theory of ship motions. In: ADVANCES in applied mechanics. 1979. v. 18. P. 221-283.

NING, S. A. A simple solution method for the blade element momentum equations with guaranteed convergence. Wind Energy, v. 17, n. 9, p. 1327-1345, 2014.

NREL. 2014-2015 Offshore Wind Technologies Market Report. 2015.

OGILVIE, T. F. Recent progress toward the understanding and prediction of ship motions. In: ONR. $5^{\text {th }}$ Symposium on Naval Hydrodynamics. Bergen, Norway, 1964.

ORCINA. OrcaFlex documentation (version 11.1c). 2021. Available from: <www.orcina. com/webhelp/OrcaFlex/Default.htm>.

PEGORARO, B. Modelagem aerodinâmica de turbinas eólicas flutuantes. 2018. MA thesis - University of São Paulo.

PESCE, C. Cálculo da Força de Deriva Sobre Corpos Cilíndricos Flutuantes Sujeitos à Ação de Ondas Aleatórias. 1984. MA thesis - University of São Paulo.

PESCE, C. P.; AMARAL, G. A.; FRANZINI, G. R. Mooring system stiffness: A general analytical formulation with an application to floating offshore wind turbines. In: AMERICAN SOCIETY OF MECHANICAL ENGINEERS. $9^{\text {th }}$ International Offshore Wind Technical Conference. 2018. v. 51975, v001t01a021.

PESCE, C.P.; ARANHA, J.A.P. O Espectro de Potência das Forças de Deriva sobre Corpos Cilíndricos Flutuantes em Ondas Aleatórias. Revista Brasileira de Engenharia, Caderno de Engenharia Naval, v. 2, p. 43-79, 1985.

PINKSTER, J. A. Low frequency second order wave exciting forces on floating structures. 1980. PhD thesis - Delft University of Technology.

PINKSTER, J. A. Mean and low frequency wave drifting forces on floating structures. Ocean Engineering, v. 6, p. 593-615, 1979.

PRESS, W. H.; TEUKOLSKY, S. A.; VETTERLING, W. T.; FLANNERY, B. P. Numerical recipes in C. 2. ed.: Cambridge University Press, 1992.

RAINEY, R. A new equation for calculating wave loads on offshore structures. Journal of Fluid Mechanics, v. 204, p. 295-324, 1989. 
RAINEY, R. Slender-body expressions for the wave load on offshore structures. Proceedings of the Royal Society of London. Series A: Mathematical and Physical Sciences, v. 450, p. 391-416, 1995.

REMERY, G.; HERMANS, A. The slow drift oscillations of a moored object in random seas. Society of Petroleum Engineers Journal, v. 12, n. 03, p. 191-198, 1972.

REN21. Renewables 2021: Global status report. 2021.

REZENDE, F. C.; CHEN, X. B.; FERREIRA, M. D. Second order roll motions for FPSO's operating in severe environmental conditions. In: ONEPETRO. OFFSHORE Technology Conference. 2007.

ROBERTSON, A.; JONKMAN, J.; MASCIOLA, M.; SONG, H.; GOUPEE, A.; COULlinG, A.; LUAN, C. Definition of the semisubmersible floating system for phase II of OC4. 2014. ROBERTSON, A.; JONKMAN, J.; VORPAHL, F.; POPKO, W.; QVIST, J.; FRØYD, L.; CHEN, X.; AZONA, J.; UZUNOGLU, E.; SOARES, C. G.; LUAN, C.; YUTONG, H.; PENGCHENG, F.; YDE, A.; LARSEN, T.; NICHOLS, J.; BUILS, R.; LEI, L.; NYGAARD, T.; MANOLAS, D.; HEEGE, A.; VATNE, S.; ORMBERG, H.; DUARTE, T.; GODREAU, C.; HANSEN, H.; NIELSEN, A.; RIBER, H.; LE CUNFF, C.; BEYER, F.; YAMAGUCHI, A.; JUNG, K.; SHIN, H.; SHI W.AND PARK, H.; ALVES, M; GUÉRINEL, M. Offshore Code Comparison Collaboration Continuation within IEA wind task 30: Phase II results regarding a floating semisubmersible wind system. In: ASME. $33^{\text {rd }}$ International Conference on Ocean, Offshore and Arctic Engineering. San Francisco, CA, USA, 2014.

ROBERTSON, A.; WENDT, F.; JONKMAN, J.; POPKO, W.; DAGHER, H.; GUEYDON, S.; QVIST, J.; VITTORI, F.; AZCONA, José; UZUNOGLU, E.; SOARES, C.; HARRIES, R.; YDE, A.; GALINOS, C.; HERMANS, K.; DE VAAL, J.; BOZONNET, P.; BOUY, L.; BAYATI, I.; BERGUA, R.; GALVAN, J.; MENDIKOA, I.; SANCHEZ, C.; SHIN, H.; OH, S.; MOLINS, C.; DEBRUYNE, Y. OC5 Project Phase II: Validation of Global Loads of the DeepCwind Floating Semisubmersible Wind Turbine. Energy Procedia, v. 137, p. 38-57, 2017. $14^{\text {th }}$ Deep Sea Offshore Wind R\&D Conference, EERA DeepWind.

RUGGERI, F. A higher order time domain panel method for linear and weakly non linear seakeeping problems. 2016. PhD thesis - University of São Paulo.

SANDERSON, C.; CURTIN, R. Armadillo: a template-based C ++ library for linear algebra. Journal of Open Source Software, v. 1, n. 2, p. 26, 2016.

SARPKAYA, T. Force on a circular cylinder in viscous oscillatory flow at low Keulegan-Carpenter numbers. Journal of Fluid Mechanics, Cambridge University Press, v. 165, p. $61-71,1986$.

SARPKAYA, T. On the force decompositions of Lighthill and Morison. Journal of Fluids and Structures, v. 15, n. 2, p. 227-233, 2001. 
SARPKAYA, T. Vortex shedding and resistance in harmonic flow about smooth and rough circular cylinders at high Reynolds numbers. 1976.

SARPKAYA, T. Wave forces on offshore structures. Cambridge university press, 2010.

SIMOS, A.; CARMO, L.; CAMARGO, E. On the use of the white-noise approximation for modelling the slow-drifts of a FOWT: An example using FAST. In: ASME. $37^{\text {th }}$ International Conference on Ocean, Offshore and Arctic Engineering. 2018.

SIMOS, A. N.; RUGGERI, F.; WATAI, R. A.; SOUTO-IGLESIAS, A.; LOPEZ-PAVON, C. Slow-drift of a floating wind turbine: An assessment of frequency-domain methods based on model tests. Renewable energy, v. 116, p. 133-154, 2018.

SKAARE, B. Development of the Hywind concept. In: ASME. $36^{\text {th }}$ International Conference on Ocean, Offshore and Arctic Engineering. Trondheim, Horway, 2017.

SOUZA, C. E. S.; BACHYNSKI, E. E. Changes in surge and pitch decay periods of floating wind turbines for varying wind speed. Ocean Engineering, v. 180, p. 223-237, 2019.

SOUZA, C. E. S.; HEGSETH, J. M.; BACHYNSKI, E. E. Frequency-dependent aerodynamic damping and inertia in linearized dynamic analysis of floating wind turbines. In: IOP PUBLISHING, 1. JOURNAL of Physics: Conference Series. 2020. v. 1452, p. 012040. STANSBERG, C. T.; GUDMESTAD, O. T.; HAVER, S. K. Kinematics under extreme waves. Journal of Offshore Mechanics and Arctic Engineering, v. 130, n. 2, 2008.

STOKES, G. On the theory of oscillatory waves. Transactions of the Cambridge Philosophical Society, v. 8, 1847.

SUYEHIRO, K. The drift of ships caused by rolling among waves. Trans. Inst. Naval Arch, v. 66, 1924.

TAYLOR, R. E.; RAINEY, R. C.; DAI, D. N. Non-Linear Hydrodynamic Analysis of TLP's in Extreme Waves: Slender Body and Diffraction Theories Compared. London, U.K., 1992.

TOM, N.; ROBERTSON, A.; JONKMAN, J.; WENDT, F.; BÖHM, M. Bichromatic Wave Selection for Validation of the Difference-Frequency Transfer Function for the OC6 Validation Campaign. In: ASME. $38^{\text {th }}$ International Conference on Ocean, Offshore and Arctic Engineering. 2019. v. 59353, v001t01a022.

VAN DER VEGT, J. J. Slinger gedrag van schepen. 1984.

VENUGOPAL, V.; VARYANI, K.; BARLTROP, N. Wave force coefficients for horizontally submerged rectangular cylinders. Ocean Engineering, v. 33, p. 1669-1704, 2006.

WAMIT, Inc. WAMIT User Manual - Versions 6.2, 6.2PC, 6.2S, 6.1S-PC. Chestnut Hill, MA, USA, 2004. 
WANG, E.; ZHANG, Q.; SHEN, B.; ZHANG, G.; LU, X.; WU, Q.; WANG, Y. Intel Math Kernel Library. In: HIGH-PERFORMANCE Computing on the Intel@ Xeon Phi ${ }^{\mathrm{TM}}$. Springer, 2014. P. 167-188.

WATAI, R. A time-domain boundary elements method for the seakeeping analysis of offshore systems. 2015. PhD thesis - University of São Paulo.

WEHAUSEN, J. The motion of floating bodies. Annual Review of Fluid Mechanics, v. 3, n. 1, p. 237-268, 1971.

WHEELER, J.D. Method for Calculating Forces Produced by Irregular Waves. Journal of Petroleum Technology, v. 22, n. 03, p. 359-367, Mar. 1970.

ZHU, X. Irregular frequency removal from the boundary integral equation for the wave-body problem. 1994. MA thesis - Massachusetts Institute of Technology. 



\section{APPENDIX A - Added mass matrix}

As discussed in Section 3.3.4, the hydrodynamic forces and moments due to the acceleration of a point on the cylinder axis are divided in two parts: one that is due to the centripetal acceleration, which depends only on the rotational velocity $\dot{\boldsymbol{\xi}}_{R}$ of the body, and another that is linearly proportional to the acceleration $\ddot{\boldsymbol{\xi}}$. The first part is kept with the other hydrodynamic forces on the right side of the equations of motion, while the second one is included as a matrix that is added to the inertia of the body. The objective of this appendix is to explain how this matrix is calculated, starting by the expression of the force that depends linearly on the acceleration of of the body:

$$
\mathbf{F}_{\ddot{x}_{l}}=-\frac{\pi D^{2}}{4} \rho C_{a} \int_{0}^{L_{0}}\left[\ddot{x}_{l}(z) \mathbf{e}_{1}+\ddot{y}_{l}(z) \mathbf{e}_{2}\right] \mathrm{d} z-\rho C_{a V} V_{R} \ddot{z}_{l} \mathbf{e}_{3}
$$

and the respective moment

$$
\begin{aligned}
\mathbf{M}_{\ddot{x}_{l}}= & -\frac{\pi D^{2}}{4} \rho C_{a} \int_{0}^{L_{0}}\left[\mathbf{x}_{B}(z)-\mathbf{x}_{G}\right] \wedge\left[\ddot{x}_{l}(z) \mathbf{e}_{1}+\ddot{y}_{l}(z) \mathbf{e}_{2}\right] \mathrm{d} z \\
& -\rho C_{a V} V_{R}\left[\mathbf{x}_{B}(0)-\mathbf{x}_{G}\right] \wedge \ddot{z}_{l} \mathbf{e}_{3}
\end{aligned}
$$

in which the same notation of Chapter 3 is adopted, with the origin of the local coordinate system of the cylinder, $O$, taken at its bottom. The linear component of the acceleration, $\ddot{\mathbf{x}}_{l}(z)$, is given by:

$$
\begin{aligned}
\ddot{\mathbf{x}}_{l}(z) & =\ddot{\boldsymbol{\xi}}_{T}+\ddot{\boldsymbol{\xi}}_{R} \wedge\left[\mathbf{x}_{B}(z)-\mathbf{x}_{G}\right] \\
& =\left[\ddot{\xi}_{1}+\ddot{\xi}_{5}\left[Z_{B}(z)-Z_{G}\right]-\ddot{\xi}_{6}\left[Y_{B}(z)-Y_{G}\right]\right] \mathbf{E}_{1} \\
& +\left[\ddot{\xi}_{2}+\ddot{\xi}_{6}\left[X_{B}(z)-X_{G}\right]-\ddot{\xi}_{4}\left[Z_{B}(z)-Z_{G}\right]\right] \mathbf{E}_{2} \\
& +\left[\ddot{\xi}_{3}+\ddot{\xi}_{4}\left[Y_{B}(z)-Y_{G}\right]-\ddot{\xi}_{5}\left[X_{B}(z)-X_{G}\right]\right] \mathbf{E}_{3}
\end{aligned}
$$

however, as Equations A.1 and A.2 are written in terms of the components in the local reference frame, it is necessary to project Equation A.3 onto $\mathbf{e}_{1}, \mathbf{e}_{2}$, and $\mathbf{e}_{3}$ :

$$
\ddot{x}_{l}(z)=\ddot{\mathbf{x}}_{l}(z) \cdot \mathbf{e}_{1} \quad \ddot{y}_{l}(z)=\ddot{\mathbf{x}}_{l}(z) \cdot \mathbf{e}_{2} \quad \ddot{z}_{l}(z)=\ddot{\mathbf{x}}_{l}(z) \cdot \mathbf{e}_{3}
$$

For clarity, $\mathbf{F}_{\ddot{x}_{l}}$ and $\mathbf{M}_{\ddot{x}_{l}}$ are broken down in two components: one due to the forces along the length of the cylinder, $\mathbf{F}_{\ddot{x}_{l}, P}$ and $\mathbf{M}_{\ddot{x}_{l}, P}$, and one due to its extremities, $\mathbf{F}_{\ddot{x}_{l}, b}$, $\mathbf{M}_{\ddot{x}_{l}, b}, \mathbf{F}_{\ddot{x}_{l}, t}, \mathbf{M}_{\ddot{x}_{l}, t}$ (standing for bottom and top, respectively). In the end, the added mass matrix is the sum of matrices corresponding to each of these components:

$$
\mathbb{A}=\mathbb{A}_{P}+\mathbb{A}_{b}+\mathbb{A}_{t}
$$


which have very similar expressions, hence the procedure will be illustrated for $\mathbb{A}_{P}$ only.

The linear added mass of each cylinder section, considered to be constant along its length, is:

$$
\Lambda=\frac{\pi D^{2}}{4} \rho C_{a}
$$

The forces and moments can be obtained by replacing Equations A.3 and A.4 in Equations A.1 and A.2. The components of the forces and moments in the directions $X$, $Y$, and $Z$ are:

$$
\begin{aligned}
\mathbf{F}_{\ddot{x}_{l}, P} \cdot \mathbf{E}_{1} & =-\Lambda \int_{0}^{L}\left\{\left[\ddot{\xi}_{1}+\ddot{\xi}_{5}\left[Z_{B}(Z)-Z_{G}\right]-\ddot{\xi}_{6}\left[Y_{B}(z)-Y_{G}\right]\right]\left[\left(\mathbf{e}_{1} \cdot \mathbf{E}_{1}\right)^{2}+\left(\mathbf{e}_{2} \cdot \mathbf{E}_{1}\right)^{2}\right]\right. \\
& +\left[\ddot{\xi}_{2}+\ddot{\xi}_{6}\left[X_{B}(z)-X_{G}\right]-\ddot{\xi}_{4}\left[Z_{B}(z)-Z_{G}\right]\right]\left[\left(\mathbf{e}_{1} \cdot \mathbf{E}_{2}\right)\left(\mathbf{e}_{1} \cdot \mathbf{E}_{1}\right)+\left(\mathbf{e}_{2} \cdot \mathbf{E}_{2}\right)\left(\mathbf{e}_{2} \cdot \mathbf{E}_{1}\right)\right](\mathrm{A} .7) \\
& \left.+\left[\ddot{\xi}_{3}+\ddot{\xi}_{4}\left[Y_{B}(z)-Y_{G}\right]-\ddot{\xi}_{5}\left[X_{B}(z)-X_{G}\right]\right]\left[\left(\mathbf{e}_{1} \cdot \mathbf{E}_{3}\right)\left(\mathbf{e}_{1} \cdot \mathbf{E}_{1}\right)+\left(\mathbf{e}_{2} \cdot \mathbf{E}_{3}\right)\left(\mathbf{e}_{2} \cdot \mathbf{E}_{1}\right)\right]\right\} \mathrm{d} z \\
\mathbf{F}_{\ddot{x}_{l}, P} \cdot \mathbf{E}_{2} & =-\Lambda \int_{0}^{L}\left\{\left[\ddot{\xi}_{1}+\ddot{\xi}_{5}\left[Z_{B}(z)-Z_{G}\right]-\ddot{\xi}_{6}\left[Y_{B}(z)-Y_{G}\right]\right]\left[\left(\mathbf{e}_{1} \cdot \mathbf{E}_{1}\right)\left(\mathbf{e}_{1} \cdot \mathbf{E}_{2}\right)+\left(\mathbf{e}_{2} \cdot \mathbf{E}_{1}\right)\left(\mathbf{e}_{2} \cdot \mathbf{E}_{2}\right)\right]\right. \\
& +\left[\ddot{\xi}_{2}+\ddot{\xi}_{6}\left[X_{B}(z)-X_{G}\right]-\ddot{\xi}_{4}\left[Z_{B}(z)-Z_{G}\right]\right]\left[\left(\mathbf{e}_{1} \cdot \mathbf{E}_{2}\right)^{2}+\left(\mathbf{e}_{2} \cdot \mathbf{E}_{2}\right)^{2}\right] \\
& \left.+\left[\ddot{\xi}_{3}+\ddot{\xi}_{4}\left[Y_{B}(z)-Y_{G}\right]-\ddot{\xi}_{5}\left[X_{B}(z)-X_{G}\right]\right]\left[\left(\mathbf{e}_{1} \cdot \mathbf{E}_{3}\right)\left(\mathbf{e}_{1} \cdot \mathbf{E}_{2}\right)+\left(\mathbf{e}_{2} \cdot \mathbf{E}_{3}\right)\left(\mathbf{e}_{2} \cdot \mathbf{E}_{2}\right)\right]\right\} \mathrm{d} z \\
& \left.+\left[\ddot{\xi}_{3}+\ddot{\xi}_{4}\left[Y_{B}(z)-Y_{G}\right]-\ddot{\xi}_{5}\left[X_{B}(z)-X_{G}\right]\right]\left[\left(\mathbf{e}_{1} \cdot \mathbf{E}_{3}\right)^{2}+\left(\mathbf{e}_{2} \cdot \mathbf{E}_{3}\right)^{2}\right]\right\} \mathrm{d} z \\
& +\left[\ddot{\xi}_{2}+\ddot{\xi}_{6}\left[X_{B}(z)-X_{G}\right]-\ddot{\xi}_{4}\left[Z_{B}(z)-Z_{G}\right]\right]\left[\left(\mathbf{e}_{1} \cdot \mathbf{E}_{2}\right)\left(\mathbf{e}_{1} \cdot \mathbf{E}_{3}\right)+\left(\mathbf{e}_{2} \cdot \mathbf{E}_{2}\right)\left(\mathbf{e}_{2} \cdot \mathbf{E}_{3}\right)\right](\mathrm{A} .9) \\
& =-\Lambda \int_{0}^{L}\left\{\left[\ddot{\xi}_{1}+\ddot{\xi}_{5}\left[Z_{B}(z)-Z_{G}\right]-\ddot{\xi}_{6}\left[Y_{B}(z)-Y_{G}\right]\right]\left[\left(\mathbf{e}_{1} \cdot \mathbf{E}_{1}\right)\left(\mathbf{e}_{1} \cdot \mathbf{E}_{3}\right)+\left(\mathbf{e}_{2} \cdot \mathbf{E}_{1}\right)\left(\mathbf{e}_{2} \cdot \mathbf{E}_{3}\right)\right]\right. \\
& \\
& \\
& \\
&
\end{aligned}
$$

where $L$ is the wetted length of the cylinder. If the mean position of the body is considered, then $L=L_{0}$, while $L(t)=L_{0}+L^{(1)}(t)$ if the instantaneous first-order position of the body is considered. The former results in the matrix $\mathbb{A}$, while the latter yields the time varying added mass matrix $\mathbb{A}_{T}^{(1)}$, both introduced in Chapter 3.

For the moments $\mathbf{M}_{\ddot{x}_{l}}$, it is still necessary to evaluate the following cross product:

$$
\begin{aligned}
{\left[\mathbf{x}_{B}(z)-\mathbf{x}_{G}\right] \wedge \ddot{\mathbf{x}}_{P}(z) } & =\left[\left[\left(Y_{B}(z)-Y_{G}\right) \mathbf{E}_{3}-\left(Z_{B}(z)-Z_{G}\right) \mathbf{E}_{2}\right] \cdot \ddot{\mathbf{x}}_{P}(z)\right] \mathbf{E}_{1} \\
& +\left[\left[\left(Z_{B}(z)-Z_{G}\right) \mathbf{E}_{1}-\left(X_{B}(z)-X_{G}\right) \mathbf{E}_{3}\right] \cdot \ddot{\mathbf{x}}_{P}(z)\right] \mathbf{E}_{2} \\
& +\left[\left[\left(X_{B}(z)-X_{G}\right) \mathbf{E}_{2}-\left(Y_{B}(z)-Y_{G}\right) \mathbf{E}_{1}\right] \cdot \ddot{\mathbf{x}}_{P}(z)\right] \mathbf{E}_{3}
\end{aligned}
$$


with $\ddot{\mathbf{x}}_{P}(z)=\ddot{x}_{l}(z) \mathbf{e}_{1}+\ddot{y}_{l}(z) \mathbf{e}_{2}$. This results in the following expressions:

$$
\begin{aligned}
\mathbf{M}_{\ddot{x}_{l}, P} \cdot \mathbf{E}_{1} & =-\Lambda \int_{0}^{L}\left[Y_{B}(z)-Y_{G}\right]\left\{\left[\ddot{\xi}_{3}+\ddot{\xi}_{4}\left[Y_{B}(z)-Y_{G}\right]-\ddot{\xi}_{5}\left[X_{B}(z)-X_{G}\right]\right]\left[\left(\mathbf{e}_{1} \cdot \mathbf{E}_{3}\right)^{2}+\left(\mathbf{e}_{2} \cdot \mathbf{E}_{3}\right)^{2}\right]\right. \\
& +\left[\ddot{\xi}_{2}+\ddot{\xi}_{6}\left[X_{B}(z)-X_{G}\right]-\ddot{\xi}_{4}\left[Z_{B}(z)-Z_{G}\right]\right]\left[\left(\mathbf{e}_{1} \cdot \mathbf{E}_{2}\right)\left(\mathbf{e}_{1} \cdot \mathbf{E}_{3}\right)+\left(\mathbf{e}_{2} \cdot \mathbf{E}_{2}\right)\left(\mathbf{e}_{2} \cdot \mathbf{E}_{3}\right)\right] \\
& \left.+\left[\ddot{\xi}_{1}+\ddot{\xi}_{5}\left[Z_{B}(z)-Z_{G}\right]-\ddot{\xi}_{6}\left[Y_{B}(z)-Y_{G}\right]\right]\left[\left(\mathbf{e}_{1} \cdot \mathbf{E}_{1}\right)\left(\mathbf{e}_{1} \cdot \mathbf{E}_{3}\right)+\left(\mathbf{e}_{2} \cdot \mathbf{E}_{1}\right)\left(\mathbf{e}_{2} \cdot \mathbf{E}_{3}\right)\right]\right\} \\
& +\left[\ddot{\xi}_{1}+\ddot{\xi}_{5}\left[Z_{B}(z)-Z_{G}\right]-\ddot{\xi}_{6}\left[Y_{B}(z)-Y_{G}\right]\right]\left[\left(\mathbf{e}_{1} \cdot \mathbf{E}_{1}\right)\left(\mathbf{e}_{1} \cdot \mathbf{E}_{2}\right)+\left(\mathbf{e}_{2} \cdot \mathbf{E}_{1}\right)\left(\mathbf{e}_{2} \cdot \mathbf{E}_{2}\right)\right] \\
& +\left[\ddot{\xi}_{3}+\ddot{\xi}_{4}\left[Y_{B}(z)-Y_{G}\right]-\ddot{\xi}_{5}\left[X_{B}(z)-X_{G}\right]-\ddot{\xi}_{4}\left[Z_{B}(z)-Z_{G}\right]\right]\left[\left(\mathbf{e}_{1} \cdot \mathbf{E}_{2}\right)^{2}+\left(\mathbf{e}_{2} \cdot \mathbf{E}_{2}\right)^{2}\right] \\
&
\end{aligned}
$$

$$
\begin{aligned}
\mathbf{M}_{\ddot{x}_{l}, P} \cdot \mathbf{E}_{2}= & -\Lambda \int_{0}^{L}\left[Z_{B}(z)-Z_{G}\right]\left\{\left[\ddot{\xi}_{1}+\ddot{\xi}_{5}\left[Z_{B}(z)-Z_{G}\right]-\ddot{\xi}_{6}\left[Y_{B}(z)-Y_{G}\right]\right]\left[\left(\mathbf{e}_{1} \cdot \mathbf{E}_{1}\right)^{2}+\left(\mathbf{e}_{2} \cdot \mathbf{E}_{1}\right)^{2}\right]\right. \\
& +\left[\ddot{\xi}_{2}+\ddot{\xi}_{6}\left[X_{B}(z)-X_{G}\right]-\ddot{\xi}_{4}\left[Z_{B}(z)-Z_{G}\right]\right]\left[\left(\mathbf{e}_{1} \cdot \mathbf{E}_{2}\right)\left(\mathbf{e}_{1} \cdot \mathbf{E}_{1}\right)+\left(\mathbf{e}_{2} \cdot \mathbf{E}_{2}\right)\left(\mathbf{e}_{2} \cdot \mathbf{E}_{1}\right)\right] \\
& \left.+\left[\ddot{\xi}_{3}+\ddot{\xi}_{4}\left[Y_{B}(z)-Y_{G}\right]-\ddot{\xi}_{5}\left[X_{B}(z)-X_{G}\right]\right]\left[\left(\mathbf{e}_{1} \cdot \mathbf{E}_{3}\right)\left(\mathbf{e}_{1} \cdot \mathbf{E}_{1}\right)+\left(\mathbf{e}_{2} \cdot \mathbf{E}_{3}\right)\left(\mathbf{e}_{2} \cdot \mathbf{E}_{1}\right)\right]\right\} \\
& \quad-\left[X_{B}(z)-X_{G}\right]\left\{\left[\ddot{\xi}_{3}+\ddot{\xi}_{4}\left[Y_{B}(z)-Y_{G}\right]-\ddot{\xi}_{5}\left[X_{B}(z)-X_{G}\right]\right]\left[\left(\mathbf{e}_{1} \cdot \mathbf{E}_{3}\right)^{2}+\left(\mathbf{e}_{2} \cdot \mathbf{E}_{3}\right)^{2}\right]\right. \\
& +\left[\ddot{\xi}_{2}+\ddot{\xi}_{6}\left[X_{B}(z)-X_{G}\right]-\ddot{\xi}_{5}\left[Z_{B}(z)-Z_{G}\right]\right]\left[\left(\mathbf{e}_{1} \cdot \mathbf{E}_{2}\right)\left(\mathbf{e}_{1} \cdot \mathbf{E}_{3}\right)+\left(\mathbf{e}_{2} \cdot \mathbf{E}_{2}\right)\left(\mathbf{e}_{2} \cdot \mathbf{E}_{3}\right)\right] \\
&
\end{aligned}
$$

$$
\begin{aligned}
\mathbf{M}_{\ddot{x}_{l}, P} \cdot \mathbf{E}_{3} & =-\Lambda \int_{0}^{L}\left[X_{B}(z)-X_{G}\right]\left\{\left[\ddot{\xi}_{2}+\ddot{\xi}_{6}\left[X_{B}(z)-X_{G}\right]-\ddot{\xi}_{4}\left[Z_{B}(z)-Z_{G}\right]\right]\left[\left(\mathbf{e}_{1} \cdot \mathbf{E}_{2}\right)^{2}+\left(\mathbf{e}_{2} \cdot \mathbf{E}_{2}\right)^{2}\right]\right. \\
+ & +\left[\ddot{\xi}_{1}+\ddot{\xi}_{5}\left[Z_{B}(z)-Z_{G}\right]-\ddot{\xi}_{6}\left[Y_{B}(z)-Y_{G}\right]\right]\left[\left(\mathbf{e}_{1} \cdot \mathbf{E}_{1}\right)\left(\mathbf{e}_{1} \cdot \mathbf{E}_{2}\right)+\left(\mathbf{e}_{2} \cdot \mathbf{E}_{1}\right)\left(\mathbf{e}_{2} \cdot \mathbf{E}_{2}\right)\right] \\
+ & {\left.\left[\ddot{\xi}_{3}+\ddot{\xi}_{4}\left[Y_{B}(z)-Y_{G}\right]-\ddot{\xi}_{5}\left[X_{B}(z)-X_{G}\right]\right]\left[\left(\mathbf{e}_{1} \cdot \mathbf{E}_{3}\right)\left(\mathbf{e}_{1} \cdot \mathbf{E}_{2}\right)+\left(\mathbf{e}_{2} \cdot \mathbf{E}_{3}\right)\left(\mathbf{e}_{2} \cdot \mathbf{E}_{2}\right)\right]\right\} } \\
& -\left[Y_{B}(z)-Y_{G}\right]\left\{\left[\ddot{\xi}_{1}+\ddot{\xi}_{5}\left[Z_{B}(z)-Z_{G}\right]-\ddot{\xi}_{6}\left[Y_{B}(z)-Y_{G}\right]\right]\left[\left(\mathbf{e}_{1} \cdot \mathbf{E}_{1}\right)^{2}+\left(\mathbf{e}_{2} \cdot \mathbf{E}_{1}\right)^{2}\right]\right.
\end{aligned}
$$




$$
\begin{aligned}
& +\left[\ddot{\xi}_{2}+\ddot{\xi}_{6}\left[X_{B}(z)-X_{G}\right]-\ddot{\xi}_{4}\left[Z_{B}(z)-Z_{G}\right]\right]\left[\left(\mathbf{e}_{1} \cdot \mathbf{E}_{2}\right)\left(\mathbf{e}_{1} \cdot \mathbf{E}_{1}\right)+\left(\mathbf{e}_{2} \cdot \mathbf{E}_{2}\right)\left(\mathbf{e}_{2} \cdot \mathbf{E}_{1}\right)\right] \\
& \left.+\left[\ddot{\xi}_{3}+\ddot{\xi}_{4}\left[Y_{B}(z)-Y_{G}\right]-\ddot{\xi}_{5}\left[X_{B}(z)-X_{G}\right]\right]\left[\left(\mathbf{e}_{1} \cdot \mathbf{E}_{3}\right)\left(\mathbf{e}_{1} \cdot \mathbf{E}_{1}\right)+\left(\mathbf{e}_{2} \cdot \mathbf{E}_{3}\right)\left(\mathbf{e}_{2} \cdot \mathbf{E}_{1}\right)\right]\right\} \mathrm{d} z
\end{aligned}
$$

It is possible to write the forces and moments above as $\mathcal{F}_{\ddot{x}_{l}, P}=-\mathbb{A}_{P} \ddot{\boldsymbol{\xi}}$, with the different terms of $\mathbb{A}_{P}=A_{p q}^{[P]}$ given by:

$$
\begin{aligned}
& A_{p q}^{[P]}=\Lambda L\left[\left(\mathbf{e}_{1} \cdot \mathbf{E}_{p}\right)\left(\mathbf{e}_{1} \cdot \mathbf{E}_{q}\right)+\left(\mathbf{e}_{2} \cdot \mathbf{E}_{p}\right)\left(\mathbf{e}_{2} \cdot \mathbf{E}_{q}\right)\right] \mathrm{d} s \quad \text { for } p, q=1,2,3 \\
& A_{44}^{[P]}=\Lambda \int_{0}^{L}\left\{\left[Y_{B}(z)-Y_{G}\right]^{2}\left[\left(\mathbf{e}_{1} \cdot \mathbf{E}_{3}\right)^{2}+\left(\mathbf{e}_{2} \cdot \mathbf{E}_{3}\right)^{2}\right]\right. \\
& +\left[Z_{B}(z)-Z_{G}\right]^{2}\left[\left(\mathbf{e}_{1} \cdot \mathbf{E}_{2}\right)^{2}+\left(\mathbf{e}_{2} \cdot \mathbf{E}_{2}\right)^{2}\right] \\
& \left.-2\left[Y_{B}(z)-Y_{G}\right]\left[Z_{B}(z)-Z_{G}\right]\left[\left(\mathbf{e}_{1} \cdot \mathbf{E}_{2}\right)\left(\mathbf{e}_{1} \cdot \mathbf{E}_{3}\right)+\left(\mathbf{e}_{2} \cdot \mathbf{E}_{2}\right)\left(\mathbf{e}_{2} \cdot \mathbf{E}_{3}\right)\right]\right\} \mathrm{d} z \\
& A_{55}^{[P]}=\Lambda \int_{0}^{L}\left\{\left[X_{B}(z)-X_{G}\right]^{2}\left[\left(\mathbf{e}_{1} \cdot \mathbf{E}_{3}\right)^{2}+\left(\mathbf{e}_{2} \cdot \mathbf{E}_{3}\right)^{2}\right]\right. \\
& +\left[Z_{B}(z)-Z_{G}\right]^{2}\left[\left(\mathbf{e}_{1} \cdot \mathbf{E}_{1}\right)^{2}+\left(\mathbf{e}_{2} \cdot \mathbf{E}_{1}\right)^{2}\right] \\
& \left.-2\left[X_{B}(z)-X_{G}\right]\left[Z_{B}(z)-Z_{G}\right]\left[\left(\mathbf{e}_{1} \cdot \mathbf{E}_{1}\right)\left(\mathbf{e}_{1} \cdot \mathbf{E}_{3}\right)+\left(\mathbf{e}_{2} \cdot \mathbf{E}_{1}\right)\left(\mathbf{e}_{2} \cdot \mathbf{E}_{3}\right)\right]\right\} \mathrm{d} z \\
& A_{66}^{[P]}=\Lambda \int_{0}^{L}\left\{\left[X_{B}(z)-X_{G}\right]^{2}\left[\left(\mathbf{e}_{1} \cdot \mathbf{E}_{2}\right)^{2}+\left(\mathbf{e}_{2} \cdot \mathbf{E}_{2}\right)^{2}\right]\right. \\
& +\left[Y_{B}(z)-Y_{G}\right]^{2}\left[\left(\mathbf{e}_{1} \cdot \mathbf{E}_{1}\right)^{2}+\left(\mathbf{e}_{2} \cdot \mathbf{E}_{1}\right)^{2}\right] \\
& \left.-2\left[X_{B}(z)-X_{G}\right]\left[Y_{B}(z)-Y_{G}\right]\left[\left(\mathbf{e}_{1} \cdot \mathbf{E}_{1}\right)\left(\mathbf{e}_{1} \cdot \mathbf{E}_{2}\right)+\left(\mathbf{e}_{2} \cdot \mathbf{E}_{1}\right)\left(\mathbf{e}_{2} \cdot \mathbf{E}_{2}\right)\right]\right\} \mathrm{d} z \\
& A_{14}^{[P]}=A_{41}^{[P]}=\Lambda \int_{0}^{L}\left\{\left[Y_{B}(z)-Y_{G}\right]\left[\left(\mathbf{e}_{1} \cdot \mathbf{E}_{1}\right)\left(\mathbf{e}_{1} \cdot \mathbf{E}_{3}\right)+\left(\mathbf{e}_{2} \cdot \mathbf{E}_{1}\right)\left(\mathbf{e}_{2} \cdot \mathbf{E}_{3}\right)\right]\right. \\
& \left.-\left[Z_{B}(z)-Z_{G}\right]\left[\left(\mathbf{e}_{1} \cdot \mathbf{E}_{1}\right)\left(\mathbf{e}_{1} \cdot \mathbf{E}_{2}\right)+\left(\mathbf{e}_{2} \cdot \mathbf{E}_{1}\right)\left(\mathbf{e}_{2} \cdot \mathbf{E}_{2}\right)\right]\right\} \mathrm{d} z \\
& A_{15}^{[P]}=A_{51}^{[P]}=\Lambda \int_{0}^{L}\left\{\left[Z_{B}(z)-Z_{G}\right]\left[\left(\mathbf{e}_{1} \cdot \mathbf{E}_{1}\right)^{2}+\left(\mathbf{e}_{2} \cdot \mathbf{E}_{1}\right)^{2}\right]\right. \\
& \left.-\left[X_{B}(z)-X_{G}\right]\left[\left(\mathbf{e}_{1} \cdot \mathbf{E}_{1}\right)\left(\mathbf{e}_{1} \cdot \mathbf{E}_{3}\right)+\left(\mathbf{e}_{2} \cdot \mathbf{E}_{1}\right)\left(\mathbf{e}_{2} \cdot \mathbf{E}_{3}\right)\right]\right\} \mathrm{d} z \\
& A_{16}^{[P]}=A_{61}^{[P]}=\Lambda \int_{0}^{L}\left\{\left[X_{B}(z)-X_{G}\right]\left[\left(\mathbf{e}_{1} \cdot \mathbf{E}_{1}\right)\left(\mathbf{e}_{1} \cdot \mathbf{E}_{2}\right)+\left(\mathbf{e}_{2} \cdot \mathbf{E}_{1}\right)\left(\mathbf{e}_{2} \cdot \mathbf{E}_{2}\right)\right]\right. \\
& \left.-\left[Y_{B}(z)-Y_{G}\right]\left[\left(\mathbf{e}_{1} \cdot \mathbf{E}_{1}\right)^{2}+\left(\mathbf{e}_{2} \cdot \mathbf{E}_{1}\right)^{2}\right]\right\} \mathrm{d} z
\end{aligned}
$$




$$
\begin{gathered}
A_{24}^{[P]}=A_{42}^{[P]}=\Lambda \int_{0}^{L}\left\{\left[Y_{B}(z)-Y_{G}\right]\left[\left(\mathbf{e}_{1} \cdot \mathbf{E}_{2}\right)\left(\mathbf{e}_{1} \cdot \mathbf{E}_{3}\right)+\left(\mathbf{e}_{2} \cdot \mathbf{E}_{2}\right)\left(\mathbf{e}_{2} \cdot \mathbf{E}_{3}\right)\right]\right. \\
\left.-\left[Z_{B}(z)-Z_{G}\right]\left[\left(\mathbf{e}_{1} \cdot \mathbf{E}_{2}\right)^{2}+\left(\mathbf{e}_{2} \cdot \mathbf{E}_{2}\right)^{2}\right]\right\} \mathrm{d} z
\end{gathered}
$$

$$
\begin{aligned}
A_{25}^{[P]}=A_{52}^{[P]}=\Lambda \int_{0}^{L}\{ & {\left[Z_{B}(z)-Z_{G}\right]\left[\left(\mathbf{e}_{1} \cdot \mathbf{E}_{1}\right)\left(\mathbf{e}_{1} \cdot \mathbf{E}_{2}\right)+\left(\mathbf{e}_{2} \cdot \mathbf{E}_{1}\right)\left(\mathbf{e}_{2} \cdot \mathbf{E}_{2}\right)\right] } \\
& \left.\quad-\left[X_{B}(z)-X_{G}\right]\left[\left(\mathbf{e}_{1} \cdot \mathbf{E}_{2}\right)\left(\mathbf{e}_{1} \cdot \mathbf{E}_{3}\right)+\left(\mathbf{e}_{2} \cdot \mathbf{E}_{2}\right)\left(\mathbf{e}_{2} \cdot \mathbf{E}_{3}\right)\right]\right\} \mathrm{d} z
\end{aligned}
$$$$
A_{26}^{[P]}=A_{62}^{[P]}=\Lambda \int_{0}^{L}\left\{\left[X_{B}(z)-X_{G}\right]\left[\left(\mathbf{e}_{1} \cdot \mathbf{E}_{2}\right)^{2}+\left(\mathbf{e}_{2} \cdot \mathbf{E}_{2}\right)^{2}\right]\right.
$$$$
\left.-\left[Y_{B}(z)-Y_{G}\right]\left[\left(\mathbf{e}_{1} \cdot \mathbf{E}_{1}\right)\left(\mathbf{e}_{1} \cdot \mathbf{E}_{2}\right)+\left(\mathbf{e}_{2} \cdot \mathbf{E}_{1}\right)\left(\mathbf{e}_{2} \cdot \mathbf{E}_{2}\right)\right]\right\} \mathrm{d} z
$$

$$
\begin{aligned}
A_{34}^{[P]}=A_{43}^{[P]}=\Lambda \int_{0}^{L}\{ & {\left[Y_{B}(z)-Y_{G}\right]\left[\left(\mathbf{e}_{1} \cdot \mathbf{E}_{3}\right)^{2}+\left(\mathbf{e}_{2} \cdot \mathbf{E}_{3}\right)^{2}\right] } \\
& \left.\quad-\left[Z_{B}(z)-Z_{G}\right]\left[\left(\mathbf{e}_{1} \cdot \mathbf{E}_{2}\right)\left(\mathbf{e}_{1} \cdot \mathbf{E}_{3}\right)+\left(\mathbf{e}_{2} \cdot \mathbf{E}_{2}\right)\left(\mathbf{e}_{2} \cdot \mathbf{E}_{3}\right)\right]\right\} \mathrm{d} z
\end{aligned}
$$$$
A_{35}^{[P]}=A_{53}^{[P]}=\Lambda \int_{0}^{L}\left\{\left[Z_{B}(z)-Z_{G}\right]\left[\left(\mathbf{e}_{1} \cdot \mathbf{E}_{1}\right)\left(\mathbf{e}_{1} \cdot \mathbf{E}_{3}\right)+\left(\mathbf{e}_{2} \cdot \mathbf{E}_{1}\right)\left(\mathbf{e}_{2} \cdot \mathbf{E}_{3}\right)\right]\right.
$$$$
\left.-\left[X_{B}(z)-X_{G}\right]\left[\left(\mathbf{e}_{1} \cdot \mathbf{E}_{3}\right)^{2}+\left(\mathbf{e}_{2} \cdot \mathbf{E}_{3}\right)^{2}\right]\right\} \mathrm{d} z
$$

$$
\begin{aligned}
A_{36}^{[P]}=A_{63}^{[P]}=\Lambda \int_{0}^{L}\{ & {\left[X_{B}(z)-X_{G}\right]\left[\left(\mathbf{e}_{1} \cdot \mathbf{E}_{2}\right)\left(\mathbf{e}_{1} \cdot \mathbf{E}_{3}\right)+\left(\mathbf{e}_{2} \cdot \mathbf{E}_{2}\right)\left(\mathbf{e}_{2} \cdot \mathbf{E}_{3}\right)\right] } \\
& \left.-\left[Y_{B}(z)-Y_{G}\right]\left[\left(\mathbf{e}_{1} \cdot \mathbf{E}_{1}\right)\left(\mathbf{e}_{1} \cdot \mathbf{E}_{3}\right)+\left(\mathbf{e}_{2} \cdot \mathbf{E}_{1}\right)\left(\mathbf{e}_{2} \cdot \mathbf{E}_{3}\right)\right]\right\} \mathrm{d} z
\end{aligned}
$$

$$
\begin{aligned}
A_{45}^{[P]}=A_{54}^{[P]}=\Lambda \int_{0}^{L}\{- & {\left[X_{B}(z)-X_{G}\right]\left[Y_{B}(z)-Y_{G}\right]\left[\left(\mathbf{e}_{1} \cdot \mathbf{E}_{3}\right)^{2}+\left(\mathbf{e}_{2} \cdot \mathbf{E}_{3}\right)^{2}\right] } \\
& +\left[X_{B}(z)-X_{G}\right]\left[Z_{B}(z)-Z_{G}\right]\left[\left(\mathbf{e}_{1} \cdot \mathbf{E}_{2}\right)\left(\mathbf{e}_{1} \cdot \mathbf{E}_{3}\right)+\left(\mathbf{e}_{2} \cdot \mathbf{E}_{2}\right)\left(\mathbf{e}_{2} \cdot \mathbf{E}_{3}\right)\right] \\
& +\left[Y_{B}(z)-Y_{G}\right]\left[Z_{B}(z)-Z_{G}\right]\left[\left(\mathbf{e}_{1} \cdot \mathbf{E}_{1}\right)\left(\mathbf{e}_{1} \cdot \mathbf{E}_{3}\right)+\left(\mathbf{e}_{2} \cdot \mathbf{E}_{1}\right)\left(\mathbf{e}_{2} \cdot \mathbf{E}_{3}\right)\right] \\
& \left.-\left[Z_{B}(z)-Z_{G}\right]^{2}\left[\left(\mathbf{e}_{1} \cdot \mathbf{E}_{1}\right)\left(\mathbf{e}_{1} \cdot \mathbf{E}_{2}\right)+\left(\mathbf{e}_{2} \cdot \mathbf{E}_{1}\right)\left(\mathbf{e}_{2} \cdot \mathbf{E}_{2}\right)\right]\right\} \mathrm{d} z
\end{aligned}
$$

$$
\begin{aligned}
A_{46}^{[P]}=A_{64}^{[P]}=\Lambda \int_{0}^{L}\{ & {\left[X_{B}(z)-X_{G}\right]\left[Y_{B}(z)-Y_{G}\right]\left[\left(\mathbf{e}_{1} \cdot \mathbf{E}_{2}\right)\left(\mathbf{e}_{1} \cdot \mathbf{E}_{3}\right)+\left(\mathbf{e}_{2} \cdot \mathbf{E}_{2}\right)\left(\mathbf{e}_{2} \cdot \mathbf{E}_{3}\right)\right] } \\
& -\left[X_{B}(z)-X_{G}\right]\left[Z_{B}(z)-Z_{G}\right]\left[\left(\mathbf{e}_{1} \cdot \mathbf{E}_{2}\right)^{2}+\left(\mathbf{e}_{2} \cdot \mathbf{E}_{2}\right)^{2}\right] \\
& -\left[Y_{B}(z)-Y_{G}\right]^{2}\left[\left(\mathbf{e}_{1} \cdot \mathbf{E}_{1}\right)\left(\mathbf{e}_{1} \cdot \mathbf{E}_{3}\right)+\left(\mathbf{e}_{2} \cdot \mathbf{E}_{1}\right)\left(\mathbf{e}_{2} \cdot \mathbf{E}_{3}\right)\right] \\
& \left.+\left[Y_{B}(z)-Y_{G}\right]\left[Z_{B}(z)-Z_{G}\right]\left[\left(\mathbf{e}_{1} \cdot \mathbf{E}_{1}\right)\left(\mathbf{e}_{1} \cdot \mathbf{E}_{2}\right)+\left(\mathbf{e}_{2} \cdot \mathbf{E}_{1}\right)\left(\mathbf{e}_{1} \cdot \mathbf{E}_{2}\right)\right]\right\} \mathrm{d} z
\end{aligned}
$$




$$
\begin{aligned}
A_{56}^{[P]}=A_{65}^{[P]}=\Lambda \int_{0}^{L}\{ & {\left[X_{B}(z)-X_{G}\right]^{2}\left[\left(\mathbf{e}_{1} \cdot \mathbf{E}_{2}\right)\left(\mathbf{e}_{1} \cdot \mathbf{E}_{3}\right)+\left(\mathbf{e}_{2} \cdot \mathbf{E}_{2}\right)\left(\mathbf{e}_{1} \cdot \mathbf{E}_{3}\right)\right] } \\
& +\left[X_{B}(z)-X_{G}\right]\left[Y_{B}(z)-Y_{G}\right]\left[\left(\mathbf{e}_{1} \cdot \mathbf{E}_{1}\right)\left(\mathbf{e}_{1} \cdot \mathbf{E}_{3}\right)+\left(\mathbf{e}_{2} \cdot \mathbf{E}_{1}\right)\left(\mathbf{e}_{2} \cdot \mathbf{E}_{3}\right)\right] \\
& +\left[X_{B}(z)-X_{G}\right]\left[Z_{B}(z)-Z_{G}\right]\left[\left(\mathbf{e}_{1} \cdot \mathbf{E}_{1}\right)\left(\mathbf{e}_{1} \cdot \mathbf{E}_{2}\right)+\left(\mathbf{e}_{2} \cdot \mathbf{E}_{1}\right)\left(\mathbf{e}_{2} \cdot \mathbf{E}_{2}\right)\right] \\
& \left.-\left[Y_{B}(z)-Y_{G}\right]\left[Z_{B}(z)-Z_{G}\right]\left[\left(\mathbf{e}_{1} \cdot \mathbf{E}_{1}\right)^{2}+\left(\mathbf{e}_{2} \cdot \mathbf{E}_{1}\right)^{2}\right]\right\} \mathrm{d} z
\end{aligned}
$$

The procedure for the contribution of the extremities of the cylinder is the same, resulting in very similar expressions. For example the term $A_{56}^{[b]}$ is:

$$
\begin{aligned}
A_{56}^{[b]}=A_{65}^{[b]}=\rho C_{a V} V_{R} & \left\{-\left[X_{B}(0)-X_{G}\right]^{2}\left(\mathbf{e}_{3} \cdot \mathbf{E}_{2}\right)\left(\mathbf{e}_{3} \cdot \mathbf{E}_{3}\right)\right. \\
& +\left[X_{B}(0)-X_{G}\right]\left[Y_{B}(0)-Y_{G}\right]\left(\mathbf{e}_{3} \cdot \mathbf{E}_{1}\right)\left(\mathbf{e}_{3} \cdot \mathbf{E}_{3}\right) \\
& +\left[X_{B}(0)-X_{G}\right]\left[Z_{B}(0)-Z_{G}\right]\left(\mathbf{e}_{3} \cdot \mathbf{E}_{1}\right)\left(\mathbf{e}_{3} \cdot \mathbf{E}_{2}\right) \\
& \left.-\left[Y_{B}(0)-Y_{G}\right]\left[Z_{B}(0)-Z_{G}\right]\left(\mathbf{e}_{3} \cdot \mathbf{E}_{1}\right)^{2}\right\}
\end{aligned}
$$

and, as in Section 3.6, the contribution of an extremity of the cylinder is null if it is above the mean waterline.

The added mass matrix of the body is obtained by summing the contribution of all the cylinders that compose the structure, i.e. $\mathbb{A}=\sum_{j=1}^{N_{c}} \mathbb{A}_{j}$. 


\section{APPENDIX B - Analytical integration of the forces due to the local acceleration}

Following the slender-body approach presented in this text and using the same notation as Chapter 3, the forces due to the local acceleration of the fluid acting along the length of a cylinder are given by:

$$
\begin{aligned}
\mathbf{F}_{\phi^{(1)}}^{(1)} & =\int_{0}^{L_{0}} \rho \pi R^{2}\left(1+C_{a}\right)\left[\left(\frac{\partial \mathbf{u}^{(1)}}{\partial t} \cdot \mathbf{e}_{1}^{(0)}\right) \mathbf{e}_{1}^{(0)}+\left(\frac{\partial \mathbf{u}^{(1)}}{\partial t} \cdot \mathbf{e}_{2}^{(0)}\right) \mathbf{e}_{2}^{(0)}\right] \mathrm{d} z \\
\mathbf{F}_{\phi^{-}}^{(2)} & =\int_{0}^{L_{0}} \rho \pi R^{2}\left(1+C_{a}\right)\left[\left(\frac{\partial \mathbf{u}^{-}}{\partial t} \cdot \mathbf{e}_{1}^{(0)}\right) \mathbf{e}_{1}^{(0)}+\left(\frac{\partial \mathbf{u}^{-}}{\partial t} \cdot \mathbf{e}_{2}^{(0)}\right) \mathbf{e}_{2}^{(0)}\right] \mathrm{d} z
\end{aligned}
$$

with the origin of the local coordinate system of the cylinder, $O$, taken at its bottom. The moments with respect to the bottom of the cylinder are obtained by considering the lever $z$ in the expressions above.

The accelerations are obtained by differentiating the velocity potentials given by Equation 3.33 (first-order) and Equation 3.35 (difference-frequency second-order). To ease the expressions, they are rewritten in the following way:

$$
\begin{aligned}
\frac{\partial \mathbf{u}^{(1)}}{\partial t} & =\Re\left\{\sum_{j=1}^{N} \mathbf{a}_{j}^{(1)} e^{-i \omega_{j} t}\right\} \\
\frac{\partial \mathbf{u}^{-}}{\partial t} & =\Re\left\{\sum_{j=1}^{N} \sum_{l=1}^{N} \mathbf{a}_{j l}^{-} e^{-i\left(\omega_{j}-\omega_{l}\right) t}\right\}
\end{aligned}
$$

with

$$
\begin{aligned}
\mathbf{a}_{j}^{(1)} & =\mathcal{A}_{j}^{(1)}\left[\cosh \left(k_{j}(Z+h)\right) \mathbf{k}_{j}-i k_{j} \sinh \left(k_{j}(Z+h)\right) \mathbf{E}_{3}\right] e^{i \mathbf{k}_{j} \cdot \mathbf{x}} \\
\mathbf{a}_{j l}^{-} & =\mathcal{A}_{j l}^{-}\left[\cosh \left(\left\|\mathbf{k}_{j l}^{-}\right\|(Z+h)\right) \mathbf{k}_{j l}^{-}-i\left\|\mathbf{k}_{j l}^{-}\right\| \sinh \left(\left\|\mathbf{k}_{j l}^{-}\right\|(Z+h)\right) \mathbf{E}_{3}\right] e^{i \mathbf{k}_{j l}^{-} \cdot \mathbf{x}}
\end{aligned}
$$

and

$$
\begin{aligned}
\mathbf{k}_{j} & =k_{j} \cos \beta \mathbf{E}_{1}+k_{j} \sin \beta \mathbf{E}_{2} \\
\mathbf{k}_{j l}^{-} & =\left(k_{j} \cos \beta_{j}-k_{l} \cos \beta_{l}\right) \mathbf{E}_{1}+\left(k_{j} \sin \beta_{j}-k_{l} \sin \beta_{l}\right) \mathbf{E}_{2} \\
\mathcal{A}_{j}^{(1)} & =\frac{-i g A_{j}}{\cosh \left(k_{j} h\right)}
\end{aligned}
$$




$$
\mathcal{A}_{j l}^{-}=\frac{1}{2}\left(\gamma_{j l}^{-}+\gamma_{l j}^{-*}\right) \frac{\omega_{j}-\omega_{l}}{\cosh \left(\left\|\mathbf{k}_{j}-\mathbf{k}_{l}\right\| h\right)}
$$

where $\gamma_{j l}$ is given by Equation 3.37. Though it may be rather annoying to read the equations when written in such an indirect way, this notation makes it easier to integrate the fluid acceleration along the cylinder because the spatial dependency is concisely expressed in Equations B.5 and B.6. Besides, $\mathbf{a}_{j l}^{-}$is basically the same expression as $\mathbf{a}_{j}^{(1)}$, with $\mathbf{k}_{j l}^{-}$ replacing $\mathbf{k}_{j}$ and $\mathcal{A}_{j l}^{-}$instead of $\mathcal{A}_{j}^{(1)}$, in such a way that the results obtained for the integration of $\mathbf{a}_{j}^{(1)}$ can be readily extended for $\mathbf{a}_{j l}^{-}$. For this reason, the procedure below will be performed for $\mathbf{a}_{j}^{(1)}$ only.

The position of the cylinder is described by the location of the bottom and top extremities of its axis, denoted by $\left(X_{1}, Y_{1}, Z_{1}\right)$ and $\left(X_{2}, Y_{2}, Z_{2}\right)$. If the top extremity is above the mean waterline, the point with coordinates $\left(X_{2}, Y_{2}, Z_{2}\right)$ is taken as the intersection of the cylinder axis with the mean waterline, so that the wetted length of the cylinder is $L_{0}=\sqrt{\left(X_{2}-X_{1}\right)^{2}+\left(Z_{2}-Z_{1}\right)^{2}+\left(Z_{2}-Z_{1}\right)^{2}}$. The inclination of the cylinder axis with respect to the vertical is denoted by $\alpha$, while the angle between the projection of the cylinder with the plane $\mathrm{XY}$ with the $\mathrm{X}$ axis is denoted by $\psi$, thus yielding the following:

$$
\begin{array}{ll}
\mathbf{E}_{1} \cdot \mathbf{e}_{1}^{(0)}=\cos \theta \cos \psi & \mathbf{E}_{1} \cdot \mathbf{e}_{2}^{(0)}=-\cos \theta \sin \psi \\
\mathbf{E}_{2} \cdot \mathbf{e}_{1}^{(0)}=\cos \theta \sin \psi & \mathbf{E}_{2} \cdot \mathbf{e}_{2}^{(0)}=\cos \theta \cos \psi \\
\mathbf{E}_{3} \cdot \mathbf{e}_{1}^{(0)}=\sin \theta & \mathbf{E}_{3} \cdot \mathbf{e}_{2}^{(0)}=0
\end{array}
$$

and, finally:

$$
\begin{aligned}
& \mathbf{a}_{j}^{(1)} \cdot \mathbf{e}_{1}^{(0)}=\mathcal{A}_{j}^{(1)} k_{j}\left[\cosh \left(k_{j}(Z+h)\right) \cos \alpha \cos (\beta-\psi)-i \sinh \left(k_{j}(Z+h)\right) \sin \alpha\right] e^{i \mathbf{k}_{j} \cdot \mathbf{X}} \\
& \mathbf{a}_{j}^{(1)} \cdot \mathbf{e}_{2}^{(0)}=\mathcal{A}_{j}^{(1)} k_{j}\left[\cosh \left(k_{j}(Z+h)\right) \cos \alpha \sin (\beta-\psi)\right] e^{i \mathbf{k}_{j} \cdot \mathbf{X}}
\end{aligned}
$$

which are the parts of Equation B.1 that need to be integrated, with the first component acting along $\mathbf{e}_{1}^{(0)}$ and the second along $\mathbf{e}_{2}^{(0)}$. The integration is done considering three possible situations for the cylinder:

Horizontal cylinder $(\alpha=\pi / 2)$ that is perpendicular to the wave direction $(\boldsymbol{\psi}=\boldsymbol{\beta} \pm \pi / 2)$ : in this case, the fluid acceleration is constant along all the cylinder axis, yielding:

$$
\begin{aligned}
& \int_{0}^{L_{0}} \mathbf{a}_{j}^{(1)}(z) \cdot \mathbf{e}_{1}^{(0)} \mathrm{d} z=L_{0}\left(\mathbf{a}_{j}^{(1)}\left(z_{a}\right) \cdot \mathbf{e}_{1}^{(0)}\right) \\
& \int_{0}^{L_{0}} \mathbf{a}_{j}^{(1)}(z) \cdot \mathbf{e}_{2}^{(0)} \mathrm{d} z=L_{0}\left(\mathbf{a}_{j}^{(1)}\left(z_{a}\right) \cdot \mathbf{e}_{2}^{(0)}\right)
\end{aligned}
$$


with $z_{a}$ an arbitrary point on the cylinder axis. The moment with respect to the bottom node is obtained by multiplying the expressions above by $L_{0} / 2$.

Horizontal cylinder $(\alpha=\pi / 2)$ that is not perpendicular to the wave direction $(\boldsymbol{\psi} \neq \boldsymbol{\beta} \pm \boldsymbol{\pi} / \mathbf{2})$ : the position of a point on the cylinder axis, $\mathbf{X}_{B}$, is parameterized as follows:

$$
\begin{aligned}
& X_{B}(z)=X_{1}+z \frac{\left(X_{2}-X_{1}\right)}{L_{0}} \\
& Y_{B}(z)=Y_{1}+z \frac{\left(Y_{2}-Y_{1}\right)}{L_{0}}
\end{aligned}
$$

As the vertical coordinate $Z_{B}$ is constant, the depth dependent terms with cosh and sinh can be taken out of the integral, thus only $e^{i \mathbf{k}_{j} \cdot \mathbf{X}_{B}}$ needs to be integrated:

$$
\int_{0}^{L_{0}} e^{i\left[\mathbf{k}_{j} \cdot \mathbf{X}_{1}+\frac{z}{L_{0}} \mathbf{k}_{j} \cdot \Delta \mathbf{X}\right]} \mathrm{d} z=-\frac{i L_{0}\left(e^{i \mathbf{k}_{j} \cdot \mathbf{X}_{2}}-e^{i \mathbf{k}_{j} \cdot \mathbf{X}_{\mathbf{1}}}\right)}{\mathbf{k}_{j} \cdot \Delta \mathbf{X}}
$$

with $\Delta \mathbf{X}=\mathbf{X}_{2}-\mathbf{X}_{1}$. For the moments with respect to the bottom node, the integration of $z e^{i \mathbf{k}_{j} \cdot \mathbf{X}_{B}}$ is needed:

$$
\int_{0}^{L_{0}} z e^{i\left[\mathbf{k}_{j} \cdot \mathbf{X}_{1}+\frac{z}{L_{0}} \mathbf{k}_{j} \cdot \Delta \mathbf{X}\right]} \mathrm{d} z=\frac{L_{0}^{2}\left(e^{i \mathbf{k}_{j} \cdot \mathbf{X}_{\mathbf{2}}}-e^{i \mathbf{k}_{j} \cdot \mathbf{X}_{\mathbf{1}}}-i L_{0}^{2} \mathbf{k}_{j} \cdot \Delta \mathbf{X} e^{i \mathbf{k}_{j} \cdot \mathbf{X}_{2}}\right)}{\mathbf{k}_{j} \cdot \Delta \mathbf{X}}
$$

The results for the integration of $\mathbf{a}_{j}^{(1)}(z)$ can be readily obtained from the expressions above. For example, the integration of the y-component of the acceleration yields:

$$
\begin{aligned}
\int_{0}^{L_{0}} \mathbf{a}_{j}^{(1)}(z) \cdot \mathbf{e}_{2}^{(0)} \mathrm{d} z & =-i \mathcal{A}_{j}^{(1)} k_{j}\left[\cosh \left(k_{j}(Z+h)\right) \cos \alpha \sin (\beta-\psi)\right] \\
& \cdot \frac{L_{0}\left(e^{i \mathbf{k}_{j} \cdot \mathbf{X}_{2}}-e^{i \mathbf{k}_{j} \cdot \mathbf{X}_{1}}\right)}{\mathbf{k}_{j} \cdot \Delta \mathbf{X}} \\
\int_{0}^{L_{0}} \mathbf{a}_{j}^{(1)}(z) \cdot \mathbf{e}_{2}^{(0)} z \mathrm{~d} z & =-i \mathcal{A}_{j}^{(1)} k_{j}\left[\cosh \left(k_{j}(Z+h)\right) \cos \alpha \sin (\beta-\psi)\right] \\
& \cdot \frac{L_{0}^{2}\left(e^{i \mathbf{k}_{j} \cdot \mathbf{X}_{2}}-e^{i \mathbf{k}_{j} \cdot \mathbf{X}_{1}}-i L_{0}^{2} \mathbf{k}_{j} \cdot \Delta \mathbf{X} e^{i \mathbf{k}_{j} \cdot \mathbf{X}_{2}}\right)}{\mathbf{k}_{j} \cdot \Delta \mathbf{X}}
\end{aligned}
$$

Note that these expressions are not valid if $\mathbf{k}_{j} \cdot \Delta \mathbf{X}$, hence if the cylinder axis is parallel to the wave direction, which is the case dealt with above, or if $\mathbf{X}_{2}=\mathbf{X}_{1}$, in which case the cylinder is actually a plate.

Cylinder with inclination $\alpha \neq \pi / 2$ with respect to the vertical: in this case, the vertical coordinate of $\mathbf{X}_{B}$ is given by:

$$
Z_{B}=Z_{1}+\frac{z}{L_{0}} \underbrace{\left(Z_{2}-Z_{1}\right)}_{=\Delta Z}
$$


For the forces, the integral that needs to be solved is:

$$
\begin{aligned}
& \int_{0}^{L_{0}} \cosh \left[k_{j}\left(h+Z_{1}+\frac{z}{L} \Delta Z\right)\right] e^{i\left[\mathbf{k}_{j} \cdot \mathbf{X}_{1}+\frac{z}{L_{0}} \mathbf{k}_{j} \cdot \Delta \mathbf{X}\right]} \mathrm{d} z \\
& =\frac{L e^{i \mathbf{k}_{j} \cdot \mathbf{X}_{\mathbf{2}}}\left[i \mathbf{k}_{j} \cdot \Delta \mathbf{X} \cosh \left(k_{j}\left(Z_{2}+h\right)\right)-k_{j} \Delta Z \sinh \left(k_{j}\left(Z_{2}+h\right)\right)\right]}{\left[i \mathbf{k}_{j} \cdot \Delta \mathbf{X}-k_{j} \Delta Z\right]\left[i \mathbf{k}_{j} \cdot \Delta \mathbf{X}+k_{j} \Delta Z\right]} \\
& -\frac{L e^{i \mathbf{k}_{j} \cdot \mathbf{X}_{\mathbf{1}}}\left[i \mathbf{k}_{j} \cdot \Delta \mathbf{X} \cosh \left(k_{j}\left(Z_{1}+h\right)\right)-k_{j} \Delta Z \sinh \left(k_{j}\left(Z_{1}+h\right)\right)\right]}{\left[i \mathbf{k}_{j} \cdot \Delta \mathbf{X}-k_{j} \Delta Z\right]\left[i \mathbf{k}_{j} \cdot \Delta \mathbf{X}+k_{j} \Delta Z\right]}
\end{aligned}
$$

while the following is required to evaluate the moments:

$$
\begin{aligned}
& \int_{0}^{L_{0}} \cosh \left[k_{j}\left(h+Z_{1}+\frac{z}{L} \Delta Z\right)\right] e^{i\left[\mathbf{k}_{j} \cdot \mathbf{X}_{1}+\frac{z}{L_{0}} \mathbf{k}_{j} \cdot \Delta \mathbf{X}\right]} z \mathrm{~d} z \\
& \quad=\frac{L^{2} e^{i \mathbf{k}_{j} \mathbf{X}_{2}} k_{j} \Delta Z\left[\left(\mathbf{k}_{j} \cdot \Delta \mathbf{X}\right)^{2}+2 \mathbf{k}_{j} \cdot \Delta \mathbf{X}+\left(k_{j} \Delta Z\right)^{2}\right] \sinh \left(k_{j}\left(Z_{2}+h\right)\right)}{\left[i \mathbf{k}_{j} \cdot \Delta \mathbf{X}-k_{j} \Delta Z\right]^{2}\left[i \mathbf{k}_{j} \cdot \Delta \mathbf{X}+k_{j} \Delta Z\right]^{2}} \\
& +\frac{L^{2} e^{i \mathbf{k}_{j} \mathbf{X}_{2}}\left[-i\left(\mathbf{k}_{j} \cdot \Delta \mathbf{X}\right)^{3}+\left(\mathbf{k}_{j} \Delta \mathbf{X}\right)^{2}-i \mathbf{k}_{j} \cdot \Delta \mathbf{X} k_{j} \Delta Z-\left(k_{j} \Delta Z\right)^{2}\right] \cosh \left(k_{j}\left(Z_{2}+h\right)\right)}{\left[i \mathbf{k}_{j} \cdot \Delta \mathbf{X}-k_{j} \Delta Z\right]^{2}\left[i \mathbf{k}_{j} \Delta \mathbf{X}+k_{j} \Delta Z\right]^{2}} \\
& -\frac{L^{2} e^{i \mathbf{k}_{j} \mathbf{X}_{1}}\left\{2 k_{j} \Delta Z \mathbf{k}_{j} \cdot \Delta \mathbf{X} \sinh \left(k_{j}\left(Z_{1}+h\right)\right)+\left[\left(\mathbf{k}_{j} \cdot \Delta \mathbf{X}\right)^{2}-\left(k_{j} \Delta Z\right)^{2}\right] \cosh \left(k\left(Z_{1}+h\right)\right)\right\}}{\left[i \mathbf{k}_{j} \cdot \Delta \mathbf{X}-k_{j} \Delta Z\right]^{2}\left[i \mathbf{k}_{j} \cdot \Delta \mathbf{X}+k_{j} \Delta Z\right]^{2}}
\end{aligned}
$$

The forces/moments for the contribution of the vertical acceleration, which has a dependency with respect to $Z_{B}$ in sinh instead of cosh, are simply obtained by replacing cosh by sinh and sinh by cosh in the expressions above.

With the solution of these integrals, the results for the integration of $\mathbf{a}_{j}^{(1)}(z)$ can be readily obtained, as illustrated in the previous case of the horizontal cylinder. 


\section{APPENDIX C - Example of a METiS input file}

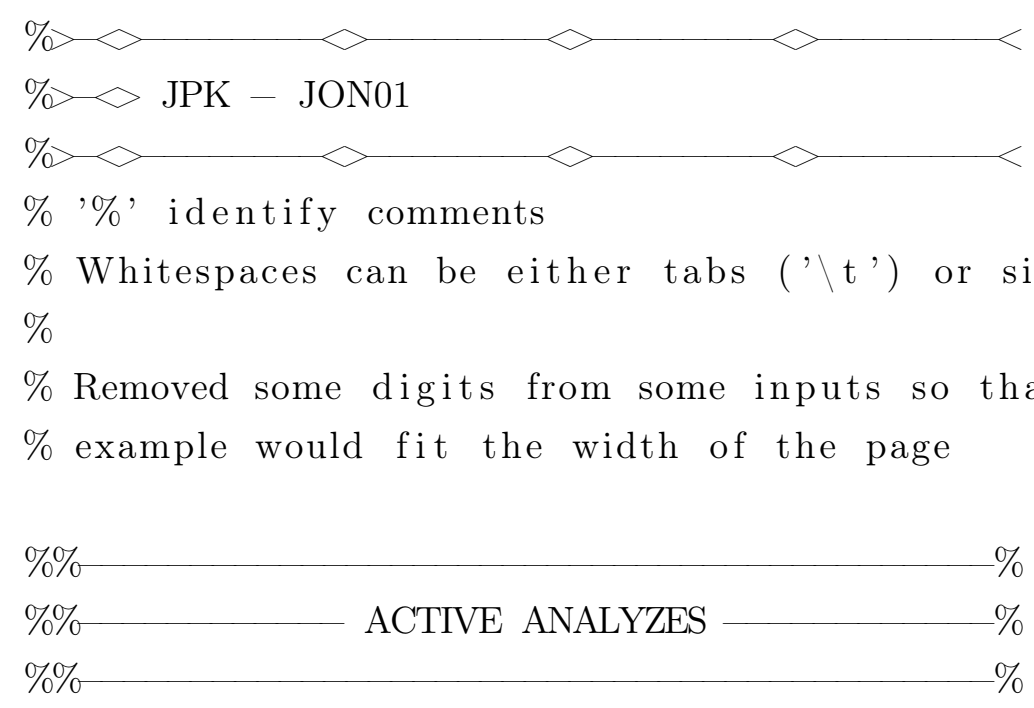

Hydro $2 \%$ 0: Disabled; 1: First-order; 2: Second-order

Aero $\quad 0 \%$ : Disabled; 1 : Use BEMT

Moor $\quad 1 \%$ 0: Disabled; 1: Linear mooring model

$\%$ For each of the 6 degrees of freedom

$\% \rightarrow 0$ : Disable selected dof in the equations of motion,

$\%$ but loads are still evaluated

$\% \rightarrow 1:$ Enable selected dof in the equations of motion

DOFS $1 \begin{array}{llllll}1 & 1 & 1 & 1 & 1 & 1\end{array}$

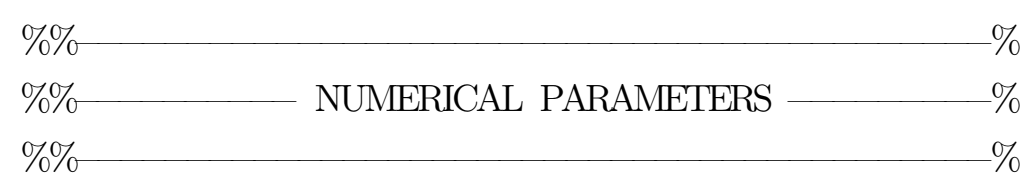
TimeStep $\quad 0.0894 \%$ Time step, in seconds
PrintStep $\quad 0.0894 \%$ Print step, in seconds
TimeTotal $\quad 11000 \%$ Total simulation time, in seconds
TimeRamp $\quad 0 \quad \%$ Numerical ramp, in seconds

$\%$ If HydroMode $!=2$ and WaveStret $=1$, the latter is ignored

$\%$ 0: No stretching

$\%$ 1: Vertical stretching (same as from Kim and Chen, 1994)

$\%$ 2: Wheeler's stretching (under development)

WaveStret 1 


\begin{tabular}{|c|c|c|}
\hline & \multirow{2}{*}{ ENVIRONMENT $\% \%$} \\
\hline & & \\
\hline rav & 9.81 & $\%$ Acceleration of gravity \\
\hline JatDens & 998.2 & $\%$ Water density \\
\hline AirDens & 1.225 & $\%$ Air density \\
\hline WatDepth & 302.8 & $\%$ Water depth (constant) \\
\hline WindVel & 0 & $\%$ Measured at WindHeight \\
\hline adDir & 180 & $\%$ Wind direction \\
\hline WindHeight & 90 & $\%$ Reference height \\
\hline dexp & 0.2 & $\%$ Exponent for wind profile \\
\hline UseTipLoss & 1 & $\%$ 1: Use tip loss factor \\
\hline eHubLoss & 1 & $\%$ 1: Use hub loss factor \\
\hline
\end{tabular}

Wave

$\%$ Available options:

$\%$ Input wave components individually

$\% \rightarrow$ WRWAVE $\backslash \mathrm{t}$ Height $\backslash \mathrm{t}$ Ang. freq. $\backslash \mathrm{t}$ Direction (deg) $\backslash \mathrm{t}$ Phase (deg)

$\%$ FRWAVE $\backslash \mathrm{t}$ Height $\backslash \mathrm{t}$ Freq. $\backslash \mathrm{t}$ Direction (deg) $\backslash \mathrm{t}$ Phase (deg)

$\%$ TRWAVE $\backslash \mathrm{t}$ Height $\backslash \mathrm{t}$ Period $\backslash \mathrm{t}$ Direction (deg) $\backslash \mathrm{t}$ Phase (deg)

$\%$

$\%$ Specify a JONSWAP spectrum

$\% \rightarrow$ JONSW $\backslash \mathrm{t}$ Hs $\backslash \mathrm{t}$ Tp $\backslash \mathrm{t}$ Gamma $\backslash \mathrm{t}$ Direction $(\mathrm{deg}) \backslash \mathrm{t}$ wlow $(\mathrm{rad} / \mathrm{s}) \ldots$

$\% \backslash \mathrm{t}$ whigh $(\mathrm{rad} / \mathrm{s}) \backslash \mathrm{t}$ Seed for RNG

$\%$ The seed value can be any double or the char '?', which

$\%$ specifiesthat the seed should be set by the simulation

$\%$ (hence, each simulation would result in a different sea)

$\%$

$\% \rightarrow$ Input a series of wave elevation measured at $(0,0,0)$

$\%$ Elev $\backslash \mathrm{t}$ Path, Direction, wlow, whigh

$\%$ 'Path' is the path to a text file with two columns, with

$\%$ the first being the time vector and the second the wave

$\%$ elevation measured at $(0,0,0)$

$\%$

Elev G: $\backslash$ Meu Drive $\backslash$ Doutorado $\backslash 1$ Testes_Jappaku $\backslash$ IRR10.Elev, $180,0.03,1.45$ $\mathrm{END}$

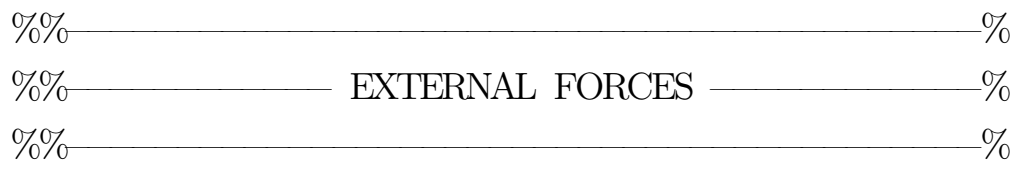

$\%$ External constant forces and moments

ExtConstForce $0,0,-3.30110 \mathrm{e}+06,0,0,0$ 
\% External linear stiffness matrix

ExtLinStiff
$6.92 \mathrm{e}+04$
$0.00 \mathrm{e}+00$
$0.00 \mathrm{e}+00 \quad 0.00 \mathrm{e}+00$
$5.37 \mathrm{e}+05$
$0.00 \mathrm{e}+00$
$0.00 \mathrm{e}+00$
$6.92 \mathrm{e}+04$
$0.00 \mathrm{e}+00-5.37 \mathrm{e}+05$
$0.00 \mathrm{e}+00$
$0.00 \mathrm{e}+00$
$0.00 \mathrm{e}+00$
$0.00 \mathrm{e}+00$
$1.04 \mathrm{e}+05 \quad 0.00 \mathrm{e}+00$
$0.00 \mathrm{e}+00$
$0.00 \mathrm{e}+00$
$0.00 \mathrm{e}+00-5.37 \mathrm{e}+05$
$0.00 \mathrm{e}+00$
$1.07 \mathrm{e}+08$
$0.00 \mathrm{e}+00$
$0.00 \mathrm{e}+00$
$5.37 \mathrm{e}+05$
$0.00 \mathrm{e}+00$
$0.00 \mathrm{e}+00$
$0.00 \mathrm{e}+00$
$1.07 \mathrm{e}+08$
$0.00 \mathrm{e}+00$
$0.00 \mathrm{e}+00$
$0.00 \mathrm{e}+00$
$0.00 \mathrm{e}+00 \quad 0.00 \mathrm{e}+00$
$0.00 \mathrm{e}+00$
$9.12 \mathrm{e}+07$

\% External linear damping matrix

ExtLinDamp
$\begin{array}{llllll}0.00 \mathrm{e}+00 & 0.00 \mathrm{e}+00 & 0.00 \mathrm{e}+00 & 0.00 \mathrm{e}+00 & 0.00 \mathrm{e}+00 & 0.00 \mathrm{e}+00\end{array}$
$\begin{array}{llllll}0.00 \mathrm{e}+00 & 0.00 \mathrm{e}+00 & 0.00 \mathrm{e}+00 & 0.00 \mathrm{e}+00 & 0.00 \mathrm{e}+00 & 0.00 \mathrm{e}+00\end{array}$
$\begin{array}{llllll}0.00 \mathrm{e}+00 & 0.00 \mathrm{e}+00 & 1.85 \mathrm{e}+05 & 0.00 \mathrm{e}+00 & 0.00 \mathrm{e}+00 & 0.00 \mathrm{e}+00\end{array}$
$\begin{array}{llllll}0.00 \mathrm{e}+00 & 0.00 \mathrm{e}+00 & 0.00 \mathrm{e}+00 & 2.66 \mathrm{e}+03 & 0.00 \mathrm{e}+00 & 0.00 \mathrm{e}+00\end{array}$
$\begin{array}{llllll}0.00 \mathrm{e}+00 & 0.00 \mathrm{e}+00 & 0.00 \mathrm{e}+00 & 0.00 \mathrm{e}+00 & 2.66 \mathrm{e}+03 & 0.00 \mathrm{e}+00\end{array}$
$\begin{array}{llllll}0.00 \mathrm{e}+00 & 0.00 \mathrm{e}+00 & 0.00 \mathrm{e}+00 & 0.00 \mathrm{e}+00 & 0.00 \mathrm{e}+00 & 0.00 \mathrm{e}+00\end{array}$

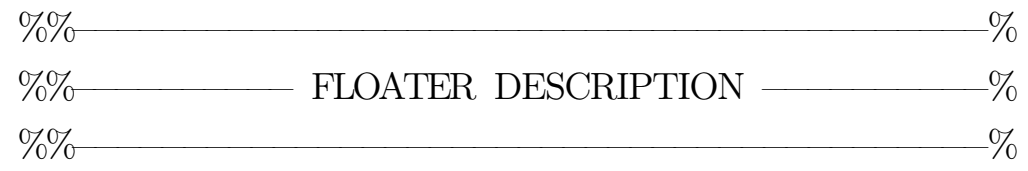

$\%$ At present, these must be the total inertia of the system, $\%$ but in the future it should be the inertia of the floater only FloaterMass 7.002 e6 \% Mass

FloaterCoG $0,0,-4.40 \%$ Coordinates of the center of gravity

$\%$ Terms of the inertia matrix

$\%$ Ixx, Iyy, Izz, Ixy, Ixz, Iyz

FloaterInertia $3.3 \mathrm{E}+09,3.3 \mathrm{E}+09,2.9 \mathrm{E}+09,6.0 \mathrm{E}+06,-1.9 \mathrm{E}+07,1.5 \mathrm{E}+06$

MeanDisp $0,0,0,0,0,0 \%$ Mean displacement to compute hydrodynamics

Disp0 $0,0,0,0,0,0 \%$ Initial displacement

Vel0 $\quad 0,0,0,0,0,0 \%$ Initial velocity

$\%=$ Nodes used to identify the cylinders

$\%=$ Node ID, X coord, Y coord, Z coord

$\%=$ IDs must be unique and in ascending order

Nodes

$\%$ Main column

$1,0,0,-20$

$2,0,0,10$ 
$\%$

$\%$ Stern column

$3,-25.40,0,-20$

$4,-25.40,0,10$

$\%$

$\%$ Bow column - Port

$5,12.70,22.00,-20$

$6,12.70,22.00,10$

$\%$

$\%$ Bow column - Starboard

$7,12.70,-22.00,-20$

$8,12.70,-22.00,10$

$\mathrm{END}$

\% Characteristic of the Morison Elements

Morison_circ

$\%$ 1st node ID $\backslash \mathrm{t}$ 2nd node ID $\backslash \mathrm{t}$ Diameter $\backslash \mathrm{t}$ C_Dx $\backslash \mathrm{t}$ C_Mx $\backslash \mathrm{t} \ldots$

$\%$ Number of integration points $\backslash \mathrm{t}$ C_dz node $1 \backslash \mathrm{t} \mathrm{C}_{-}$az node $1 \backslash \mathrm{t} \ldots$

\% C_dz node $2 \backslash \mathrm{t}$ C_az node $2 \backslash \mathrm{t}$ 1: Calculate FK pressure at ends

$\begin{array}{lllllllllll}1 & 2 & 15.0 & 1.50 & 1.82 & 50 & 3.0 & 0.68 & 0 & 0 & 1 \\ 3 & 4 & 9.0 & 1.50 & 1.82 & 50 & 3.0 & 0.68 & 0 & 0 & 1 \\ 5 & 6 & 9.0 & 1.50 & 1.82 & 50 & 3.0 & 0.68 & 0 & 0 & 1 \\ 7 & 8 & 9.0 & 1.50 & 1.82 & 50 & 3.0 & 0.68 & 0 & 0 & 1\end{array}$

END

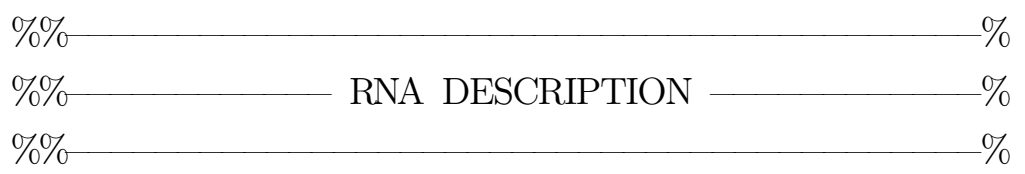

RotSpeed $0 \quad \%$ Rotor speed in rpm (constant)

RotTilt $0 \%$ Rotor tilt angle (degrees)

RotYaw $180 \%$ Rotor yaw angle (degrees)

NumBlades $3 \%$ Number of blades

BldPitch $0 \quad \%$ Blade pitch angle (degrees)

BldPrecone $-2.5 \%$ Blade coning angle (degrees)

HubRadius $\quad 1.5 \quad \%$ Hub radius

HubHeight $\quad 81.48 \%$ Hub height from $Z=0$

Overhang $\quad-5$ 


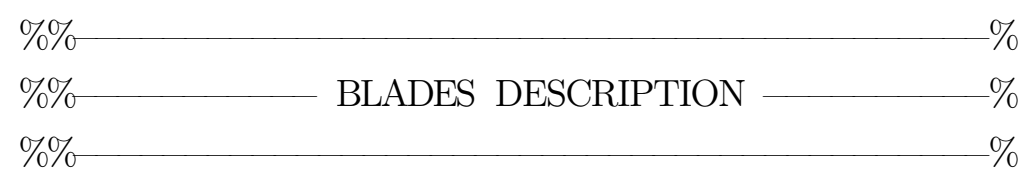

$\%$ Aerodynamic properties of the blades

$\%$ Parameteres are the same as OpenFAST

Blades_aero

\% BlSpn BlCrvAC BlSwpAC BlCrvAng BlTwist BlChord BlAFID

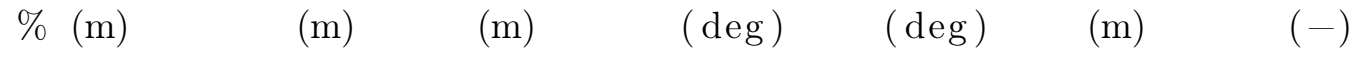

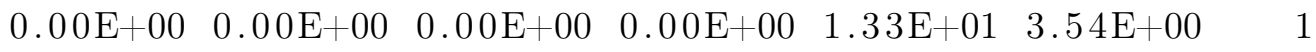

$\begin{array}{lllllll}1.36 \mathrm{E}+00 & 0.00 \mathrm{E}+00 & 0.00 \mathrm{E}+00 & 0.00 \mathrm{E}+00 & 1.33 \mathrm{E}+01 & 3.54 \mathrm{E}+00 & 1\end{array}$

$\begin{array}{lllllll}4.10 \mathrm{E}+00 & 0.00 \mathrm{E}+00 & 0.00 \mathrm{E}+00 & 0.00 \mathrm{E}+00 & 1.33 \mathrm{E}+01 & 3.85 \mathrm{E}+00 & 1\end{array}$

$\begin{array}{lllllll}6.83 \mathrm{E}+00 & 0.00 \mathrm{E}+00 & 0.00 \mathrm{E}+00 & 0.00 \mathrm{E}+00 & 1.33 \mathrm{E}+01 & 4.16 \mathrm{E}+00 & 2\end{array}$

$\begin{array}{lllllll}1.02 \mathrm{E}+01 & 0.00 \mathrm{E}+00 & 0.00 \mathrm{E}+00 & 0.00 \mathrm{E}+00 & 1.33 \mathrm{E}+01 & 4.55 \mathrm{E}+00 & 3\end{array}$

$\begin{array}{lllllll}1.43 \mathrm{E}+01 & 0.00 \mathrm{E}+00 & 0.00 \mathrm{E}+00 & 0.00 \mathrm{E}+00 & 1.14 \mathrm{E}+01 & 4.65 \mathrm{E}+00 & 4\end{array}$

$\begin{array}{lllllll}1.84 \mathrm{E}+01 & 0.00 \mathrm{E}+00 & 0.00 \mathrm{E}+00 & 0.00 \mathrm{E}+00 & 1.01 \mathrm{E}+01 & 4.45 \mathrm{E}+00 & 4\end{array}$

$\begin{array}{llllllll}2.25 \mathrm{E}+01 & 0.00 \mathrm{E}+00 & 0.00 \mathrm{E}+00 & 0.00 \mathrm{E}+00 & 9.01 \mathrm{E}+00 & 4.24 \mathrm{E}+00 & 5\end{array}$

$\begin{array}{lllllll}2.66 \mathrm{E}+01 & 0.00 \mathrm{E}+00 & 0.00 \mathrm{E}+00 & 0.00 \mathrm{E}+00 & 7.79 \mathrm{E}+00 & 4.00 \mathrm{E}+00 & 6\end{array}$

$\begin{array}{lllllll}3.07 \mathrm{E}+01 & 0.00 \mathrm{E}+00 & 0.00 \mathrm{E}+00 & 0.00 \mathrm{E}+00 & 6.54 \mathrm{E}+00 & 3.74 \mathrm{E}+00 & 6\end{array}$

$\begin{array}{lllllll}3.48 \mathrm{E}+01 & 0.00 \mathrm{E}+00 & 0.00 \mathrm{E}+00 & 0.00 \mathrm{E}+00 & 5.36 \mathrm{E}+00 & 3.50 \mathrm{E}+00 & 7\end{array}$

$\begin{array}{lllllll}3.89 \mathrm{E}+01 & 0.00 \mathrm{E}+00 & 0.00 \mathrm{E}+00 & 0.00 \mathrm{E}+00 & 4.18 \mathrm{E}+00 & 3.25 \mathrm{E}+00 & 7\end{array}$

$\begin{array}{lllllll}4.30 \mathrm{E}+01 & 0.00 \mathrm{E}+00 & 0.00 \mathrm{E}+00 & 0.00 \mathrm{E}+00 & 3.12 \mathrm{E}+00 & 3.01 \mathrm{E}+00 & 8\end{array}$

$\begin{array}{lllllll}4.71 \mathrm{E}+01 & 0.00 \mathrm{E}+00 & 0.00 \mathrm{E}+00 & 0.00 \mathrm{E}+00 & 2.31 \mathrm{E}+00 & 2.76 \mathrm{E}+00 & 8\end{array}$

$\begin{array}{lllllll}5.12 \mathrm{E}+01 & 0.00 \mathrm{E}+00 & 0.00 \mathrm{E}+00 & 0.00 \mathrm{E}+00 & 1.52 \mathrm{E}+00 & 2.51 \mathrm{E}+00 & 8\end{array}$

$\begin{array}{lllllll}5.46 \mathrm{E}+01 & 0.00 \mathrm{E}+00 & 0.00 \mathrm{E}+00 & 0.00 \mathrm{E}+00 & 8.63 \mathrm{E}-01 & 2.31 \mathrm{E}+00 & 8\end{array}$

$\begin{array}{lllllll}5.74 \mathrm{E}+01 & 0.00 \mathrm{E}+00 & 0.00 \mathrm{E}+00 & 0.00 \mathrm{E}+00 & 3.70 \mathrm{E}-01 & 2.08 \mathrm{E}+00 & 8\end{array}$

$\begin{array}{lllllll}6.01 \mathrm{E}+01 & 0.00 \mathrm{E}+00 & 0.00 \mathrm{E}+00 & 0.00 \mathrm{E}+00 & 1.06 \mathrm{E}-01 & 1.41 \mathrm{E}+00 & 8\end{array}$

$\begin{array}{lllllll}6.15 \mathrm{E}+01 & 0.00 \mathrm{E}+00 & 0.00 \mathrm{E}+00 & 0.00 \mathrm{E}+00 & 1.06 \mathrm{E}-01 & 1.41 \mathrm{E}+00 & 8\end{array}$

$\mathrm{END}$

$\%$ Airfoils that describe the blades

Airfoil_data \% Data from 'Cylinder1.dat'

\begin{tabular}{|c|c|c|c|}
\hline Alpha & $\mathrm{Cl}$ & $\mathrm{Cd}$ & $\mathrm{Cm}$ \\
\hline$(\operatorname{deg})$ & $(-)$ & $(-)$ & $(-)$ \\
\hline-180.00 & 0.000 & 0.5000 & 0.0 \\
\hline 0.00 & 0.000 & 0.5000 & 0.0 \\
\hline 180.00 & 0.000 & 0.5000 & 0.0 \\
\hline
\end{tabular}

END

Airfoil_data \% Data from 'Cylinder2.dat'

$\begin{array}{lllll}\% & \text { Alpha } & \mathrm{Cl} & \mathrm{Cd} & \mathrm{Cm} \\ \% & (\text { deg }) & (-) & (-) & (-)\end{array}$




$\begin{array}{rrrr}-180.00 & 0.000 & 0.3500 & 0.0 \\ 0.00 & 0.000 & 0.3500 & 0.0 \\ 180.00 & 0.000 & 0.3500 & 0.0\end{array}$

END

\% The specification of the other airfoils

$\%$ spans more than 1000 lines, so it is omitted

$\%$ Output channels

OUTPUT

\begin{tabular}{|c|c|}
\hline fowt_disp & $\%$ Displacement \\
\hline fowt_acc & $\%$ Acceleration \\
\hline hs_force & $\%$ Hydrostatic forces \\
\hline moor_force & $\%$ Mooring forces \\
\hline hd_force & $\%$ Total hydrodynamic forces \\
\hline hd_drag_force & $\%$ Hydrodynamic drag forces \\
\hline hd_force_1stP & $\%$ Hydrodynamic force due to 1 st ord potential \\
\hline hd_force_2ndP & $\%$ Hydrodynamic force due to 2 nd ord potential \\
\hline hd_force_conv & $\%$ Hydrodynamic force due to convective acc \\
\hline hd_force_acgr & $\%$ Hydrodynamic force due to acc/pressure grad \\
\hline hd_force_rslb & $\%$ Hydrodynamic force due rotation (slender body) \\
\hline hd_force_rotn & $\%$ Hydrodynamic force due to rotation of the normal \\
\hline hd_force_axdv & $\%$ Hydrodynamic force due to the axial-div acc \\
\hline hd_force_eta & \% Hydrodynamic force due to the relative wave elev \\
\hline hd_force_rem & \% Some of Rainey's point loads + centripetal acc \\
\hline $\begin{array}{l}\text { ad_add_mass_f } \\
\text { total_force }\end{array}$ & $\begin{array}{l}\text { force } \% \text { Force due to the variation of the added mass } \\
\% \text { Total force }\end{array}$ \\
\hline vave_elev 1 & $\%$ Wave elevation at specified node \\
\hline
\end{tabular}

END 


\section{APPENDIX D - Semi-analytic solution for the difference-frequency second-order wave}

\section{forces on a bottom-mounted vertical cylinder}

Continuing from Section 5.1.1.1, the incident part of the horizontal forces due to the first-order potential are given by:

$$
\begin{aligned}
f_{I, \nabla^{2}, j l}^{-} & =-\frac{\rho}{4} \int_{-h}^{0} \int_{0}^{2 \pi}\left(\nabla \varphi_{j}^{(1)} \cdot \nabla \varphi_{l}^{(1) *}\right)_{r=R} R \cos \theta \mathrm{d} \theta \mathrm{d} z \\
f_{I, \eta, j l}^{-} & =\frac{\rho \omega_{j} \omega_{l}}{4 g} \int_{-\pi}^{\pi}\left(\varphi_{j}^{(1)} \varphi_{l}^{(1) *}\right)_{\substack{r=R \\
z=0}} R \cos \theta \mathrm{d} \theta
\end{aligned}
$$

with $\varphi_{j}^{(1)}$ the complex amplitude of the first-order incident wave potential for unitary wave amplitude, i.e. $\varphi_{j}^{(1)}=\Re\left\{A_{j} \varphi_{j}^{(1)} e^{-i \omega_{j} t}\right\}$. Though its expression was already provided in Section 3.2, it is better to rewrite $\varphi_{j}^{(1)}$ using cylindrical coordinates, which are more suited to this problem. The expression can be found in MacCamy and Fuchs (1954), as reproduced below:

$$
\varepsilon \varphi_{j}^{(1)}=-\frac{i g}{\omega_{j}} \frac{\cosh \left(k_{j}(z+h)\right)}{\cosh \left(k_{j} h\right)} \sum_{n=0}^{\infty} \sigma_{n} i^{n} J_{n}\left(k_{j} r\right) \cos (n \theta)
$$

where $J_{n}$ is the Bessel function of the first kind of order $n$ and $\sigma_{n}=1$ for $n=0$ and $\sigma_{n}=2$ otherwise.

Substitution of $\varphi_{j}^{(1)}$ in the expressions above lead to the following nondimensional horizontal forces acting on the cylinder due to the incoming first-order wave potential:

$$
\begin{aligned}
\bar{f}_{I, \nabla^{2}, j l}^{-} & =\frac{f_{I, \nabla^{2}, j l}^{-}}{\rho g R}=i \pi \sqrt{\frac{\bar{k}_{j} \bar{h}}{\tanh \left(\bar{k}_{j} \bar{h}\right)}} \sqrt{\frac{\bar{k}_{l} \bar{h}}{\tanh \left(\bar{k}_{l} \bar{h}\right)}} \sum_{n=0}^{\infty} \frac{J_{n+1}\left(\bar{k}_{j}\right) J_{n}\left(\bar{k}_{l}\right)-J_{n}\left(\bar{k}_{j}\right) J_{n+1}\left(\bar{k}_{l}\right)}{\cosh \left(\bar{k}_{j} \bar{h}\right) \cosh \left(\bar{k}_{l} \bar{h}\right)} \\
\cdot & {\left[I_{j l}^{-}+I_{j l}^{+}\left(\frac{n(n+1)}{\bar{k}_{j} \bar{k}_{l}}+\frac{J_{n+1}^{\prime}\left(\bar{k}_{j}\right) J_{n}^{\prime}\left(\bar{k}_{l}\right)-J_{n}^{\prime}\left(\bar{k}_{j}\right) J_{n+1}^{\prime}\left(\bar{k}_{l}\right)}{J_{n+1}\left(\bar{k}_{j}\right) J_{n}\left(\bar{k}_{l}\right)-J_{n}\left(\bar{k}_{j}\right) J_{n+1}\left(\bar{k}_{l}\right)}\right)\right] } \\
\bar{f}_{I, \eta^{2}, j l}^{-}= & \frac{f_{I, \eta, j l}^{-}}{\rho g R}=-\frac{i \pi}{2} \sum_{n=0}^{\infty} J_{n+1}\left(\bar{k}_{j}\right) J_{n}\left(\bar{k}_{l}\right)-J_{n}\left(\bar{k}_{j}\right) J_{n+1}\left(\bar{k}_{l}\right)
\end{aligned}
$$


APPENDIX D. Semi-analytic solution for the difference-frequency second-order wave forces on a 202 bottom-mounted vertical cylinder

with the prime denoting the derivative and the auxiliary variables as follows:

$$
\begin{aligned}
I_{j l}^{ \pm} & =\frac{1}{2}\left[\frac{\sinh \left(\bar{k}_{j l}^{+} \bar{h}\right)}{\bar{k}_{j l}^{+} \bar{h}} \pm \frac{\sinh \left(\bar{k}_{j l}^{-} \bar{h}\right)}{\bar{k}_{j l}^{-\bar{h}}}\right] \\
\bar{k}_{j l}^{ \pm} & =\bar{k}_{j} \pm \bar{k}_{l}
\end{aligned}
$$

To evaluate the perturbation part of the horizontal forces due to the first-order potential, it is easier to calculate the corresponding force due to the total first-order potential, $\Phi_{j}^{(1)}=\varphi_{j}^{(1)}+\tilde{\varphi}_{j}^{(1)}$, and then subtract the contribution of the incident part:

$$
\begin{aligned}
f_{P, \nabla^{2}, j l}^{-} & =f_{\nabla^{2}, j l}^{-}-f_{I, \nabla^{2}, j l}^{-} \\
f_{P, \eta, j l}^{-} & =f_{\eta, j l}^{-}-f_{I, \eta, j l}^{-}
\end{aligned}
$$

with

$$
\begin{aligned}
f_{\nabla^{2}, j l}^{-} & =-\frac{\rho}{4} \int_{-h}^{0} \int_{0}^{2 \pi}\left(\nabla \Phi_{j}^{(1)} \cdot \nabla \Phi_{l}^{(1) *}\right)_{r=R} R \cos \theta \mathrm{d} \theta \mathrm{d} Z \\
f_{\eta, j l}^{-} & =\frac{\rho \omega_{j} \omega_{l}}{4 g} \int_{-\pi}^{\pi}\left(\Phi_{j}^{(1)} \Phi_{l}^{(1) *}\right)_{\substack{r=R \\
Z=0}} R \cos \theta \mathrm{d} \theta
\end{aligned}
$$

The first-order perturbation potential for unitary wave amplitude, required to compute the total first-order wave potential and also presented by MacCamy and Fuchs (1954), is given by:

$$
\tilde{\varphi}_{j}^{(1)}=\frac{i g}{\omega_{j}} \frac{\cosh \left(k_{j}(Z+h)\right)}{\cosh \left(k_{j} h\right)} \sum_{n=0}^{\infty} \sigma_{n} i^{n} \frac{J_{n}\left(k_{j} R\right)}{H_{n}^{\prime}\left(k_{j} R\right)} J_{n}\left(k_{j} r\right) \cos (n \theta)
$$

where $H_{n}$ is the Hankel function of the first kind of order $n$. The expressions above yield:

$$
\begin{aligned}
\bar{f}_{\nabla^{2}, j l}^{-} & =\frac{2 i}{\pi} \sqrt{\frac{\bar{h}}{\bar{k}_{j} \tanh \left(\bar{k}_{j} \bar{h}\right)}} \sqrt{\frac{\bar{h}}{\bar{k}_{l} \tanh \left(\bar{k}_{l} \bar{h}\right)} \sum_{n=0}^{\infty} \frac{\Omega_{n, j l}^{-}\left[I_{j l}^{-}+I_{j l}^{+} n(n+1) /\left(\bar{k}_{j} \bar{k}_{l}\right)\right]}{\cosh \left(\bar{k}_{j} \bar{h}\right) \cosh \left(\bar{k}_{l} \bar{h}\right)}} \\
\bar{f}_{\eta, j l}^{-} & =-\frac{2 i}{\pi \bar{k}_{j} \bar{k}_{j}} \sum_{n=0}^{\infty} \Omega_{n, j l}^{-}
\end{aligned}
$$

with

$$
\Omega_{n, j l}^{-}=\frac{1}{H_{n+1}^{\prime}\left(\bar{k}_{j}\right) H_{n}^{\prime *}\left(\bar{k}_{l}\right)}-\frac{1}{H_{n}^{\prime}\left(\bar{k}_{j}\right) H_{n+1}^{\prime *}\left(\bar{k}_{l}\right)}
$$

The sum of Equations D.13 and D.14 is equal to Equation A3 from Kim and Yue (1990), where the different components due to the incident and the perturbation potentials are not given separately. However, this distinction is made regarding the contribution 
of the second-order wave potential, hence the expressions provided therein can be used directly (Equations A7 and A11 of their work). After nondimensionalization, the part of the force due to the incoming difference-frequency second-order wave potential is:

$$
\bar{f}_{I, \phi^{(2)}, j l}^{-}=\pi \bar{\omega}_{j l}^{-}\left(\bar{\gamma}_{j l}^{-}+\bar{\gamma}_{l j}^{-*}\right) \frac{\tanh \left(\bar{k}_{j l}^{-\bar{h}}\right)}{\bar{k}_{j l}^{-} \bar{h}} J_{1}\left(\bar{k}_{j l}^{-}\right)
$$

with

$$
\bar{\gamma}_{j l}^{-}=-i \frac{\left(\bar{k}_{j} \bar{h}\right)^{2}\left[1-\tanh ^{2}\left(\bar{k}_{j} \bar{h}\right)\right]-2\left(\bar{k}_{j} \bar{h}\right)\left(\bar{k}_{l} \bar{h}\right)\left[1+\tanh \left(\bar{k}_{j} \bar{h}\right) \tanh \left(\bar{k}_{l} \bar{h}\right)\right]}{2 \sqrt{\bar{k}_{j} \bar{h} \tanh \left(\bar{k}_{j} \bar{h}\right)}\left[\bar{\omega}_{j l}^{-2}-\bar{k}_{j l}^{-} \bar{h} \tanh \left(\bar{k}_{j l}^{-\bar{h}}\right)\right]}
$$

while the contribution of its body perturbation counterpart is given by:

$$
\begin{array}{r}
\bar{f}_{P, \phi^{(2)}, j l}^{-}=-\pi \bar{\omega}_{j l}^{-}\left(\bar{\gamma}_{j l}^{-}+\bar{\gamma}_{l j}^{-*}\right) \frac{\bar{k}_{j l}^{-} \bar{h}}{\cosh \left(\bar{k}_{j l}^{-} \bar{h}\right)} J_{1}\left(\bar{k}_{j l}^{-}\right)\left[B_{0, j l} \Pi_{0, j l} \frac{H_{1}\left(v_{0, j l}\right)}{v_{0, j l} H_{1}^{\prime}\left(v_{0, j l}\right)}\right. \\
\left.+\sum_{n=1}^{\infty} B_{n, j l} \Pi_{n, j l} \frac{K_{1}\left(\kappa_{n, j l}\right)}{\kappa_{n, j l} K_{1}^{\prime}\left(\kappa_{n, j l}\right)}\right]
\end{array}
$$

where

$$
\begin{aligned}
& \Pi_{n, j l}=\frac{1}{2}\left[\frac{\sinh \left(\bar{k}_{j l}^{-} \bar{h}+v_{n, j l}\right)}{\bar{k}_{j l}^{-} \bar{h}+v_{n, j l}}+\frac{\sinh \left(\bar{k}_{j l}^{-} \bar{h}-v_{n, j l}\right)}{\bar{k}_{j l}^{-} \bar{h}-v_{n, j l}}\right] \\
& B_{n, j l}=\frac{4 \sinh \left(v_{n, j l}\right)}{2 v_{n, j l}+\sinh \left(2 v_{n, j l}\right)} \\
& v_{n, j l}= \begin{cases}\frac{\bar{\omega}_{j l}^{-2}}{\tanh v_{0, j l}} & \text { for } n=0 \\
i \kappa_{n, j l} & \text { for } n \neq 0\end{cases} \\
& \bar{\omega}_{j l}^{-2}=-\kappa_{n, j l} \tan \kappa_{n, j l}, \quad\left(n-\frac{1}{2}\right) \pi \leq \kappa_{n} \leq n \pi
\end{aligned}
$$

The expressions for the nondimensional moment components acting on the body, $\bar{m}_{j l}^{-}=m_{j l}^{-} /(\rho g R h)$, are very similar, as the difference is only in the integration along $Z$, which is quite simple. Expressing the moment with respect to $Z z=0$, the components due to the wave elevation, $\bar{m}_{I, \eta, j l}$ and $\bar{m}_{P, \eta, j l}$, are zero, while for the others it suffices to replace the expressions of $I_{j l}^{ \pm}$and $\Pi_{n, j l}$ by:

$$
\begin{aligned}
\hat{I}_{j l}^{ \pm} & =\frac{1}{2}\left[\frac{1-\cosh \left(\bar{k}_{j l}^{+} \bar{h}\right)}{\left(\bar{k}_{j l}^{+} \bar{h}\right)^{2}} \pm \frac{1-\cosh \left(\bar{k}_{j l}^{-} \bar{h}\right)}{\left(\bar{k}_{j l}^{-} \bar{h}\right)^{2}}\right] \\
\hat{\Pi}_{n, j l} & =\frac{1}{2}\left[\frac{1-\cosh \left(\bar{k}_{j l}^{-} \bar{h}+v_{n, j l}\right)}{\left(\bar{k}_{j l}^{-} \bar{h}+v_{n, j l}\right)^{2}}+\frac{1-\cosh \left(\bar{k}_{j l}^{-} \bar{h}-v_{n, j l}\right)}{\left(\bar{k}_{j l}^{-} \bar{h}-v_{n, j l}\right)^{2}}\right]
\end{aligned}
$$


APPENDIX D. Semi-analytic solution for the difference-frequency second-order wave forces on a

The mean forces on the bottom mounted cylinder, which are required by Newman's approximation, are a special case of the low-frequency forces given above. They can be found in Kim and Yue (1989), and result in the following after nondimensionalization:

$$
\begin{aligned}
& \bar{f}_{j j}^{-}=\frac{4}{\pi^{2} \bar{k}_{j}^{2}}\left(1+\frac{2 \bar{k}_{j} \bar{h}}{\sinh \left(2 \bar{k}_{j} \bar{h}\right)}\right) \sum_{n=0}^{\infty} \frac{\left[1-n(n+1) / \bar{k}_{j}^{2}\right]^{2}}{\left[J_{n}^{\prime 2}\left(\bar{k}_{j}\right)+Y_{n}^{\prime 2}\left(\bar{k}_{j}\right)\right]\left[J_{n+1}^{\prime 2}\left(\bar{k}_{j}\right)+Y_{n+1}^{\prime 2}\left(\bar{k}_{j}\right)\right]} \\
& \bar{m}_{j j}^{-}=-\bar{f}_{j j}^{-}+\Re\left\{\frac{-4 i}{\pi \bar{k}_{j}^{2}} \sum_{n=0}^{\infty} \frac{1}{H_{n}^{\prime}\left(\bar{k}_{j}\right) H_{n+1}^{\prime *}\left(\bar{k}_{j}\right)}\right. \\
& \left.\cdot\left[-1+\frac{2 \bar{k} \bar{h}}{\sinh \left(2 \bar{k}_{j} \bar{h}\right)}\left(\left(\frac{n(n+1)}{\bar{k}_{j}^{2}}+1\right) \mathcal{Z}\left(\bar{k}_{j}\right)-\frac{1}{2}\right)\right]\right\}
\end{aligned}
$$

with

$$
\mathcal{Z}\left(\bar{k}_{j}\right)=\frac{1}{4}+\frac{2 \bar{k}_{j} \bar{h} \sinh \left(2 \bar{k}_{j} \bar{h}\right)-\cosh \left(2 \bar{k}_{j} \bar{h}\right)+1}{8\left(\bar{k}_{j} \bar{h}\right)^{2}}
$$




\section{APPENDIX E - Methodology to post process the forced oscillation tests}

The result of each run of the forced oscillation tests is a time series of the force exerted by the actuator, which is related to the imposed acceleration by:

$$
M \ddot{q}=F_{\text {hydro, q }}+F_{\text {actuator, q }}+F_{\text {noise, q }}
$$

with $q$ the motion along the axis of the actuator, known to be sinusoidal with amplitude $A$ and frequency $\omega=2 \pi / T$ :

$$
q=A \sin (\omega t)
$$

and $M$ the total mass of the assembly, i.e. the hull model, the load cells and elements that were used to attach the model to the actuator. The force $F_{\text {noise }}$ accounts for the highfrequency noise that is present in the measured force, most notably due to the vibration of the instrumentation bridge to which the equipment is attached. For the horizontal oscillations, $q=x$ and $M=13.848 \mathrm{~kg}$ (model scale), while $q=z$ and $M=15.285 \mathrm{~kg}$ for the vertical oscillations due to changes in the setup used to keep the model at place. The model was placed in such a way that it was in hydrostatic equilibrium, and this is why the buoyancy force and weight are not included in the equation above.

The hydrodynamic force, $F_{\text {hydro }}$, is assumed to be composed of Morison's equation, a potential damping term and a hydrostatic restoring force:

$$
F_{\text {hydro, } q}=-\frac{1}{2} \rho C_{D q}^{\prime} S_{q}|\dot{q}| \dot{q}-\rho C_{a q}^{\prime} \mathcal{V}_{q} \ddot{q}-B_{q}^{\text {pot }} \dot{q}-K_{q} q
$$

where $K_{q}$ the hydrostatic stiffness, with $K_{x}=0$ and $K_{z}=\rho g S_{z}$. The equations can be rearranged as follows:

$$
F_{\text {morison, q }}=\frac{1}{2} \rho C_{D q}^{\prime} S_{q}|\dot{q}| \dot{q}+\rho C_{a q}^{\prime} V_{q} \ddot{q}=F_{\text {actuator, } \mathrm{q}}+F_{\text {noise, } \mathrm{q}}-M \ddot{q}-B_{\text {pot }} \dot{q}-K_{q} q
$$

As the body is moving according to a prescribed motion, the position and body acceleration are known, hence the terms $M \ddot{q}$ and $K_{q} q$ can be easily removed from the measured signal $F_{\text {actuator, q }}$. The potential damping term, however, requires $B_{\text {pot }}$ to be evaluated somehow. As this force component is well modeled by potential codes, the results obtained with WAMIT, given in Figure 65, are used to compute this term. Note that the potential damping is very small for large periods of motion, resulting in a negligible contribution to the total force in the largest period of oscillation that highlights the importance of viscous damping for the slow surge motion. Details about the WAMIT model are given in Section 6.3.1. 
Figure 65 - Nondimensional potential damping in surge $\left(B_{11}\right)$ and heave $\left(B_{33}\right)$ calculated with WAMIT.

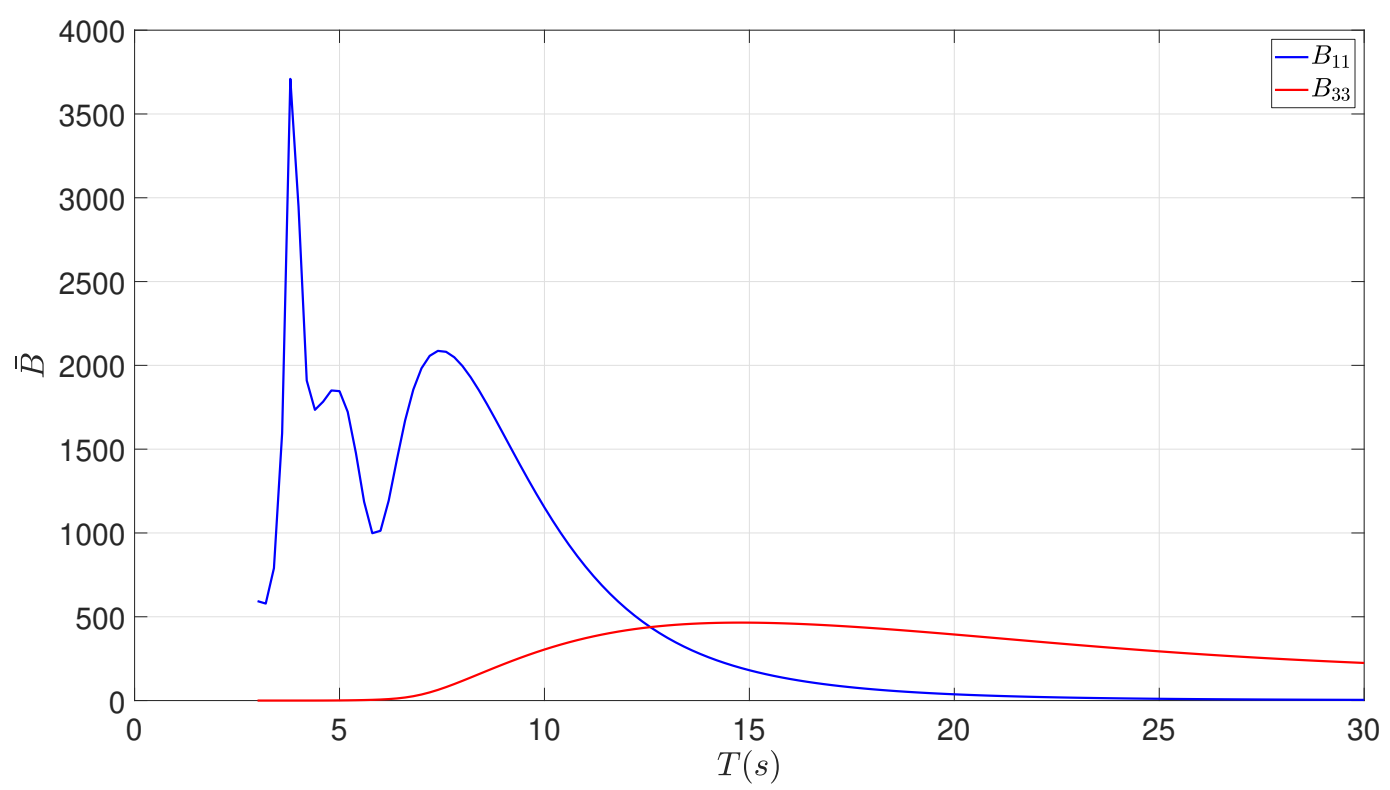

The noise is removed by filtering the signal using a simple Fast Fourier Transform (FFT) filter, i.e. the filtered signal is obtained by computing the discrete Fourier transform of the measured signal, removing the frequencies above a threshold and then reconverting it back to time domain. This threshold was established empirically, and a value of $0.22 \mathrm{~Hz}$ for the lowest period $(T=12 \mathrm{~s})$ and one of $0.06 \mathrm{~Hz}$ for the largest period ( $T=84.4 \mathrm{~s})$ were found to provide good results.

Two examples of the signal before and after the filtering process are presented in Figures 66 and 67 . Both refer to the horizontal oscillations, but the first one corresponds to the lowest oscillation period and largest motion amplitude, $T=12 \mathrm{~s}$ and $A=4.8 \mathrm{~m}$, while the second one corresponds to the largest period and lowest motion amplitude, $T=84.4 \mathrm{~s}$ and $A=0.8 \mathrm{~m}$. As the force of interest, $F_{\text {morison, }}$ is proportional to the body velocity and acceleration, it becomes increasingly small as the motion amplitude decreases or as the period of oscillation increases, in such a way that the high-frequency noise can be significantly larger than $F_{\text {morison }}$ for the tests involving slow motions. Nevertheless, Figure 67 shows that the forces around the motion frequency, in this case $0.012 \mathrm{~Hz}$, are well represented even when they can not be observed in the corresponding time series.

Figures 66 and 67 also present the force calculated with Morison's equation using the resulting coefficients $C_{a}$ and $C_{D}$. These are obtained from $F_{\text {morison }}$ following the procedure detailed by Keulegan and Carpenter (1958) and reproduced below. The first step is to expand the force $F_{\text {morison }}$ as a Fourier series:

$$
\frac{F_{\text {morison }}}{\rho S_{q} \omega^{2} A^{2}}=\sum_{n=0}^{\infty} A_{n} \sin (n \omega t)+B_{n} \cos (n \omega t)
$$


Figure 66 - Time series (top) and discrete fourier transform (bottom) of the hydrodynamic force in surge direction measured in the experiments before and after the filtering process for $T=12 \mathrm{~s}$ and $A=4.8 \mathrm{~m}$. The force calculated with Morison's equation considering the resulting $C_{a}$ and $C_{D}$ is also plotted.
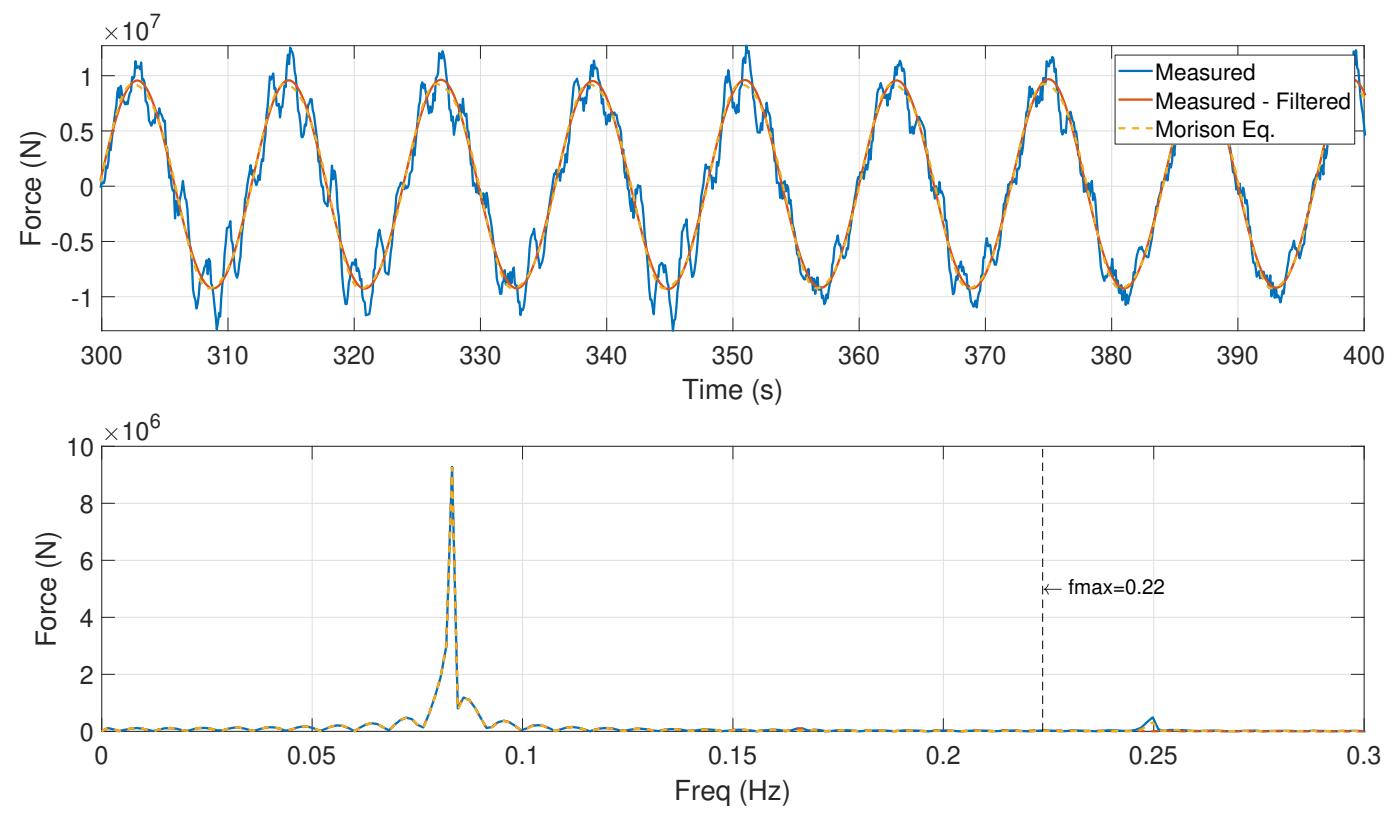

Figure 67 - Time series (top) and discrete fourier transform (bottom) of the hydrodynamic force in surge direction measured in the experiments before and after the filtering process for $T=84.4 \mathrm{~s}$ and $A=0.8 \mathrm{~m}$. The force calculated with Morison's equation considering the resulting $C_{a}$ and $C_{D}$ is also plotted.
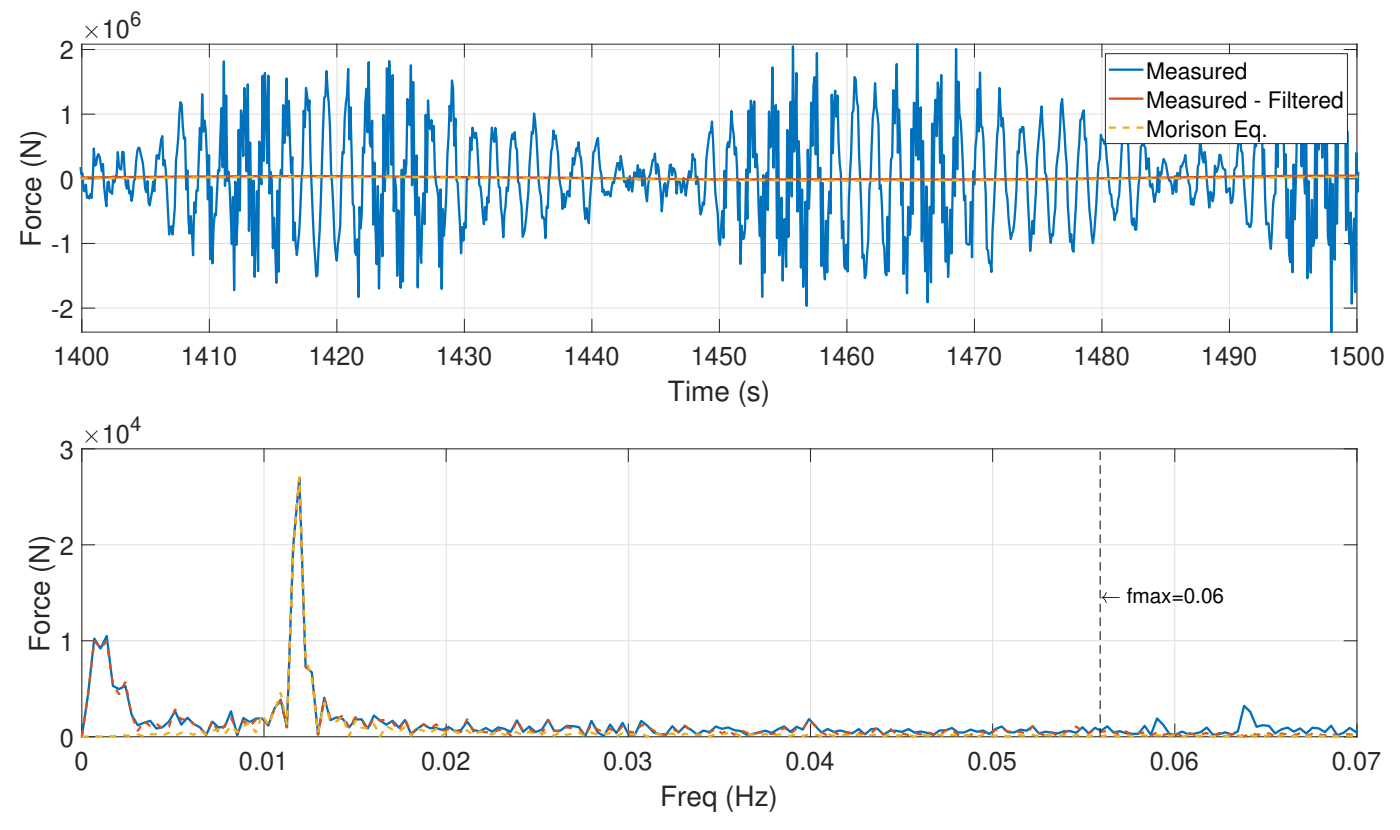

with

$$
A_{n}=\frac{1}{\pi} \int_{0}^{2 \pi} F_{\text {morison }} \sin (n \omega t) \mathrm{d} t
$$




$$
B_{n}=\frac{1}{\pi} \int_{0}^{2 \pi} F_{\text {morison }} \cos (n \omega t) \mathrm{d} t
$$

as a consequence of the symmetry of the force with respect to time, i.e. $F(\omega t)=-F(\omega t+\pi)$, the coefficients are equal to zero when $n$ is even. The objective is to compare this expression with Equation E.4, which can be rewritten using Equation E.2 as follows:

$$
\frac{F_{\text {morison }}}{\rho S_{q} \omega^{2} A^{2}}=\frac{1}{2} C_{D q}^{\prime}|\cos (\omega t)| \cos (\omega t)-C_{a q}^{\prime} \frac{V_{q}}{A S_{q}} \sin (\omega t)
$$

In order to compare Equations E.5 and E.8, a term with $|\cos (\omega t)| \cos (\omega t)$ is introduced by using the Fourier series of this function as well:

$$
|\cos (\omega t)| \cos (\omega t)=\sum b_{n} \cos (n \omega t)
$$

where only the coefficients multiplying cosines are kept due to $|\cos (\omega t)| \cos (\omega t)$ being an even function. The coefficients $b_{n}$ are given by:

$$
b_{n}=\frac{1}{\pi} \int_{0}^{2 \pi}|\cos (\omega t)| \cos (\omega t) \cos (n \omega t) \mathrm{d} t= \begin{cases}0 & \text { for } n \text { even } \\ (-1)^{\frac{n+1}{2}} \frac{8}{n\left(n^{2}-4\right) \pi} & \text { for } n \text { odd }\end{cases}
$$

Isolating the term with $\cos (\omega t)$ from the others yields:

$$
\cos (\omega t)=\frac{|\cos (\omega t)| \cos (\omega t)}{b_{1}}-\frac{1}{b_{1}}\left[b_{3} \cos (3 \omega t)+a_{5} \cos (5 \omega t)+\ldots\right]
$$

which can now be used to replace the cosine term from Equation E.5, made easier by introducing the following coefficients:

$$
\begin{aligned}
& B_{1}^{\prime}=\frac{B_{1}}{b_{1}} \\
& B_{3}^{\prime}=B_{3}-\frac{b_{3}}{b_{1}} B_{1} \\
& B_{5}^{\prime}=B_{5}-\frac{b_{5}}{b_{1}} B_{1}
\end{aligned}
$$

yielding

$$
\begin{aligned}
\frac{F_{\text {morison }}}{\rho S_{q} \omega^{2} A^{2}} & =A_{1} \sin (\omega t)+A_{3} \sin (3 \omega t)+A_{5} \sin (5 \omega t)+\ldots \\
& +B_{1}^{\prime}|\cos (\omega t)| \cos (\omega t)+B_{3}^{\prime} \cos (3 \omega t)+B_{5}^{\prime} \sin (5 \omega t)+\ldots
\end{aligned}
$$

Following Keulegan and Carpenter (1958), Equation E.13 is compared with Equation E.8, leading to two time varying coefficients $C_{a q}^{\prime}(t)$ and $C_{D q}^{\prime}(t)$. After discussing different ways of dealing with this issue in their work, the chosen alternative is to state 
Morison's equation in terms of fixed coefficients $C_{a q}$ and $C_{D q}$ plus a remainder term, $\Delta R$, that includes all higher harmonics:

$$
\frac{F_{\text {morison }}}{\rho S_{q} \omega^{2} A^{2}}=\frac{1}{2} C_{D q}|\cos (\omega t)| \cos (\omega t)-C_{a q} \frac{V_{q}}{A S_{q}} \sin (\omega t)+\Delta R
$$

with the different terms obtained by direct comparison with Equation E.13:

$$
\begin{aligned}
C_{D q} & =2 B_{1}^{\prime} \\
C_{a q} & =-\frac{A S_{q}}{V_{q}} A_{1} \\
\Delta R & =A_{3} \sin (3 \omega t)+A_{5} \sin (5 \omega t)+\ldots \\
& +B_{3}^{\prime} \cos (3 \omega t)+B_{5}^{\prime} \cos (5 \omega t)+\ldots
\end{aligned}
$$

Hence, in a nutshell, the procedure of obtaining the added mass and drag coefficients from the forced oscillations adopted in the present work consists of: 1) preprocessing the measured signal to remove noise and additional force components (namely inertia, potential damping and hydrostatic restoration); 2) evaluating the Fourier coefficients from the resulting signal; and 3) calculating $C_{D q}$ and $C_{a q}$ with Equations E.15 and E.16. 